ANALYSIS \& PDE Volume $6 \quad$ No. $6 \quad 2013$

DAVID CHIRON

STABUITY AND INSTABILITY FOR SUBSONIC TRAVELING WAVES

OF THE NONLINEAR SCHRÖDINGER EQUATION IN DIMENSION ONE: 


\title{
STABILITY AND INSTABILITY FOR SUBSONIC TRAVELING WAVES OF THE NONLINEAR SCHRÖDINGER EQUATION IN DIMENSION ONE
}

\author{
DAVID CHIRON
}

\begin{abstract}
We study the stability/instability of the subsonic traveling waves of the nonlinear Schrödinger equation in dimension one. Our aim is to propose several methods for showing instability (use of the GrillakisShatah-Strauss theory, proof of existence of an unstable eigenvalue via an Evans function) or stability. For the latter, we show how to construct in a systematic way a Liapounov functional for which the traveling wave is a local minimizer. These approaches allow us to give a complete stability/instability analysis in the energy space including the critical case of the kink solution. We also treat the case of a cusp in the energy-momentum diagram.
\end{abstract}

\section{Introduction}

This paper is a continuation of our previous work [Chiron 2012], where we consider the one-dimensional nonlinear Schrödinger equation

$$
i \frac{\partial \Psi}{\partial t}+\partial_{x}^{2} \Psi+\Psi f\left(|\Psi|^{2}\right)=0
$$

This equation appears as a relevant model in condensed matter physics: Bose-Einstein condensation and superfluidity (see [Roberts and Berloff 2001; Ginzburg and Pitaevskiǔ 1958; Gross 1963; Abid et al. 2003]); nonlinear optics (see, for instance, the survey [Kivshar and Luther-Davies 1998]). Several nonlinearities may be encountered in physical situations: $f(\varrho)= \pm \varrho$ gives rise to the focusing/defocusing cubic NLS; $f(\varrho)=1-\varrho$ to the so-called Gross-Pitaevskii equation; $f(\varrho)=-\varrho^{2}$ (see [Kolomeisky et al. 2000] for Bose-Einstein condensates); more generally a pure power; the "cubic-quintic" NLS (see [Barashenkov and Panova 1993]), where

$$
f(\varrho)=-\alpha_{1}+\alpha_{3} \varrho-\alpha_{5} \varrho^{2}
$$

and $\alpha_{1}, \alpha_{3}$ and $\alpha_{5}$ are positive constants such that $f$ has two positive roots; and in nonlinear optics, we may take (see [Kivshar and Luther-Davies 1998])

$$
f(\varrho)=-\alpha \varrho^{v}-\beta \varrho^{2 v}, \quad f(\varrho)=-\frac{\varrho_{0}}{2}\left(\frac{1}{\left(1+\frac{1}{\varrho_{0}}\right)^{v}}-\frac{1}{\left(1+\frac{\varrho}{\varrho_{0}}\right)^{v}}\right), \quad f(\varrho)=-\alpha \varrho\left(1+\gamma \tanh \frac{\varrho^{2}-\varrho_{0}^{2}}{\sigma^{2}}\right)
$$

MSC2010: 35B35, 35J20, 35Q40, 35Q55, 35C07.

Keywords: traveling wave, nonlinear Schrödinger equation, Gross-Pitaevskii equation, stability, Evans function, Liapounov functional. 
where $\alpha, \beta, \gamma, \nu, \sigma>0$ are given constants (the second one, for instance, takes into account saturation effects), etc. As a consequence, as in [Chiron 2012], we shall consider a rather general nonlinearity $f$, with $f$ of class $\mathscr{C}^{2}$. In the context of Bose-Einstein condensation or nonlinear optics, the natural condition at infinity appears to be

$$
|\Psi|^{2} \rightarrow r_{0}^{2} \quad \text { as }|x| \rightarrow+\infty
$$

where $r_{0}>0$ is such that $f\left(r_{0}^{2}\right)=0$.

For solutions $\Psi$ of (NLS) which do not vanish, we may use the Madelung transform

$$
\Psi=A \exp (i \phi)
$$

and rewrite (NLS) as an hydrodynamical system with an additional quantum pressure

$$
\left\{\begin{array} { l } 
{ \partial _ { t } A + 2 \partial _ { x } \phi \partial _ { x } A + A \partial _ { x } ^ { 2 } \phi = 0 , } \\
{ \partial _ { t } \phi + ( \partial _ { x } \phi ) ^ { 2 } - f ( A ^ { 2 } ) - \frac { \partial _ { x } ^ { 2 } A } { A } = 0 }
\end{array} \text { or } \quad \left\{\begin{array}{l}
\partial_{t} \rho+2 \partial_{x}(\rho u)=0, \\
\partial_{t} u+2 u \partial_{x} u-\partial_{x}(f(\rho))-\partial_{x}\left(\frac{\partial_{x}^{2}(\sqrt{\rho})}{\sqrt{\rho}}\right)=0,
\end{array}\right.\right.
$$

with $(\rho, u) \equiv\left(A^{2}, \partial_{x} \phi\right)$. When neglecting the quantum pressure and linearizing this Euler system around the particular trivial solution $\Psi=r_{0}$ (or $(A, u)=\left(r_{0}, 0\right)$ ), we obtain the free wave equation

$$
\left\{\begin{array}{l}
\partial_{t} \bar{A}+r_{0} \partial_{x} \bar{U}=0 \\
\partial_{t} \bar{U}-2 r_{0} f^{\prime}\left(r_{0}^{2}\right) \partial_{x} \bar{A}=0,
\end{array}\right.
$$

with associated speed of sound

$$
\mathfrak{c}_{s} \equiv \sqrt{-2 r_{0}^{2} f^{\prime}\left(r_{0}^{2}\right)}>0,
$$

provided $f$ satisfies the defocusing assumption $f^{\prime}\left(r_{0}^{2}\right)<0$ (that is, the Euler system is hyperbolic in the region $\rho \simeq r_{0}^{2}$ ), which we will assume throughout the paper. Concerning the rigorous justification of the free wave regime for the Gross-Pitaevskii equation (in arbitrary dimension), see [Béthuel et al. 2010]. The speed of sound $\mathfrak{c}_{s}$ enters in a crucial way in the question of existence of traveling waves for (NLS) with modulus tending to $r_{0}$ at infinity (see, e.g., [Chiron 2012]).

The nonlinear Schrödinger equation formally preserves the energy

$$
E(\psi) \equiv \int_{\mathbb{R}}\left|\partial_{x} \psi\right|^{2}+F\left(|\psi|^{2}\right) d x,
$$

where $F(\varrho) \equiv \int_{\varrho}^{r_{0}^{2}} f$. Since

$$
F(\varrho) \sim \frac{\mathfrak{c}_{s}^{2}}{8 r_{0}^{2}}\left(\varrho-r_{0}^{2}\right)^{2} \sim \frac{\mathfrak{c}_{s}^{2}}{2}\left(\sqrt{\varrho}-r_{0}\right)^{2}
$$

when $\varrho \rightarrow r_{0}^{2}$, it follows that the natural energy space turns out to be the space

$$
\mathscr{L} \equiv\left\{\psi \in L^{\infty}(\mathbb{R}), \partial_{x} \psi \in L^{2}(\mathbb{R}),|\psi|-r_{0} \in L^{2}(\mathbb{R})\right\} \subset \mathscr{C}_{b}(\mathbb{R}, \mathbb{C}),
$$

endowed with the distance

$$
d_{\mathscr{E}}(\psi, \tilde{\psi}) \equiv\left\|\partial_{x} \psi-\partial_{x} \tilde{\psi}\right\|_{L^{2}(\mathbb{R})}+\||\psi|-|\tilde{\psi}|\|_{L^{2}(\mathbb{R})}+|\psi(0)-\tilde{\psi}(0)| .
$$


The Cauchy problem was shown to be locally well posed in the Zhidkov space $\left\{\psi \in L^{\infty}(\mathbb{R}), \partial_{x} \psi \in L^{2}(\mathbb{R})\right\}$ by P. Zhidkov [2001] (see also the work by C. Gallo [2004]). For global well-posedness results, see [Gallo 2008; Gérard 2008]. More precisely, the local well-posedness we shall use is the following.

Theorem 1 [Zhidkov 2001; Gallo 2004]. Let $\Psi^{\text {in }} \in \mathscr{L}$. Then, there exists $T_{*}>0$ and a unique solution $\Psi$ to (NLS) such that $\Psi_{\mid t=0}=\Psi^{\text {in }}$ and $\Psi-\Psi^{\text {in }} \in \mathscr{C}\left(\left[0, T_{*}\right), H^{1}(\mathbb{R})\right)$. Moreover, $E(\Psi(t))$ does not depend on $t$.

The other quantity formally conserved by the Schrödinger flow, due to the invariance by translation, is the momentum. The momentum is not easy to define in dimension one for maps that vanish somewhere (see [Béthuel et al. 2008a; 2008b]). However, if $\psi$ does not vanish, we have a lifting $\psi=A \mathrm{e}^{i \phi}$, and then the correct definition of the momentum is given by [Kivshar and Yang 1994]

$$
P(\psi) \equiv \int_{\mathbb{R}}\left\langle i \psi \mid \partial_{x} \psi\right\rangle\left(1-\frac{r_{0}^{2}}{|\psi|^{2}}\right) d x=\int_{\mathbb{R}}\left(A^{2}-r_{0}^{2}\right) \partial_{x} \phi d x,
$$

where $\langle\cdot \mid \cdot\rangle$ denotes the real scalar product in $\mathbb{C}$. We define

$$
\mathscr{L}_{\text {hy }} \equiv\left\{v \in \mathscr{L}, \inf _{\mathbb{R}}|v|>0\right\},
$$

which is the open subset of $\mathscr{E}$ in which we have lifting and where the hydrodynamical formulation (2) of (NLS) is possible through the Madelung transform. It turns out that, if the initial datum belongs to $\mathscr{L}_{\text {hy }}$, the solution of (NLS) provided by Theorem 1 remains in $\mathscr{L}_{\text {hy }}$ for small times, and that the momentum is indeed conserved on this time interval (see [Gallo 2004]).

1A. The traveling waves and energy-momentum diagrams. The traveling waves with speed of propagation $c$ are special solutions of (NLS) of the form

$$
\Psi(t, x)=U(x-c t) .
$$

The profile $U$ has then to solve the ODE

$$
\partial_{x}^{2} U+U f\left(|U|^{2}\right)=i c \partial_{x} U
$$

together with the condition $|U(x)| \rightarrow r_{0}$ as $x \rightarrow \pm \infty$. These particular solutions play an important role in the long-time dynamics of (NLS) with nonzero condition at infinity. Possibly conjugating ( $\mathrm{TW}_{c}$ ), we see that we may assume that $c \geq 0$ without loss of generality. Moreover, we shall restrict ourselves to traveling waves which belong to the energy space $\mathscr{L}$ (so that $|U| \rightarrow r_{0}$ at $\pm \infty$ by the Sobolev embedding $\left.H^{1}(\mathbb{R}) \hookrightarrow \mathscr{C}_{0}(\mathbb{R}, \mathbb{C}) \equiv\left\{h \in \mathscr{C}(\mathbb{R}, \mathbb{C}), \lim _{ \pm \infty} h=0\right\}\right)$. For traveling waves $U_{c} \in \mathscr{L}$ that do not vanish in $\mathbb{R}$, hence that may be lifted to $U_{c}=A_{c} \mathrm{e}^{i \phi_{c}}$, the ODE $\left(\mathrm{TW}_{c}\right.$ ) can be transformed (see, e.g., [Chiron 2012]) into the system

$$
\partial_{x} \phi_{c}=\frac{c}{2} \cdot \frac{\eta_{c}}{\eta_{c}+r_{0}^{2}}, \quad 2 \partial_{x}^{2} \eta_{c}+\mathscr{V}_{c}^{\prime}\left(\eta_{c}\right)=0, \quad \text { with } \eta_{c} \equiv A_{c}^{2}-r_{0}^{2},
$$

and where the function $\mathscr{V}_{c}$ is related to $f$ by the formula

$$
\mathscr{V}_{c}(\xi) \equiv c^{2} \xi^{2}-4\left(r_{0}^{2}+\xi\right) F\left(r_{0}^{2}+\xi\right) .
$$


To a nontrivial traveling wave $U_{c}$ is associated (see [ibid.]) some $\xi_{c} \geq-r_{0}^{2}$ such that $\mathscr{V}_{c}\left(\xi_{c}\right)=0 \neq \mathscr{V}_{c}^{\prime}\left(\xi_{c}\right)$ and $\mathscr{V}_{c}$ is negative between $\xi_{c}$ and $-r_{0}^{2}$, and $\eta_{c}$ varies between 0 and $\xi_{c}$; that is, $\left\{\inf _{\mathbb{R}}\left|U_{c}\right|, \sup _{\mathbb{R}}\left|U_{c}\right|\right\}=$ $\left\{r_{0}, \sqrt{r_{0}^{2}+\xi_{c}}\right\}$. Moreover, the only traveling wave solution (if it exists) that vanishes somewhere is for $c=0$ and is called the kink: it is an odd solution (up to a space translation) and then $\xi_{0}=0$.

We have also seen in [ibid.] that any traveling wave in $\mathscr{E}$ with speed $c>\mathfrak{c}_{s}$ is constant, and also that any nonconstant traveling wave in $\mathscr{L}$ of speed $c_{*} \in\left(0, \mathfrak{c}_{s}\right)$ belongs to a unique (up to the natural invariances: phase factor and translation) local branch $c \mapsto U_{c}$ defined for $c$ close to $c_{*}$.

In [ibid.], we have investigated the qualitative behaviors of the traveling waves for (NLS) with nonzero condition at infinity for a general nonlinearity $f$. A particular attention has been payed in [ibid.] to the transonic limit, where we have an asymptotic behavior governed by the Korteweg-de Vries or the generalized Korteweg-de Vries equation. In order to illustrate the very different situations we may encounter when we allow a general nonlinearity $f$, we give now some energy-momentum diagrams we have obtained (one is taken from the appendix in [Chiron and Scheid 2012], where we have performed numerical simulations in dimension two for the model cases we have studied in [Chiron 2012]):

- The Gross-Pitaevskii nonlinearity: $f(\varrho)=1-\varrho$ (see Figure 1).

- A cubic-quintic-septic nonlinearity: $f(\varrho)=-(\varrho-1)+\frac{3}{2}(\varrho-1)^{2}-\frac{3}{2}(\varrho-1)^{3}$ (see Figure 2).

- A cubic-quintic-septic nonlinearity: $f(\varrho) \equiv-4(\varrho-1)-36(\varrho-1)^{3}$ or $f(\varrho) \equiv-4(\varrho-1)-60(\varrho-1)^{3}$. For these two nonlinearities, the graph of $E$ and $P$ vs. speed $c$ is given in Figure 3, but the $(E, P)$ diagrams are, respectively, those in Figure 4.

- A cubic-quintic-septic nonlinearity: $f(\varrho) \equiv-\frac{1}{2}(\varrho-1)+\frac{3}{4}(\varrho-1)^{2}-2(\varrho-1)^{3}$ (see Figure 5).

- A degenerate case: $f(\varrho) \equiv-2(\varrho-1)+3(\varrho-1)^{2}-4(\varrho-1)^{3}+5(\varrho-1)^{4}-6(\varrho-1)^{5}$ (see Figure 6).

- A perturbation of the previous degenerate case: $f(\varrho) \equiv-2(\varrho-1)+\left(3-10^{-3}\right)(\varrho-1)^{2}-4(\varrho-1)^{3}+$ $5(\varrho-1)^{4}-6(\varrho-1)^{5}$ (see Figure 7).

- A saturated NLS: $f(\varrho) \equiv \exp \left((1-\varrho) / \varrho_{0}\right)-1$ with $\varrho_{0}=0.4$ (see Figure 8).

- Another saturated NLS: $f(\varrho) \equiv \frac{1}{2} \varrho_{0}\left(1 /\left(1+\varrho / \varrho_{0}\right)^{2}-1 /\left(1+1 / \varrho_{0}\right)^{2}\right)$, with $\varrho_{0}=0.08$ (see Figure 9).

- The cubic-quintic nonlinearity: $f(\varrho) \equiv-(\varrho-1)-3(\varrho-1)^{2}$ (see Figure 10).

Through the study (in [Chiron 2012]) of these model cases, we have shown that, if the energy-momentum diagram is well-known for the Gross-Pitaevskii equation, the qualitative properties of the traveling wave solutions can not be easily deduced from the global shape of the nonlinearity $f$. In particular, even if we restrict ourselves to smooth and decreasing nonlinearities (as is the Gross-Pitaevskii one), we see that we may have a great variety of behaviors: multiplicity of solutions, branches with diverging energy and momentum, nonexistence of traveling waves for some $c_{0} \in\left(0, \mathfrak{c}_{s}\right)$, branches of solutions that cross, existence of sonic traveling waves, transonic limit governed by the $\mathrm{mKdV}$ or more generally by the gKdV solitary wave equation instead of the usual $\mathrm{KdV}$ one, existence of cusps, etc. 

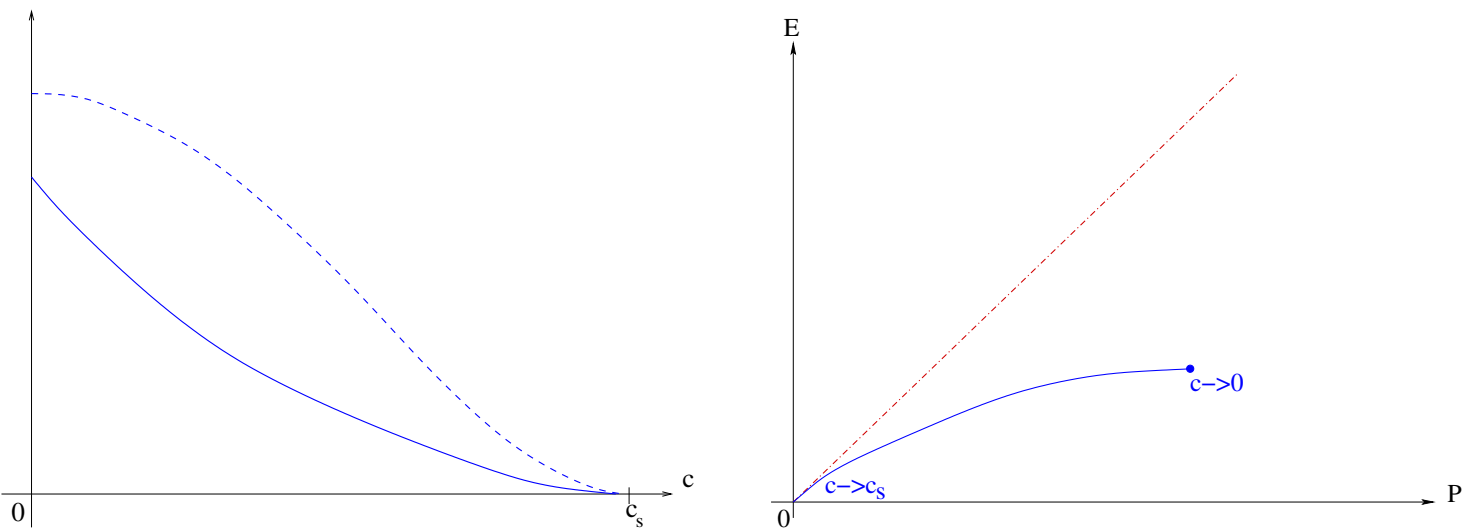

Figure 1. (a) Energy (dashed curve) and momentum (full curve) vs. speed; (b) $(E, P)$ diagram.
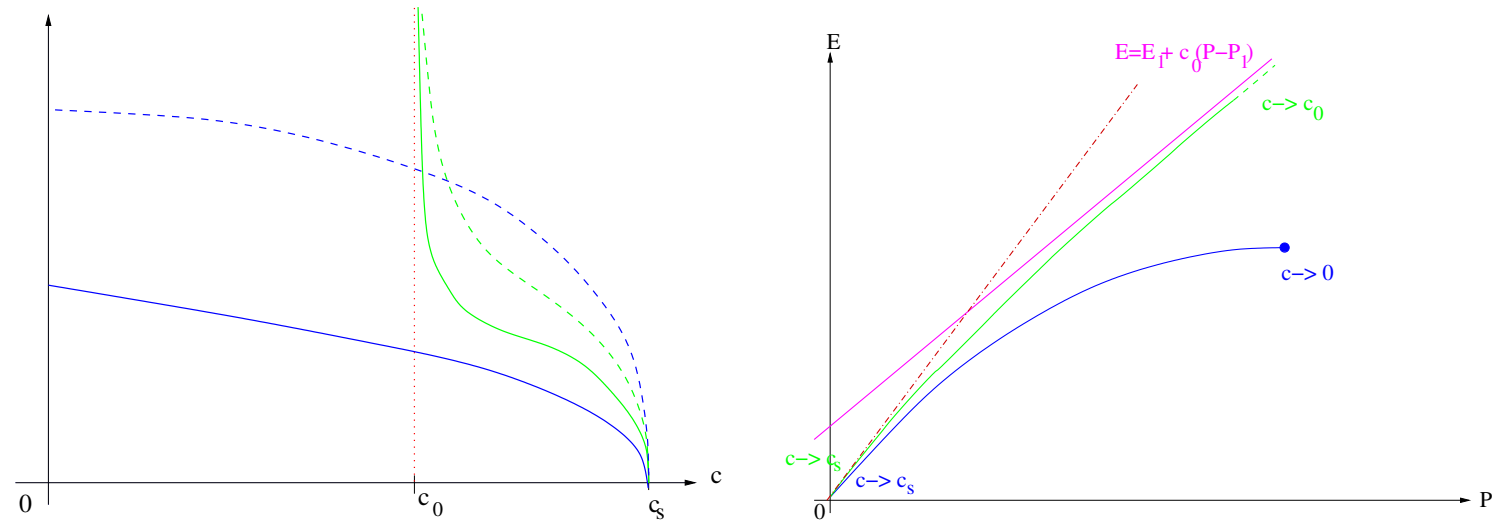

Figure 2. (a) Energy (dashed curve) and momentum (full curve) vs. speed; (b) $(E, P)$ diagram.

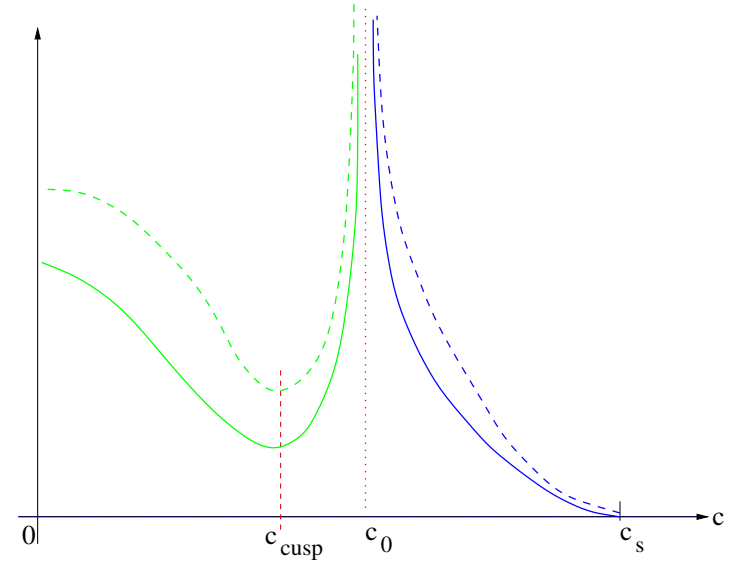

Figure 3. Energy (dashed curve) and momentum (full curve) vs. speed. 


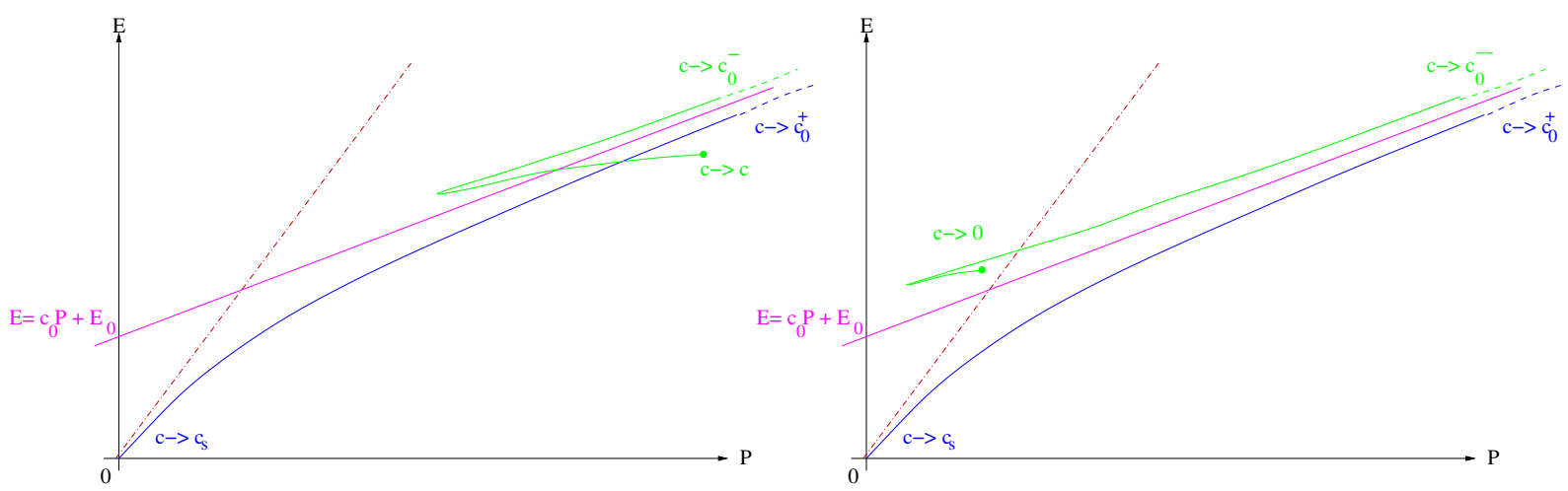

Figure 4. The two $(E, P)$ diagrams.
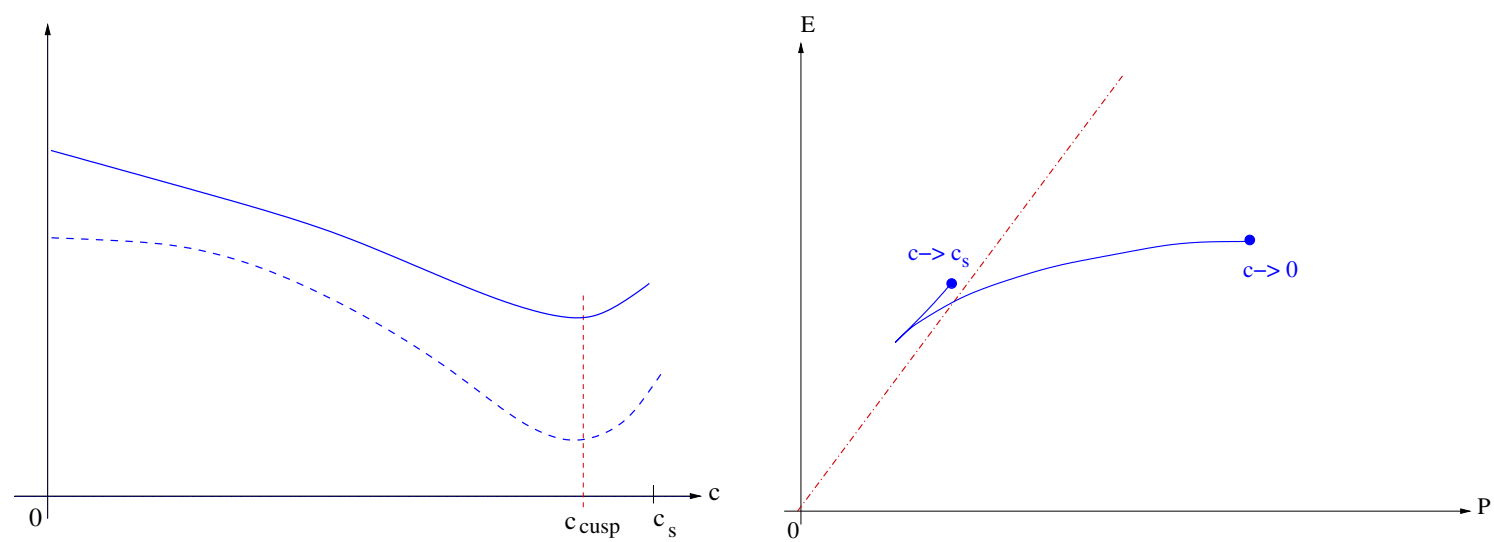

Figure 5. (a) Energy (dashed curve) and momentum (full curve) vs. speed; (b) $(E, P)$ diagram.
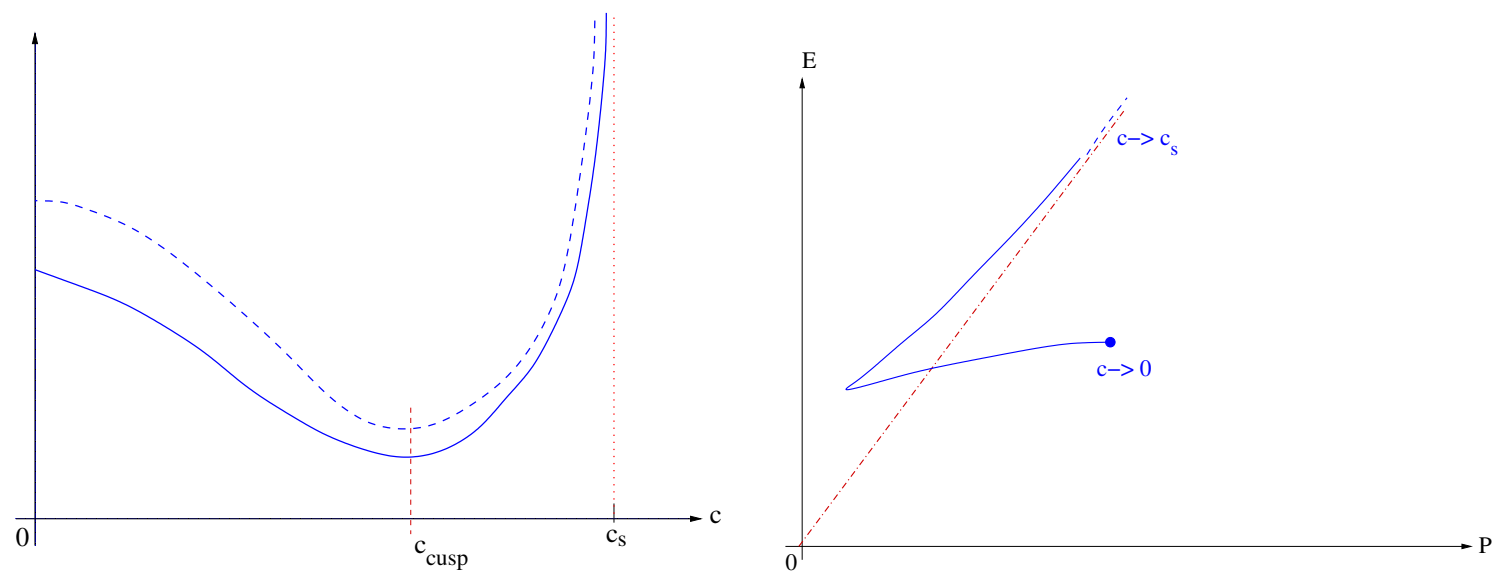

Figure 6. (a) Energy (dashed curve) and momentum (full curve) vs. speed; (b) $(E, P)$ diagram. 

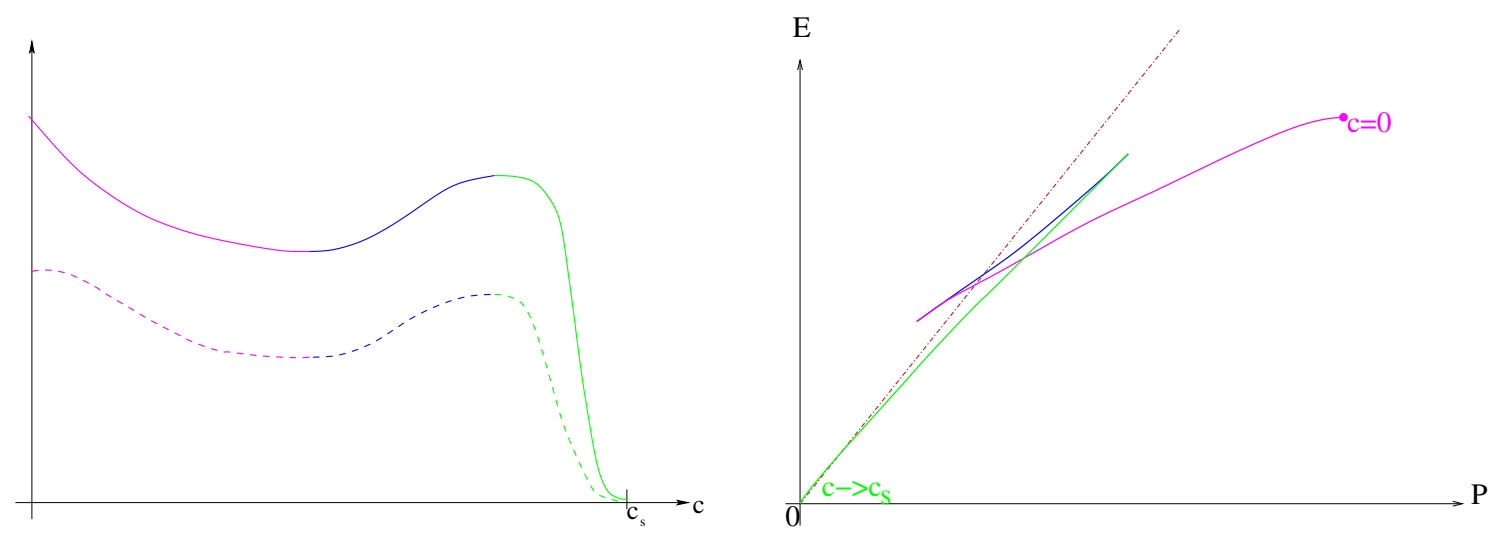

Figure 7. (a) Energy (dashed curve) and momentum (full curve) vs. speed; (b) qualitative $(E, P)$ diagram.
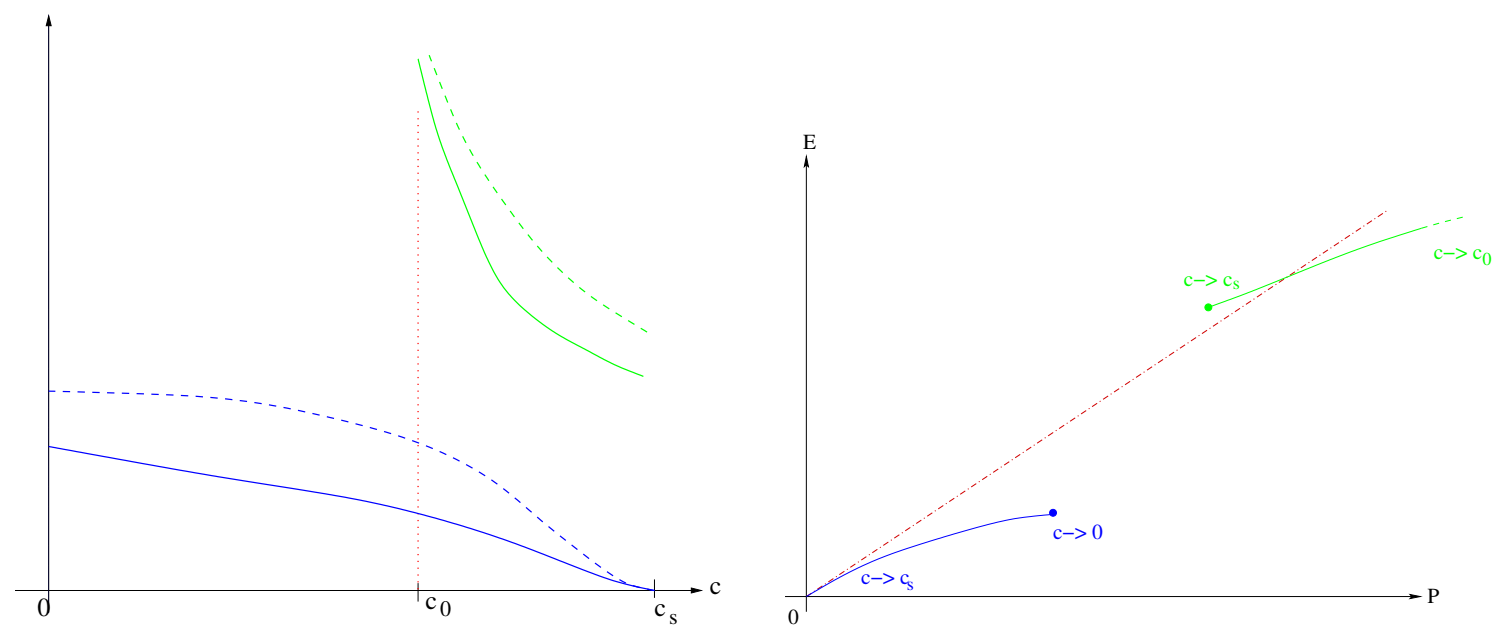

Figure 8. (a) Energy (dashed curve) and momentum (full curve) vs. speed; (b) $(E, P)$ diagram.

We investigate now the behavior at infinity of the nontrivial traveling waves, which depends on whether $c=\mathfrak{c}_{s}$ or not. We denote by $\mathbb{N}$ the set of nonnegative integers and $\mathbb{N}^{*}$ the set of positive integers. We consider for $m \in \mathbb{N}$ the following assumption:

$\left(A_{m}\right) f$ is of class $\mathscr{C}^{m+3}$ near $r_{0}^{2}$. Moreover, for $1 \leq j<m+2$, we have

$$
\frac{f^{(j)}\left(r_{0}^{2}\right)}{(j+1) !} r_{0}^{2 j}=(-1)^{j+1} \frac{\mathfrak{c}_{s}^{2}}{4} \quad \text { but } \quad \frac{f^{(m+2)}\left(r_{0}^{2}\right)}{(m+3) !} r_{0}^{2(m+2)} \neq(-1)^{m+3} \frac{\mathfrak{c}_{s}^{2}}{4}
$$

(note that, for $j=1$, equality always holds by definition of the speed of sound $\mathfrak{c}_{s}=\sqrt{-2 r_{0}^{2} f^{\prime}\left(r_{0}^{2}\right)}$ ).

Proposition 2. Let $U_{c} \in \mathscr{L}$ be a nonconstant traveling wave of speed $0 \leq c \leq \mathfrak{c}_{s}$. 

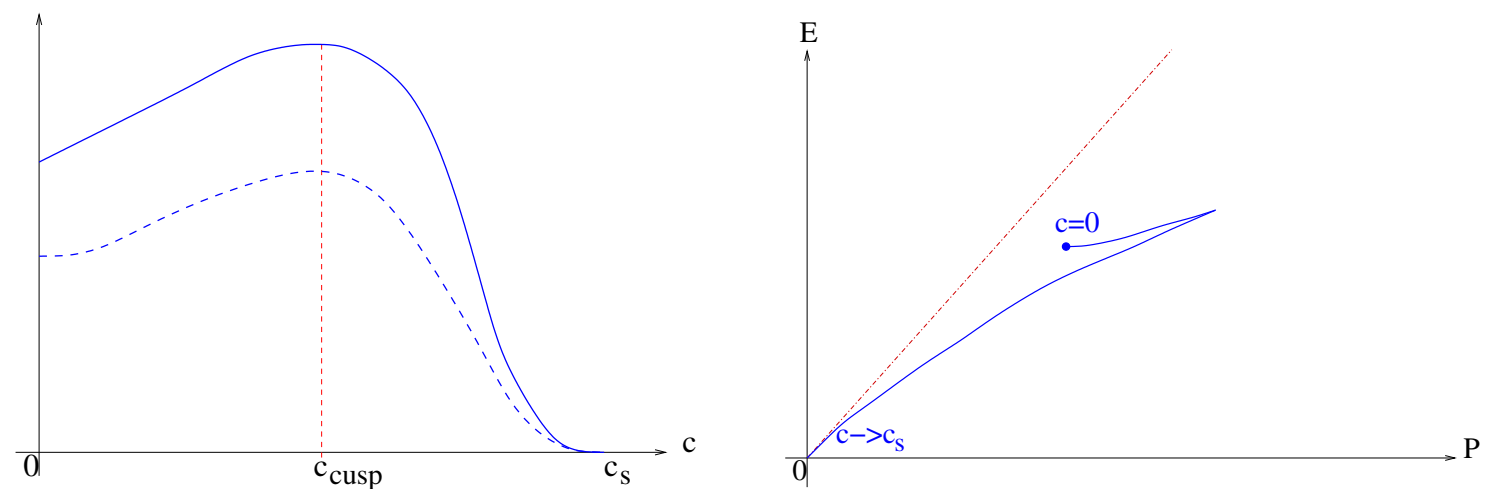

Figure 9. (a) Energy (dashed curve) and momentum (full curve), (b) ( $E, P$ ) diagram.
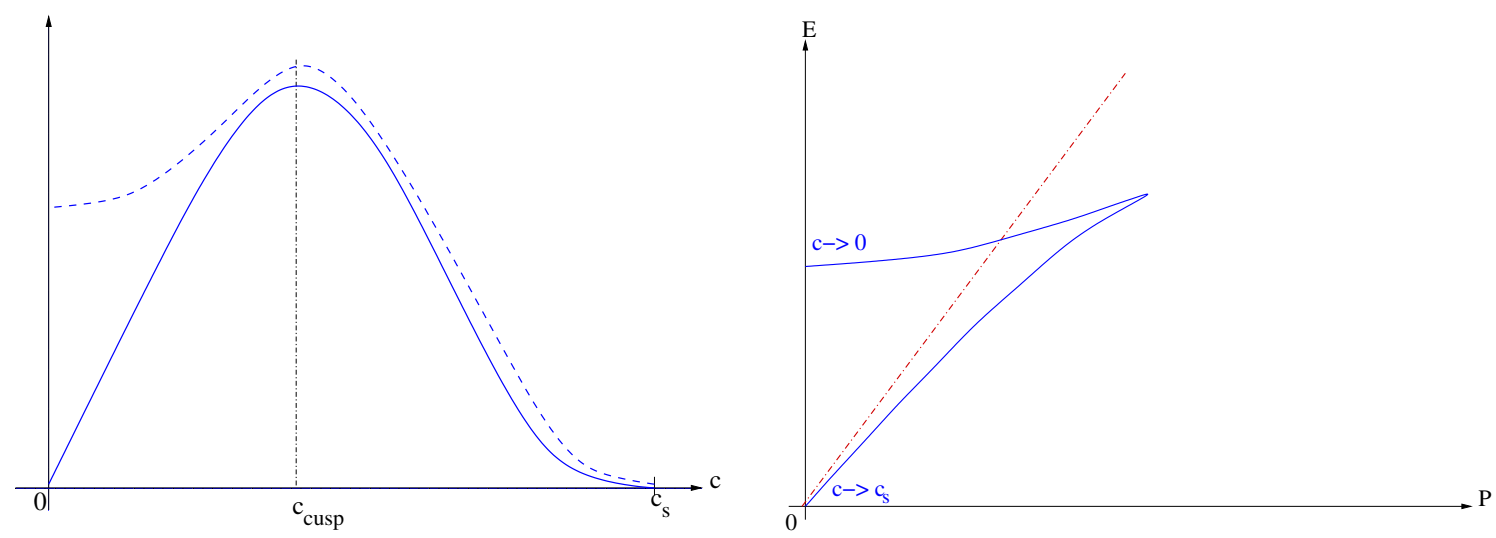

Figure 10. (a) Energy (dashed curve) and momentum (full curve), (b) ( $E, P$ ) diagram.

(i) If $c=0$, then there exists $\phi_{0} \in \mathbb{R}$ such that $\mathrm{e}^{i \phi_{0}} U_{0}$ is a real-valued function and there exist two real constants $M_{0} \neq 0$ (depending only on $f$ and $\xi_{0}$ ) and $x_{0}$ such that, as $x \rightarrow \pm \infty$,

$\mathrm{e}^{i \phi_{0}} U_{0}(x) \mp r_{0} \sim M_{0} \exp \left(-\mathfrak{c}_{s}\left|x-x_{0}\right|\right)$ if $\xi_{0}=-r_{0}^{2}, \quad \mathrm{e}^{i \phi_{0}} U_{0}(x)-r_{0} \sim M_{0} \exp \left(-\mathfrak{c}_{s}\left|x-x_{0}\right|\right)$ if $\xi_{0} \neq-r_{0}^{2}$.

(ii) If $0<c<\mathfrak{c}_{s}$, then $U_{c}$ does not vanish, and hence can be lifted: $U_{c}=A_{c} \mathrm{e}^{i \phi_{c}}$. Furthermore, there exist four real constants $M_{c}, \Theta_{c}$ (depending only on $f, c$ and $\xi_{c}$ ), $x_{0}$ and $\phi_{0}$ such that, as $x \rightarrow \pm \infty$,

$$
\left|U_{c}(x)\right|^{2}-r_{0}^{2}=\eta_{c}(x) \sim \frac{2 r_{0}^{2}}{c} \partial_{x} \phi(x) \sim M_{c} \exp \left(-\sqrt{\mathfrak{c}_{s}^{2}-c^{2}}\left|x-x_{0}\right|\right),
$$

and

$$
\phi(x)-\phi_{0} \mp \Theta_{c} \sim-\operatorname{sgn}(x) \frac{c M_{c}}{2 r_{0}^{2} \sqrt{\mathfrak{c}_{s}^{2}-c^{2}}} \exp \left(-\sqrt{\mathfrak{c}_{s}^{2}-c^{2}}\left|x-x_{0}\right|\right) .
$$


(iii) If $c=\mathfrak{c}_{s}$ then $U_{\mathfrak{c}_{s}}$ does not vanish, and hence can be lifted: $U_{\mathfrak{c}_{s}}=A_{\mathfrak{c}_{s}} \mathrm{e}^{i \phi_{c_{s}}}$. We assume that there exists $m \in \mathbb{N}$ such that $\left(\mathscr{A}_{m}\right)$ is satisfied and define

$$
\Lambda_{m} \equiv \frac{4}{r_{0}^{2(m+1)}}\left[\frac{r_{0}^{2(m+2)}}{(m+3) !} f^{(m+2)}\left(r_{0}^{2}\right)+(-1)^{m+2} \frac{\mathfrak{c}_{s}^{2}}{4}\right] \neq 0 .
$$

Then, we have, as $x \rightarrow \pm \infty$,

and

$$
\left|U_{\mathfrak{c}_{s}}(x)\right|^{2}-r_{0}^{2}=\eta_{\mathfrak{c}_{s}}(x) \sim \frac{2 r_{0}^{2}}{\mathfrak{c}_{s}} \partial_{x} \phi(x) \sim \operatorname{sgn}\left(\xi_{\mathfrak{c}_{s}}\right)\left(\frac{4}{(m+1)^{2}\left|\Lambda_{m}\right| x^{2}}\right)^{\frac{1}{m+1}}
$$

$$
\phi(x) \sim \frac{\mathfrak{c}_{s} \operatorname{sgn}\left(\xi_{\mathfrak{c}_{s}}\right)}{2 r_{0}^{2}}\left(\frac{4}{(m+1)^{2}\left|\Lambda_{m}\right|}\right)^{\frac{1}{m+1}} \begin{cases}\operatorname{sgn}(x) \ln |x| & \text { if } m=1, \\ \frac{m+1}{m-1} \operatorname{sgn}(x)|x|^{\frac{m-1}{m+1}} & \text { if } m \geq 2,\end{cases}
$$

and, if $m=0$, there exist $\Theta_{\mathfrak{c}_{s}} \in \mathbb{R}$ and $\phi_{0} \in \mathbb{R}$ such that

$$
\phi(x)-\phi_{0} \mp \Theta_{\mathfrak{c}_{s}} \sim \operatorname{sgn}\left(\xi_{\mathfrak{c}_{s}}\right) \frac{2 \mathfrak{c}_{s}}{r_{0}^{2}\left|\Lambda_{0}\right| x} .
$$

In particular, since we impose $U_{\mathfrak{c}_{s}} \in \mathscr{L}$, we must have $m \in\{0,1,2\}$.

For the Gross-Pitaevskii nonlinearity $(f(\varrho)=1-\varrho)$, we may compute explicitly the traveling waves for $0<c<\mathfrak{c}_{s}=\sqrt{2}$ (see [Tsuzuki 1971; Béthuel et al. 2008a]):

$$
U_{c}(x)=\sqrt{\frac{2-c^{2}}{2}} \tanh x \frac{\sqrt{2-c^{2}}}{2}-i \frac{c}{\sqrt{2}},
$$

up to the invariances of the problem: translations and multiplications by a phase factor. On this explicit formula, the decay of the phase and modulus can be checked. In particular, as $x \rightarrow \pm \infty$, we have

$$
U_{c}(x) \rightarrow \pm \sqrt{1-\frac{c^{2}}{\mathfrak{c}_{s}}}-i \frac{c}{\mathfrak{c}_{s}} .
$$

Remark 3. In the above statements, the constants $\phi_{0}$ and $x_{0}$ reflect the gauge and translation invariance. In the spirit of the model cases proposed in [Chiron 2012], for

$$
f(\varrho) \equiv-2(\varrho-1)+3(\varrho-1)^{2}-4(\varrho-1)^{3}+5(\varrho-1)^{4}-12(\varrho-1)^{5},
$$

we obtain a smooth decreasing nonlinearity tending to $-\infty$ at $+\infty$ (thus qualitatively similar to the Gross-Pitaevskii nonlinearity) for which we have $r_{0}=1, \mathfrak{c}_{s}=2$, and $\mathscr{V}_{\mathfrak{c}_{s}}(\xi)=-4 \xi^{4}-8 \xi^{5}$. For this nonlinearity $f$, there exists a nontrivial sonic traveling wave of infinite energy (corresponding to $\left.\xi_{\mathfrak{c}_{s}}=-1 / 2\right)$, since $m=3$.

The aim of this paper is to investigate the stability of the traveling waves for the one-dimensional NLS. We recall the definition of orbital stability in a metric space $\left(\mathscr{L}, d_{\mathscr{C}}\right)$ for which we have a local in time existence result. 
Definition 4. Let $0 \leq c \leq \mathfrak{c}_{s}$ and $U_{c} \in \mathscr{L}$ be a nontrivial traveling wave of speed $c$. We say that $U_{c}$ is orbitally stable in $\left(\mathscr{X}, d_{\mathscr{X}}\right)$, where $\mathscr{X} \subset \mathscr{L}$, if, for any $\epsilon>0$, there exists $\delta>0$ such that, for any initial datum $\Psi^{\text {in }} \in \mathscr{X}$ such that $d_{\mathscr{X}}\left(\Psi^{\text {in }}, U_{c}\right) \leq \delta$, any solution $\Psi$ to (NLS) with initial datum $\Psi^{\text {in }}$ is global in $\mathscr{X}$ and

$$
\sup _{t \geq 0} \inf _{\substack{y \in \mathbb{R} \\ \theta \in \mathbb{R}}} d_{\mathscr{R}}\left(\Psi(t), \mathrm{e}^{i \theta} U_{c}(\cdot-y)\right) \leq \epsilon .
$$

In the sequel, $U_{c}$ will always stand for a nontrivial traveling wave, and we freeze the translation invariance by imposing that $\left|U_{c}\right|$ is even. Moreover, the solutions of (NLS) we consider will always be those given by Theorem 1 .

\section{B. Stability and instability in the case $0<c<\mathfrak{c}_{s}$.}

1B1. Stability for the hydrodynamical and the energy distances. The first stability result for the traveling waves for (NLS) with nonzero condition at infinity is due to Z. Lin [2002]. The analysis relies on the hydrodynamical form of (NLS), which is valid for solutions that never vanish. The advantage is to work with a fixed functional space since $(\eta, u)=\left(A^{2}-r_{0}^{2}, \partial_{x} \phi\right) \in H^{1}(\mathbb{R}) \times L^{2}(\mathbb{R})$, whereas the traveling waves have a limit $r_{0} \mathrm{e}^{ \pm i \Theta_{c}}$ (up to a phase factor) at $\pm \infty$ depending on the speed $c$. Lin's result establishes rigorously the stability criterion found in [Bogdan et al. 1989; Barashenkov 1996].

Theorem 5 [Lin 2002]. Assume that $0<c_{*}<\mathfrak{c}_{s}$ is such that there exists a nontrivial traveling wave $U_{c_{*}}$. Then, there exists some small $\sigma>0$ such that $U_{\mathcal{C}_{*}}$ belongs to a locally unique continuous branch of nontrivial traveling waves $U_{c}$ defined for $c_{*}-\sigma \leq c \leq c_{*}+\sigma$.

(i) Assume

$$
{\frac{d P\left(U_{c}\right)}{d c}}_{\mid c=c_{*}}<0 .
$$

Then, $U_{c_{*}}=A_{*} \mathrm{e}^{i \phi_{*}}$ is orbitally stable in the sense that, for any $\epsilon>0$, there exists $\delta>0$ such that, if $\Psi^{\text {in }}=A^{\text {in }} \mathrm{e}^{i \phi^{\text {in }} \in \mathscr{L} \text { satisfies }}$

$$
\left\|A^{\mathrm{in}}-A_{*}\right\|_{H^{1}(\mathbb{R})}+\left\|\partial_{x} \phi^{\mathrm{in}}-\partial_{x} \phi_{*}\right\|_{L^{2}(\mathbb{R})} \leq \delta,
$$

then the solution $\Psi$ to (NLS) such that $\Psi_{\mid t=0}=\Psi^{\mathrm{in}}$ never vanishes, can be lifted to $\Psi=A \mathrm{e}^{i \phi}$, and we have

$$
\sup _{t \geq 0} \inf _{y \in \mathbb{R}}\left\{\left\|A(t)-A_{*}(\cdot-y)\right\|_{H^{1}(\mathbb{R})}+\left\|\partial_{x} \phi(t)-\partial_{x} \phi_{*}(\cdot-y)\right\|_{L^{2}(\mathbb{R})}\right\} \leq \epsilon .
$$

(ii) Assume

$$
{\frac{d P\left(U_{c}\right)}{d c}}_{\mid c=c_{*}}>0 .
$$

Then, $U_{c_{*}}=A_{*} \mathrm{e}^{i \phi_{*}}$ is orbitally unstable in the sense that there exists $\epsilon>0$ such that, for any $\delta>0$, there exists $\Psi^{\text {in }}=A^{\text {in }} \mathrm{e}^{i \phi^{\text {in }}} \in \mathscr{L}$ verifying

$$
\left\|A^{\mathrm{in}}-A_{*}\right\|_{H^{1}(\mathbb{R})}+\left\|\partial_{x} \phi^{\mathrm{in}}-\partial_{x} \phi_{*}\right\|_{L^{2}(\mathbb{R})} \leq \delta,
$$


but such that, if $\Psi$ denotes the solution to (NLS) with $\Psi_{\mid t=0}=\Psi^{\mathrm{in}}$, then there exists $t>0$ such that $\Psi$ does not vanish on the time interval $[0, t]$ but

$$
\inf _{y \in \mathbb{R}}\left\{\left\|A(t)-A_{*}(\cdot-y)\right\|_{H^{1}(\mathbb{R})}+\left\|\partial_{x} \phi(t)-\partial_{x} \phi_{*}(\cdot-y)\right\|_{L^{2}(\mathbb{R})}\right\} \geq \epsilon .
$$

By the one-dimensional Sobolev embedding $H^{1}(\mathbb{R}) \hookrightarrow \mathscr{C}_{0}(\mathbb{R})$, it is clear that, since $U_{c_{*}}$ does not vanish in $\mathbb{R}$, by imposing $\left\|\left|\Psi^{\text {in }}\right|-\left|U_{\mathcal{C}_{*}}\right|\right\|_{H^{1}(\mathbb{R})}=\left\|A^{\text {in }}-A_{*}\right\|_{H^{1}(\mathbb{R})}$ small, $\Psi^{\text {in }}$ does not vanish in $\mathbb{R}$ and thus can be lifted.

Remark 6. We point out that [Gallo 2004] fills two gaps in the proof from [Lin 2002]: the first one concerns the local in time existence for the hydrodynamical system (see (15) in Section 3C) and the second one is about the conservation of the energy and the momentum. Furthermore, we make two additional remarks on the proof from [Lin 2002] in Section 3C.

Theorem 5 is stability or instability in the open set $\mathscr{L}_{\text {hy }} \subset \mathscr{L}$ for the hydrodynamical distance

$$
d_{\text {hy }}(\psi, \tilde{\psi}) \equiv\|A-\tilde{A}\|_{H^{1}(\mathbb{R})}+\left\|\partial_{x} \phi-\partial_{x} \tilde{\phi}\right\|_{L^{2}(\mathbb{R})}+\left|\arg \left(\frac{\psi(0)}{\tilde{\psi}(0)}\right)\right|, \quad \psi=A \mathrm{e}^{i \phi}, \quad \tilde{\psi}=\tilde{A} \mathrm{e}^{i \tilde{\phi}},
$$

which is not the energy distance. Here, arg: $\mathbb{C}^{*} \rightarrow(-\pi,+\pi]$ is the principal argument. For the stability, it suffices to consider the phase $\theta \in \mathbb{R}$ such that $\arg \left(\Psi(t) /\left(\mathrm{e}^{i \theta} U_{c_{*}}(\cdot-y)\right)\right)$ is zero at $x=0$, where $y$ is the translation parameter. For the instability, the phase $\theta \in \mathbb{R}$ does not matter. The result of [Lin 2002] is based on the application of the Grillakis-Shatah-Strauss theory [Grillakis et al. 1987] (see also [Bona et al. 1987; Souganidis and Strauss 1990]) to the hydrodynamical formulation of (NLS) (see Section 3C). One difficulty is to overcome the fact that the Hamiltonian operator $\partial_{x}$ is not onto.

On the energy-momentum diagrams, the stability can be checked either on the graphs of $E$ and $P$ with respect to $c$, or on the concavity of the curve $P \mapsto E$. Indeed, we have seen in [Chiron 2012] that the so-called Hamilton group relation

$$
c=\frac{d E}{d P}, \quad \text { or } \quad \frac{d E\left(U_{c}\right)}{d c}=c \frac{d P\left(U_{c}\right)}{d c},
$$

holds, where the derivative is computed on the local branch. Therefore,

$$
\frac{d^{2} E}{d P^{2}}=\frac{d}{d P} \frac{d E}{d P}=\frac{d c}{d P} .
$$

This means that we have stability when $P \mapsto E$ is concave, that is, $d^{2} E / d P^{2}<0$, and instability if $P \mapsto E$ is convex, i.e., $d^{2} E / d P^{2}>0$.

Actually, the proof of [Grillakis et al. 1987; Lin 2002] provides an explicit control, as shown in the following lemma.

Lemma 7. Under the assumptions of Theorem 5 and in the case (i) of stability, we have, provided $d_{\text {hy }}\left(\Psi^{\text {in }}, U_{c_{*}}\right)$ is small enough,

$$
\begin{aligned}
\sup _{t \geq 0} \inf _{y \in \mathbb{R}}\left\{\left\|A(t)-A_{*}(\cdot-y)\right\|_{H^{1}(\mathbb{R})}+\left\|\partial_{x} \phi(t)-\partial_{x} \phi_{*}(\cdot-y)\right\|_{L^{2}(\mathbb{R})}\right\} \\
\leq K \sqrt{\left|E\left(\Psi^{\mathrm{in}}\right)-E\left(U_{\mathcal{C}_{*}}\right)\right|+\left|P\left(\Psi^{\mathrm{in}}\right)-P\left(U_{c_{*}}\right)\right|},
\end{aligned}
$$


as well as the control

$$
\sup _{t \geq 0} \inf _{\substack{y \in \mathbb{R} \\ \theta \in \mathbb{R}}} d_{\text {hy }}\left(\Psi(t), e^{i \theta} U_{c_{*}}(\cdot-y)\right) \leq K d_{\text {hy }}\left(\Psi^{\text {in }}, U_{c_{*}}\right) .
$$

Remark 8. The second estimate (4) is not a simple consequence of the control (3), but relies on a comparison to $U_{c}$ for some $c$ close to $c_{*}$ instead of a comparison to $U_{c_{*}}$ (this idea has also been used in [Weinstein 1986]). It follows that, in the definition of stability for $U_{c_{*}}$, one can take $\delta=O(\varepsilon)$.

Let us stress that Z. Lin's result (Theorem 5) is given in terms of the hydrodynamical distance $d_{\text {hy }}$, which is not the energy distance $d_{\mathscr{L}}$. As a matter of fact, the Madelung transform

$$
\mathcal{M}:\left(\mathscr{L}_{\mathrm{hy}}, d_{\mathscr{L}}\right) \ni U \mapsto\left(\eta, u, \frac{U(0)}{|U(0)|}\right) \in H^{1}(\mathbb{R}, \mathbb{R}) \times L^{2}(\mathbb{R}, \mathbb{R}) \times \mathbb{S}^{1},
$$

where $U=A \mathrm{e}^{i \phi}, \eta=A^{2}-r_{0}^{2}$ and $u=\partial_{x} \phi$, is not so well behaved.

Lemma 9. (i) The mapping $\mathcal{M}:\left(\mathscr{L}_{\mathrm{hy}}, d_{\mathscr{L}}\right) \rightarrow H^{1}(\mathbb{R}, \mathbb{R}) \times L^{2}(\mathbb{R}, \mathbb{R}) \times \mathbb{S}^{1}$ is an homeomorphism.

(ii) There exists $\phi_{*} \in \mathscr{C}^{2}(\mathbb{R}, \mathbb{R})$ such that $\partial_{x} \phi_{*} \in L^{2}(\mathbb{R})$ and a sequence $\left(\phi_{n}\right)_{n \geq 1}$ of functions in $H^{1}(\mathbb{R}, \mathbb{R})$ such that, when $n \rightarrow+\infty$,

$$
0<d_{\text {hy }}\left(\mathrm{e}^{i \phi_{*}}, \mathrm{e}^{i \phi_{*}} \mathrm{e}^{i \phi_{n}}\right) \rightarrow 0 \quad \text { but } \quad \frac{d_{\mathscr{L}}\left(\mathrm{e}^{i \phi_{*}}, \mathrm{e}^{i \phi_{*}} \mathrm{e}^{i \phi_{n}}\right)}{d_{\mathrm{hy}}\left(\mathrm{e}^{i \phi_{*}}, \mathrm{e}^{i \phi_{*}} \mathrm{e}^{i \phi_{n}}\right)} \rightarrow+\infty .
$$

Therefore, $M^{-1}$ is not locally Lipschitz continuous in general. However, for the stability issues, we compare the $d_{\mathscr{L}}$ and the $d_{\text {hy }}$ distances to some fixed traveling wave $U_{*}$, which enjoys some nice decay properties at infinity. Let us now stress the link between the two distances $d_{\text {hy }}$ and $d_{\mathscr{L}}$ in this case.

Lemma 10. Let $0 \leq c_{*} \leq \mathfrak{c}_{s}$ and assume that $U_{*} \in \mathscr{L}$ is a nonconstant traveling wave with speed $c_{*}$ that does not vanish. If $c_{*}=\mathfrak{c}_{s}$, we further assume that assumption $\left(\mathscr{A}_{0}\right)$ is satisfied. Then, there exists some constants $K$ and $\delta>0$, depending only on $U_{*}$, such that, for any $\psi \in \mathscr{L}$ verifying $d_{\mathscr{L}}\left(\psi, U_{*}\right) \leq \delta$, we have

$$
\frac{1}{K} d_{\text {hy }}\left(\psi, U_{*}\right) \leq d_{\mathscr{L}}\left(\psi, U_{*}\right) \leq K d_{\text {hy }}\left(\psi, U_{*}\right) .
$$

An immediate corollary of Lemma 10 is that Theorem 5 is also a stability/instability result in the energy distance. If one wishes for only a stability/instability result, it is sufficient to invoke the fact that the mapping $\mathcal{M}$ is an homeomorphism. However, the use of Lemma 10 provides a stronger explicit control similar to the one obtained in Lemma 7 (see (3)). In particular, in the definition of stability for $U_{c_{*}}$ in $\left(\mathscr{E}, d_{\mathscr{L}}\right)$, one can take $\delta=\mathcal{O}(\varepsilon)$.

Corollary 11. Assume that $0<c_{*}<\mathfrak{c}_{s}$ is such that there exists a nontrivial traveling wave $U_{c_{*}}$. Then, there exists some small $\sigma>0$ such that $U_{c_{*}}$ belongs to a locally unique continuous branch of nontrivial traveling waves $U_{c}$ defined for $c_{*}-\sigma \leq c \leq c_{*}+\sigma$.

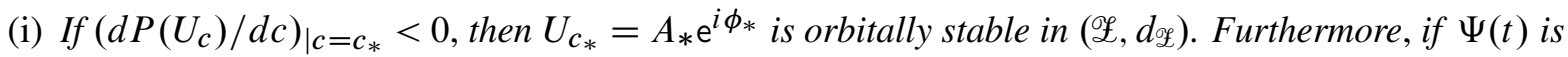
the (global) solution to (NLS) with initial datum $\Psi^{\text {in }}$, then we have, for some constant $K$ depending 
only on $U_{c_{*}}$ and provided $d_{\mathscr{E}}\left(\Psi^{\mathrm{in}}, U_{\mathcal{C}_{*}}\right)$ is sufficiently small,

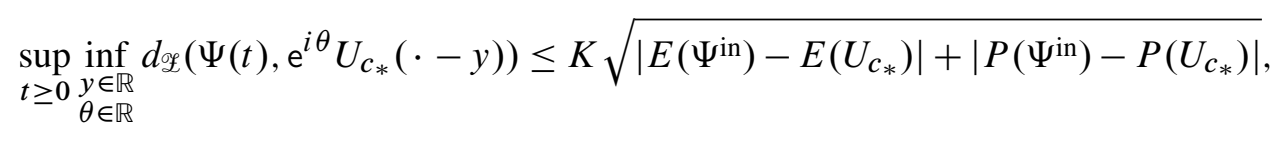

as well as the control

$$
\sup _{t \geq 0} \inf _{\substack{y \in \mathbb{R} \\ \theta \in \mathbb{R}}} d_{\mathscr{L}}\left(\Psi(t), \mathrm{e}^{i \theta} U_{c_{*}}(\cdot-y)\right) \leq K d_{\mathscr{E}}\left(\Psi^{\mathrm{in}}, U_{c_{*}}\right) .
$$

(ii) If $\left(d P\left(U_{c}\right) / d c\right)_{\mid c=c_{*}}>0$, then $U_{c_{*}}=A_{*} \mathrm{e}^{i \phi_{*}}$ is orbitally unstable in ( $\left.\mathscr{L}, d_{\mathscr{E}}\right)$.

For the Gross-Pitaevskii nonlinearity $(f(\varrho)=1-\varrho)$, the stability (for the energy distance $d_{\mathscr{L}}$ ) of the traveling waves with speed $0<c<\mathfrak{c}_{s}$ was proved by F. Béthuel, P. Gravejat and J.-C. Saut [Béthuel et al. 2008a] through the variational characterization that these solutions are minimizers of the energy under the constraint of fixed momentum. However, in view of the energy momentum diagrams in Section 1A, this constraint minimization approach can not be used in the general setting we consider here. Indeed, this method provides only stability, but there may exist unstable traveling waves. Moreover, it follows from the proof of Theorem 5 that stable waves are local minimizers of the energy at fixed momentum but not necessarily global minimizers. Finally, we emphasize that the spectral methods allow us to derive an explicit (Lipschitz) control in case of stability.

1B2. Stability via a Liapounov functional. Another way to prove the orbital stability is to find a Liapounov functional. By Liapounov functional, we mean a functional which is conserved by the (NLS) flow and for which the traveling wave $U_{c}$ is a local minimum (for instance, a critical point with second derivative $\geq \delta$ Id for some $\delta>0$ ). Such a Liapounov functional always exists in the Grillakis-Shatah-Strauss theory when $\left(d P\left(U_{c}\right) / d c\right)_{\mid c=c_{*}}<0$, as shown by Theorem 26 in Appendix A. Its direct application to our problem leads us to define the functional in $\mathscr{L}_{\text {hy }}$

$$
\mathscr{L}(\psi) \equiv E(\psi)-c_{*} P(\psi)+\frac{M}{2}\left(P(\psi)-P\left(U_{c_{*}}\right)\right)^{2},
$$

where $M$ is some positive parameter. It turns out that $\mathscr{L}$ is such a Liapounov functional when $M$ is sufficiently large. Since the proof relies on the Grillakis-Shatah-Strauss framework, we have to work in the hydrodynamical variables. However, by Lemma 10, we recover the case of the energy distance.

Theorem 12. Assume that, for some $c_{*} \in\left(0, \mathfrak{c}_{s}\right)$ and $\sigma>0$ small, $\left(0, \mathfrak{c}_{s}\right) \supset\left[c_{*}-\sigma, c_{*}+\sigma\right] \ni c \mapsto U_{c} \in \mathscr{Z}$ is a continuous branch of nontrivial traveling waves with $\left(d P\left(U_{c}\right) / d c\right)_{\mid c=c_{*}}<0$. If

$$
M>\frac{1}{-\frac{d P\left(U_{c}\right)}{d c} \mid c=c_{*}}>0,
$$

there exist $\epsilon>0$ and $K$, depending only on $U_{\mathcal{C}_{*}}$, such that, for any $\psi \in \mathscr{Z}$ with

$$
\inf _{y \in \mathbb{R}, \theta \in \mathbb{R}} d_{\text {hy }}\left(\psi, \mathrm{e}^{i \theta} U_{c_{*}}(\cdot-y)\right) \leq \epsilon,
$$


we have

$$
\inf _{\substack{y \in \mathbb{R} \\ \theta \in \mathbb{R}}} d_{\mathrm{hy}}^{2}\left(\psi, \mathrm{e}^{i \theta} U_{c_{*}}(\cdot-y)\right) \leq K\left(\mathscr{L}(\psi)-\mathscr{L}\left(U_{c_{*}}\right)\right),
$$

and analogously with $d_{\mathrm{hy}}$ replaced by $d_{\mathscr{L}}$. Consequently, $U_{c_{*}}=A_{*} \mathrm{e}^{i \phi_{*}}$ is orbitally stable in $\left(\mathscr{L}_{\mathrm{hy}}, d_{\mathrm{hy}}\right)$ and in $\left(\mathscr{L}, d_{\mathscr{L}}\right)$. Furthermore, if $\Psi(t)$ is the (global) solution to (NLS) with initial datum $\Psi^{\mathrm{in}}$, then we have

$$
\sup _{t \geq 0} \inf _{\substack{y \in \mathbb{R} \\ \theta \in \mathbb{R}}} d_{\mathrm{hy}}\left(\Psi(t), \mathrm{e}^{i \theta} U_{c_{*}}(\cdot-y)\right) \leq K \sqrt{\mathscr{L}\left(\Psi^{\mathrm{in}}\right)-\mathscr{L}\left(U_{c_{*}}\right)} \leq K d_{\mathrm{hy}}\left(\Psi^{\mathrm{in}}, U_{c_{*}}\right),
$$

provided $d_{\mathrm{hy}}\left(\Psi^{\mathrm{in}}, U_{c_{*}}\right)$ is sufficiently small, and analogously with $d_{\mathrm{hy}}$ replaced by $d_{\mathscr{L}}$.

For the traveling waves for (NLS) in dimension one, this type of Liapounov functional appears for the first time in the paper by I. Barashenkov [1996]. However, there, the problem is treated directly on the wave function $\Psi$, whereas the correct proof holds on the hydrodynamical variables, in particular because of the gauge invariance $(\theta, \Psi) \mapsto \mathrm{e}^{i \theta} \Psi$. For instance, that work suggests that we have stability for $H^{1}$ perturbations, whereas it holds only for perturbations in the energy space. Finally, we fill some gaps in the proof of [Barashenkov 1996].

1B3. Instability via the existence of an unstable eigenvalue. In the Grillakis-Shatah-Strauss theory [Grillakis et al. 1987], the instability is not shown by proving the existence of a unstable eigenvalue for the linearized (NLS) and then a nonlinear instability result (see however [Grillakis et al. 1990] when the Hamiltonian skew-adjoint operator is onto). There exist, however, some general results that prove the existence of unstable eigenvalues. For the instability of bound states for (NLS) (and also for the nonlinear Klein-Gordon equation), that is, solutions of the form $\mathrm{e}^{i \omega t} U_{\omega}(x), \mathrm{M}$. Grillakis [1988] shows that the condition $d / d \omega\left(\int_{\mathbb{R}^{d}}\left|U_{\omega}\right|^{2}\right)_{\mid \omega=\omega_{*}}>0$ is sufficient for the existence of such an unstable eigenvalue. However, the proof relies on the fact that the bound states are real-valued functions (up to a phase factor) and it is not clear whether it extends to the case of traveling waves we are studying. Indeed, since we have to work in hydrodynamical variables in order to have a fixed functional space, the linearized operator does not have (for $c \neq 0$ ) the structure required for the application of [Grillakis 1988]. Another general result is due to O. Lopes [2002] but it assumes that the linearized equation can be solved using a semigroup. This is not the case for our problem once it is written in hydrodynamical variables (see below). Finally, Z. Lin [2008] proposes an alternative approach for the existence of unstable eigenvalues. The method has the advantage of allowing pseudodifferential equations (like the Benjamin-Ono equation). However, the results are given for three model equations involving a scalar unknown, and it is not clear whether the proof can be extended to the case of systems.

The linearization of (NLS) near the traveling wave $U_{c_{*}}$ in the frame moving with speed $c_{*}$ is

$$
i \frac{\partial \psi}{\partial t}-i c_{*} \partial_{x} \psi+\partial_{x}^{2} \psi+\psi f\left(\left|U_{c_{*}}\right|^{2}\right)+2\left\langle\psi \mid U_{c_{*}}\right\rangle f^{\prime}\left(\left|U_{c_{*}}\right|^{2}\right) U_{c_{*}}=0,
$$

and, thus, searching for exponentially growing modes $\psi(t, x)=\mathrm{e}^{\lambda t} w(x)$ leads to the eigenvalue problem

$$
i \lambda w-i c_{*} \partial_{x} w+\partial_{x}^{2} w+w f\left(\left|U_{c_{*}}\right|^{2}\right)+2\left\langle w \mid U_{c_{*}}\right\rangle f^{\prime}\left(\left|U_{c_{*}}\right|^{2}\right) U_{c_{*}}=0,
$$


with $\operatorname{Re}(\lambda)>0$ and $w \neq 0$. For one-dimensional problems, the linear instability is commonly shown through the use of Evans functions (see the classical paper [Pego and Weinstein 1992] and also the review article [Sandstede 2002]). For our problem, we look for an unstable eigenvalue for the equation written in hydrodynamical variables; namely we look for exponentially growing solutions $(\eta, \underline{u})$ of the linear problem (written in the moving frame)

$$
\left\{\begin{array}{l}
\partial_{t} \underline{\eta}-c_{*} \partial_{x} \underline{\eta}+2 \partial_{x}\left(\left(r_{0}^{2}+\eta_{*}\right) \underline{u}+\underline{\eta} u_{*}\right)=0, \\
\partial_{t} \underline{u}-c_{*} \partial_{x} \underline{u}+2 \partial_{x}\left(u_{*} \underline{u}\right)-\partial_{x}\left(f^{\prime}\left(r_{0}^{2}+\eta_{*}\right) \underline{\eta}\right) \\
\quad-\partial_{x}\left\{\frac{1}{2 \sqrt{r_{0}^{2}+\eta_{*}}} \partial_{x}^{2}\left(\frac{\underline{\eta}}{\sqrt{r_{0}^{2}+\eta_{*}}}\right)-\frac{\eta \partial_{x}^{2}\left(\sqrt{r_{0}^{2}+\eta_{*}}\right)}{2\left(r_{0}^{2}+\eta_{*}\right)^{3 / 2}}\right\}=0,
\end{array}\right.
$$

where $\left(\eta_{*}, u_{*}\right)$ is the reference solution. The advantage is here again to work with a fixed functional space in variables $(\eta, u)$. Due to the term

$$
\partial_{x}\left\{\frac{1}{2 \sqrt{r_{0}^{2}+\eta_{*}}} \partial_{x}^{2}\left(\frac{\underline{\eta}}{\sqrt{r_{0}^{2}+\eta_{*}}}\right)\right\},
$$

this equation can not be solved using a semigroup, except in the trivial case where $\eta_{*}$ is constant; hence the result of [Lopes 2002] does not apply. However, system (7) is a particular case of the Euler-Korteweg system for capillary fluids (see [Benzoni-Gavage 2010a] for a survey on this model). We may then use a linear instability result already shown for the Euler-Korteweg system with the Evans function method, as in work by K. Zumbrun [2008] for a simplified system, and more recently by S. Benzoni-Gavage [2010b] for the complete Euler-Korteweg system.

Theorem 13. Assume that, for some $c_{*} \in\left(0, \mathfrak{c}_{s}\right)$ and $\sigma>0$ small,

$$
\left(0, \mathfrak{c}_{s}\right) \supset\left[c_{*}-\sigma, c_{*}+\sigma\right] \ni c \mapsto U_{c} \in \mathscr{L}
$$

is a continuous branch of nontrivial traveling waves with

$$
{\frac{d P\left(U_{c}\right)}{d c} \mid c=c_{*}}>0 .
$$

Then, there exists exactly one unstable eigenvalue $\gamma_{0} \in\{\operatorname{Re}>0\}$ for $(6)$ and $\gamma_{0} \in(0,+\infty)$; that is, (NLS) is (spectrally) linearly unstable.

Once we have shown the existence of an unstable eigenvalue for the linearized NLS equation (5), we can prove a nonlinear instability result as in [Henry et al. 1982; de Bouard 1995]. Note that, here, we no longer work in the hydrodynamical variables, where the high-order derivatives involve nonlinear terms, but on the semilinear NLS equation.

Corollary 14. Under the assumptions of Theorem $13, U_{c_{*}}$ is unstable in $U_{c_{*}}+H^{1}(\mathbb{R}, \mathbb{C}$ ) (endowed with the natural $H^{1}$ distance): there exists $\epsilon$ such that, for any $\delta>0$, there exists $\Psi^{\text {in }} \in U_{c_{*}}+H^{1}(\mathbb{R})$ such that $\left\|\Psi^{\mathrm{in}}-U_{c_{*}}\right\|_{H^{1}(\mathbb{R})} \leq \delta$, but, if $\Psi \in U_{c_{*}}+\mathscr{C}\left(\left[0, T^{*}\right), H^{1}(\mathbb{R})\right)$ denotes the maximal solution of (NLS), then there exists $0<t<T^{*}$ such that $\left\|\Psi(t)-U_{c_{*}}\right\|_{H^{1}(\mathbb{R})} \geq \epsilon$. 
Since the proof is very similar to the one in [Henry et al. 1982; de Bouard 1995], we omit it. We may actually prove a stronger instability result, since the above one is not proved by tracking the exponentially growing mode. In [Di Menza and Gallo 2007], a spectral mapping theorem is shown and used to show the nonlinear instability by tracking this exponentially growing mode, which is a natural mechanism of instability. In Appendix B, we show that this spectral mapping theorem holds for a wide class of Hamiltonian equations. The direct application of Corollary B.6 in Appendix B gives the following nonlinear instability result.

Corollary 15. We make the assumptions of Theorem 13, so that there exists an unstable eigenmode $\left(\gamma_{0}, w\right) \in(0,+\infty) \times H^{1}(\mathbb{R}),\|w\|_{H^{1}}=1$. There exists $M>0$ such that, for any solution $\psi \in$ $\mathscr{C}\left(\mathbb{R}_{+}, H^{1}(\mathbb{R}, \mathbb{C})\right)$ of the linearized equation $(5)$, we have the growth estimate of the semigroup

$$
\|\psi(t)\|_{H^{1}(\mathbb{R})} \leq M \mathrm{e}^{\gamma_{0} t}\|\psi(0)\|_{H^{1}(\mathbb{R})} \text { for all } t \geq 0 .
$$

Moreover, $U_{\mathcal{C}_{*}}$ has also the following instability property: there exist $K>0, \delta>0$ and $\varepsilon_{0}>0$, such that, for any $0<\delta<\delta_{0}$, the solution $\Psi(t)$ to $(\mathrm{NLS})$ with initial datum $\Psi^{\mathrm{in}}=U_{c_{*}}+\delta w \in U_{c_{*}}+H^{1}(\mathbb{R})$ exists at least on $\left[0, \gamma_{0}^{-1} \ln \left(2 \varepsilon_{0} / \delta\right)\right]$ and satisfies

$$
\left\|\Psi(t)-U_{c_{*}}-\delta \mathrm{e}^{\gamma_{0} t} w\right\|_{H^{1}(\mathbb{R})} \leq K \delta^{2} \mathrm{e}^{2 \gamma_{0} t} .
$$

In particular, for $t=\gamma_{0}^{-1} \ln \left(2 \varepsilon_{0} / \delta\right)$ and $\epsilon \equiv \varepsilon_{0} / K$, we have

$$
\inf _{y \in \mathbb{R}}\left\||\Psi(t)|-\left|U_{c_{*}}\right|(\cdot-y)\right\|_{L^{2}(\mathbb{R})} \geq \epsilon \quad \text { and } \quad \inf _{y \in \mathbb{R}}\left\||\Psi(t)|-\left|U_{c_{*}}\right|(\cdot-y)\right\|_{L^{\infty}(\mathbb{R})} \geq \epsilon,
$$

which implies

$$
\inf _{\substack{y \in \mathbb{R} \\ \theta \in \mathbb{R}}}\left\|\Psi(t)-\mathrm{e}^{i \theta} U_{c_{*}}(\cdot-y)\right\|_{H^{1}(\mathbb{R})} \geq \epsilon
$$

as well as

$$
\inf _{\substack{y \in \mathbb{R} \\ \theta \in \mathbb{R}}} d_{\mathrm{hy}}\left(\Psi(t), \mathrm{e}^{i \theta} U_{c_{*}}(\cdot-y)\right) \geq \epsilon \quad \text { and } \quad \inf _{\substack{y \in \mathbb{R} \\ \theta \in \mathbb{R}}} d_{\mathscr{L}}\left(\Psi(t), \mathrm{e}^{i \theta} U_{c_{*}}(\cdot-y)\right) \geq \epsilon .
$$

With the above result, we then show the nonlinear instability also in the energy space, and thus recover the instability result of $\mathrm{Z}$. Lin but this time by tracking the unstable growing mode.

1B4. Instability at a cusp. In this section, we investigate the question of stability in the degenerate case $d P / d c=0$. In [Grillakis et al. 1987] (see also [Grillakis et al. 1990]), a stability result for the wave of speed $c_{*}$ is shown when the action $c \mapsto S(c)=E\left(U_{c}\right)-c P\left(U_{c}\right)$ (on the local branch) is such that, for instance, $d^{2} S / d c^{2}=-d P / d c$ is positive for $c \neq c_{*}$ but vanishes for $c=c_{*}$. In the energy-momentum diagrams of Section 1A, the situation is different since $d P / d c$ changes sign at the cusps, or, equivalently, the action $c \mapsto S(c)=E\left(U_{c}\right)-c P\left(U_{c}\right)$ (on the local branch) changes its concavity at the cusp. A. Comech and D. Pelinovsky [2003] show that, for the nonlinear Schrödinger equation, a bound state associated with a cusp in the energy-charge diagram is unstable. The proof relies on a careful analysis of the linearized equation, which is spectrally stable, but linearly unstable (with polynomial growth for the linear problem). A similar technique was used by A. Comech, S. Cuccagna and D. Pelinovsky [2007] for the generalized 
Korteweg-de Vries equation. Then, M. Ohta [2011] also proved the nonlinear instability of these "bound states" using a Liapounov functional as in [Grillakis et al. 1987]. However, in [Ohta 2011], it is assumed that $J=T^{\prime}(0)$ and that $J$ is onto, which are both not true here (and there are further restrictions due to the introduction of an intermediate Hilbert space). M. Maeda [2012] has extended the above instability result, removing some assumptions in [Ohta 2011]. We show the instability of traveling waves associated with a cusp in the energy-momentum diagram in the generic case where $d^{2} P / d c^{2} \neq 0$. Our approach follows the lines of [Maeda 2012], but with some modifications since our problem does not fit exactly the general framework of this paper. In particular, we can not find naturally a space " $Y$ ", and some functions appearing in the proof do not lie in the range of the skew-adjoint operator $\partial_{x}$ involved in the Hamiltonian formalism. We overcome this difficulty using an approximation argument (similar to the one used in [Lin 2002]).

Theorem 16. Assume that, for some $c_{*} \in\left(0, \mathfrak{c}_{s}\right)$ and $\sigma>0$ small, $\left(0, \mathfrak{c}_{s}\right) \supset\left[c_{*}-\sigma, c_{*}+\sigma\right] \ni c \mapsto U_{c} \in \mathscr{L}$ is a continuous branch of nontrivial traveling waves with

$$
{\frac{d P\left(U_{c}\right)}{d c}}_{\mid c=c_{*}}=0 \neq{\frac{d^{2} P\left(U_{c}\right)}{d c^{2}} \mid c=c_{*}},
$$

and assume in addition that $f$ is of class $\mathscr{C}^{2}$. Then, $U_{\mathcal{C}_{*}}$ is orbitally unstable in $\left(\mathscr{L}, d_{\mathscr{L}}\right)$.

\section{C. Stability in the case $c=0$.}

1C1. Instability for the bubbles. When $c=0$, we have two types of stationary waves: the bubbles, when $\xi_{0}>-r_{0}^{2}$, are even functions (up to a translation) that do not vanish, and the kinks, when $\xi_{0}=-r_{0}^{2}$, are odd functions (up to a translation). The instability of stationary bubbles has been shown by A. de Bouard [1995] (and is true even in higher dimension). The proof there relies on the proof of the existence of an unstable eigenvalue for the linearized NLS, and then the proof of a nonlinear instability result. An alternative proof of the linear instability of the bubbles is given in [Pelinovsky and Kevrekidis 2008, Theorem 3.11(ii)].

Theorem 17 [de Bouard 1995]. Assume that there exists a bubble, that is, a nontrivial stationary $(c=0)$ wave $U_{0}$ which does not vanish. Then, $U_{0}$ is (linearly and nonlinearly) unstable in $U_{0}+H^{1}(\mathbb{R})$ (endowed with the natural $H^{1}$ metric); that is, there exists $\epsilon$ such that, for any $\delta>0$, there exists $\Psi^{\mathrm{in}} \in U_{0}+H^{1}(\mathbb{R})$ such that $\left\|\Psi^{\mathrm{in}}-U_{0}\right\|_{H^{1}(\mathbb{R})} \leq \delta$, but, if $\Psi \in U_{0}+\mathscr{C}\left(\left[0, T^{*}\right), H^{1}(\mathbb{R})\right)$ denotes the maximal solution of (NLS), then there exists $0<t<T^{*}$ such that $\left\|\Psi(t)-U_{0}\right\|_{H^{1}(\mathbb{R})} \geq \epsilon$.

Actually, in the same way that Corollary 15 is a better instability result than Corollary 14, we have the following stronger instability result, which is a direct consequence of Corollary B.6 in Appendix B.

Proposition 18. Assume that there exists a bubble, that is, a nontrivial stationary $(c=0)$ wave $U_{0}$ which does not vanish. Then, $U_{0}$ is (nonlinearly) unstable in $U_{0}+H^{1}(\mathbb{R}),\left(\mathscr{E}, d_{\mathscr{E}}\right)$ and $\left(\mathscr{L}_{\mathrm{hy}}, d_{\mathrm{hy}}\right)$ in the same sense as in Corollary 15.

Finally, we would like to emphasize that we may recover the instability result for bubbles from the proof of Theorem 5, relying on the hydrodynamical form of (NLS), which holds true here since bubbles do not vanish. Our result holds in the energy space and for the hydrodynamical distance. 
Theorem 19. Assume that there exists a bubble, that is, a nontrivial stationary $(c=0)$ wave $U_{0}$ which does not vanish. Then, there exists some small $\sigma>0$ such that $U_{0}$ belongs to a locally unique continuous branch of nontrivial traveling waves $U_{c}$ defined for $0 \leq c \leq \sigma$. Then, $c \mapsto P\left(U_{c}\right)$ has a derivative at $c=0$,

$$
\left.\frac{d P\left(U_{c}\right)}{d c}\right|_{\mid c=0}>0
$$

and $U_{0}=A_{*} \mathrm{e}^{i \phi_{*}}$ is orbitally unstable for the distances $d_{x}$ and $d_{\mathrm{hy}}$.

Proof. We give a proof based on the argument of [Lin 2002], which is possible since $U_{0}$ is a bubble, hence does not vanish, and the spectral decomposition used there still holds when $c=0$. Moreover, it is clear that the mapping $c \mapsto\left(\eta_{c}, u_{c}\right) \in H^{1} \times L^{2}$ is smooth up to $c=0$, using the uniform exponential decay at infinity near $c=0$ and arguing as in [Chiron 2012]. Therefore, it suffices to show that $\left(d P\left(U_{c}\right) / d c\right)_{\mid c=0}>0$. From the expression of the momentum given in [ibid., Subsection 1.2], we have, for $0 \leq c \leq \sigma$,

$$
P\left(U_{c}\right)=c \operatorname{sgn}\left(\xi_{c}\right) \int_{0}^{\xi_{c}} \frac{\xi^{2}}{r_{0}^{2}+\xi} \frac{d \xi}{\sqrt{-V_{c}(\xi)}}=c\left|\int_{0}^{\xi_{0}} \frac{\xi^{2}}{r_{0}^{2}+\xi} \frac{d \xi}{\sqrt{-\mathscr{V}_{0}(\xi)}}\right|+o(c)
$$

since $\xi_{0}>-r_{0}^{2}$. Indeed, we are allowed to pass to the limit in the integral once it is written with the change of variables $\xi=t \xi_{c}$ :

$$
\int_{0}^{\xi_{c}} \frac{\xi^{2}}{r_{0}^{2}+\xi} \frac{d \xi}{-V_{c}(\xi)}=\int_{0}^{1} \frac{\xi_{c}^{3} t^{2}}{r_{0}^{2}+t \xi_{c}} \frac{d t}{\sqrt{-V_{c}\left(t \xi_{c}\right)}}
$$

since $\xi_{0}>-r_{0}^{2}$. Therefore,

$$
\left.\frac{d P\left(U_{c}\right)}{d c}\right|_{\mid c=0}=\left|\int_{0}^{\xi_{0}} \frac{\xi^{2}}{r_{0}^{2}+\xi} \frac{d \xi}{\sqrt{-V_{0}(\xi)}}\right|>0
$$

since $\xi_{0} \neq 0$ ( $U_{0}$ is not trivial). The conclusion follows then from the proof of Theorem 5 .

When we know that $\left(d P\left(U_{c}\right) / d c\right)_{\mid c=0}>0$, we may also use the Evans function as in Theorem 13 to show the existence of an unstable eigenmode. However, due to the fact that the kink $U_{0}$ is real-valued, we can use the arguments in [de Bouard 1995; Pelinovsky and Kevrekidis 2008].

1C2. Stability analysis for the kinks. We now turn to the case of the kinks $\left(\xi_{0}=-r_{0}^{2}\right.$ and $U_{0}$ is odd up to a translation). Since $U_{0}$ vanishes at the origin, the hydrodynamical form of (NLS) can not be used. The stability of the kink has attracted several recent works. L. Di Menza and C. Gallo [2007] have investigated the linear stability through the Vakhitov-Kolokolov function VK, defined by

$$
\operatorname{VK}(\lambda) \equiv \int_{\mathbb{R}}\left(\left[-\partial_{x}^{2}-f\left(U_{0}^{2}\right)-\lambda\right]^{-1}\left(\partial_{x} U_{0}\right)\right)\left(\partial_{x} U_{0}\right) d x,
$$

where $U_{0}$ is the kink, for $\lambda \in\left(\lambda_{*}, 0\right)$ for some $\lambda_{*}<0$. They show that the Vakhitov-Kolokolov function VK has a limit $\mathrm{VK}_{0}$ when $\lambda \rightarrow 0^{-}$. If $\mathrm{VK}_{0}>0$, then the linearization of (NLS) around the kink has an unstable real positive eigenvalue. When $\mathrm{VK}_{0}<0$, the linearization of (NLS) around the kink has a spectrum included in $i \mathbb{R}$ (spectral stability). Note that the approach of [Lin 2002] (extending [Grillakis 
et al. 1987]) does not give directly unstable eigenvalues in the case $d P / d c>0$. Recently, the link between the quantity $d P / d c$ and the sign of $\mathrm{VK}_{0}$ has been given by D. Pelinovsky and P. Kevrekidis [2008] (proof of Lemma 3.10 there, the factor $\sqrt{2}$ coming from the coefficients of the NLS equation in [Pelinovsky and Kevrekidis 2008]):

$$
2 \sqrt{2} \mathrm{VK}_{0}=\lim _{c \rightarrow 0} \frac{d P\left(U_{c}\right)}{d c},
$$

and they also prove, in a different way from [Di Menza and Gallo 2007], that we have spectral stability when $\lim _{c \rightarrow 0} d P / d c<0$ and existence of an unstable eigenvalue (in $\mathbb{R}_{+}^{*}$ ) if $\lim _{c \rightarrow 0} d P / d c>0$. It is shown in [Pelinovsky and Kevrekidis 2008] that the $\operatorname{limit}_{\lim _{c \rightarrow 0}} d P\left(U_{c}\right) / d c$ does exist. Actually, it is proved there that the function $\left[0, c_{0}\right) \ni c \mapsto P\left(U_{c}\right)$ is of class $\mathscr{C}^{1}$ and that the derivative at $c=0$ is also given by (see (8))

$$
\begin{aligned}
\lim _{c \rightarrow 0} \frac{d P\left(U_{c}\right)}{d c} & =2 \sqrt{2} \mathrm{VK}_{0}=2 \sqrt{2} \lim _{\lambda \rightarrow 0^{-}} \int_{\mathbb{R}}\left(\left[-\partial_{x}^{2}-f\left(U_{0}^{2}\right)-\lambda\right]^{-1}\left(\partial_{x} U_{0}\right)\right)\left(\partial_{x} U_{0}\right) d x \\
& =2 \sqrt{2} \int_{\mathbb{R}} \operatorname{Im}\left(\frac{\partial U_{c}}{\partial c}{ }_{\mid c=0}\right) \partial_{x} U_{0} d x .
\end{aligned}
$$

Our next lemma gives an explicit formula of the expression (9), involving only the nonlinearity $f$.

Lemma 20. Assume that $U_{0}$ is a kink. Then, there exists $c_{0} \in\left(0, \mathfrak{c}_{s}\right)$ such that $U_{0}$ belongs to the (locally) unique branch $\left[0, c_{0}\right) \ni c \mapsto U_{c} \in \mathscr{L}$. Moreover, $P\left(U_{c}\right) \rightarrow r_{0}^{2} \pi$ as $c \rightarrow 0$ and the continuous extension $\left[0, c_{0}\right) \ni c \mapsto P\left(U_{c}\right)$ has a derivative at $c=0$ given by

$$
{\left.\frac{d P\left(U_{c}\right)}{d c}\right|_{\mid c=0}}=-\frac{8 r_{0}^{3}}{3 \sqrt{F(0)}}+\frac{1}{2} \int_{0}^{r_{0}^{2}} \frac{\left(\varrho-r_{0}^{2}\right)^{2}}{\varrho^{3 / 2}}\left(\frac{1}{\sqrt{F(\varrho)}}-\frac{1}{\sqrt{F(0)}}\right) d \varrho .
$$

The advantage of the formula given in Lemma 20 compared to (9) is that it allows a direct computation of $\left(d P\left(U_{c}\right) / d c\right)_{\mid c=0}$ when $f$ is known, which does not require computing numerically $U_{0}$ and $\left(\partial U_{c} / \partial c\right)_{\mid c=0}$. For instance, it is quite well adapted to the stability analysis as in [Fakau and Karval'u 2009]. Let us observe that it may happen that a kink is unstable (see [Kivshar and Krolikowski 1995; Di Menza and Gallo 2007]).

In the case of linear instability, [Di Menza and Gallo 2007] shows that, then, nonlinear instability holds. Actually, a stronger result is proved there, showing that the $L^{\infty}$ norm (and not only the $H^{1}$ norm) does not remain small.

Theorem 21 [Di Menza and Gallo 2007]. Assume that there exists a kink, that is, a nontrivial stationary $(c=0)$ wave $U_{0}$ vanishing somewhere, and satisfying $\left(d P\left(U_{c}\right) / d c\right)_{\mid c=0}>0$. Then, $U_{0}$ is (linearly and nonlinearly) unstable in the sense that there exists $\epsilon$ such that, for any $\delta>0$, there exists $\Psi^{\text {in }} \in U_{0}+H^{1}(\mathbb{R})$ such that $\left\|\Psi^{\mathrm{in}}-U_{0}\right\|_{H^{1}(\mathbb{R})} \leq \delta$, but, if $\Psi \in U_{0}+\mathscr{C}\left(\left[0, T^{*}\right), H^{1}(\mathbb{R})\right)$ denotes the maximal solution of (NLS), then there exists $0<t<T^{*}$ such that $\left\|\Psi(t)-U_{0}\right\|_{L^{\infty}(\mathbb{R})} \geq \epsilon$.

The proof in [Di Menza and Gallo 2007] relies on the tracking of the exponentially growing eigenmode. One may actually improve slightly the result as this was done in Corollary 15. As a matter of fact, this was the result in Theorem 21 that has motivated us for Corollary 15 . 
We focus now on the nonlinear stability issue when there is linear (spectral) stability, that is, when $\left(d P\left(U_{c}\right) / d c\right)_{\mid c=0}<0$. Concerning the Gross-Pitaevskii nonlinearity $(f(\varrho)=1-\varrho)$, for which we have $\left(d P\left(U_{c}\right) / d c\right)_{\mid c=0}<0$, we quote two papers on this question. The first one is the work of P. Gérard and Z. Zhang [2009] where the stability is shown by inverse scattering, hence in a space of functions sufficiently decaying at infinity. The analysis then relies on the integrability of the one-dimensional GP equation. The other work is by F. Béthuel, P. Gravejat, J.-C. Saut and D. Smets [Béthuel et al. 2008b]. They prove the orbital stability of the kink of the Gross-Pitaevskii equation by showing that the kink is a global minimizer of the energy under the constraint that a variant of the momentum is fixed (recall that the definition of the momentum has to be clarified for an arbitrary function in the energy space), and that the corresponding minimizing sequences are compact (up to space translations and phase factors). In this approach, it is crucial (see [Béthuel et al. 2008a; 2008b]) that $E_{\mathrm{kink}}<\mathfrak{c}_{s} P_{\text {kink }}=\mathfrak{c}_{s} r_{0}^{2} \pi$ in order to prevent the dichotomy case for the minimizing sequences. However, since the energy of the kink is equal to

$$
E_{\text {kink }}=4 \int_{-r_{0}^{2}}^{0} \frac{F\left(r_{0}^{2}+\xi\right)}{\sqrt{-\mathscr{V}_{0}(\xi)}} d \xi=2 \int_{-r_{0}^{2}}^{0} \sqrt{\frac{F\left(r_{0}^{2}+\xi\right)}{r_{0}^{2}+\xi}} d \xi=2 \int_{0}^{r_{0}^{2}} \sqrt{\frac{F(\varrho)}{\varrho}} d \varrho,
$$

whereas its momentum is always equal to $r_{0}^{2} \pi$, it is clear that the condition $E_{\text {kink }}<\mathfrak{c}_{s} P_{\text {kink }}=\mathfrak{c}_{s} r_{0}^{2} \pi$ does not hold in general, as shown in the following example.

Example. For $\kappa \geq 0$, consider

$$
f(\varrho) \equiv 1-\varrho+\kappa(1-\varrho)^{3},
$$

which is smooth and decreases to $-\infty$ as the Gross-Pitaevskii nonlinearity. We have $r_{0}=1, \mathfrak{c}_{s}=\sqrt{2}$, $F(\varrho)=(1-\varrho)^{2} / 2+\kappa(1-\varrho)^{4} / 4$ and

$$
E_{\text {kink }}=2 \int_{0}^{r_{0}^{2}} \sqrt{\frac{F(\varrho)}{\varrho}} d \varrho=2 \int_{0}^{r_{0}^{2}} \sqrt{\frac{2(1-\varrho)^{2}+\kappa(1-\varrho)^{4}}{4 \varrho}} d \varrho>\mathfrak{c}_{s} r_{0}^{2} \pi=\pi \sqrt{2}
$$

for $\kappa$ large (the left-hand side tends to $+\infty$ ), and numerical computations show that it is the case for $\kappa \geq 14$. Furthermore, Lemma 20 gives

$$
\left.\sqrt{F(0)} \frac{d P\left(U_{c}\right)}{d c}\right|_{\mid c=0}=-\frac{8}{3}+\frac{1}{2} \int_{0}^{1} \frac{(\varrho-1)^{2}}{\varrho^{3 / 2}}\left(\sqrt{\frac{F(0)}{F(\varrho)}}-1\right) d \varrho .
$$

Since $F(0) / F(\varrho)=(2+\kappa) /\left(2(\varrho-1)^{2}+\kappa(\varrho-1)^{4}\right)$, it can be easily checked that the right-hand side of (10) is a decreasing function of $\kappa$ tending to

$$
-\frac{8}{3}+\frac{1}{2} \int_{0}^{1} \frac{(\varrho-1)^{2}}{\varrho^{3 / 2}}\left(\frac{1}{(\varrho-1)^{2}}-1\right) d \varrho=-1
$$

when $\kappa \rightarrow+\infty$ (by monotone convergence). In particular, for any $\kappa \geq 0$, we have $\left(d P\left(U_{c}\right) / d c\right)_{\mid c=0}<0$; that is, the kink is always (linearly) stable. The energy-momentum diagram for this type of nonlinearity with $\kappa$ large is as in the right part of Figure 4 (the left part correspond to $\kappa$ smaller). 
In comparison with the constraint minimization approach as in [Béthuel et al. 2008a; 2008b], which allows us to establish a global minimization result, the spectral methods as in [Grillakis et al. 1987; Lin 2002] allow us to put forward locally minimizing properties, which turn out to be useful for the stability analysis in dimension one.

In the stability analysis of the kink, one issue is the definition of the momentum $P$, which was up to now given only for maps in $\mathscr{L}_{\mathrm{hy}}$, that is, for maps that never vanish, but the kink vanishes at the origin. In [Béthuel et al. 2008b], the notion of momentum was extended to the whole energy space $\mathscr{E}$, hence including maps vanishing somewhere, as a quantity defined $\bmod 2 \pi$, and was called "untwisted momentum". This notion will be useful for our stability result.

Lemma 22 [Béthuel et al. 2008b]. If $\psi \in \mathscr{L}$, the limit

$$
\mathfrak{P}(\psi) \equiv \lim _{R \rightarrow+\infty}\left[\int_{-R}^{+R}\left\langle i \psi \mid \partial_{x} \psi\right\rangle d x-r_{0}^{2}(\arg (\psi(+R))-\arg (\psi(-R)))\right]
$$

exists in $\mathbb{R} /\left(2 \pi r_{0}^{2} \mathbb{Z}\right)$. The mapping $\mathfrak{P}: \mathscr{E} \rightarrow \mathbb{R} /\left(2 \pi r_{0}^{2} \mathbb{Z}\right)$ is continuous and, if $\psi \in \mathscr{L}$ satisfies $\inf _{\mathbb{R}}|\psi|>0$ (i.e., $\left.\psi \in \mathscr{L}_{\mathrm{hy}}\right)$, then $\mathfrak{P}(\psi)=P(\psi) \bmod 2 \pi r_{0}^{2}$. Finally, if $\Psi \in \mathscr{C}([0, T)$, $\mathscr{L})$ is a solution to (NLS), then $\mathfrak{P}(\Psi(t))$ does not depend on $t$.

Proof. For the sake of completeness, we recall the proof of [Béthuel et al. 2008b]. Let $\psi \in \mathscr{Z}$ and let us verify the Cauchy criterion. Since $|\psi| \rightarrow r_{0}>0$ at $\pm \infty$, we have a lifting $\psi=A_{ \pm} \mathrm{e}^{i \phi_{ \pm}}$in $\left(-\infty,-R_{0}\right)$ and in $\left(+R_{0},+\infty\right)$ for some $R_{0}$ sufficiently large. For $R^{\prime}>R>R_{0}$, we thus have in $\mathbb{R} /\left(2 \pi r_{0}^{2} \mathbb{Z}\right)$

$$
\begin{gathered}
{\left[\int_{-R^{\prime}}^{+R^{\prime}}\left\langle i \psi \mid \partial_{x} \psi\right\rangle d x-r_{0}^{2}\left(\arg \left(\psi\left(+R^{\prime}\right)\right)-\arg \left(\psi\left(-R^{\prime}\right)\right)\right)\right]} \\
-\left[\int_{-R}^{+R}\left\langle i \psi \mid \partial_{x} \psi\right\rangle d x-r_{0}^{2}(\arg (\psi(+R))-\arg (\psi(-R)))\right] \\
=\int_{R}^{R^{\prime}}\left\langle i \psi \mid \partial_{x} \psi\right\rangle d x+\int_{-R^{\prime}}^{-R}\left\langle i \psi \mid \partial_{x} \psi\right\rangle d x \\
-r_{0}^{2}\left(\arg \left(\psi\left(R^{\prime}\right)\right)-\arg (\psi(R))\right)+r_{0}^{2}\left(\arg \left(\psi\left(-R^{\prime}\right)\right)-\arg (\psi(-R))\right) \\
=\int_{R}^{R^{\prime}} A_{+}^{2} \partial_{x} \phi_{+} d x+\int_{-R^{\prime}}^{-R} A_{-}^{2} \partial_{x} \phi_{-} d x-r_{0}^{2}\left(\phi_{+}\left(R^{\prime}\right)-\phi_{+}(R)\right)+r_{0}^{2}\left(\phi_{-}\left(-R^{\prime}\right)-\phi_{-}(-R)\right) \\
=\int_{R}^{R^{\prime}}\left(A_{+}^{2}-r_{0}^{2}\right) \partial_{x} \phi_{+} d x+\int_{-R^{\prime}}^{-R}\left(A_{-}^{2}-r_{0}^{2}\right) \partial_{x} \phi_{-} d x
\end{gathered}
$$

The absolute value of each term is $\leq K \int_{ \pm x \geq \pm R}\left|\partial_{x} \psi\right|^{2}+\left(|\psi|-r_{0}\right)^{2} d x$ and thus tends to zero if $R \rightarrow+\infty$. Thus, $\mathfrak{P}(\psi)$ is well-defined. The proof of the continuity follows the same lines, and allows us to show that $\mathfrak{P}$ is actually locally Lipschitz continuous. Let $\psi \in \mathscr{L}$. If $\tilde{\psi} \in \mathscr{Z}$ and $d_{\mathscr{L}}(\tilde{\psi}, \psi)$ is small enough, we have $\||\tilde{\psi}|-|\psi|\|_{L^{\infty}}$ as small as we want. In particular, if $R_{0}>0$ is large enough so that $|\psi| \geq 3 r_{0} / 4$ for $|x| \geq R$, we have $|\tilde{\psi}| \geq r_{0} / 2$ for $|x| \geq R_{0}$. As a consequence, writing $\psi=A_{ \pm} \mathrm{e}^{i \phi_{ \pm}}$and 
$\tilde{\psi}=\tilde{A}_{ \pm} \mathrm{e}^{i \tilde{\phi}_{ \pm}}$in $\left(-\infty,-R_{0}\right)$ and in $\left(+R_{0},+\infty\right)$, we have, in $\mathbb{R} /\left(2 \pi r_{0}^{2} \mathbb{Z}\right)$ and for $R>R_{0}$,

$$
\begin{aligned}
& {\left[\int_{-R}^{+R}\left\langle i \psi \mid \partial_{x} \psi\right\rangle d x-r_{0}^{2}(\arg (\psi(+R))-\arg (\psi(-R)))\right]} \\
& \quad-\left[\int_{-R}^{+R}\left\langle i \tilde{\psi} \mid \partial_{x} \tilde{\psi}\right\rangle d x-r_{0}^{2}(\arg (\tilde{\psi}(+R))-\arg (\tilde{\psi}(-R)))\right] \\
& =\int_{-R_{0}}^{+R_{0}}\left\langle i(\psi-\tilde{\psi}) \mid \partial_{x} \psi\right\rangle+\left\langle i \tilde{\psi} \mid \partial_{x}(\psi-\tilde{\psi})\right\rangle d x+\int_{R_{0}}^{R} A_{+}^{2} \partial_{x} \phi_{+}-\tilde{A}_{+}^{2} \partial_{x} \tilde{\phi}_{+} d x-r_{0}^{2}\left(\phi_{+}(+R)-\tilde{\phi}_{+}(+R)\right) \\
& \quad+\int_{-R}^{-R_{0}} A_{-}^{2} \partial_{x} \phi_{-}-\tilde{A}_{-}^{2} \partial_{x} \tilde{\phi}_{-} d x+r_{0}^{2}\left(\phi_{-}(-R)-\tilde{\phi}_{-}(-R)\right) \\
& =\int_{-R_{0}}^{+R_{0}}\left\langle i(\psi-\tilde{\psi}) \mid \partial_{x} \psi\right\rangle+\left\langle i \tilde{\psi} \mid \partial_{x}\left(\psi-\tilde{\psi}_{)}\right)\right\rangle d x+r_{0}^{2}\left(\phi_{+}\left(+R_{0}\right)-\tilde{\phi}_{+}\left(+R_{0}\right)\right)+r_{0}^{2}\left(\phi_{-}\left(-R_{0}\right)-\tilde{\phi}_{-}\left(-R_{0}\right)\right) \\
& \quad+\int_{R_{0}}^{R}\left(A_{+}^{2}-r_{0}^{2}\right) \partial_{x} \phi_{+}-\left(\tilde{A}_{+}^{2}-r_{0}^{2}\right) \partial_{x} \tilde{\phi}_{+} d x+\int_{-R}^{-R_{0}}\left(A_{-}^{2}-r_{0}^{2}\right) \partial_{x} \phi_{-}-\left(\tilde{A}_{-}^{2}-r_{0}^{2}\right) \partial_{x} \tilde{\phi}_{-} d x .
\end{aligned}
$$

We now estimate all the terms. For the last line, we use the Cauchy-Schwarz inequality to get $\left|\int_{R_{0}}^{R}\left(A_{+}^{2}-r_{0}^{2}\right) \partial_{x} \phi_{+} d x\right| \leq K(\psi)\left\|A_{+}-r_{0}\right\|_{L^{2}(\mathbb{R})}\left\|A_{+} \partial_{x} \phi_{+}\right\|_{L^{2}(\mathbb{R})} \leq K(\psi) d_{\mathscr{L}}(\psi, \tilde{\psi})$, and similarly for the other terms. Moreover, using that $(\psi-\tilde{\psi})(x)=(\psi-\tilde{\psi})(0)+\int_{0}^{x} \partial_{x}(\psi-\tilde{\psi})$, we get, by the CauchySchwarz inequality, $\|\psi-\tilde{\psi}\|_{\mathscr{C}^{0}\left(\left[-R_{0},+R_{0}\right]\right)} \leq|(\psi-\tilde{\psi})(0)|+\sqrt{R_{0}}\left\|\partial_{x} \psi-\partial_{x} \tilde{\psi}\right\|_{L^{2}(\mathbb{R})} \leq K\left(R_{0}\right) d_{\mathscr{L}}(\psi, \tilde{\psi})$. Thus, the terms of the second line can be estimated by $K\left(\psi, R_{0}\right) d_{\mathscr{L}}(\psi, \tilde{\psi})$, and those of the first line can also be bounded by $K\left(\psi, R_{0}\right) d_{\mathscr{L}}(\psi, \tilde{\psi})$. Passing to the limit as $R \rightarrow+\infty$ then gives

$$
\left|\mathfrak{P}(\psi)-\mathfrak{P}(\tilde{\psi}) \bmod 2 \pi r_{0}^{2}\right| \leq K\left(\psi, R_{0}\right) d_{\mathscr{L}}(\psi, \tilde{\psi}) .
$$

This completes the proof for the definition of $\mathfrak{P}$. To show that $\mathfrak{P}$ is constant under the (NLS) flow, we use that $\Psi \in \Psi(0)+\mathscr{C}\left([0, T), H^{1}\right)$ and the approximation by smoother solutions (see Proposition 1 in [Béthuel et al. 2008b]).

For the stability of the kink, we can no longer use the Grillakis-Shatah-Strauss theory applied to the hydrodynamical formulation of (NLS), since the kink vanishes at the origin. Therefore, it is natural to consider the Liapounov functional $\mathscr{L}$ introduced in Section 1B2, which becomes, in the stationary case $c=0$,

$$
\mathscr{L}(\psi)=E(\psi)+\frac{M}{2}\left(P(\psi)-P\left(U_{0}\right)\right)^{2} .
$$

Since the momentum $P$ is not well-defined in $\mathscr{E}$, we have to replace it by the untwisted momentum $\mathfrak{P}$, which is defined modulo $2 \pi r_{0}^{2}$. Consequently, it is natural to define the functional in $\mathscr{L}$

$$
\mathscr{K}(\psi) \equiv E(\psi)+2 M r_{0}^{4} \sin ^{2} \frac{\mathfrak{P}(\psi)-r_{0}^{2} \pi}{2 r_{0}^{2}},
$$

which is well-defined and continuous in $\mathscr{E}$ since $\sin ^{2}$ is $\pi$-periodic. In addition, $\mathscr{K}$ is conserved by the (NLS) flow as $E$ and $\mathfrak{P}$. 
Theorem 23. Assume that there exists a kink, that is, a nontrivial stationary $(c=0)$ wave $U_{0}$ which is odd. Assume also that

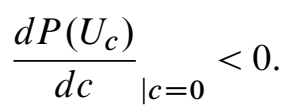

Then, there exists some small $\mu_{*}>0$ such that $U_{0}$ is a local minimizer of $\mathscr{K}$. More precisely, defining

$$
\mathscr{V}_{\mu_{*}} \equiv\left\{\psi \in \mathscr{L}, \inf _{\mathbb{R}}|\psi|<\mu_{*}\right\},
$$

we have, for any $\psi \in \mathcal{V}_{\mu_{*}} \backslash\left\{\mathrm{e}^{i \theta} U_{0}(\cdot-y), \theta \in \mathbb{R}, y \in \mathbb{R}\right\}$,

$$
\mathscr{K}(\psi)>\mathscr{K}\left(U_{0}\right)=E\left(U_{0}\right) .
$$

The crucial point in this result is to prove that the functional $\mathscr{K}(\psi)$ controls the infimum $\inf _{\mathbb{R}}|\psi|$. As we shall see in the proof (Section 6B), the key idea is to study the infimum of the functional $\mathscr{K}$ at fixed $\inf _{\mathbb{R}}|\psi|$ (small), and then to prove (see Proposition 6.2) that, for $\psi \in \mathscr{V}_{\mu_{*}}$, there holds, for some constant $K$ depending only on $f$,

$$
\mathscr{K}(\psi) \geq \mathscr{K}\left(U_{0}\right)+\frac{\left(\inf _{\mathbb{R}}|\psi|\right)^{2}}{K} .
$$

This will be achieved by a fine analysis of some minimizing sequences. From this locally minimizing property of the kink when $\left(d P\left(U_{c}\right) / d c\right)_{\mid c=0}<0$, we infer its orbital stability, provided we can prove some compactness on the minimizing sequences. A main step here is the control on $\inf _{\mathbb{R}}|\psi|$. Our method allows to infer a control on the distance of the solution to (NLS) to the orbit of the kink, but it is much weaker than those obtained by spectral methods in Lemma 7 or Corollary 11 for instance.

Theorem 24. Assume that there exists a kink, that is, an odd nontrivial stationary $(c=0)$ wave $U_{0}$, and

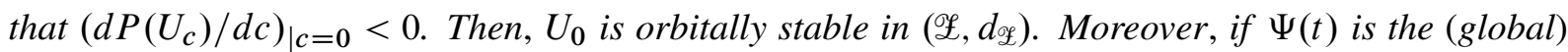
solution to (NLS) with initial datum $\Psi^{\mathrm{in}}$, we have the control

$$
\sup _{t \geq 0} \inf _{\substack{y \in \mathbb{R} \\ \theta \in \mathbb{R}}} d_{\mathscr{L}}\left(\Psi(t), \mathrm{e}^{i \theta} U_{0}(\cdot-y)\right) \leq K \sqrt[8]{\mathscr{K}\left(\Psi^{\mathrm{in}}\right)-E\left(U_{0}\right)} \leq K \sqrt[4]{d_{\mathscr{L}}\left(\Psi^{\mathrm{in}}, U_{0}\right)}
$$

provided that the right-hand side is sufficiently small.

This result settles the nonlinear stability under the condition $\left(d P\left(U_{c}\right) / d c\right)_{\mid c=0}<0$ for a general nonlinearity $f$. In particular, it may be applied to the nonlinearity $f$ given in the example above. It shows that the stability of the kink holds with $\delta=\mathcal{O}\left(\varepsilon^{4}\right)$. We do not claim that the exponent $1 / 8$ is optimal.

For a complete study of the stability of the traveling waves, it would remain to investigate the case of the sonic $\left(c=\mathfrak{c}_{s}\right)$ traveling waves (when they exist). The methods we have developed do not apply directly, and we give in Section 7 some of the difficulties associated with this critical situation.

\section{Decay at infinity (proof of Proposition 2)}

For simplicity, we shall define

$$
\mathscr{V}(\xi) \equiv \mathscr{V}_{\mathfrak{c}_{s}}(\xi)=\mathfrak{c}_{s}^{2} \xi^{2}-4\left(r_{0}^{2}+\xi\right) F\left(r_{0}^{2}+\xi\right) .
$$


We freeze the invariance by translation by imposing $\left|U_{c}\right|$ (hence also $\partial_{x} \phi$ ) even, so that we can use the formulas in [Chiron 2012]. In particular, it suffices to show the asymptotics for $x \rightarrow+\infty$ : the case $x \rightarrow-\infty$ follows by symmetry. We start with the proof of case (iii). Under assumption $\left(\mathscr{A}_{m}\right)$ and since $F^{\prime}=-f$, we infer the Taylor expansion

$$
\begin{aligned}
\mathscr{V}(\xi)= & \mathfrak{c}_{s}^{2} \xi^{2}+4\left(r_{0}^{2}+\xi\right)\left(\frac{1}{2 !} f^{\prime}\left(r_{0}^{2}\right) \xi^{2}+\cdots+\frac{1}{(m+2) !} f^{(m+1)}\left(r_{0}^{2}\right) \xi^{m+2}\right. \\
& \left.\quad+\frac{1}{(m+3) !} f^{(m+2)}\left(r_{0}^{2}\right) \xi^{m+3}+\mathcal{O}\left(\xi^{m+4}\right)\right) \\
= & \frac{4 r_{0}^{2}}{(m+3) !} f^{(m+2)}\left(r_{0}^{2}\right) \xi^{m+3}+\frac{4}{(m+2) !} f^{(m+1)}\left(r_{0}^{2}\right) \xi^{m+3}+\mathcal{O}\left(\xi^{m+4}\right) \\
= & \frac{4}{r_{0}^{2(m+1)}}\left[\frac{r_{0}^{2(m+2)}}{(m+3) !} f^{(m+2)}\left(r_{0}^{2}\right)+(-1)^{m+2} \frac{\mathfrak{c}_{s}^{2}}{4}\right] \xi^{m+3}+\mathcal{O}\left(\xi^{m+4}\right)=\Lambda_{m} \xi^{m+3}+\mathcal{O}\left(\xi^{m+4}\right),
\end{aligned}
$$

since, when $\left(A_{m}\right)$ holds, all the terms $\mathcal{O}\left(\xi^{m+2}\right)$ cancel out. The coefficient $\Lambda_{m}$ is not zero by assumption. Note that the existence of a nontrivial sonic wave, which depends on the global behavior of $\mathcal{V}$, imposes that $\Lambda_{m} \xi^{m+3}<0$ when $\xi$ is small and has the sign of $\xi_{\mathfrak{c}_{s}}$. Therefore, from the formula (following from the Hamiltonian equation $2 \partial_{x}^{2} \eta_{c}+\mathscr{V}^{\prime}\left(\eta_{c}\right)=0$; see [Chiron 2012] for example)

$$
x=-\operatorname{sgn}\left(\xi_{c_{s}}\right) \int_{\xi_{c_{s}}}^{\eta_{c_{s}}(x)} \frac{d \xi}{\sqrt{-\mathscr{V}(\xi)}}
$$

and since we have, as $\eta \rightarrow 0$ (with the sign of $\xi_{c_{s}}$ ),

$$
\begin{aligned}
\int_{\xi_{c s}}^{\eta} \frac{d \xi}{\sqrt{-\mathscr{V}(\xi)}} & =\int_{\xi_{c s}}^{\eta} \frac{d \xi}{\sqrt{-\Lambda_{m} \xi^{m+3}}}+\int_{\xi_{c s}}^{\eta} \frac{\mathscr{V}(\xi)-\Lambda_{m} \xi^{m+3}}{\sqrt{-\mathscr{V}(\xi)} \sqrt{-\Lambda_{m} \xi^{m+3}}\left[\sqrt{-\mathscr{V}(\xi)}+\sqrt{-\Lambda_{m} \xi^{m+3}}\right]} d \xi \\
& =-\frac{2 \operatorname{sgn}\left(\xi_{\mathfrak{c}_{s}}\right)}{m+1}\left(\frac{1}{\sqrt{-\Lambda_{m} \eta^{m+1}}}-\frac{1}{\sqrt{-\Lambda_{m} \xi_{c_{s}}^{m+1}}}\right)+ \begin{cases}\mathcal{O}(1) & \text { if } m=0, \\
\mathcal{O}(|\ln | \eta||) & \text { if } m=1, \\
\mathcal{O}\left(\eta^{-\frac{m-1}{2}}\right) & \text { if } m \geq 2\end{cases}
\end{aligned}
$$

(here, we use that the last integrand is $\mathcal{O}\left(\xi^{-(m+1) / 2}\right)$ as $\xi \rightarrow 0$ ), it follows that, as $x \rightarrow+\infty$,

$$
\eta_{\mathfrak{c}_{s}}(x)=\operatorname{sgn}\left(\xi_{\mathfrak{c}_{s}}\right)\left(\frac{4}{(m+1)^{2}\left|\Lambda_{m}\right|}\right)^{\frac{1}{m+1}} \frac{1}{x^{\frac{2}{m+1}}}+ \begin{cases}\mathcal{O}\left(1 / x^{3}\right) & \text { if } m=0 \\ \mathcal{O}\left(\ln (x) / x^{2}\right) & \text { if } m=1 \\ \mathcal{O}\left(1 / x^{\frac{4}{m+1}}\right) & \text { if } m \geq 2 .\end{cases}
$$

This shows the asymptotics for the modulus, or $\eta_{\mathfrak{c}_{s}}$. The asymptotic expansion for $\partial_{x} \phi_{\mathfrak{c}_{s}}$ is easily deduced from the equation on the phase $2 \partial_{x} \phi_{\mathfrak{c}_{s}}=\mathfrak{c}_{s} \eta_{\mathfrak{c}_{s}} /\left(r_{0}^{2}+\eta_{\mathfrak{c}_{s}}\right)$, and the phase $\phi_{\mathfrak{c}_{s}}$ is then computed by integration, which completes the proof of case (iii).

The proof of (ii) is easier. Indeed, in this case, the function $\mathscr{V}$ has the expansion

$$
\mathscr{V}(\xi)=\mathfrak{c}_{s}^{2} \xi^{2}-4\left(r_{0}^{2}+\xi\right) F\left(r_{0}^{2}+\xi\right)=\mathcal{O}\left(\xi^{3}\right)
$$


hence

$$
\mathscr{V}_{c}(\xi)=\mathscr{V}(\xi)-\left(\mathfrak{c}_{s}^{2}-c^{2}\right) \xi^{2}=-\left(\mathfrak{c}_{s}^{2}-c^{2}\right) \xi^{2}+\mathcal{O}\left(\xi^{3}\right)
$$

As a consequence, the result follows from the expansion, for $\eta \rightarrow 0$,

$$
\begin{aligned}
\int_{\xi_{c}}^{\eta} \frac{d \xi}{\sqrt{-\mathscr{V}(\xi)}} & =\int_{\xi_{c}}^{\eta} \frac{d \xi}{\sqrt{\left(\mathfrak{c}_{s}^{2}-c^{2}\right) \xi^{2}}}+\int_{\xi_{c}}^{\eta} \frac{\mathscr{V}(\xi)}{\sqrt{-\mathscr{V}_{c}(\xi)} \sqrt{\left(\mathfrak{c}_{s}^{2}-c^{2}\right) \xi^{2}}\left[\sqrt{-\mathscr{V}_{c}(\xi)}+\sqrt{\left(\mathfrak{c}_{s}^{2}-c^{2}\right) \xi^{2}}\right]} d \xi \\
& =\operatorname{sgn}\left(\xi_{c}\right) \frac{\ln \left(\eta / \xi_{c}\right)}{\sqrt{\mathfrak{c}_{s}^{2}-c^{2}}}+\int_{\xi_{c}}^{0} \frac{\mathscr{V}(\xi)}{\sqrt{-\mathscr{V}_{c}(\xi)} \sqrt{\left(\mathfrak{c}_{s}^{2}-c^{2}\right) \xi^{2}}\left[\sqrt{-\mathscr{V}_{c}(\xi)}+\sqrt{\left(\mathfrak{c}_{s}^{2}-c^{2}\right) \xi^{2}}\right]} d \xi+O(\eta)
\end{aligned}
$$

since the integrand for the last integral is continuous at $\xi=0$. This yields the desired expansion for the modulus:

$$
\begin{aligned}
\eta_{c}(x) & =\xi_{c} \exp \left(-x \sqrt{\mathfrak{c}_{s}^{2}-c^{2}}-\int_{\xi_{c}}^{0} \frac{\mathscr{V}(\xi)}{\sqrt{-\xi^{2} \mathscr{V}_{c}(\xi)}\left[\sqrt{-\mathscr{V}_{c}(\xi)}+\sqrt{\left(\mathfrak{c}_{s}^{2}-c^{2}\right) \xi^{2}}\right]} d \xi\right)+\mathcal{O}\left[\exp \left(-2 x \sqrt{\mathfrak{c}_{s}^{2}-c^{2}}\right)\right] \\
& =M_{c} \exp \left(-x \sqrt{\mathfrak{c}_{s}^{2}-c^{2}}\right)+\mathcal{O}\left[\exp \left(-2 x \sqrt{\mathfrak{c}_{s}^{2}-c^{2}}\right)\right]
\end{aligned}
$$

with

$$
M_{c} \equiv \xi_{c} \exp \left(-\int_{\xi_{c}}^{0} \frac{\mathscr{V}(\xi)}{\sqrt{-\xi^{2} \mathscr{V}_{c}(\xi)}\left[\sqrt{-\mathscr{V}_{c}(\xi)}+\sqrt{\left(\mathfrak{c}_{s}^{2}-c^{2}\right) \xi^{2}}\right]} d \xi\right) \neq 0
$$

and hence for the phase by similar computations to those above. The proof of case (i) is similar, separating the case $\xi_{0}=-r_{0}^{2}$ of the kink (even solution) from the case $\xi_{0} \neq-r_{0}^{2}$ of the bubble (odd solution), and is omitted.

\section{Stability results deduced from the hydrodynamical formulation of (NLS)}

3A. Proof of Lemma 9. (i) The mapping $\mathcal{M}$ is an homeomorphism. Let $\psi=A \mathrm{e}^{i \phi},\left(\psi_{n}=A_{n} \mathrm{e}^{i \phi_{n}}\right)_{n} \in \mathscr{L}$ such that $\psi_{n} \rightarrow \psi$ for $d_{\text {hy }}$. Then, $A_{n}-A \rightarrow 0$ in $H^{1}, \partial_{x} \phi_{n} \rightarrow \partial_{x} \phi$ in $L^{2}$ and we may assume (possibly adding some multiple of $2 \pi$ to $\left.\phi_{n}\right)$, that $\phi_{n}(0) \rightarrow \phi(0)$. We write, using the embedding $H^{1}(\mathbb{R}) \hookrightarrow L^{\infty}(\mathbb{R})$ for the second-to-last line,

$$
\begin{aligned}
d_{\mathscr{L}} & \left(\psi_{n}, \psi\right) \\
= & \left\|\partial_{x} \psi_{n}-\partial_{x} \psi\right\|_{L^{2}}+\left\|\left|\psi_{n}\right|-|\psi|\right\|_{L^{2}}+\left|\psi_{n}(0)-\psi(0)\right| \\
= & \left\|\mathrm{e}^{i \phi_{n}} \partial_{x} A_{n}+i A_{n} \mathrm{e}^{i \phi_{n}} \partial_{x} \phi_{n}-\mathrm{e}^{i \phi} \partial_{x} A-i A \mathrm{e}^{i \phi} \partial_{x} \phi\right\|_{L^{2}}+\left\|A_{n}-A\right\|_{L^{2}}+\left|A_{n}(0) \mathrm{e}^{i \phi_{n}(0)}-A(0) \mathrm{e}^{i \phi(0)}\right| \\
\leq & \left\|\left(\mathrm{e}^{i \phi_{n}}-\mathrm{e}^{i \phi}\right) \partial_{x} A\right\|_{L^{2}}+\left\|\mathrm{e}^{i \phi}\left(\partial_{x} A_{n}-\partial_{x} A\right)\right\|_{L^{2}}+\left\|\left(A_{n}-A\right) \mathrm{e}^{i \phi_{n}} \partial_{x} \phi_{n}\right\|_{L^{2}}+\left\|A\left(\mathrm{e}^{i \phi_{n}}-\mathrm{e}^{i \phi}\right) \partial_{x} \phi\right\|_{L^{2}} \\
& +\left\|A \mathrm{e}^{i \phi_{n}}\left(\partial_{x} \phi-\partial_{x} \phi_{n}\right)\right\|_{L^{2}}+\left\|A_{n}-A\right\|_{L^{2}}+\left|\left(A_{n}(0)-A(0)\right) \mathrm{e}^{i \phi_{n}(0)}\right|+\left|A(0)\left(\mathrm{e}^{i \phi_{n}(0)}-\mathrm{e}^{i \phi(0)}\right)\right| \\
\leq & \left\|\left(\mathrm{e}^{i \phi_{n}}-\mathrm{e}^{i \phi}\right) \partial_{x} A\right\|_{L^{2}}+K\left\|A_{n}-A\right\|_{H^{1}}+\left\|\left(A_{n}-A\right)\right\|_{H^{1}}\left\|\partial_{x} \phi_{n}\right\|_{L^{2}}+\|A\|_{L^{\infty}}\left\|\left(\mathrm{e}^{i \phi_{n}}-\mathrm{e}^{i \phi}\right) \partial_{x} \phi\right\|_{L^{2}} \\
& +\|A\|_{L^{\infty}}\left\|\partial_{x} \phi-\partial_{x} \phi_{n}\right\|_{L^{2}}+\|A\|_{L^{\infty}}\left|\mathrm{e}^{i \phi_{n}(0)}-\mathrm{e}^{i \phi(0)}\right| \\
= & \left\|\left(\mathrm{e}^{i \phi_{n}}-\mathrm{e}^{i \phi}\right) \partial_{x} A\right\|_{L^{2}}+\|A\|_{L^{\infty}}\left\|\left(\mathrm{e}^{i \phi_{n}}-\mathrm{e}^{i \phi}\right) \partial_{x} \phi\right\|_{L^{2}}+o_{n \rightarrow+\infty}(1),
\end{aligned}
$$


from the convergences we have. Now observe that

$$
\phi_{n}(x)=\phi_{n}(0)+\int_{0}^{x} \partial_{x} \phi_{n}(t) d t \rightarrow \phi(0)+\int_{0}^{x} \partial_{x} \phi(t) d t=\phi(0)
$$

pointwise; hence, by the dominated convergence theorem, $\left\|\left(\mathrm{e}^{i \phi_{n}}-\mathrm{e}^{i \phi}\right) \partial_{x} A\right\|_{L^{2}} \rightarrow 0$, and similarly for the other term. Therefore, $d_{\mathscr{E}}\left(\psi_{n}, \psi\right) \rightarrow 0$ as wished.

Let now $\psi=A \mathrm{e}^{i \phi},\left(\psi_{n}=A_{n} \mathrm{e}^{i \phi_{n}}\right)_{n} \in \mathscr{L}$ such that $\psi_{n} \rightarrow \psi$ for $d_{\mathscr{L}}$. Then, $A_{n}-A=\left|\psi_{n}\right|-|\psi| \rightarrow 0$ in $L^{2}, \partial_{x} \psi_{n} \rightarrow \partial_{x} \psi$ in $L^{2}$ and $\psi_{n}(0) \rightarrow \psi(0)$. Since $|\cdot|$ is 1-Lipschitz continuous, we infer for the modulus

$$
\left\|\partial_{x} A_{n}-\partial_{x} A\right\|_{L^{2}}=\left\|\partial_{x}\left|\psi_{n}\right|-\partial_{x}|\psi|\right\|_{L^{2}} \leq\left\|\partial_{x} \psi_{n}-\partial_{x} \psi\right\|_{L^{2}}
$$

Moreover, $\psi_{n}(0) \rightarrow \psi(0)$ and this implies $\arg \left(\psi_{n}(0) / \psi(0)\right) \rightarrow 0$. Therefore, it suffices to show that $\partial_{x} \phi_{n} \rightarrow \partial_{x} \phi$ in $L^{2}$. We use the formula $A^{2} \partial_{x} \phi=\left\langle i \psi \mid \partial_{x} \psi\right\rangle$, which yields

$$
\begin{aligned}
\partial_{x} \phi_{n}-\partial_{x} \phi & =\frac{\left\langle i \psi_{n} \mid \partial_{x} \psi_{n}\right\rangle}{A_{n}^{2}}-\frac{\left\langle i \psi \mid \partial_{x} \psi\right\rangle}{A^{2}} \\
& =\left\langle i \psi_{n} \mid \partial_{x} \psi_{n}\right\rangle\left(\frac{1}{A_{n}^{2}}-\frac{1}{A^{2}}\right)+\frac{\left\langle i \psi_{n} \mid \partial_{x}\left(\psi-\psi_{n}\right)\right\rangle}{A^{2}}-\frac{\left\langle i\left(\psi-\psi_{n}\right) \mid \partial_{x} \psi\right\rangle}{A^{2}} ;
\end{aligned}
$$

hence

$$
\begin{aligned}
& \left\|\partial_{x} \phi_{n}-\partial_{x} \phi\right\|_{L^{2}} \\
& \quad \leq \frac{\left\|\psi_{n}\right\|_{L^{\infty}}\|A\|_{L^{\infty}}}{\left.\inf _{\mathbb{R}} A^{2}\right)\left(\inf _{\mathbb{R}} A_{n}^{2}\right)}\left\|A_{n}-A\right\|_{L^{2}}+\frac{\left\|\psi_{n}\right\|_{L^{\infty}}}{\inf _{\mathbb{R}} A^{2}}\left\|\partial_{x} \psi-\partial_{x} \psi_{n}\right\|_{L^{2}}+\frac{1}{\inf _{\mathbb{R}} A^{2}}\left\|\left|\psi_{n}-\psi\right| \partial_{x} \psi\right\|_{L^{2}} .
\end{aligned}
$$

The first two terms tend to zero as $n \rightarrow+\infty$. For the last term, we use here again the dominated convergence theorem since $\psi_{n}(x)=\psi_{n}(0)+\int_{0}^{x} \partial_{x} \psi_{n}(t) d t \rightarrow \psi(0)+\int_{0}^{x} \partial_{x} \psi(t) d t=\psi(0)$ pointwise. This concludes the proof of (i).

Proof of (ii). Let us define $\phi_{*}: \mathbb{R} \rightarrow \mathbb{R}$ by $\phi_{*}(x) \equiv \frac{1}{2}(\ln x)^{2} \mathbf{1}_{x \geq 1}$. Then, straightforward computations give $\partial_{x} \phi_{*}(x)=((\ln x) / x) \mathbf{1}_{x \geq 1} \in L^{2}(\mathbb{R})$ and, for $X \geq \mathrm{e}$, by monotonicity of $\partial_{x} \phi_{*}$,

$$
\int_{X}^{2 X}\left(\partial_{x} \phi_{*}\right)^{2} d x \geq X \frac{\ln ^{2}(2 X)}{(2 X)^{2}} \geq \frac{(\ln X)^{2}}{4 X}
$$

We now consider $\phi_{n}: \mathbb{R} \rightarrow \mathbb{R}$ defined by $\phi_{n}(x)=0$ if $x \leq 0$ or $x \geq 3 n \pi, \phi_{n}(x)=x / n$ if $0 \leq x \leq n \pi$, $\phi_{n}(x)=\pi$ if $n \pi \leq x \leq 2 n \pi$ and $\phi_{n}(x)=3 \pi-x / n$ if $2 n \pi \leq x \leq 3 n \pi$. Then, we easily obtain

$$
d_{\text {hy }}\left(\mathrm{e}^{i \phi_{*}}, \mathrm{e}^{i \phi_{*}+i \phi_{n}}\right)=\left\|\partial_{x} \phi_{n}\right\|_{L^{2}}=\sqrt{2 \times \frac{\pi n}{n^{2}}}=\sqrt{\frac{2 \pi}{n}} \rightarrow 0 .
$$

Moreover,

$$
d_{\mathscr{E}}\left(\mathrm{e}^{i \phi_{*}}, \mathrm{e}^{i \phi_{*}+i \phi_{n}}\right)=\left\|\partial_{x} \phi_{*} \mathrm{e}^{i \phi_{*}}-\left(\partial_{x} \phi_{*}+\partial_{x} \phi_{n}\right) \mathrm{e}^{i \phi_{*}+i \phi_{n}}\right\|_{L^{2}} \geq\left\|\partial_{x} \phi_{*}\left(\mathrm{e}^{i \phi_{n}}-1\right)\right\|_{L^{2}}-\left\|\partial_{x} \phi_{n}\right\|_{L^{2}},
$$

and, by our choice of $\phi_{n}$ and using (13),

$$
\left.\left\|\partial_{x} \phi_{*}\left(\mathrm{e}^{i \phi_{n}}-1\right)\right\|_{L^{2}}^{2} \geq \int_{n \pi}^{2 n \pi} 4\left(\partial_{x} \phi_{*}\right)^{2} d x \geq \frac{(\ln X)^{2}}{X} \mid X=n \pi\right) \frac{(\ln n)^{2}}{n \pi} .
$$


Since $(\ln n) / \sqrt{n \pi} \gg \sqrt{2 \pi / n}=d_{\mathrm{hy}}\left(\mathrm{e}^{i \phi_{*}}, \mathrm{e}^{i \phi_{*}+i \phi_{n}}\right)$, it follows that, as wished,

$$
d_{\mathscr{L}}\left(\mathrm{e}^{i \phi_{*}}, \mathrm{e}^{i \phi_{*}+i \phi_{n}}\right) \geq \frac{\ln n}{\sqrt{n \pi}}(1+o(1)) \gg \sqrt{\frac{2 \pi}{n}}=d_{\mathrm{hy}}\left(\mathrm{e}^{i \phi_{*}}, \mathrm{e}^{i \phi_{*}+i \phi_{n}}\right) .
$$

We do not know whether the mapping $\mathcal{M}$ is locally Lipschitz, but it is probably not.

3B. Proof of Lemma 10. Note first that, since $U_{*}$ does not vanish, if $\delta$ is sufficiently small and $d_{\mathscr{E}}\left(\psi, U_{*}\right) \leq \delta$, then $\left\||\psi|-\left|U_{*}\right|\right\|_{L^{\infty}} \leq(1 / 2) \inf _{\mathbb{R}}\left|U_{*}\right|$; hence $|\psi| \geq(1 / 2) \inf _{\mathbb{R}}\left|U_{*}\right|>0$ in $\mathbb{R}$; thus $\psi$ does not vanish, may be lifted to $\psi=A \exp (i \phi)$, and we may further assume $\phi(0)-\phi_{*}(0) \in(-\pi,+\pi]$. In (11), we can easily check that the terms leading to the " $o(1)$ " are indeed controlled by $K(\psi) d_{\text {hy }}\left(\psi_{n}, \psi\right)$. In other words, we have

$$
d_{\mathscr{L}}\left(\psi, U_{*}\right) \leq\left\|\left(\mathrm{e}^{i \phi}-\mathrm{e}^{i \phi_{*}}\right) \partial_{x} A_{*}\right\|_{L^{2}}+\left\|A_{*}\right\|_{L^{\infty}}\left\|\left(\mathrm{e}^{i \phi}-\mathrm{e}^{i \phi_{*}}\right) \partial_{x} \phi_{*}\right\|_{L^{2}}+K\left(U_{*}\right) d_{\text {hy }}\left(\psi, U_{*}\right),
$$

provided $d_{\mathrm{hy}}\left(\psi, U_{*}\right)$ is small enough. In order to bound the two remaining terms, we write, for $x \in \mathbb{R}$,

$$
\phi(x)-\phi_{*}(x)=\phi(0)-\phi_{*}(0)+\int_{0}^{x} \partial_{x} \phi(y)-\partial_{x} \phi_{*}(y) d y,
$$

which implies, using that $\mathbb{R} \ni \theta \mapsto \mathrm{e}^{i \theta}$ is 1-Lipschitz and the Cauchy-Schwarz inequality,

$$
\left|1-\mathrm{e}^{i\left(\phi_{*}(x)-\phi(x)\right)}\right| \leq\left|\phi(0)-\phi_{*}(0)\right|+\sqrt{|x|}\left\|u-u_{*}\right\|_{L^{2}} .
$$

Consequently,

$$
\left\|\left(\mathrm{e}^{i \phi}-\mathrm{e}^{i \phi_{*}}\right) \partial_{x} A_{*}\right\|_{L^{2}} \leq\left|\phi(0)-\phi_{*}(0)\right|\left\|\partial_{x} A_{*}\right\|_{L^{2}}+\left\|u-u_{*}\right\|_{L^{2}}\left\|\sqrt{|x|} \partial_{x} A_{*}\right\|_{L^{2}}
$$

and

$$
\left\|\left(\mathrm{e}^{i \phi}-\mathrm{e}^{i \phi_{*}}\right) \partial_{x} \phi_{*}\right\|_{L^{2}} \leq\left|\phi(0)-\phi_{*}(0)\right|\left\|\partial_{x} \phi_{*}\right\|_{L^{2}}+\left\|u-u_{*}\right\|_{L^{2}}\left\|\sqrt{|x|} \partial_{x} \phi_{*}\right\|_{L^{2}} .
$$

Both terms are $\leq K\left(U_{*}\right) d_{\text {hy }}\left(\psi, U_{*}\right)$. Indeed, $U_{*} \in \mathscr{L}$ is a traveling wave; hence $A_{*}, \partial_{x} A_{*}, \partial_{x} \phi_{*}$ are bounded functions which decay at infinity exponentially if $0 \leq c<\mathfrak{c}_{s}$ (cf. part (i) or (ii) of Proposition 2). If $c=\mathfrak{c}_{s}$, since assumption $\left(\mathscr{A}_{0}\right)$ is satisfied, we invoke Proposition 2(iii), which ensures that $\partial_{x} \phi_{*}$ and $\partial_{x} A_{*}$ decay at the rate $O\left(|x|^{-2}\right)\left(\partial_{x} A_{*}\right.$ decays faster actually). Therefore, $\sqrt{|x|} \partial_{x} \phi_{*} \in L^{2}$. Gathering these estimates provides

$$
d_{\mathscr{L}}\left(\psi, U_{*}\right) \leq K\left(U_{*}\right) d_{\mathrm{hy}}\left(\psi, U_{*}\right) .
$$

On the other hand, from (12) and the estimate $\left\|A-A_{*}\right\|_{H^{1}} \leq d_{\mathscr{L}}\left(\psi, U_{*}\right)$ (see the proof of (i)), we infer

$$
d_{\text {hy }}\left(\psi, U_{*}\right) \leq K\left(U_{*}\right) d_{\mathscr{L}}\left(\psi, U_{*}\right)+\frac{1}{\inf _{\mathbb{R}} A^{2}}\left\|\left|\psi-U_{*}\right| \partial_{x} U_{*}\right\|_{L^{2}} .
$$

Using here again the estimate $\left|\psi(x)-U_{*}(x)\right| \leq\left|\phi(0)-\phi_{*}(0)\right|+\sqrt{|x|}\left\|\partial_{x} \psi-U_{*}\right\|_{L^{2}}$, we deduce

$$
d_{\text {hy }}\left(\psi, U_{*}\right) \leq K\left(U_{*}\right) d_{\mathscr{L}}\left(\psi, U_{*}\right) .
$$

The proof is complete. 
3C. Two remarks on the proof of Theorem 5. We would like to point out two minor points concerning the proof of Theorem 5 by Z. Lin. We recall that the proof of [Lin 2002] relies on the Grillakis-ShatahStrauss theory [Grillakis et al. 1987] once we have written (NLS) under the hydrodynamical form (2), defining $\psi=A \mathrm{e}^{i \phi},(\rho, u) \equiv\left(|\psi|^{2}=A^{2}, \partial_{x} \phi\right)$ :

$$
\left\{\begin{array}{l}
\partial_{t} \rho+2 \partial_{x}(\rho u)=0, \\
\partial_{t} u+2 u \partial_{x} u-\partial_{x}(f(\rho))-\partial_{x}\left(\frac{\partial_{x}^{2}(\sqrt{\rho})}{\sqrt{\rho}}\right)=0,
\end{array}\right.
$$

or, more precisely, with $\eta \equiv \rho-r_{0}^{2}=|\psi|^{2}-r_{0}^{2}$ and denoting by $\delta E / \delta \eta, \delta E / \delta u$ the variational derivatives,

$$
\frac{\partial}{\partial t}\left(\begin{array}{l}
\eta \\
u
\end{array}\right)=J\left(\begin{array}{l}
\frac{\delta E}{\delta \eta} \\
\frac{\delta E}{\delta u}
\end{array}\right), \quad J \equiv\left(\begin{array}{cc}
0 & \partial_{x} \\
\partial_{x} & 0
\end{array}\right)
$$

We first remark that the scalar product in the Hilbert space $X=H^{1} \times L^{2}$ can not be $((\eta, u),(\tilde{\eta}, \tilde{u}))_{H^{1} \times L^{2}}=$ $\int_{\mathbb{R}} \eta \tilde{\eta}+u \tilde{u} d x$ as used in [Lin 2002], but the natural one is $((\eta, u),(\tilde{\eta}, \tilde{u}))_{H^{1} \times L^{2}}=\int_{\mathbb{R}} \eta \tilde{\eta}+\partial_{x} \eta \partial_{x} \tilde{\eta}+u \tilde{u} d x$. This requires us to make some minor changes in the proof, especially not to identify $\left(H^{1}\right)^{*}$ with $H^{1}$. For instance, a linear mapping $B$ is associated with the momentum through the formula

$$
P_{\mathrm{hy}}(\eta, u) \equiv \int_{\mathbb{R}} \eta u d x=\frac{1}{2}(B(\eta, u),(\eta, u))_{H^{1} \times L^{2}} \quad \text { with } B \equiv\left(\begin{array}{ll}
0 & 1 \\
1 & 0
\end{array}\right)
$$

for the (nonhilbertian) scalar product $((\eta, u),(\tilde{\eta}, \tilde{u}))_{H^{1} \times L^{2}}=\int_{\mathbb{R}} \eta \tilde{\eta}+u \tilde{u} d x$. The correct definition is actually

$$
P_{\text {hy }}(\eta, u)=\int_{\mathbb{R}} \eta u d x=\frac{1}{2}\langle B(\eta, u),(\eta, u)\rangle_{X^{*}, X} \quad \text { with } B \equiv\left(\begin{array}{cc}
0 & \iota^{*} \\
\iota & 0
\end{array}\right),
$$

where $\iota: H^{1} \hookrightarrow L^{2}$ is the canonical injection. As already mentioned in Section 1B, the two points in the proof of [Lin 2002] that have been completed in [Gallo 2004] are that: Lin uses a local in time existence for the hydrodynamical system (15) in $H^{1} \times L^{2}$, and not only in $\left\{\rho \in L^{\infty}, \partial_{x} \rho \in L^{2}\right\} \times L^{2}$; and that the energy and the momentum are indeed conserved for the local solution if the initial datum does not vanish.

The second point is that, in the proof of stability (Theorem 3.5 in [Grillakis et al. 1987]), it is used that, if $U \in X$ and $\left(\cup_{n}\right)_{n \in \mathbb{N}} \in X$ is a sequence such that $E\left(U_{n}\right) \rightarrow E(U)$ and $P_{\text {hy }}\left(U_{n}\right) \rightarrow P_{\text {hy }}(U)$, then there exists a sequence $\left(\tilde{U}_{n}\right)_{n \in \mathbb{N}} \in X$ such that $U_{n}-\tilde{U}_{n} \rightarrow 0$ in $X, E\left(\tilde{u}_{n}\right) \rightarrow E(U)$ and $P_{\text {hy }}\left(\tilde{U}_{n}\right)=P_{\text {hy }}(U)$. In the context of bound states, the existence of such a sequence $\left(\tilde{U}_{n}\right)_{n \in \mathbb{N}} \in X$ follows by simple scaling in space, since then the momentum or charge is simply $\int_{\mathbb{R}^{d}} U_{n}^{2} d x$. However, for the one-dimensional traveling waves for (NLS), the momentum $P$ is scaling invariant. We do not know if the existence of such a sequence holds in a general framework, but, for the problem we are studying, we can rely on the following lemma, which is an adaptation of Lemma 6 in [Béthuel et al. 2008a] (see also lemma in [Béthuel et al. 2008b]). 
Lemma 3.1. There exist $\mathfrak{p}_{0}>0$ and $K>0$, depending only on $f$, such that, for any $\mathfrak{p} \in\left(-\mathfrak{p}_{0},+\mathfrak{p}_{0}\right)$ and $\mu \in \mathbb{R}$ with $|\mu| \leq|\mathfrak{p}|$, there exists $w=a \mathrm{e}^{i \varphi} \in H^{1}([0,1 /(2|\mathfrak{p}|)), \mathbb{C})$ verifying

$$
\begin{gathered}
w(0)=w\left(\frac{1}{2|\mathfrak{p}|}\right), \quad|w(0)|=r_{0}+\mu, \quad \int_{0}^{1 /(2|\mathfrak{p}|)}\left(a^{2}-r_{0}^{2}\right) \partial_{x} \varphi d x=\mathfrak{p}, \\
\int_{0}^{1 /(2|\mathfrak{p}|)}\left|\partial_{x} w\right|^{2}+F\left(|w|^{2}\right) d x \leq K|\mathfrak{p}| .
\end{gathered}
$$

Proof. If $\mathfrak{p}=0$, we simply take $w=r_{0}$. We then assume $0<\mathfrak{p}<\mathfrak{p}_{0}$, since the case $-\mathfrak{p}_{0}<\mathfrak{p}<0$ will follow by complex conjugation. We then define, for some small $\delta$ to be determined later,

$$
w(x) \equiv \sqrt{r_{0}^{2}-\delta+2 \mathfrak{p}(1-|8 \mathfrak{p} x-1|)_{+}} \exp \left[i(1-|4 \mathfrak{p} x-1|)_{+}\right]=a \mathrm{e}^{i \varphi} .
$$

It is clear that $w \in H^{1}([0,1 /(2 \mathfrak{p})], \mathbb{C})$ and that $w(0)=w(1 /(2 \mathfrak{p}))=\sqrt{r_{0}^{2}-\delta}$; thus $|w(0)|=r_{0}+\mu$ provided we choose $\delta=-\mu^{2}-2 r_{0} \mu=\mathcal{O}(|\mu|)$. Moreover, since the phase $\varphi$ has compact support $[0,1 /(2 \mathfrak{p})]$,

$$
\begin{aligned}
\int_{0}^{1 /(2 \mathfrak{p})}\left(a^{2}-r_{0}^{2}\right) \partial_{x} \varphi d x & =\int_{0}^{1 /(2 \mathfrak{p})}\{-\delta+2 \mathfrak{p}(1-|8 \mathfrak{p} x-1|)+\} \partial_{x}(1-|4 \mathfrak{p} x-1|)_{+} d x \\
& =2 \mathfrak{p} \int_{0}^{1 /(2 \mathfrak{p})}(1-|8 \mathfrak{p} x-1|)_{+} \partial_{x}(1-|4 \mathfrak{p} x-1|)_{+} d x \\
& =2 \mathfrak{p} \int_{0}^{1 /(2 \mathfrak{p})}(1-|8 \mathfrak{p} x-1|)_{+} \partial_{x}(1-|4 \mathfrak{p} x-1|)_{+} d x
\end{aligned}
$$

For the last integral, the first factor is equal to 0 if $x \geq 1 /(4 \mathfrak{p})$ and the second factor is equal to $4 \mathfrak{p}$ when $x \leq 1 /(4 \mathfrak{p})$. Hence, direct computation gives

$$
\int_{0}^{1 /(2 \mathfrak{p})}\left(a^{2}-r_{0}^{2}\right) \partial_{x} \varphi d x=2 \mathfrak{p} \int_{0}^{1 /(4 \mathfrak{p})}(1-|8 \mathfrak{p} x-1|)_{+} \times 4 \mathfrak{p} d x=\mathfrak{p} .
$$

For the energy part, notice first that

$$
\left|a^{2}-r_{0}^{2}\right|=\left|-\delta+2 \mathfrak{p}(1-|8 \mathfrak{p} x-1|)_{+}\right| \leq|\delta|+2 \mathfrak{p}_{0}
$$

is as small as we want if $|\delta|$ and $\mathfrak{p}_{0}$ are chosen sufficiently small. Therefore,

$$
F\left(|w|^{2}\right) \leq K\left(a^{2}-r_{0}^{2}\right)^{2} .
$$

By simple computations, we have

$$
\begin{aligned}
& \int_{0}^{1 /(2 \mathfrak{p})}\left|\partial_{x} w\right|^{2}+F\left(|w|^{2}\right) d x \\
& \quad \leq K \int_{0}^{1 /(2 \mathfrak{p})} \mathfrak{p}^{2}\left|\partial_{x}(1-|8 \mathfrak{p} x-1|)_{+}\right|^{2}+\left|\partial_{x}(1-|4 \mathfrak{p} x-1|)_{+}\right|^{2}+\left(-\delta+2 \mathfrak{p}(1-|8 \mathfrak{p} x-1|)_{+}\right)^{2} d x \\
& \quad \leq K \mathfrak{p}^{3}+K \mathfrak{p}+K \frac{\delta^{2}+\mathfrak{p}^{2}}{\mathfrak{p}} \leq K \mathfrak{p}
\end{aligned}
$$

since $\delta=\mathscr{O}(|\mu|)=\mathscr{O}(\mathfrak{p})$, which concludes the proof. 
We then consider a sequence $u_{n}=\left(\eta_{n}, u_{n}\right) \in X=H^{1} \times L^{2}$ and show the existence of the desired sequence $\tilde{u}_{n}=\left(\tilde{\eta}_{n}, \tilde{u}_{n}\right) \in X$. We recall that $U_{n}$ (respectively, $\mathscr{U}$ ) is associated with a mapping $\psi_{n} \in \mathscr{L}$ (respectively, $U_{*}$ ) that does not vanish. We have $P_{\text {hy }}\left(U_{n}\right)=P\left(\psi_{n}\right) \rightarrow P\left(U_{*}\right)$; thus, for $n$ large enough, $\left|P\left(\psi_{n}\right)-P\left(U_{*}\right)\right| \leq \mathfrak{p}_{0}$. For $n$ fixed, we now pick $R_{n}>0$ large enough so that

$$
\int_{R_{n}}^{+\infty}\left|\partial_{x} \psi_{n}\right|^{2}+\left(\left|\psi_{n}\right|-r_{0}\right)^{2} d x \leq\left[P\left(\psi_{n}\right)-P\left(U_{*}\right)\right]^{2} .
$$

In particular, by the Sobolev embedding,

$$
|| \psi_{n}\left|\left(R_{n}\right)-r_{0}\right| \leq\left\|\left|\psi_{n}\right|-r_{0}\right\|_{L^{\infty}\left(\left[R_{n},+\infty\right)\right)} \leq \sqrt{\int_{R_{n}}^{+\infty}\left|\partial_{x}\right| \psi_{n}||^{2}+\left(\left|\psi_{n}\right|-r_{0}\right)^{2} d x} \leq\left|P\left(\psi_{n}\right)-P\left(U_{*}\right)\right| .
$$

We are now in position to apply (for $n$ large) Lemma 3.1 with (p, $\mu)=\left(P\left(U_{*}\right)-P\left(\psi_{n}\right),\left|\psi_{n}\right|\left(R_{n}\right)-r_{0}\right)$. This provides the mapping $w_{n} \in H^{1}([0,1 /(2|\mathfrak{p}|)), \mathbb{C})$. Since $\left|\psi_{n}\right|\left(R_{n}\right)-r_{0} \rightarrow 0$, for $n$ large enough, there exists $\theta_{n} \in \mathbb{R}$ such that $\psi_{n}\left(R_{n}\right)=\mathrm{e}^{i \theta_{n}}\left|\psi_{n}\right|\left(R_{n}\right)=\mathrm{e}^{i \theta_{n}}\left(r_{0}+\mu\right)=\mathrm{e}^{i \theta_{n}} w_{n}(0)$. We then consider the mapping $\tilde{\psi}_{n} \in \mathscr{L}$ defined by

$$
\tilde{\psi}_{n}(x) \equiv \begin{cases}\psi_{n}(x) & \text { if } x \leq R_{n}, \\ \mathrm{e}^{i \theta_{n}} w_{n}\left(x-R_{n}\right) & \text { if } R_{n} \leq x \leq R_{n}+\frac{1}{2\left|P\left(\psi_{n}\right)-P\left(U_{*}\right)\right|}, \\ \psi_{n}\left(x-\frac{1}{2\left|P\left(\psi_{n}\right)-P\left(U_{*}\right)\right|}\right) & \text { if } x \geq R_{n}+\frac{1}{2\left|P\left(\psi_{n}\right)-P\left(U_{*}\right)\right|} .\end{cases}
$$

From the construction of $w_{n}$ and the phase factor $\theta_{n}, \tilde{\psi}_{n}$ is well-defined and continuous. It is clear that

$$
P\left(\tilde{\psi}_{n}\right)=P\left(\psi_{n}\right)+\int_{0}^{1 /(2 \mathfrak{p})}\left(a_{n}^{2}-r_{0}^{2}\right) \partial_{x} \varphi_{n} d x=P\left(\psi_{n}\right)+\mathfrak{p}=P\left(U_{*}\right)
$$

for every (large) $n$, and that

$$
\begin{aligned}
E\left(\tilde{\psi}_{n}\right) & =E\left(\psi_{n}\right)+\int_{0}^{1 /(2 \mathfrak{p})}\left|\partial_{x} w\right|^{2}+F\left(|w|^{2}\right) d x \\
& =E\left(U_{*}\right)+o(1)+\mathcal{O}(|\mathfrak{p}|)=E\left(U_{*}\right)+o(1)+\mathcal{O}\left(\left|P\left(U_{*}\right)-P\left(\psi_{n}\right)\right|\right)
\end{aligned}
$$

converges to $P\left(U_{*}\right)$ as $n \rightarrow+\infty$. Denoting by $\tilde{\vartheta}_{n} \in X$ the hydrodynamical expression of $\tilde{\psi}_{n}$, it remains to show that $u_{n}-\tilde{U}_{n} \rightarrow 0$ in $X=H^{1} \times L^{2}$. We thus compute, with the definition of $\tilde{\psi}_{n}$,

$$
\begin{aligned}
\left\|u_{n}-\tilde{u}_{n}\right\|_{X}^{2} & =\int_{R_{n}}^{+\infty}\left|\partial_{x}\right| \psi_{n}\left|-\partial_{x}\right| \tilde{\psi}_{n}||^{2}+\left(\left|\psi_{n}\right|-\left|\tilde{\psi}_{n}\right|\right)^{2}+\left(u_{n}-\tilde{u}_{n}\right)^{2} d x \\
& \leq 2 \int_{R_{n}}^{+\infty}\left|\partial_{x}\right| \psi_{n}||^{2}+\left|\partial_{x}\right| \tilde{\psi}_{n}||^{2}+\left(\left|\psi_{n}\right|-r_{0}\right)^{2}+\left(\left|\tilde{\psi}_{n}\right|-r_{0}\right)^{2}+u_{n}^{2}+\tilde{u}_{n}^{2} d x \\
& \leq 4 K \int_{R_{n}}^{+\infty}\left|\partial_{x} \psi_{n}\right|^{2}+\left(\left|\psi_{n}\right|-r_{0}\right)^{2} d x+2 \int_{0}^{1 /\left(2\left|P\left(U_{*}\right)-P\left(\psi_{n}\right)\right|\right)}\left|\partial_{x} w_{n}\right|^{2}+\left(\left|w_{n}\right|^{2}-r_{0}^{2}\right)^{2} d x \\
& \leq 4 K\left[P\left(\psi_{n}\right)-P\left(U_{*}\right)\right]^{2}+K\left|P\left(\psi_{n}\right)-P\left(U_{*}\right)\right| \rightarrow 0 .
\end{aligned}
$$


For the second-to-last inequality, we have used that, for $|x| \geq R_{n}, \psi_{n}$ has modulus uniformly close to $r_{0}$; hence $\left|\partial_{x}\right| \psi_{n}||^{2}+u_{n}^{2} \leq K\left|\partial_{x} \psi_{n}\right|^{2}$. Note that the construction still holds for the energy distance, the computations being similar.

3D. Proof of Lemma 7. Proof of estimate (3). Instead of concluding the stability proof as in [Grillakis et al. 1987], we can notice that we have actually the bound

$$
E_{\mathrm{hy}}(u)-E_{\mathrm{hy}}\left(u_{c_{*}}\right) \geq \frac{1}{K} \inf _{y \in \mathbb{R}}\left\|u-u_{c_{*}}(\cdot-y)\right\|^{2}
$$

as soon as $P\left(U_{c_{*}}\right)=P_{\text {hy }}\left(U_{c_{*}}\right)=P_{\text {hy }}(\mathcal{U})$ and $\mathcal{U} \in \mathcal{O}_{\varepsilon} \equiv\left\{\mathscr{V} \in X\right.$, $\left.\inf _{y \in \mathbb{R}}\left\|\mathscr{V}-\mathcal{U}_{c_{*}}(\cdot-y)\right\|_{X}<\varepsilon\right\}$ for some small $\varepsilon$. If $\Psi^{\text {in }}$ does not have momentum equal to $P_{\text {hy }}\left(U_{c_{*}}\right)$, we use Lemma 3.1 to infer that there exists $\tilde{\Psi}(t)$, with momentum equal to $P_{\mathrm{hy}}\left(u_{c_{*}}\right)=P\left(U_{c_{*}}\right)$, and such that $E(\tilde{\Psi}(t))-E(\Psi(t))=$ $\mathcal{O}\left(\left|P(\Psi(t))-P\left(U_{c_{*}}\right)\right|\right)$ and $d_{\text {hy }}(\Psi(t), \tilde{\Psi}(t)) \leq \mathcal{O}\left(\sqrt{\left|P(\Psi(t))-P\left(U_{c_{*}}\right)\right|}\right)$. Therefore, for $t \geq 0$, denoting by $\Psi_{\text {hy }}(t) \in X$ and $\tilde{\Psi}_{\text {hy }}(t) \in X$ the hydrodynamical variables for $\Psi$ and $\tilde{\Psi}(t)$,

$$
\begin{aligned}
\inf _{y \in \mathbb{R}}\left\|\Psi_{\text {hy }}(t)-u_{c_{*}}(\cdot-y)\right\| & \leq \inf _{y \in \mathbb{R}}\left[\left\|\tilde{\Psi}_{\text {hy }}(t)-u_{c_{*}}(\cdot-y)\right\|+\left\|\Psi_{\text {hy }}(t)-\tilde{\Psi}_{\text {hy }}(t)\right\|\right] \\
& \leq \sqrt{K} \sqrt{E(\tilde{\Psi}(t))-E\left(U_{c_{*}}\right)}+O\left(\sqrt{\left|P(\Psi(t))-P\left(U_{c_{*}}\right)\right|}\right) \\
& \leq K\left[\sqrt{\left|E(\Psi(t))-E\left(U_{c_{*}}\right)\right|+\left|P\left(\Psi^{\text {in }}\right)-P\left(U_{c_{*}}\right)\right|}+\sqrt{\left|P\left(\Psi^{\text {in }}\right)-P\left(U_{c_{*}}\right)\right|}\right],
\end{aligned}
$$

which yields (3).

The above estimate is optimal when $P\left(\Psi^{\mathrm{in}}\right)=P\left(U_{c_{*}}\right)$ since $U_{c_{*}}$ is a critical point of the action $E-c_{*} P$. This bound shows that, in the definition of stability, one has to take $\delta=O\left(\varepsilon^{2}\right)$ in general. The estimate (3) shows that one can actually take $\delta=\mathcal{O}(\varepsilon)$.

Proof of estimate (4). The point is to compare $\Psi(t)$ to $U_{c}$ with $c \simeq c_{*}$ such that $P\left(U_{c}\right)=P\left(\Psi^{\text {in }}\right)$ instead of comparing to $U_{c_{*}}$. In other words, we replace $\tilde{\Psi}(t)$ by $U_{c}$. Note first that, since $(d P / d c)_{\mid c=c_{*}}<0$, there exists, by the implicit function theorem, such a $c \simeq c_{*}$. We then proceed as follows. Let $\Psi^{\text {in }} \in \mathscr{L}$ be close to $U_{c_{*}}$. Then, there exists $c=c\left(\Psi^{\mathrm{in}}\right) \simeq c_{*}$ such that $P\left(U_{c}\right)=P\left(\Psi^{\mathrm{in}}\right)$. Moreover, since $(d P / d c)_{\mid c=c_{*}} \neq 0$, it follows

$$
\begin{aligned}
\left\|u_{c}-u_{c_{*}}\right\| & \leq K\left|c-c_{*}\right| \leq K\left|P\left(U_{c}\right)-P\left(U_{c_{*}}\right)\right|=K\left|P\left(\Psi^{\mathrm{in}}\right)-P\left(U_{c_{*}}\right)\right| \\
& \leq K\left\|\Psi_{\text {hy }}^{\text {in }}-u_{c_{*}}\right\| \leq K d_{\text {hy }}\left(\Psi^{\text {in }}, U_{c_{*}}\right) .
\end{aligned}
$$

From (16), we have

$$
E_{\text {hy }}(u)-E_{\text {hy }}\left(u_{c}\right) \geq \frac{1}{K} \inf _{y \in \mathbb{R}}\left\|u-u_{c}(\cdot-y)\right\|^{2}
$$

as soon as $P_{\text {hy }}(u)=P_{\text {hy }}\left(u_{c}\right)$. The fact that the constant $K$ can be taken to be uniform with respect to $c$ for $c$ close to $c_{*}$ comes directly from the proof in [Grillakis et al. 1987]. Therefore, for $t \geq 0$,

$$
\begin{aligned}
\inf _{y \in \mathbb{R}}\left\|\Psi_{\text {hy }}(t)-u_{c_{*}}(\cdot-y)\right\| & \leq \inf _{y \in \mathbb{R}}\left[\left\|\Psi_{\text {hy }}(t)-u_{c}(\cdot-y)\right\|+\left\|u_{c}(\cdot-y)-u_{c_{*}}(\cdot-y)\right\|\right] \\
& \leq \sqrt{K} \sqrt{E(\Psi(t))-E\left(U_{c}\right)}+O\left(\left|P\left(\Psi^{\mathrm{in}}\right)-P\left(U_{c_{*}}\right)\right|\right) .
\end{aligned}
$$


Using that $P(\Psi(t))=P_{\text {hy }}\left(\Psi_{\text {hy }}(t)\right)=P_{\text {hy }}\left(U_{c}\right)$ and that $U_{c}$ is a critical point of the action $E_{\text {hy }}-c P_{\text {hy }}$, we infer $E(\Psi(t))-E\left(U_{c}\right)=\left[E_{\mathrm{hy}}-c P_{\mathrm{hy}}\right]\left(\Psi_{\mathrm{hy}}^{\mathrm{in}}\right)-\left[E_{\mathrm{hy}}-c P_{\mathrm{hy}}\right]\left(\cup_{c}\right)=\mathcal{O}\left(\left\|\Psi_{\mathrm{hy}}^{\text {in }}-\cup_{c}\right\|^{2}\right)$. Consequently,

$$
\begin{aligned}
\inf _{y \in \mathbb{R}}\left\|\Psi_{\text {hy }}(t)-\vartheta_{c_{*}}(\cdot-y)\right\| & \leq K\left(\left\|\Psi_{\text {hy }}^{\text {in }}-\vartheta_{c}\right\|+\left\|\Psi_{\text {hy }}^{\text {in }}-\vartheta_{c_{*}}\right\|\right) \\
& \leq K d_{\text {hy }}\left(\Psi^{\text {in }}, U_{c_{*}}\right)+K\left\|u_{c}-\vartheta_{c_{*}}\right\| \leq K d_{\text {hy }}\left(\Psi^{\text {in }}, U_{c_{*}}\right),
\end{aligned}
$$

by (17). This gives (4).

\section{Instability result for cusps: Proof of Theorem 16}

In this section, we set $\mathscr{F}_{c} \equiv E_{\mathrm{hy}}-c P_{\text {hy }}$ and we assume

$$
-\left.\frac{d^{2} \mathscr{F}_{c}\left(U_{c}\right)}{d c^{2}}\right|_{c=c_{*}}=\left.\frac{d P\left(U_{c}\right)}{d c}\right|_{\mid c=c_{*}}=0 \quad \text { and } \quad 0 \neq \ddot{P}_{*} \equiv{\frac{d^{2} P\left(U_{c}\right)}{d c^{2}}}_{\mid c=c_{*}}=-\frac{d^{3} \mathscr{F}_{c}\left(U_{c}\right)}{d c^{3}}{ }_{\mid c=c_{*}} .
$$

The approach is reminiscent of the proof of [Maeda 2012]. Several modifications are necessary since, for the skew-adjoint operator $J=\partial_{x}$, we can not find the required Hilbert space $Y$. More degenerate cases can probably be considered as in [Maeda 2012].

We shall denote by $\llbracket: X \rightarrow X^{*}$ and $\rrbracket_{H^{1}}: H^{1} \rightarrow\left(H^{1}\right)^{*}$ the Riesz isomorphisms and define $u=$ $(\eta, u)^{t} \in X=H^{1}(\mathbb{R}, \mathbb{R}) \times L^{2}(\mathbb{R}, \mathbb{R})$ and $H \equiv L^{2}(\mathbb{R}, \mathbb{R}) \times L^{2}(\mathbb{R}, \mathbb{R})$, endowed with its canonical scalar product. They are the corresponding Hilbert spaces needed in [ibid.]. We consider the symmetric matrix

$$
\mathbb{B} \equiv\left(\begin{array}{ll}
0 & 1 \\
1 & 0
\end{array}\right)
$$

which is such that $\mathbb{B}^{2}=\operatorname{Id}_{2}$ and $2 P_{\text {hy }}(U)=(\mathbb{B} U, U)_{H}$.

Our assumption $\left(d P\left(U_{c}\right) / d c\right)_{\mid c=c_{*}}=0 \neq\left(d^{2} P\left(U_{c}\right) / d c^{2}\right)_{\mid c=c_{*}}$ will simplify a little the computations in [Maeda 2012]. The functions $\eta_{1}$ and $\eta_{2}$ used there become now

and

$$
\eta_{1}(\gamma)=\mathscr{F}_{C_{*}+\gamma}\left(u_{c_{*}+\gamma}\right)-\mathscr{F}_{c_{*}}\left(U_{c_{*}}\right)-\gamma{\left.\frac{d \mathscr{F}_{c}}{d c} U_{c}\right)}_{\mid c=c_{*}} \sim-\frac{\gamma^{3}}{6} \ddot{P}_{*}
$$

$$
\eta_{2}(\gamma)=\frac{d \eta_{1}}{d \gamma}=-P\left(U_{c_{*}+\gamma}\right)+P\left(U_{c_{*}}\right) \sim-\frac{\gamma^{2}}{2} \ddot{P}_{*} .
$$

In order to clarify the dualities used by Maeda, we provide some elements of the proof adapted to our context.

Lemma 4.1. There exists $\gamma_{0}>0$ small and $\sigma:\left(-\gamma_{0},+\gamma_{0}\right) \rightarrow \mathbb{R}$ with $\sigma(\gamma) \sim-\gamma^{2} \ddot{P}_{*} /\left(2\left\|U_{*}\right\|_{H}^{2}\right)$ and such that, for any $\gamma \in\left(-\gamma_{0},+\gamma_{0}\right)$,

$$
P_{\mathrm{hy}}\left(u_{c_{*}+\gamma}+\sigma(\gamma) \mathbb{B} u_{c_{*}+\gamma}\right)=P_{\mathrm{hy}}\left(u_{*}\right) .
$$

Proof. We have

$$
\begin{aligned}
P_{\text {hy }}\left(u_{c_{*}+\gamma}+\sigma \mathbb{B} u_{c_{*}+\gamma}\right) & =\frac{1}{2}\left(\mathbb{B} u_{c_{*}+\gamma}+\sigma u_{c_{*}+\gamma}, u_{c_{*}+\gamma}+\sigma \mathbb{B} u_{c_{*}+\gamma}\right)_{H} \\
& =P_{\text {hy }}\left(u_{c_{*}+\gamma}\right)+\sigma\left\|u_{c_{*}+\gamma}\right\|_{H}^{2}+\sigma^{2} P_{\text {hy }}\left(u_{c_{*}+\gamma}\right) .
\end{aligned}
$$


Since $\left\|u_{*}\right\|_{H}^{2} \neq 0$, the conclusion follows from an easy implicit function argument near $\sigma=\gamma=0$. In [Maeda 2012], the linear mapping $B$ is seen from $X$ to $X^{*}$, but, here, there is no confusion in defining $u_{c_{*}+\gamma}+\sigma \mathbb{B} u_{c_{*}+\gamma} \in H=L^{2} \times L^{2}$.

We define, for $\gamma \in\left(-\gamma_{0},+\gamma_{0}\right)$,

$$
\mathcal{W}(\gamma) \equiv u_{c_{*}+\gamma}+\sigma(\gamma) \mathbb{B} u_{c_{*}+\gamma}
$$

which then satisfies $P_{\text {hy }}(\mathcal{W}(\gamma))=P_{\text {hy }}\left(\cup_{*}\right)$ by construction.

Lemma 4.2. As $\gamma \rightarrow 0$, we have $\mathscr{F}_{c_{*}}(\mathcal{W}(\gamma))-\mathscr{F}_{c_{*}}\left(u_{c_{*}}\right) \sim-\left(\gamma^{3} / 6\right) \ddot{P}_{*}$.

Proof. Using that $\mathscr{F}_{c_{*}+\gamma}^{\prime}\left(u_{c_{*}+\gamma}\right)=0, P_{\mathrm{hy}}(\mathcal{W}(\gamma))=P_{\mathrm{hy}}\left(U_{c_{*}}\right)=-\left(d \mathscr{F}_{c}\left(U_{c}\right) / d c\right)_{\mid c=c_{*}}$ and $\sigma(\gamma)=$ $\mathrm{O}\left(\gamma^{2}\right)$, we have by the Taylor expansion

$$
\begin{aligned}
\mathscr{F}_{c_{*}}(\mathcal{W}(\gamma))-\mathscr{F}_{c_{*}}\left(U_{c_{*}}\right) & =\mathscr{F}_{c_{*}+\gamma}\left(u_{c_{*}+\gamma}+\sigma(\gamma) \mathbb{B} u_{c_{*}+\gamma}\right)-\mathscr{F}_{c_{*}}\left(U_{c_{*}}\right)+\gamma P_{\mathrm{hy}}(\mathcal{W}(\gamma)) \\
& =\mathscr{F}_{c_{*}+\gamma}\left(u_{c_{*}+\gamma}\right)-\mathscr{F}_{c_{*}}\left(u_{c_{*}}\right)-\gamma \frac{d \mathscr{F}_{c}\left(U_{c}\right)}{d c} \mid c=c_{*}+\sigma\left(\gamma^{4}\right) \sim-\frac{\gamma^{3}}{6} \ddot{P}_{*},
\end{aligned}
$$

as wished.

We recall that we have defined the tubular neighborhood $\sigma_{\varepsilon}=\left\{\mathscr{V} \in X\right.$, inf $\left.y \in \mathbb{R}\left\|\mathscr{V}-\mathcal{U}_{*}(\cdot-y)\right\|_{X}<\varepsilon\right\}$.

Lemma 4.3. For $\varepsilon>0$ small enough, there exist four $\mathscr{b}^{1}$ mappings $\bar{\gamma}, \alpha, \bar{y}: \mathrm{O}_{\varepsilon} \rightarrow \mathbb{R}$ and $\vartheta: \mathscr{O}_{\varepsilon} \rightarrow X$, satisfying, for $u \in \mathbb{O}_{\varepsilon}$,

$$
u(\cdot-\bar{y}(u))=\mathscr{W}(\bar{\gamma}(u))+\vartheta(u)+\alpha(u) \mathbb{B} u_{c_{*}+\bar{\gamma}(u)}
$$

and the orthogonality relations

$$
\left(\vartheta(\vartheta), \partial_{x} u_{c_{*}+\bar{\gamma}(u)}\right)_{H}=\left(\vartheta(u),\left[\partial_{c} u_{c}\right]_{\mid c=c_{*}+\bar{\gamma}(u)}\right)_{H}=\left(\vartheta(\vartheta), \mathbb{B} u_{c_{*}+\bar{\gamma}(u)}\right)_{H}=0 .
$$

Finally, $\square^{-1} \bar{\gamma}^{\prime} \in H^{2} \times H^{1}$ and $\square_{H^{1}}^{-1} \partial \bar{\gamma} / \partial \eta \in H^{4}$.

Proof. We consider the mapping $G: X \times \mathbb{R} \times\left(-\gamma_{0},+\gamma_{0}\right) \times \mathbb{R} \rightarrow \mathbb{R}^{3}$ defined by

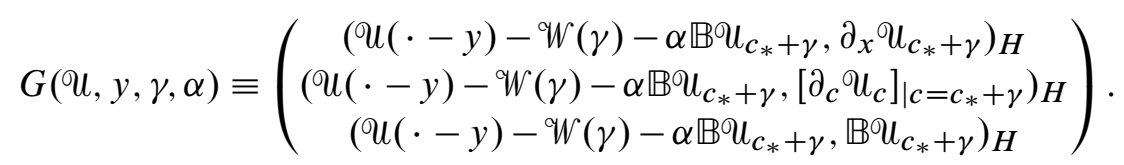

Then $G\left(U_{*}, 0,0,0\right)=0$ since $\mathscr{W}(0)=U_{*}$. In order to show that $G$ is of class $\mathscr{C}^{1}$, we have to pay attention to the translation term $u(\cdot-y)$, since differentiation in $y$ requires $u \in H^{1} \times H^{1}$ whereas we only have $U \in X=H^{1} \times L^{2}$. It thus suffices to write

$$
G(\mathcal{u}, y, \gamma, \alpha)=\left(\begin{array}{c}
\left(\mathcal{u}, \partial_{x} u_{c_{*}+\gamma}(\cdot+y)\right)_{H}-\left(\mathcal{W}(\gamma)+\alpha \mathbb{B} u_{c_{*}+\gamma}, \partial_{x} u_{c_{*}+\gamma}\right)_{H} \\
\left(\mathcal{u},\left[\partial_{c} u_{c}\right]_{\mid c=c_{*}+\gamma}(\cdot+y)\right)_{H}-\left(\mathcal{W}(\gamma)+\alpha \mathbb{B} u_{c_{*}+\gamma},\left[\partial_{c} u_{c}\right]_{\mid c=c_{*}+\gamma}\right)_{H} \\
\left(\mathcal{u}, \mathbb{B} u_{c_{*}+\gamma}(\cdot+y)\right)_{H}-\left(\mathcal{W}(\gamma)+\alpha \mathbb{B} u_{c_{*}+\gamma}, \mathbb{B} u_{c_{*}+\gamma}\right)_{H}
\end{array}\right)
$$


to see that $G$ is indeed of class $\mathscr{C}^{1}$ on $X \times \mathbb{R} \times\left(-\gamma_{0},+\gamma_{0}\right) \times \mathbb{R}$ since $c \mapsto \boldsymbol{U}_{c}$ is smooth. Moreover, using that $\partial_{\gamma} W_{\mid \gamma=0}=\left[\partial_{c} u_{c}\right]_{\mid c=c_{*}}$, we infer

$$
\frac{\partial G}{\partial(y, \gamma, \alpha)}\left(u_{*}, 0,0,0\right)=\left(\begin{array}{ccc}
\left(u_{*}, \partial_{x}^{2} u_{*}\right)_{H} & -\left(\left[\partial_{c} u_{c}\right]_{\mid c=c_{*}}, \partial_{x} u_{*}\right)_{H} & -\left(\mathbb{B} u_{*}, \partial_{x} u_{*}\right)_{H} \\
-\left(u_{*}, \partial_{x}\left[\partial_{c} u_{c}\right]_{\mid c=c_{*}}\right)_{H} & -\left\|\left[\partial_{c} u_{c}\right]_{\mid c=c_{*}}\right\|_{H}^{2} & -\left(\mathbb{B} u_{*},\left[\partial_{c} u_{c}\right]_{\mid c=c_{*}}\right)_{H} \\
-\left(u_{*}, \mathbb{B} \partial_{x} u_{*}\right)_{H} & -\left(\left[\partial_{c} u_{c}\right]_{\mid c=c_{*}}, \mathbb{B} u_{*}\right)_{H} & -\left\|\mathbb{B} u_{*}\right\|_{H}^{2}
\end{array}\right) .
$$

At this stage, the argument in [Maeda 2012] is to use that

$$
\left(\left[\partial_{c} u_{c}\right]_{\mid c=c_{*}}, \partial_{x} u_{*}\right)_{H}=-\left(\partial_{x}\left[\partial_{c} u_{c}\right]_{\mid c=c_{*}}, u_{*}\right)_{H}=0,
$$

which is assumption 2(iii) there. This equality holds true for us since we have chosen $\boldsymbol{U}_{c}$ even for any $c$ (close to $\left.c_{*}\right)$. Furthermore, $\left(u_{*}, \partial_{x}^{2} u_{*}\right)_{H}=-\left\|\partial_{x} u_{*}\right\|_{H}^{2}$ by integration by parts, $\left(\mathbb{B} u_{*}, \partial_{x} u_{*}\right)_{H}=$ $\left(u_{*}, \mathbb{B} \partial_{x} u_{*}\right)_{H}=0$ since $\mathbb{B} \partial_{x}=J$ is skew-adjoint, and $\left(\mathbb{B} u_{*},\left[\partial_{c} u_{c}\right]_{\mid c=c_{*}}\right)_{H}=\partial_{c}\left[P_{\text {hy }}\left(u_{c}\right)\right]_{\mid c=c_{*}}=0$ by hypothesis. Therefore,

$$
\frac{\partial G}{\partial(y, \gamma, \alpha)}\left(u_{*}, 0,0,0\right)=\left(\begin{array}{ccc}
-\left\|\partial_{x} u_{c_{*}}\right\|_{H}^{2} & 0 & 0 \\
0 & -\left\|\left[\partial_{c} u_{c}\right]_{\mid c=c_{*}}\right\|_{H}^{2} & 0 \\
0 & 0 & -\left\|\mathbb{B} u_{*}\right\|_{H}^{2}
\end{array}\right)
$$

is invertible; thus the implicit function theorem provides three real-valued functions $y, \gamma$ and $\underline{\alpha}$, defined near $u_{*}$ (in $\left.X\right)$ and with $y\left(u_{*}\right)=\gamma\left(u_{*}\right)=\underline{\alpha}\left(u_{*}\right)=0$, such that $G(u, y(u), \gamma(\tilde{u}), \underline{\alpha}(u))=0$. These functions are extended to $\mathcal{O}_{\varepsilon}$ (for $\varepsilon$ small enough) by the formulas $\bar{y}(u) \equiv \underline{y}(u(\cdot-y))+y$, $\bar{\gamma}(u) \equiv \underline{\gamma}(u(\cdot-y))$ and $\alpha(u) \equiv \underline{\alpha}(u(\cdot-y))$ for any $y \in \mathbb{R}$ such that $u(\cdot-y)$ lies in the neighborhood of $u_{*}$ where $\underline{y}, \underline{\gamma}$ and $\underline{\alpha}$ are defined. Consequently, the mapping

$$
\vartheta(u) \equiv \mho(\cdot-\bar{y}(u))-\mathscr{W}(\bar{\gamma}(u))-\bar{\alpha}(u) \mathbb{B} u_{c_{*}+\bar{\gamma}(u)}
$$

is orthogonal in $H$ to $\partial_{x} u_{c_{*}+\bar{\gamma}(u)},\left[\partial_{c} u_{c}\right]_{\mid c=c_{*}+\bar{\gamma}(u)}$ and $\mathbb{B} u_{c_{*}+\bar{\gamma}(u)}$, as desired. Since $f$ is assumed of class $\mathscr{C}^{2}$, we have $u_{c} \in H^{4}$ and the regularities $\rrbracket^{-1} \bar{\gamma}^{\prime} \in H^{2} \times H^{1}$ and ${ }_{H^{1}}^{-1} \partial \bar{\gamma} / \partial \eta \in H^{4}$ follow easily.

Remark 4.4. We would like to point out that, in [ibid., Lemma 3], it is claimed that " $w(u)$ " is orthogonal to " $\partial_{\omega} \phi_{\omega+\Lambda(u)}$ " (we refer to the notations there). However, since " $T(\theta(u))-\Psi(\Lambda(u))$ " is already orthogonal to " $\partial_{\omega} \phi_{\omega+\Lambda(u)}$ " by construction, this is equivalent to " $\left\langle B \phi_{\omega+\Lambda(u)}, \partial_{\omega} \phi_{\omega+\Lambda(u)}\right\rangle=0$ ", or " $\partial_{\omega^{\prime}}\left[Q\left(\phi_{\omega^{\prime}}\right)\right]=0$ " at " $\omega^{\prime}=\omega+\Lambda(u)$ ". We have not understood why this should happen since, in general, for the function $\omega^{\prime} \mapsto Q\left(\phi_{\omega^{\prime}}\right)$, the point $\omega$ is the only local critical point. For this reason, we have added a component to the original mapping $G$ in [ibid.]. Let us observe that, then, Lemma 3 in [ibid.] uses the assumption " $d^{\prime \prime}(\omega)=0$ ". On the other hand, the derivative of $G$ in [ibid.] assumes " $u \in D\left(T^{\prime}(0)\right)$ ", for otherwise the expression " $G_{1,1}(u, \theta, \Lambda)=\left\langle T^{\prime}(0) T(\theta) u, T^{\prime}(0) \phi_{\omega+\Lambda}\right\rangle$ ", for instance, is meaningless. We have therefore given some details showing clearly the smoothness of $G$.

We now prove a lemma which shows that the quadratic functional associated with $\mathscr{F}_{*}^{\prime \prime}$ gives a good control on $\vartheta(u)$ thanks to the orthogonality conditions on this function. This result is in the spirit of Lemma 7 in [Ohta 2011]. 
Lemma 4.5. There exist $0<\gamma_{1} \leq \gamma_{0}$ and $K_{0}>0$ such that, if $\gamma \in\left(-\gamma_{1},+\gamma_{1}\right)$ and if $\vartheta \in X$ satisfies

$$
\left(\vartheta, \partial_{x} u_{c_{*}+\gamma}\right)_{H}=\left(\vartheta,\left[\partial_{c} u_{c}\right]_{\mid c=c_{*}+\gamma}\right)_{H}=\left(\vartheta, \mathbb{B} u_{c_{*}+\gamma}\right)_{H}=0,
$$

then $\left\langle\mathscr{F}_{c_{*}+\gamma}^{\prime \prime}\left(\vartheta_{c_{*}+\gamma}\right) \vartheta, \vartheta\right\rangle_{X^{*}, X} \geq K_{0}\|\vartheta\|_{X}^{2}$.

Proof. As a first step, we prove that, if $\vartheta \in X$ satisfies $\vartheta \neq 0$,

$$
\left(\vartheta, \partial_{x} u_{*}\right)_{H}=\left(\vartheta,\left[\partial_{c} u_{c}\right]_{\mid c=c_{*}}\right)_{H}=\left(\vartheta, \mathbb{B} u_{*}\right)_{H}=0,
$$

then $\left\langle\mathscr{F}_{*}^{\prime \prime}\left(U_{*}\right) \vartheta, \vartheta\right\rangle_{X^{*}, X}>0$. Indeed, assume that $\left\langle\mathscr{F}_{*}^{\prime \prime}\left(U_{*}\right) \vartheta, \vartheta\right\rangle_{X^{*}, X} \leq 0$. Let $\chi \in X$ be a negative eigenvector of $\mathscr{F}_{*}^{\prime \prime}$. We claim that we can not have $\left((\vartheta, \chi)_{H},\left(\left[\partial_{c} \varkappa_{c}\right]_{\mid c=c_{*}}, \chi\right)_{H}\right)=(0,0)$. Otherwise, $(\vartheta, \chi)_{H}=0$ implies that $\vartheta$ is $L^{2}$-orthogonal to $\chi$, which is the eigenvector associated with the only negative eigenvalue $-\mu_{0}$ of $\mathscr{F}_{*}^{\prime \prime}$ seen as an unbounded operator on $L^{2}$; thus $\left\langle\mathscr{F}_{*}^{\prime \prime}\left(U_{*}\right) \vartheta, \vartheta\right\rangle_{X^{*}, X} \geq 0$, and, since we assume equality, this means that $\vartheta$ belongs to the kernel of $\mathscr{F}_{*}^{\prime \prime}\left(U_{*}\right)$, which is spanned by $U_{*}=\partial_{x} U_{*}$, but the condition $\left(\vartheta, \partial_{x} U_{*}\right)_{H}=0$ then implies $\vartheta=0$, a contradiction. Therefore, there exists $(a, b) \in \mathbb{R}^{2}$ such that $(a, b) \neq(0,0)$ and $\left(a\left[\partial_{c} \mho_{c}\right]_{\mid c=c_{*}}+b \vartheta, \chi\right)_{H}=0$. The nonzero vector $p \equiv a\left[\partial_{c} u_{c}\right]_{\mid c=c_{*}}+b \vartheta$ then satisfies $(p, \chi)_{H}=0$ and $\left(p, J \cup_{*}\right)_{H}=a\left(\left[\partial_{c} U_{c}\right]_{\mid c=c_{*}}, J \cup_{*}\right)_{H}+b\left(\vartheta, J \cup_{*}\right)_{H}=0$, so that $\left\langle\mathscr{F}_{*}^{\prime \prime}\left(U_{*}\right) p, p\right\rangle_{X^{*}, X}>0$. Here, we have used once again that $\left(\left[\partial_{c} u_{c}\right]_{\mid c=c_{*}}, J U_{*}\right)_{H}=0$ since the left vector is an even function and the right vector an odd function. However, in view of the equality $\left\langle\mathscr{F}_{*}^{\prime \prime}\left(\cup_{*}\right)\left[\partial_{c} U_{c}\right]_{\mid c=c_{*}}, \phi\right\rangle_{X^{*}, X}=\left(\mathbb{B} U_{*}, \phi\right)_{H}$, valid for any $\phi \in X$ (which follows from differentiation of $E_{\text {hy }}^{\prime}\left(U_{c}\right)=c P_{\text {hy }}^{\prime}\left(\vartheta_{c}\right)=c\left(\mathbb{B} u_{c}, \cdot\right)_{H}$ at $\left.c=c_{*}\right)$, we have

$$
\left\langle\mathscr{F}_{*}^{\prime \prime}\left(\mathcal{U}_{*}\right)\left[\partial_{c} u_{c}\right]_{\mid c=c_{*}}, \vartheta\right\rangle_{X^{*}, X}=\left(\mathbb{B}\left[\partial_{c} u_{c}\right]_{\mid c=c_{*}}, \vartheta\right)_{H}=0 .
$$

As a consequence,

$$
\begin{aligned}
& 0<\left\langle\mathscr{F}_{*}^{\prime \prime}\left(\cup_{*}\right) p, p\right\rangle_{X^{*}, X}=a^{2}\left\langle\mathscr{F}_{*}^{\prime \prime}\left(u_{*}\right)\left[\partial_{c} u_{c}\right]_{\mid c=c_{*}},\left[\partial_{c} u_{c}\right]_{\mid c=c_{*}}\right\rangle_{X^{*}, X}+b^{2}\left\langle\mathscr{F}_{*}^{\prime \prime}\left(\vartheta_{*}\right) \vartheta, \vartheta\right\rangle_{X^{*}, X} \\
& =a^{2}\left(\mathbb{B} u_{*},\left[\partial_{c} u_{c}\right]_{\mid c=c_{*}}\right)_{H}+b^{2}\left\langle\mathscr{F}_{*}^{\prime \prime}\left(\cup_{*}\right) \vartheta, \vartheta\right\rangle_{X^{*}, X}=b^{2}\left\langle\mathscr{F}_{*}^{\prime \prime}\left(\vartheta_{*}\right) \vartheta, \vartheta\right\rangle_{X^{*}, X},
\end{aligned}
$$

since $\left(\mathbb{B} U_{*},\left[\partial_{c} U_{c}\right]_{\mid c=c_{*}}\right)_{H}=\partial_{c}\left[P_{\mathrm{hy}}\left(U_{c}\right)\right]_{\mid c=c_{*}}=0$ in our situation. We reach a contradiction since the right-hand side is supposed $\leq 0$.

We now prove the lemma by contradiction, and then assume that there exist sequences $\left(\vartheta_{n}\right)_{n \geq 1} \in X$ and $\left(\gamma_{n}\right)_{n \geq 1} \in\left(0, \gamma_{0}\right)$ such that $\gamma_{n} \rightarrow 0,\left\|\vartheta_{n}\right\|_{X}^{2}=1$ and

$$
\left(\vartheta_{n}, \partial_{x} u_{c_{*}+\gamma_{n}}\right)_{H}=\left(\vartheta_{n},\left[\partial_{c} u_{c}\right]_{\mid c=c_{*}+\gamma_{n}}\right)_{H}=\left(\vartheta_{n}, \mathbb{B} u_{c_{*}+\gamma_{n}}\right)_{H}=0,
$$

but $\left\langle\mathscr{F}_{\mathcal{F}_{*}+\gamma_{n}}^{\prime \prime}\left(\boldsymbol{U}_{\mathcal{C}_{*}+\gamma_{n}}\right) \vartheta_{n}, \vartheta_{n}\right\rangle_{X^{*}, X} \rightarrow 0$. Possibly passing to a subsequence, we may assume the existence of some $\vartheta=(\zeta, v) \in X$ such that $\vartheta_{n} \equiv\left(\zeta_{n}, v_{n}\right) \rightarrow \vartheta$ in $X=H^{1} \times L^{2}$. We then show the lower semicontinuity of $\left\langle\mathscr{F}_{*}^{\prime \prime}\left(U_{*}\right) \vartheta, \vartheta\right\rangle_{X^{*}, X}$. This is roughly a verification of part of assumption (A3) in [Ohta 2011], used in Lemma 7 there. By the compact Sobolev embedding, we may assume $\zeta_{n} \rightarrow \zeta$ in $L_{\text {loc }}^{\infty}(\mathbb{R})$. 
A straightforward computation gives

$$
\begin{aligned}
\left\langle\mathscr{F}_{c_{*}+\gamma}^{\prime \prime}\left(u_{c_{*}+\gamma}\right) \vartheta, \vartheta\right\rangle_{X^{*}, X}= & \int_{\mathbb{R}} \frac{\left(\partial_{x} \zeta\right)^{2}}{2\left(r_{0}^{2}+\eta_{c_{*}+\gamma}\right)}-\frac{\partial_{x} \zeta \partial_{x} \eta_{c_{*}+\gamma}}{\left(r_{0}^{2}+\eta_{c_{*}+\gamma}\right)^{2}}+\frac{\zeta^{2}\left(\partial_{x} \eta_{c_{*}+\gamma}\right)^{2}}{4\left(r_{0}^{2}+\eta_{c_{*}+\gamma}\right)^{3}} \\
& +2\left(r_{0}^{2}+\eta_{c_{*}+\gamma}\right) v^{2}+2\left(2 u_{c_{*}+\gamma}-\left(c_{*}+\gamma\right)\right) v \zeta-f^{\prime}\left(r_{0}^{2}+\eta_{c_{*}+\gamma}\right) \zeta^{2} d x
\end{aligned}
$$

Since $r_{0}^{2}+\eta_{c_{*}+\gamma_{n}}$ remains bounded away from zero uniformly and $\eta_{c_{*}+\gamma_{n}} \rightarrow \eta_{c_{*}}$ in $W^{1, \infty}(\mathbb{R}) \cap H^{1}(\mathbb{R})$ as $n \rightarrow+\infty$, the weak convergence $\zeta_{n} \rightarrow \zeta$ in $H^{1}$ implies

$$
\begin{aligned}
& \int_{\mathbb{R}} \frac{\left(\partial_{x} \zeta\right)^{2}}{2\left(r_{0}^{2}+\eta_{c_{*}}\right)}-\frac{\partial_{x} \zeta \partial_{x} \eta_{c_{*}}}{\left(r_{0}^{2}+\eta_{c_{*}}\right)^{2}}+\frac{\zeta^{2}\left(\partial_{x} \eta_{c_{*}}\right)^{2}}{4\left(r_{0}^{2}+\eta_{c_{*}}\right)^{3}} d x \\
& \leq \lim _{n \rightarrow+\infty} \int_{\mathbb{R}} \frac{\left(\partial_{x} \zeta_{n}\right)^{2}}{2\left(r_{0}^{2}+\eta_{c_{*}+\gamma_{n}}\right)}-\frac{\partial_{x} \zeta_{n} \partial_{x} \eta_{c_{*}+\gamma_{n}}}{\left(r_{0}^{2}+\eta_{c_{*}+\gamma_{n}}\right)^{2}}+\frac{\zeta_{n}^{2}\left(\partial_{x} \eta_{c_{*}+\gamma_{n}}\right)^{2}}{4\left(r_{0}^{2}+\eta_{c_{*}+\gamma_{n}}\right)^{3}} d x .
\end{aligned}
$$

For the remaining terms, we write, for some $R>0$ to be determined later,

$$
\begin{aligned}
\int_{\mathbb{R}} 2\left(r_{0}^{2}+\eta_{c_{*}+\gamma_{n}}\right) v_{n}^{2}+2\left(2 u_{c_{*}+\gamma_{n}}-\left(c_{*}+\gamma_{n}\right)\right) v_{n} \zeta_{n}-f^{\prime}\left(r_{0}^{2}+\eta_{c_{*}+\gamma_{n}}\right) \zeta_{n}^{2} d x \\
=\int_{\mathbb{R}} 2\left[\left(r_{0}^{2}+\eta_{c_{*}+\gamma_{n}}\right)^{1 / 2} v_{n}+\frac{\left(2 u_{c_{*}+\gamma_{n}}-\left(c_{*}+\gamma_{n}\right)\right) \zeta_{n}}{2\left(r_{0}^{2}+\eta_{c_{*}+\gamma_{n}}\right)^{1 / 2}}\right]^{2} d x \\
\quad+\int_{|x| \leq R}+\int_{|x| \geq R} \frac{1}{2}\left(-\frac{\left(2 u_{c_{*}+\gamma_{n}}-\left(c_{*}+\gamma_{n}\right)\right)^{2}}{r_{0}^{2}+\eta_{c_{*}+\gamma_{n}}}-2 f^{\prime}\left(r_{0}^{2}+\eta_{c_{*}+\gamma_{n}}\right)\right) \zeta_{n}^{2} d x
\end{aligned}
$$

For the first integral, we may use that $\left(\zeta_{n}, v_{n}\right) \rightarrow(\zeta, v)$ in $L^{2} \times L^{2}$ and the fact that $\left(\eta_{c_{*}+\gamma_{n}}, u_{c_{*}+\gamma_{n}}\right)$ converges to $\left(\eta_{*}, u_{*}\right)$ uniformly to deduce

$$
\left(r_{0}^{2}+\eta_{c_{*}+\gamma_{n}}\right)^{1 / 2} v_{n}+\frac{\left(2 u_{c_{*}+\gamma_{n}}-\left(c_{*}+\gamma_{n}\right)\right) \zeta_{n}}{2\left(r_{0}^{2}+\eta_{c_{*}+\gamma_{n}}\right)^{1 / 2}} \rightarrow\left(r_{0}^{2}+\eta_{*}\right)^{1 / 2} v+\frac{\left(2 u_{*}-c_{*}\right) \zeta}{2\left(r_{0}^{2}+\eta_{*}\right)^{1 / 2}} \quad \text { in } L^{2} ;
$$

hence,

$$
\begin{aligned}
& \int_{\mathbb{R}} 2\left[\left(r_{0}^{2}+\eta_{*}\right)^{1 / 2} v+\frac{\left(2 u_{*}-c_{*}\right) \zeta}{2\left(r_{0}^{2}+\eta_{*}\right)^{1 / 2}}\right]^{2} d x \\
& \leq \underset{n \rightarrow+\infty}{\underline{\lim }} \int_{\mathbb{R}} 2\left[\left(r_{0}^{2}+\eta_{c_{*}+\gamma_{n}}\right)^{1 / 2} v_{n}+\frac{\left(2 u_{c_{*}+\gamma_{n}}-\left(c_{*}+\gamma_{n}\right)\right) \zeta_{n}}{2\left(r_{0}^{2}+\eta_{c_{*}+\gamma_{n}}\right)^{1 / 2}} d x .\right.
\end{aligned}
$$

Since $\zeta_{n} \rightarrow \zeta$ in $L^{\infty}([-R,+R])$ and $\left(u_{c_{*}+\gamma_{n}}, \eta_{c_{*}+\gamma_{n}}\right) \rightarrow\left(u_{*}, \eta_{*}\right)$ uniformly, it follows that

$$
\begin{aligned}
\int_{|x| \leq R} \frac{1}{2}\left(-\frac{\left(2 u_{*}-c_{*}\right)^{2}}{r_{0}^{2}+\eta_{*}}-\right. & \left.2 f^{\prime}\left(r_{0}^{2}+\eta_{*}\right)\right) \zeta^{2} d x \\
& =\lim _{n \rightarrow+\infty} \int_{|x| \leq R} \frac{1}{2}\left(-\frac{\left(2 u_{c_{*}+\gamma_{n}}-\left(c_{*}+\gamma_{n}\right)\right)^{2}}{r_{0}^{2}+\eta_{c_{*}+\gamma_{n}}}-2 f^{\prime}\left(r_{0}^{2}+\eta_{c_{*}+\gamma_{n}}\right)\right) \zeta_{n}^{2} d x
\end{aligned}
$$

For the last integral, we have to use the decay at infinity of $\eta_{c_{*}+\gamma}$ and $u_{c_{*}+\gamma}$ uniformly for $|\gamma|$ small. 
This gives

$$
-\frac{\left(2 u_{c_{*}+\gamma_{n}}-\left(c_{*}+\gamma_{n}\right)\right)^{2}}{r_{0}^{2}+\eta_{c_{*}+\gamma_{n}}}-2 f^{\prime}\left(r_{0}^{2}+\eta_{c_{*}+\gamma_{n}}\right) \rightarrow \frac{\mathfrak{c}_{s}^{2}-c_{*}^{2}}{r_{0}^{2}}
$$

as $|x| \rightarrow+\infty$, uniformly in $n$. Since $0<c_{*}<\mathfrak{c}_{s}$, there exist some small $\delta>0$ and some $R>0$ large such that, for any $n$ and any $x$ with $|x| \geq R$,

$$
-\frac{\left(2 u_{c_{*}+\gamma_{n}}-\left(c_{*}+\gamma_{n}\right)\right)^{2}}{r_{0}^{2}+\eta_{c_{*}}+\gamma_{n}}-2 f^{\prime}\left(r_{0}^{2}+\eta_{c_{*}+\gamma_{n}}\right) \geq \delta .
$$

In particular, since $\zeta_{n} \rightarrow \zeta$ in $L^{2}$,

$$
\mathbf{1}_{|x| \geq R}\left(-\frac{\left(2 u_{c_{*}+\gamma_{n}}-\left(c_{*}+\gamma_{n}\right)\right)^{2}}{r_{0}^{2}+\eta_{c_{*}+\gamma_{n}}}-2 f^{\prime}\left(r_{0}^{2}+\eta_{c_{*}+\gamma_{n}}\right)\right)^{1 / 2} \zeta_{n} \rightarrow \mathbf{1}_{|x| \geq R}\left(-\frac{\left(2 u_{*}-c_{*}\right)^{2}}{r_{0}^{2}+\eta_{*}}-2 f^{\prime}\left(r_{0}^{2}+\eta_{*}\right)\right)^{1 / 2} \zeta
$$

in $L^{2}$; thus

$$
\begin{aligned}
\int_{|x| \geq R} \frac{1}{2}\left(-\frac{\left(2 u_{*}-c_{*}\right)^{2}}{r_{0}^{2}+\eta_{*}}-2 f^{\prime}\left(r_{0}^{2}+\eta_{*}\right)\right) \zeta^{2} d x \\
\leq \varliminf_{n \rightarrow+\infty} \int_{|x| \geq R} \frac{1}{2}\left(-\frac{\left(2 u_{c_{*}+\gamma_{n}}-\left(c_{*}+\gamma_{n}\right)\right)^{2}}{r_{0}^{2}+\eta_{c_{*}+\gamma_{n}}}-2 f^{\prime}\left(r_{0}^{2}+\eta_{c_{*}+\gamma_{n}}\right)\right) \zeta_{n}^{2} d x
\end{aligned}
$$

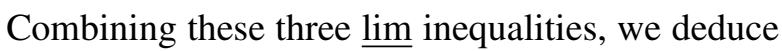

$$
\left\langle\mathscr{F}_{*}^{\prime \prime}\left(U_{*}\right) \vartheta, \vartheta\right\rangle_{X^{*}, X} \leq \lim _{n \rightarrow+\infty}\left\langle\mathscr{F}_{c_{*}+\gamma_{n}}^{\prime \prime}\left(\vartheta_{c_{*}+\gamma_{n}}\right) \vartheta_{n}, \vartheta_{n}\right\rangle_{X^{*}, X}=0
$$

Turning back to our sequence $\left(\vartheta_{n}, \gamma_{n}\right)$, we may pass to the limit in (18):

$$
\left(\vartheta, \partial_{x} u_{*}\right)_{H}=\left(\vartheta,\left[\partial_{c} u_{c}\right]_{\mid c=c_{*}}\right)_{H}=\left(\vartheta, \mathbb{B} U_{*}\right)_{H}=0 .
$$

Comparing with (23), we deduce from our first claim that $\vartheta=0$. This means that we must have equality in all the above lim inequalities. In particular, the weak convergence (22) is actually strong; thus $\zeta_{n} \rightarrow \zeta=0$ in $L^{2}(\mathbb{R})$ (the strong convergence in $\{|x| \leq R\}$ being already known since $\zeta_{n} \rightarrow \zeta$ in $L_{\text {loc }}^{\infty}(\mathbb{R})$ ). Going back to the equality in (19) thus provides $\partial_{x} \zeta_{n} \rightarrow \partial_{x} \zeta=0$ in $L^{2}(\mathbb{R})$, since $r_{0}^{2}+\eta_{c_{*}+\gamma_{n}}$ remains uniformly bounded away from zero, and by weak convergence,

$$
0=\int_{\mathbb{R}} \frac{\zeta^{2}\left(\partial_{x} \eta_{c_{*}}\right)^{2}}{4\left(r_{0}^{2}+\eta_{c_{*}}\right)^{3}} d x=\lim _{n \rightarrow+\infty} \int_{\mathbb{R}} \frac{\partial_{x} \zeta_{n} \partial_{x} \eta_{c_{*}+\gamma_{n}}}{\left(r_{0}^{2}+\eta_{c_{*}+\gamma_{n}}\right)^{2}} d x
$$

Finally, the equality in (21) means that (20) is actually a strong convergence; that is, $v_{n} \rightarrow v=0$ in $L^{2}$ since $\zeta_{n} \rightarrow \zeta$ in $L^{2}$. The contradiction then follows: $1=\left\|\vartheta_{n}\right\|_{X}^{2}=\left\|\zeta_{n}\right\|_{L^{2}}^{2}+\left\|\partial_{x} \zeta_{n}\right\|_{L^{2}}^{2}+\left\|v_{n}\right\|_{L^{2}}^{2} \rightarrow 0$.

Remark 4.6. This result is also Lemma 7 in [Maeda 2012], and is said to be Lemma 7 in [Ohta 2011]. However, the hypotheses of Lemma 7 in [Ohta 2011] are not satisfied, and in particular assumption (B3) there. It is natural to believe that this assumption is satisfied is most physical situations, but it is not clear whether it always holds true in the general framework of [Maeda 2012] without further hypothesis.

The next lemma provides a control for $\alpha(U)$. 
Lemma 4.7. Assume $\varepsilon>0$ small enough. Then, there exists $K>0$ such that, for any $\mathcal{U} \in \mathrm{O}_{\varepsilon}$ satisfying $P_{\mathrm{hy}}(u)=P_{\mathrm{hy}}\left(u_{*}\right)$, we have

$$
|\alpha(\vartheta)| \leq K\left(\bar{\gamma}^{2}(u)\|\vartheta(u)\|_{X}+\|\vartheta(u)\|_{X}^{2}\right) .
$$

Proof. It is the same as in [Maeda 2012, Lemma 8], but we give it for completeness. We expand and use that $\mathbb{B}^{2}=\operatorname{Id}_{2}$ and the definition $\mathcal{W}(\gamma) \equiv u_{c_{*}+\gamma}+\sigma(\gamma) \mathbb{B} u_{c_{*}+\gamma}$ for the second line:

$$
\begin{aligned}
& P_{\text {hy }}\left(u_{*}\right)=P_{\text {hy }}(u)=P_{\text {hy }}(u(\cdot-\bar{y}(u)))=P_{\text {hy }}\left(\mathcal{W}(\bar{\gamma}(u))+\vartheta(u)+\alpha(\vartheta) \mathbb{B} u_{c_{*}+\bar{\gamma}(u)}\right) \\
& =P_{\mathrm{hy}}(\mathcal{W}(\bar{\gamma}(\mathcal{u})))+P_{\mathrm{hy}}(\vartheta(\vartheta))+\alpha^{2}(\vartheta) P_{\mathrm{hy}}\left(\mathbb{B} u_{c_{*}+\bar{\gamma}(u)}\right)+\alpha(u)\left(\mathbb{B} \vartheta(\vartheta), \mathbb{B} u_{c_{*}+\bar{\gamma}(u)}\right)_{H} \\
& +\left(\mathbb{B} u_{c_{*}+\bar{\gamma}(U)}, \vartheta(u)\right)_{H}+\alpha(\mho)\left(\mathbb{B} u_{c_{*}+\bar{\gamma}(u)}, \mathbb{B} u_{c_{*}+\bar{\gamma}(u)}\right)_{H} \\
& +\sigma(\bar{\gamma}(u))\left(u_{c_{*}+\bar{\gamma}(u)}, \vartheta(u)\right)_{H}+\sigma(\bar{\gamma}(u)) \alpha(u)\left(u_{c_{*}+\bar{\gamma}(u)}, \mathbb{B} u_{c_{*}+\bar{\gamma}(u)}\right)_{H} .
\end{aligned}
$$

Since $P_{\mathrm{hy}}(W(\bar{\gamma}(\mathcal{U})))=P_{\mathrm{hy}}\left(U_{*}\right)$, we infer

$$
-\alpha(u)\left[\left\|u_{*}\right\|_{H}^{2}+o(1)\right]=\sigma(\bar{\gamma}(u))\left(u_{c_{*}+\bar{\gamma}(u)}, \vartheta(\vartheta)\right)_{H}+P_{\text {hy }}(\vartheta(u))
$$

and the conclusion follows since $\sigma(\gamma)=O\left(\gamma^{2}\right)$ by Lemma 4.1.

Now, we give a lemma useful to estimate $\vartheta(น)$.

Lemma 4.8. Assume $\varepsilon>0$ small enough. Then, there exists $K>0$ such that, for any $\mathcal{U} \in \mathrm{O}_{\varepsilon}$ satisfying $P_{\mathrm{hy}}(u)=P_{\mathrm{hy}}\left(u_{*}\right)$ and $\mathscr{F}_{*}(u)-\mathscr{F}_{*}\left(u_{*}\right)<0$, we have

$$
\|\vartheta(u)\|_{X}^{2} \leq K|\bar{\gamma}(U)|^{3}
$$

In particular, $|\alpha(\mathcal{U})| \leq K|\bar{\gamma}(u)|^{3}$.

Proof. It is the same as in [Maeda 2012, Lemma 9]. Note first that the last assertion is a direct consequence of the first one and Lemma 4.7. Next, we argue by contradiction and assume that there exists a sequence $u_{n} \rightarrow u_{*}$ in $X$ such that $\mathscr{F}_{*}\left(u_{n}\right)-\mathscr{F}_{*}\left(u_{*}\right)<0$ and $\left\|\vartheta\left(u_{n}\right)\right\|_{X}^{2} \gg\left|\bar{\gamma}\left(u_{n}\right)\right|^{3}$. For simplicity, we define $\bar{\gamma}_{n}=\bar{\gamma}\left(u_{n}\right), \vartheta_{n}=\vartheta\left(u_{n}\right), \alpha_{n}=\alpha\left(u_{n}\right)$. Then, by Lemma 4.7, we have $\left|\alpha_{n}\right| \leq K\left(\bar{\gamma}_{n}^{2}\left\|\vartheta_{n}\right\|_{X}+\left\|\vartheta_{n}\right\|_{X}^{2}\right) \leq$ $K\left(\left\|\vartheta_{n}\right\|_{X}^{7 / 3}+\left\|\vartheta_{n}\right\|_{X}^{2}\right)=\mathcal{O}\left(\left\|\vartheta_{n}\right\|_{X}^{2}\right)$. Therefore, by the Taylor expansion and Lemma 4.3, we have

$$
\begin{aligned}
& \mathscr{F}_{*}\left(u_{n}\right)-\mathscr{F}\left(u_{*}\right)=\mathscr{F}_{*}\left(u_{n}\left(\cdot-\bar{y}_{n}\right)\right)-\mathscr{F}\left(u_{*}\right)=\mathscr{F}_{*}\left(\mathscr{W}\left(\bar{\gamma}_{n}\right)+\vartheta_{n}+\alpha_{n} \mathbb{B} u_{c_{*}+\bar{\gamma}_{n}}\right)-\mathscr{F}\left(\mathcal{u}_{*}\right) \\
& =\mathscr{F}_{*}\left(\mathcal{W}\left(\bar{\gamma}_{n}\right)\right)-\mathscr{F}_{*}\left(\mathcal{U}_{*}\right)+\left\langle\mathscr{F}_{*}^{\prime}\left(\mathcal{W}\left(\bar{\gamma}_{n}\right)\right), \vartheta_{n}+\alpha_{n} \mathbb{B} u_{c_{*}+\bar{\gamma}_{n}}\right\rangle_{X^{*}, X} \\
& +\frac{1}{2}\left\langle\mathscr{F}_{*}^{\prime \prime}\left(\mathcal{W}\left(\bar{\gamma}_{n}\right)\right) \vartheta_{n}, \vartheta_{n}\right\rangle_{X^{*}, X}+o\left(\left\|\vartheta\left(u_{n}\right)\right\|_{X}^{2}\right) \text {. }
\end{aligned}
$$

However, by Lemma 4.2, $\mathscr{F}_{c_{*}}(\mathcal{W}(\gamma))-\mathscr{F}_{c_{*}}\left(\mathcal{U}_{c_{*}}\right)=\mathcal{O}\left(|\gamma|^{3}\right)$, and, since $\mathscr{F}_{*}^{\prime}(\mathcal{W}(\gamma))=\mathscr{F}_{*}^{\prime}(\mathscr{W}(0))+o(1)=$ $\mathscr{F}_{*}^{\prime}\left(U_{*}\right)+o(1)=o(1)$, we have $\left\langle\mathscr{F}_{*}^{\prime}\left(\mathcal{W}\left(\bar{\gamma}_{n}\right)\right), \alpha_{n} \mathbb{B} u_{c_{*}+\bar{\gamma}_{n}}\right\rangle_{X^{*}, X}=o\left(\left\|\vartheta_{n}\right\|_{X}^{2}\right)$. Furthermore, using $\mathscr{F}_{*}^{\prime}=\mathscr{F}_{c_{*}+\bar{\gamma}_{n}}^{\prime}+\bar{\gamma}_{n} \mathbb{B}$, the third orthogonality condition in Lemma 4.3 and that $\sigma(\gamma)=\mathcal{O}\left(\gamma^{2}\right)$, we deduce

$$
\begin{aligned}
& \left\langle\mathscr{F}_{*}^{\prime}\left(\mathcal{W}\left(\bar{\gamma}_{n}\right)\right), \vartheta_{n}\right\rangle_{X^{*}, X}=\left\langle\mathscr{F}_{c_{*}+\bar{\gamma}_{n}}^{\prime}\left(\mathscr{W}\left(\bar{\gamma}_{n}\right)\right), \vartheta_{n}\right\rangle_{X^{*}, X}+\bar{\gamma}_{n}\left(\mathbb{B}^{\mathscr{W}}\left(\bar{\gamma}_{n}\right), \vartheta_{n}\right)_{H} \\
& =\left\langle\mathscr{F}_{c_{*}+\bar{\gamma}_{n}}^{\prime}\left(u_{c_{*}+\bar{\gamma}_{n}}\right)+\sigma\left(\bar{\gamma}_{n}\right) \mathbb{B} u_{c_{*}+\bar{\gamma}_{n}}, \vartheta_{n}\right\rangle_{X^{*}, X}+\bar{\gamma}_{n} \sigma\left(\bar{\gamma}_{n}\right)\left(u_{\left.c_{*}+\bar{\gamma}_{n}, \vartheta_{n}\right)_{H}}\right. \\
& =O\left(\bar{\gamma}_{n}^{2}\left\|\vartheta_{n}\right\|_{X}\right)+O\left(\left|\bar{\gamma}_{n}\right|^{3}\left\|\vartheta_{n}\right\|_{X}\right)=o\left(\left\|\vartheta_{n}\right\|_{X}^{7 / 3}\right)=o\left(\left\|\vartheta_{n}\right\|_{X}^{2}\right) \text {. }
\end{aligned}
$$


For the last line, we have used another Taylor expansion with $\mathscr{F}_{c_{*}+\bar{\gamma}_{n}}^{\prime}\left(u_{c_{*}+\bar{\gamma}_{n}}\right)=0$. Finally, Lemma 4.5 yields $\left\langle\mathscr{F}_{c_{*}+\bar{\gamma}_{n}}^{\prime \prime}\left(\vartheta_{c_{*}+\bar{\gamma}_{n}}\right) \vartheta_{n}, \vartheta_{n}\right\rangle_{X^{*}, X} \geq K_{0}\left\|\vartheta_{n}\right\|_{X}^{2}$. Reporting these expansions in (24) yields

$$
\mathscr{F}_{*}\left(u_{n}\right)-\mathscr{F}\left(\cup_{*}\right) \geq \frac{K_{0}}{4}\left\|\vartheta_{n}\right\|_{X}^{2}+o\left(\left\|\vartheta_{n}\right\|_{X}^{2}\right) \geq \frac{K_{0}}{8}\left\|\vartheta_{n}\right\|_{X}^{2}
$$

for $n$ sufficiently large, which contradicts our assumption.

We now need to find an extension of the functionals " $A$ " and " $P$ " used in [Maeda 2012] (and also in [Ohta 2011]). In these works, these functionals are built on what should be here " $J^{-1} \partial_{c} u_{c}=\mathbb{B} \partial_{x}^{-1} \partial_{c} \boldsymbol{u}_{c}$ ", but, unfortunately, $\partial_{c} \phi_{c}$ does not have vanishing integral over $\mathbb{R}$ (for instance, $\partial_{c} \eta_{c}$ has constant sign). We rely instead on a construction of a suitable approximation of " $J^{-1} \partial_{c}{U_{c}}_{c}$ ". A similar construction is used in [Lin 2002].

Lemma 4.9. For any $0<\kappa<1$, there exists a $\mathscr{b}^{2}$ mapping $\Upsilon_{\kappa}:\left(-\gamma_{1},+\gamma_{1}\right) \rightarrow X$ such that, for any $\gamma \in\left(-\gamma_{1},+\gamma_{1}\right), \Upsilon_{\kappa}(\gamma) \in H^{2} \times H^{1}$ is an odd function verifying

$$
\left\|J \Upsilon_{\kappa}(\gamma)-\left[\partial_{c} u_{c}\right]_{\mid c=c_{*}+\gamma}\right\|_{X} \leq \kappa
$$

Proof. We fix an even function $\Theta_{0} \in \mathscr{C}_{c}^{\infty}(\mathbb{R})$ such that $\int_{\mathbb{R}} \Theta_{0} d x=1$. For $T>0$ to be fixed later, but independent of $\gamma$ and $\kappa$, we set $t_{\kappa} \equiv T / \kappa^{2}>0$ and

$$
\Upsilon_{\kappa}(\gamma)(x) \equiv \mathbb{B} \int_{0}^{x}\left[\left[\partial_{c} u_{c}\right]_{\mid c=c_{*}+\gamma}(y)-\frac{1}{t_{\kappa}} \Theta_{0}\left(\frac{y}{t_{\kappa}}\right) \int_{\mathbb{R}}\left[\partial_{c} u_{c}\right]_{\mid c=c_{*}+\gamma}(z) d z\right] d y .
$$

It is clear that $\Upsilon_{\kappa}(\gamma) \in \mathscr{C}^{1}(\mathbb{R})$ and that, since $J=\partial_{x} \mathbb{B}$ and $\mathbb{B}^{2}=\operatorname{Id}_{2}$,

$$
J \Upsilon_{\kappa}(\gamma)-\left[\partial_{c} u_{c}\right]_{\mid c=c_{*}+\gamma}=\frac{1}{t_{\kappa}} \Theta_{0}\left(\frac{\cdot}{t_{\kappa}}\right) \int_{\mathbb{R}}\left[\partial_{c} u_{c}\right]_{\mid c=c_{*}+\gamma}(z) d z
$$

In particular,

$$
\left\|J \Upsilon_{\kappa}(\gamma)-\partial_{c}\left[u_{c}\right]_{\mid c=c_{*}+\gamma}\right\|_{X}^{2}=\left[\frac{1}{t_{\kappa}}\left\|\Theta_{0}\right\|_{L^{2}}^{2}+\frac{1}{t_{\kappa}^{3}}\left\|\partial_{x} \Theta_{0}\right\|_{L^{2}}^{2}\right]\left(\int_{\mathbb{R}}\left[\partial_{c} u_{c}\right]_{\mid c=c_{*}+\gamma}(z) d z\right)^{2} \leq \kappa^{2}
$$

if we choose $T=T\left(c_{*}, \cup_{*}, \Theta_{0}\right)>0$ sufficiently large and $\gamma_{1}$ smaller if necessary. Moreover, $\Upsilon_{\kappa}(\gamma)$ is odd since $u_{c}$ and $\Theta_{0}$ are even. In addition, the even function

$$
y \mapsto\left[\partial_{c} u_{c}\right]_{\mid c=c_{*}+\gamma}(y)-\frac{1}{t_{\kappa}} \Theta_{0}\left(\frac{y}{t_{\kappa}}\right) \int_{\mathbb{R}}\left[\partial_{c} u_{c}\right]_{\mid c=c_{*}+\gamma}(z) d z
$$

decays exponentially at infinity (since $\Theta_{0}$ has compact support and $\partial_{c} u_{c}$ decays exponentially), and has zero integral (since $\Theta_{0}$ has integral equal to one); hence

$$
\Upsilon_{\kappa}(\gamma)(x)=-\mathbb{B} \int_{x}^{+\infty}\left[\left[\partial_{c} u_{c}\right]_{\mid c=c_{*}+\gamma}(y)-\frac{1}{t_{\kappa}} \Theta_{0}\left(\frac{y}{t_{\kappa}}\right) \int_{\mathbb{R}}\left[\partial_{c} u_{c}\right]_{\mid c=c_{*}+\gamma}(z) d z\right] d y
$$


and decays exponentially at infinity. It follows easily from these two equalities that $\gamma \mapsto \Upsilon_{\kappa}(\gamma) \in L^{2} \times L^{2}$ is well-defined and continuous; hence also $\gamma \mapsto \Upsilon_{\kappa}(\gamma) \in H^{2} \times H^{1}$. By the same type of arguments,

$$
\frac{\partial \Upsilon_{\kappa}}{\partial \gamma}(\gamma)(x)=\mathbb{B} \int_{0}^{x}\left[\left[\partial_{c}^{2} u_{c}\right]_{\mid c=c_{*}+\gamma}(y)-\frac{1}{t_{\kappa}} \Theta_{0}\left(\frac{y}{t_{\kappa}}\right) \int_{\mathbb{R}}\left[\partial_{c}^{2} u_{c}\right]_{\mid c=c_{*}+\gamma}(z) d z\right] d y
$$

is well-defined and is a continuous function of $\gamma$ with values in $H^{2} \times H^{1}$, and similarly for the second derivative.

We now define, in the tubular neighborhood $O_{\varepsilon}$ of $\bigcup_{*}$, the functional (corresponding to " $A$ " in [Maeda 2012])

$$
\Omega_{\kappa}(U) \equiv\left(U(\cdot-\bar{y}(U)), \Upsilon_{\kappa}(\bar{\gamma}(u))\right)_{H}=\left(U, \Upsilon_{\kappa}(\bar{\gamma}(U))(\cdot+\bar{y}(u))\right)_{H}
$$

depending on $\kappa \in(0,1)$, which will be determined later. The first properties of $\Omega_{\kappa}$ are given below.

Lemma 4.10. For any $0<\kappa<1, \Omega_{\kappa}: \mathscr{O}_{\varepsilon} \rightarrow \mathbb{R}$ is of class $\mathscr{C}^{1}$. In addition, there exists some bounded mapping $\mathcal{N}_{\bar{\gamma}}: \mathscr{O}_{\varepsilon} \rightarrow X$ such that, if $\Psi_{\text {hy }} \in \mathscr{C}^{1}([0, T), X)$ is a solution to (15) that remains in $\mathscr{O}_{\varepsilon}$, then

$$
\frac{d}{d t} \Omega_{\kappa}\left(\Psi_{\mathrm{hy}}(t)\right)=\Xi_{\kappa}\left(\Psi_{\mathrm{hy}}(t)\right),
$$

where $\Xi_{\kappa}: \mathbb{O}_{\varepsilon} \rightarrow \mathbb{R}$ is defined by

$$
\Xi_{\kappa}(U) \equiv-\left\langle\mathscr{F}_{c_{*}+\bar{\gamma}(U)}^{\prime}(U),\left.\left\{J \Upsilon_{\kappa}(\bar{\gamma}(u))(\cdot+\bar{y}(u))+\left(U, \partial_{\gamma} \Upsilon_{\kappa}(\bar{\gamma}(u))(\cdot+\bar{y}(u))\right)_{H} \mathcal{N}_{\bar{\gamma}}(u)\right\}\right|_{X^{*}, X} \cdot\right.
$$

Proof. The fact that $\Omega_{\kappa}$ is of class $\mathscr{C}^{1}$ follows directly from the second expression and the fact that $\bar{y}$ and $\bar{\gamma}$ are $\mathscr{C}^{1}$ (in [Maeda 2012, formula (3.11)], the same remark as for the smoothness of $G$ after Lemma 4.3 holds, since it requires " $u \in D\left(T^{\prime}(0)\right)$ "). If $\Psi_{\text {hy }}=(\eta, u) \in \mathscr{C}^{1}([0, T), X)$ is a solution to (15) that remains in $\mathscr{O}_{\varepsilon}$, we therefore have, defining $\bar{\gamma}(t)=\bar{\gamma}\left(\Psi_{\mathrm{hy}}(t)\right)$ and $\bar{y}(t)=\bar{y}\left(\Psi_{\mathrm{hy}}(t)\right)$,

$$
\begin{aligned}
\frac{d}{d t} \Omega_{\kappa}\left(\Psi_{\text {hy }}(t)\right)=( & \left.\partial_{t} \Psi_{\text {hy }}(t), \Upsilon_{\kappa}(\bar{\gamma}(t))(\cdot+\bar{y}(t))\right)_{H} \\
& +\left(\Psi_{\text {hy }}(t), \partial_{x} \Upsilon_{\kappa}(\bar{\gamma}(t))(\cdot+\bar{y}(t))\right)_{H}\left\langle\bar{y}^{\prime}\left(\Psi_{\text {hy }}(t)\right), \partial_{t} \Psi_{\text {hy }}(t)\right\rangle_{X^{*}, X} \\
& +\left(\Psi_{\text {hy }}(t), \partial_{\gamma} \Upsilon_{\kappa}(\bar{\gamma}(t))(\cdot+\bar{y}(t))\right)_{H}\left\langle\bar{\gamma}^{\prime}\left(\Psi_{\text {hy }}(t)\right), \partial_{t} \Psi_{\text {hy }}(t)\right\rangle_{X^{*}, X}
\end{aligned}
$$

We now observe that the invariance of $\Omega_{\kappa}$ by translation provides by differentiation the equality, for $u \in \mathbb{O}_{\varepsilon}$,

$$
\begin{aligned}
& 0=\frac{d}{d y} \Omega_{\kappa}(u(\cdot-y))_{\mid y=0}=\left(u, \partial_{x} \Upsilon_{\kappa}(\bar{\gamma}(u))(\cdot+\bar{y}(u))\right)_{H} \\
& =\left(\mathbb{B} U, J \Upsilon_{\kappa}(\bar{\gamma}(U))(\cdot+\bar{y}(u))\right)_{H}=\left\langle P_{\text {hy }}^{\prime}(U), J \Upsilon_{\kappa}(\bar{\gamma}(U))(\cdot+\bar{y}(u))\right\rangle_{X^{*}, X} .
\end{aligned}
$$


In particular, the second term in (25) vanishes. In addition, since $\Psi_{\text {hy }}=(\eta, u) \in \mathscr{C}^{1}([0, T), X)$ is a solution to (15) that remains in $\sigma_{\varepsilon}$, we have, denoting by $\delta E_{\mathrm{hy}} / \delta \Psi$ the variational derivative,

$$
\begin{aligned}
& \left(\partial_{t} \Psi_{\mathrm{hy}}(t), \Upsilon_{\kappa}(\bar{\gamma}(t))(\cdot+\bar{y}(t))\right)_{H} \\
& =\left(J \frac{\delta E_{\mathrm{hy}}}{\delta \Psi_{\mathrm{hy}}}\left(\Psi_{\mathrm{hy}}(t)\right), \Upsilon_{\kappa}(\bar{\gamma}(t))(\cdot+\bar{y}(t))\right)_{H} \\
& =-\left(\frac{\delta E_{\mathrm{hy}}}{\delta \Psi_{\mathrm{hy}}}\left(\Psi_{\mathrm{hy}}(t)\right), J \Upsilon_{\kappa}(\bar{\gamma}(t))(\cdot+\bar{y}(t))\right)_{H}=-\left\langle E_{\mathrm{hy}}^{\prime}\left(\Psi_{\mathrm{hy}}(t)\right), J \Upsilon_{\kappa}(\bar{\gamma}(t))(\cdot+\bar{y}(t))\right\rangle_{X^{*}, X} \\
& =-\left\langle\mathscr{F}_{c_{*}}^{\prime}+\bar{\gamma}(t)\right. \\
& =-\left\langle\mathscr{F}_{c_{*}+\bar{\gamma}(t)}^{\prime}\left(\Psi_{\mathrm{hy}}(t)\right), J \Upsilon_{\kappa}(\bar{\gamma}(t))(\cdot+\bar{y}(t))\right\rangle_{X^{*}, X}-\left(c_{*}+\bar{\gamma}(t)\right)\left\langle P_{\mathrm{hy}}^{\prime}\left(\Psi_{\mathrm{hy}}(t)\right), J \Upsilon_{\kappa}(\bar{\gamma}(t))(\cdot++\bar{y}(t))\right\rangle_{X^{*}, X}
\end{aligned}
$$

by (26). In addition, since $\delta P_{\text {hy }} / \delta \Psi_{\text {hy }}=\mathbb{B} \Psi_{\text {hy }}$ and $J \mathbb{B}=\partial_{x}$,

$$
\begin{aligned}
\left\langle\bar{\gamma}^{\prime}\left(\Psi_{\mathrm{hy}}(t)\right), \partial_{t} \Psi_{\mathrm{hy}}(t)\right\rangle_{X^{*}, X} \\
=\left\langle\bar{\gamma}^{\prime}\left(\Psi_{\mathrm{hy}}(t)\right), J \frac{\delta E_{\mathrm{hy}}}{\delta \Psi_{\mathrm{hy}}}\left(\Psi_{\mathrm{hy}}(t)\right)\right\rangle_{X^{*}, X} \\
=\left\langle\bar{\gamma}^{\prime}\left(\Psi_{\mathrm{hy}}(t)\right), J \frac{\delta \mathscr{F}_{*}+\bar{\gamma}(u)}{\delta \Psi_{\mathrm{hy}}}\left(\Psi_{h} y(t)\right)\right\rangle_{X^{*}, X}+\left(c_{*}+\bar{\gamma}(u)\right)\left\langle\bar{\gamma}^{\prime}\left(\Psi_{\mathrm{hy}}(t)\right), \partial_{x} \Psi_{\mathrm{hy}}(t)\right\rangle_{X^{*}, X} .
\end{aligned}
$$

The second term vanishes since $\bar{\gamma}$ is invariant by translation (by definition; see the proof of Lemma 4.3). As a consequence,

$$
\begin{aligned}
& \left\langle\bar{\gamma}^{\prime}\left(\Psi_{\mathrm{hy}}(t)\right), \partial_{t} \Psi_{\mathrm{hy}}(t)\right\rangle_{X^{*}, X} \\
& \quad=\left(J \frac{\delta \mathscr{F}_{c_{*}+\bar{\gamma}(t)}}{\delta \Psi_{\mathrm{hy}}}\left(\Psi_{\mathrm{hy}}(t)\right), \square^{-1} \bar{\gamma}^{\prime}\left(\Psi_{\mathrm{hy}}(t)\right)\right)_{X}=-\left(\frac{\delta \mathscr{F}_{c_{*}+\bar{\gamma}(t)}}{\delta \Psi_{\mathrm{hy}}}\left(\Psi_{\mathrm{hy}}(t)\right), J^{-1} \bar{\gamma}^{\prime}\left(\Psi_{\mathrm{hy}}(t)\right)\right)_{X} \\
& \quad=-\left(\frac{\delta \mathscr{F}_{c_{*}+\bar{\gamma}(t)}}{\delta \Psi_{\mathrm{hy}}}\left(\Psi_{\mathrm{hy}}(t)\right), J^{-1} \bar{\gamma}^{\prime}\left(\Psi_{\mathrm{hy}}(t)\right)\right)_{H}-\left(\partial_{x} \frac{\delta \mathscr{F}_{c_{*}+\bar{\gamma}(t)}}{\delta \eta}\left(\Psi_{\mathrm{hy}}(t)\right), \partial_{x} J \square_{H^{1}}^{-1} \frac{\partial \bar{\gamma}}{\partial \eta}\left(\Psi_{\mathrm{hy}}(t)\right)\right)_{L^{2}}
\end{aligned}
$$

The first term is simply $-\left\langle\mathscr{F}_{c_{*}+\bar{\gamma}(U)}^{\prime}\left(\Psi_{\text {hy }}(t)\right), J^{-1} \bar{\gamma}^{\prime}\left(\Psi_{\text {hy }}(t)\right)\right\rangle_{X^{*}, X}$. We then define $\mathcal{N}_{\bar{\gamma}}: \mathcal{O}_{\varepsilon} \rightarrow X$ by $\mathcal{N}_{\bar{\gamma}}(u) \equiv J \square^{-1} \bar{\gamma}^{\prime}(u)-\left(\partial_{x}^{2} J_{H^{1}}^{-1}(\delta \bar{\gamma} / \delta \eta)(u), 0\right) \in X=H^{1} \times L^{2}$ (see the regularity shown for $\bar{\gamma}^{\prime}$ in Lemma 4.3), so that integration by parts yields

$$
\left\langle\bar{\gamma}^{\prime}\left(\Psi_{\mathrm{hy}}(t)\right), \partial_{t} \Psi_{\mathrm{hy}}(t)\right\rangle_{X^{*}, X}=-\left\langle\mathscr{F}_{c_{*}+\bar{\gamma}(u)}^{\prime}\left(\Psi_{\mathrm{hy}}(t)\right), \mathcal{N}_{\bar{\gamma}}\left(\Psi_{\mathrm{hy}}(t)\right)\right\rangle_{X^{*}, X}
$$

Inserting these relations into (25) then gives

$$
\begin{aligned}
& \frac{d}{d t} \Omega_{\kappa}\left(\Psi_{\text {hy }}(t)\right) \\
& =-\left\langle\mathscr{F}_{c_{*}+\bar{\gamma}(t)}^{\prime}\left(\Psi_{\text {hy }}(t)\right),\left\{J \Upsilon_{\kappa}(\bar{\gamma}(t))(\cdot+\bar{y}(t))+\left(\Psi_{\text {hy }}(t), \partial_{\gamma} \Upsilon_{\kappa}(\bar{\gamma}(t))(\cdot+\bar{y}(t))\right)_{H} \mathcal{N}_{\bar{\gamma}}\left(\Psi_{\text {hy }}(t)\right)\right\}\right\rangle_{X^{*}, X},
\end{aligned}
$$

which is the desired equality. 
If $\Psi_{\text {hy }} \in \mathscr{C}^{0}([0, T), X)$ is just a continuous in time solution to (15) that remains in $0_{\varepsilon}$, then the integrated relation

$$
\Omega_{\kappa}\left(\Psi_{\text {hy }}(t)\right)=\Omega_{\kappa}\left(\Psi_{\text {hy }}^{\text {in }}\right)+\int_{0}^{t} \Xi_{\kappa}\left(\Psi_{\text {hy }}(\tau)\right) d \tau
$$

holds, as can be seen by using the continuity of the flow and the approximation of such a solution by smoother ones (see [Gallo 2004]).

We now compute the asymptotics of $\Xi_{\kappa}(\mathcal{W}(\gamma))$ for $\gamma \rightarrow 0$ and small $\kappa$.

Lemma 4.11. We have

$$
\Xi_{\kappa}(W(\gamma))=-\frac{\gamma^{2} \ddot{P}_{*}}{2}+o_{(\gamma, \kappa) \rightarrow(0,0)}\left(\gamma^{2}\right) .
$$

Proof. The proof follows the one of Lemma 5 in [Maeda 2012]. As a first step, notice that $\bar{\gamma}(\mathcal{W}(\gamma))=\gamma$, $\bar{y}(\mathcal{W}(\gamma))=0$, as can be seen from the equality $G(\mathcal{W}(\gamma), 0,0,0)=0$ and the local uniqueness of the solution to $G=0$. Therefore, since $\mathscr{F}_{c_{*}+\gamma}^{\prime}\left(u_{c_{*}+\gamma}\right)=0$ and $\sigma(\gamma) \sim-\gamma^{2} \ddot{P}_{*} /\left(2\left\|u_{*}\right\|_{H}^{2}\right)$,

$$
\begin{aligned}
\mathscr{F}_{c_{*}+\bar{\gamma}(\mathscr{W}(\gamma))}^{\prime}(\mathcal{W}(\gamma)) & =\mathscr{F}_{c_{*}+\gamma}^{\prime}\left(u_{c_{*}+\gamma}+\sigma(\gamma) \mathbb{B} u_{c_{*}+\gamma}\right)=\sigma(\gamma) \mathscr{F}_{c_{*}+\gamma}^{\prime \prime}\left(\mathcal{U}_{c_{*}+\gamma}\right)\left[\mathbb{B} u_{c_{*}+\gamma}\right]+o_{\gamma \rightarrow 0}\left(\gamma^{2}\right) \\
& =-\frac{\gamma^{2} \ddot{P}_{*}}{2\left\|U_{*}\right\|_{H}^{2}} \mathscr{F}_{*}^{\prime \prime}\left(\mathcal{U}_{*}\right)\left[\mathbb{B} u_{*}\right]+o_{\gamma \rightarrow 0}\left(\gamma^{2}\right) .
\end{aligned}
$$

In addition, since $U_{c}$ is even and $\Upsilon_{\kappa}(\gamma)$ is odd, we deduce

$$
\left(\mathscr{W}(\gamma)(\cdot+\bar{y}(\mathcal{W}(\gamma))), \partial_{\gamma} \Upsilon_{\kappa}(\gamma)\right)_{H}=\left(u_{c_{*}+\gamma}+\sigma(\gamma) \mathbb{B} u_{c_{*}+\gamma}, \partial_{\gamma} \Upsilon_{\kappa}(\gamma)\right)_{H}=0
$$

Consequently,

$$
\Xi_{\kappa}(W(\gamma))=\frac{\gamma^{2} \ddot{P}_{*}}{2\left\|U_{*}\right\|_{H}^{2}}\left\langle\mathscr{F}_{*}^{\prime \prime}\left(\mathcal{W}_{*}\right)\left[\mathbb{B} u_{*}\right], J \Upsilon_{\kappa}(\gamma)\right\rangle_{X^{*}, X}+o_{\gamma \rightarrow 0}\left(\gamma^{2}\right),
$$

where " $o_{\gamma \rightarrow 0}\left(\gamma^{2}\right)$ " does not depend on $\kappa$. Moreover, by Lemma 4.9, $\left\|J \Upsilon_{\kappa}(\gamma)-\left[\partial_{c} u_{c}\right]_{\mid c=c_{*}+\gamma}\right\|_{X} \leq \kappa$ independently of $\gamma \in\left(-\gamma_{1},+\gamma_{1}\right)$; hence

$$
\begin{aligned}
\Xi_{\kappa}(\mathcal{W}(\gamma)) & =\frac{\gamma^{2} \ddot{P}_{*}}{2\left\|U_{*}\right\|_{H}^{2}}\left\langle\mathscr{F}_{*}^{\prime \prime}\left(u_{*}\right)\left[\mathbb{B} u_{*}\right],\left[\partial_{c} u_{c}\right]_{\mid c=c_{*}+\gamma}\right\rangle_{X^{*}, X}+o_{(\gamma, \kappa) \rightarrow(0,0)}\left(\gamma^{2}\right) \\
& =\frac{\gamma^{2} \ddot{P}_{*}}{2\left\|u_{*}\right\|_{H}^{2}}\left\langle\mathscr{F}_{*}^{\prime \prime}\left(u_{*}\right)\left[\mathbb{B} u_{*}\right],\left[\partial_{c} u_{c}\right]_{\mid c=c_{*}}\right\rangle_{X^{*}, X}+o_{(\gamma, \kappa) \rightarrow(0,0)}\left(\gamma^{2}\right) .
\end{aligned}
$$

Finally, using once again the equality (for $\phi \in X)\left\langle\mathscr{F}_{*}^{\prime \prime}\left(\mathcal{U}_{*}\right)\left[\partial_{c} u_{c}\right]_{\mid c=c_{*}}, \phi\right\rangle_{X^{*}, X}=\left(\mathbb{B} u_{*}, \phi\right)_{H}$ and that $\mathscr{F}_{*}^{\prime \prime}$ is self-adjoint, we infer

$$
\left\langle\mathscr{F}_{*}^{\prime \prime}\left(u_{*}\right)\left[\mathbb{B} u_{*}\right],\left[\partial_{c} u_{c}\right]_{\mid c=c_{*}}\right\rangle_{X^{*}, X}=\left\langle\mathscr{F}_{*}^{\prime \prime}\left(u_{*}\right)\left(\left[\partial_{c} u_{c}\right]_{\mid c=c_{*}}\right), \mathbb{B} u_{*}\right\rangle_{X^{*}, X}=\left\|\mathbb{B} u_{*}\right\|_{H}^{2}=\left\|u_{*}\right\|_{H}^{2},
$$

and reporting this into the previous expression gives the result.

We now compute the asymptotics of $\Xi_{\kappa}$ for more general functions. 
Lemma 4.12. Let $\varepsilon>0$ be small enough. If $u \in O_{\varepsilon}$ satisfies $P_{\mathrm{hy}}(u)=P_{\mathrm{hy}}\left(\mathcal{U}_{*}\right)$ and $\mathscr{F}_{*}\left(\mathcal{U}_{)}-\mathscr{F}_{*}\left(u_{*}\right)<0\right.$, then, we have

$$
\Xi_{\kappa}(u)=-\frac{\bar{\gamma}^{2}(u) \ddot{P}_{*}}{2}+o\left(\bar{\gamma}^{2}(u)\right)
$$

uniformly for $0<\kappa \leq|\bar{\gamma}(\mathcal{U})|^{3}$.

Proof. First, we may apply Lemma 4.8 and infer that $\|\vartheta(\mathcal{U})\|_{X}^{2}+|\alpha(\mathcal{U})|=\mathcal{O}\left(\left|\bar{\gamma}^{3}(U)\right|\right)$. We write $\Xi_{\kappa}(U)=$ $\Xi_{\kappa}(u(\cdot-\bar{y}(u)))=\Xi_{\kappa}\left(\mathcal{W}(\bar{\gamma}(u))+\vartheta(u)+\alpha(u) \mathbb{B} u_{c_{*}+\bar{\gamma}(u)}\right)=\Xi_{\kappa}(\mathcal{W}(\bar{\gamma}(u))+\vartheta(u))+\mathcal{O}\left(|\bar{\gamma}(u)|^{3}\right)$ and, recalling the expression

$$
\Xi_{\kappa}(u)=-\left\langle\mathscr{F}_{c_{*}+\bar{\gamma}(u)}^{\prime}(u),\left\{J \Upsilon_{\kappa}(\bar{\gamma}(u))(\cdot+\bar{y}(u))+\left(u, \partial_{\gamma} \Upsilon_{\kappa}(\bar{\gamma}(u))(\cdot+\bar{y}(u))\right)_{H} \mathcal{N}_{\bar{\gamma}}(u)\right\}\right\rangle_{X^{*}, X},
$$

we wish to make a Taylor expansion. First, note that

$$
\begin{aligned}
& \mathscr{F}_{c_{*}+\bar{\gamma}(u)}^{\prime}(\mathcal{U})=\mathscr{F}_{c_{*}+\bar{\gamma}(u)}^{\prime}(\mathcal{W}(\bar{\gamma}(u)))+\mathscr{F}_{c_{*}+\bar{\gamma}(u)}^{\prime \prime}(\mathcal{W}(\bar{\gamma}(u)))[\vartheta(u)]+\mathcal{O}\left(|\bar{\gamma}(u)|^{3}\right) \\
& =\mathscr{F}_{c_{*}+\bar{\gamma}(U)}^{\prime}(\mathcal{W}(\bar{\gamma}(u)))+\mathscr{F}_{c_{*}+\bar{\gamma}(u)}^{\prime \prime}\left(U_{c_{*}+\bar{\gamma}(U)}\right)[\vartheta(U)]+\mathcal{O}\left(|\bar{\gamma}(U)|^{3}\right) ;
\end{aligned}
$$

hence, since $\mathscr{F}_{c_{*}+\bar{\gamma}}^{\prime}\left(u_{c_{*}+\bar{\gamma}}\right)=0$ and (Lemma 4.1) $\sigma(\gamma)=\mathcal{O}\left(\gamma^{2}\right)$, we have $\mathscr{W}(\bar{\gamma})=U_{c_{*}+\bar{\gamma}}+\mathcal{O}\left(\gamma^{2}\right)$; thus

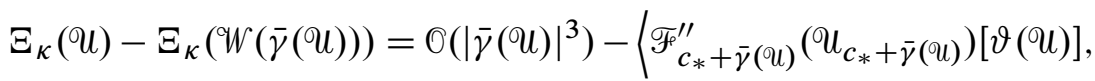

$$
\left.\left\{J \Upsilon_{\kappa}(\bar{\gamma}(u))(\cdot+\bar{y}(u))+\left(u, \partial_{\gamma} \Upsilon_{\kappa}(\bar{\gamma}(u))(\cdot+\bar{y}(u))\right)_{H} \mathcal{N}_{\bar{\gamma}}(u)\right\}\right|_{X^{*}, X} \cdot
$$

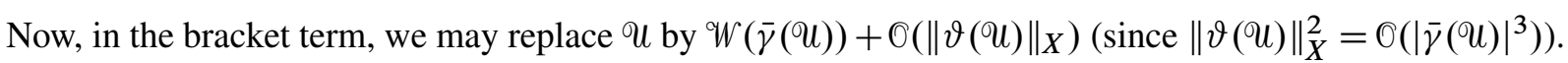
By the computations of Lemma 4.11 and the equalities $\bar{\gamma}(\mathcal{W}(\gamma))=\gamma, \bar{y}(\mathcal{W}(\gamma))=0$, this gives

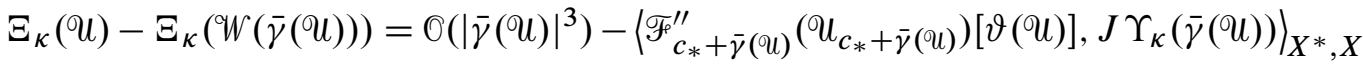

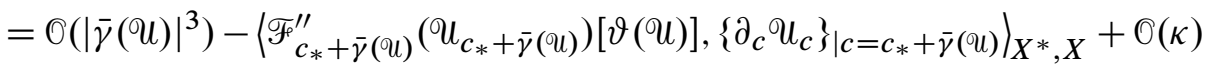

$$
\begin{aligned}
& =O\left(|\bar{\gamma}(u)|^{3}\right)-\left\langle\mathscr{F}_{c_{*}+\bar{\gamma}(u)}^{\prime \prime}\left(u_{c_{*}+\bar{\gamma}(u)}\right)\left[\left\{\partial_{c} u_{c}\right\}_{\mid c=c_{*}+\bar{\gamma}(u)}\right], \vartheta(u)\right\rangle_{X^{*}, X}+\mathcal{O}(\kappa)
\end{aligned}
$$

using Lemma 4.9 and the self-adjointness of $\mathscr{F}_{c_{*}+\bar{\gamma}(U)}^{\prime \prime}$. Choosing $0<\kappa \leq|\bar{\gamma}(\mathcal{U})|^{3}$ and from the equality $($ for $\phi \in X)\left\langle\mathscr{F}_{c}^{\prime \prime}\left(u_{c}\right)\left[\partial_{c} u_{c}\right], \phi\right\rangle_{X^{*}, X}=\left(\mathbb{B} u_{c}, \phi\right)_{H}$, we infer

$$
\Xi_{\kappa}(u)-\Xi_{\kappa}(w(\bar{\gamma}(u)))=\mathcal{O}\left(|\bar{\gamma}(u)|^{3}\right)-\left(\mathbb{B} u_{c_{*}+\bar{\gamma}(u)}, \vartheta(u)\right)_{H}=\mathscr{O}\left(|\bar{\gamma}(u)|^{3}\right),
$$

by the orthogonality condition in Lemma 4.5. Inserting the expansion of $\Xi_{\kappa}(\mathcal{W}(\gamma))$ given in Lemma 4.11 yields the conclusion.

Proof of Theorem 16. We have to show that there exists $\varepsilon>0$ such that, for any $\delta>0$, we can choose an initial datum at distance $\leq \delta$ from $\mathcal{U}_{*}$ but that escapes from $O_{\varepsilon}$. Since $\mathcal{W}(\gamma) \rightarrow \boldsymbol{U}_{*}$ in $X$, we shall take the initial datum to be $\mathcal{W}(\gamma)$ for some small $\gamma$, and denote by $\Psi_{\text {hy }}(t)$ the corresponding solution. In view of Lemma 4.2, we have $\mathscr{F}_{*}(\mathscr{W}(\gamma))-\mathscr{F}_{*}\left(U_{*}\right) \sim-\gamma^{3} \ddot{P}_{*} / 6$; hence we can choose $\gamma$ with the sign of $\ddot{P}_{*} \neq 0$ so that

$$
\mathscr{F}_{*}(\mathcal{W}(\gamma))-\mathscr{F}_{*}\left(\mathcal{U}_{*}\right) \sim-|\gamma|^{3}\left|\ddot{P}_{*}\right| / 6<0 \text {. }
$$


We now assume that $\Psi_{\text {hy }}(t)$ is globally defined and remains in $O_{\varepsilon}$, where $\varepsilon$ is as in Lemma 4.8. By conservation of energy and momentum and the construction of $\mathscr{W}(\gamma)$, we deduce $P_{\text {hy }}\left(\Psi_{\text {hy }}(t)\right)=P_{\text {hy }}(\mathscr{W}(\gamma))=$ $P_{\text {hy }}\left(U_{*}\right)$, and $\mathscr{F}_{*}\left(\Psi_{\text {hy }}(t)\right)-\mathscr{F}_{*}\left(U_{*}\right)=\mathscr{F}_{*}(\mathscr{W}(\gamma))-\mathscr{F}_{*}\left(U_{*}\right)<0$. The first step is to have a control on $\bar{\gamma}(t) \equiv \bar{\gamma}\left(\Psi_{\text {hy }}(t)\right)$. We define $\alpha(t)=\alpha\left(\Psi_{\text {hy }}(t)\right), \bar{y}(t)=\bar{y}\left(\Psi_{\text {hy }}(t)\right)$ and $\vartheta(t)=\vartheta\left(\Psi_{\text {hy }}(t)\right)$. Applying Lemma 4.8, we obtain $\|\vartheta(t)\|_{X}^{2}+|\alpha(t)|=\mathcal{O}\left(\left|\bar{\gamma}^{3}(t)\right|\right)$. In addition, Lemma 4.2 and a Taylor expansion give

$$
\begin{aligned}
& \mathscr{F}_{*}\left(\Psi_{\text {hy }}(t)\right)-\mathscr{F}_{*}\left(U_{*}\right) \\
& =\mathscr{F}_{*}\left(W(\bar{\gamma}(t))+\vartheta(t)+\alpha(t) \mathbb{B} u_{c_{*}+\bar{\gamma}(t)}\right)-\mathscr{F}_{*}\left(U_{*}\right) \\
& =\mathscr{F}_{*}(\mathscr{W}(\bar{\gamma}(t)))-\mathscr{F}_{*}\left(U_{*}\right)+\left\langle\mathscr{F}_{*}^{\prime}(\mathscr{W}(\bar{\gamma}(t))), \vartheta(t)\right\rangle_{X^{*}, X}+\frac{1}{2}\left\langle\mathscr{F}_{*}^{\prime \prime}(\mathscr{W}(\bar{\gamma}(t))) \vartheta(t), \vartheta(t)\right\rangle_{X^{*}, X}+o\left(\left|\bar{\gamma}^{3}(t)\right|\right) \\
& =-\frac{\bar{\gamma}^{3}(t) \ddot{P}_{*}}{6}+\left\langle\mathscr{F}_{*}^{\prime}(\mathscr{W}(\bar{\gamma}(t))), \vartheta(t)\right\rangle_{X^{*}, X}+\frac{1}{2}\left\langle\mathscr{F}_{*}^{\prime \prime}(\mathscr{W}(\bar{\gamma}(t))) \vartheta(t), \vartheta(t)\right\rangle_{X^{*}, X}+o\left(\left|\bar{\gamma}^{3}(t)\right|\right),
\end{aligned}
$$

where we have used that $\mathscr{F}_{*}^{\prime}(\mathscr{W}(\bar{\gamma}(t))=o(1)$ (for the terms involving $\alpha(t))$ and Lemma 4.2. Furthermore, by the orthogonality relations in Lemma 4.3 and using that $\sigma(\gamma)=\mathcal{O}\left(\gamma^{2}\right)$ and $\mathscr{F}_{c}^{\prime}\left(\vartheta_{c}\right)=0$, we have

$$
\begin{aligned}
\left\langle\mathscr{F}_{*}^{\prime}(\mathcal{W}(\bar{\gamma}(t))), \vartheta(t)\right\rangle_{X^{*}, X} & \left.=\left\langle\mathscr{F}_{c_{*}+\bar{\gamma}(t)}^{\prime}(\mathcal{W}(\bar{\gamma}(t))), \vartheta(t)\right\rangle_{X^{*}, X}+\bar{\gamma}(t)\left(\mathbb{B}^{\mathcal{W}}(\bar{\gamma}(t))\right), \vartheta(t)\right)_{H} \\
& =\left\langle\mathscr{F}_{c_{*}+\bar{\gamma}(t)}^{\prime}\left(U_{c_{*}+\bar{\gamma}(t)}+\sigma(\bar{\gamma}(t))\right) \mathbb{B} u_{c_{*}+\bar{\gamma}(t)}, \vartheta(t)\right\rangle_{X^{*}, X}=\mathcal{O}\left(\left|\bar{\gamma}^{7 / 2}(t)\right|\right) .
\end{aligned}
$$

In addition, by Lemma 4.5 , the second-to-last term is $\geq K_{0}\|\vartheta(t)\|_{X}^{2} / 2$. As a consequence, by conservation of $\mathscr{F}_{*}\left(\Psi_{\text {hy }}(t)\right)$, we infer, for small $\gamma$,

$$
0>-|\gamma|^{3}\left|\ddot{P}_{*}\right| / 3>\mathscr{F}_{*}(\mathscr{W}(\gamma))-\mathscr{F}_{*}\left(\cup_{*}\right)=\mathscr{F}_{*}\left(\Psi_{\mathrm{hy}}(t)\right)-\mathscr{F}_{*}\left(\cup_{*}\right) \geq-\frac{\bar{\gamma}^{3}(t) \ddot{P}_{*}}{6}+o\left(\left|\bar{\gamma}^{3}(t)\right|\right),
$$

In particular, this forces $\bar{\gamma}(t)$ to always be of the sign of $\ddot{P}_{*}$ and to satisfy $|\bar{\gamma}(t)| \geq|\gamma| / 2$ (provided $\varepsilon$ and $\gamma$ are small enough).

Since, now, we have a good upper bound for $|\bar{\gamma}(t)|$, we can choose $\kappa=\kappa(\gamma) \equiv \gamma^{3} / 8$, which is such that, for any $t \geq 0, \kappa \leq|\bar{\gamma}(t)|^{3}$. In particular, we can apply Lemma 4.12 and get

$$
\Xi_{\kappa}\left(\Psi_{\text {hy }}(t)\right)=-\frac{\bar{\gamma}(t)^{2} \ddot{P}_{*}}{2}+o\left(\bar{\gamma}(t)^{2}\right)
$$

With this choice $\kappa=\kappa(\gamma)$, we deduce from Lemma 4.10 that

$$
\frac{d}{d t} \Omega_{\kappa(\gamma)}\left(\Psi_{\mathrm{hy}}(t)\right)=\Xi_{\kappa}\left(\Psi_{\mathrm{hy}}(t)\right)=-\frac{\bar{\gamma}(t)^{2} \ddot{P}_{*}}{2}+o\left(\bar{\gamma}(t)^{2}\right) .
$$

Since $|\bar{\gamma}(t)| \geq|\gamma| / 2$, it follows that, when $\ddot{P}_{*}<0$ (the case $\ddot{P}_{*}>0$ is analogous),

$$
\frac{d}{d t} \Omega_{\kappa(\gamma)}\left(\Psi_{\text {hy }}(t)\right) \geq-\frac{\gamma^{2} \ddot{P}_{*}}{8}>0 ;
$$

hence $\Omega_{\kappa(\gamma)}\left(\Psi_{\text {hy }}(t)\right)$ is unbounded as $t$ goes to $+\infty$. However, by definition of $\Omega_{\kappa}$, we have by the Cauchy-Schwarz inequality $\left|\Omega_{\kappa(\gamma)}(U)\right| \leq\|u\|_{H}\left\|\Upsilon_{\kappa(\gamma)}\right\|_{H} \leq C(\gamma)$ for $U \in O_{\varepsilon}$. We have reached a contradiction. The proof of Theorem 16 is complete. 


\section{The linear instability $\left(0<c_{*}<\mathfrak{c}_{s}\right)$}

5A. Proof of Theorem 13. Existence of at least one unstable eigenvalue. The proof of the existence of at least one unstable eigenvalue relies on the Evans function technique, as in [Zumbrun 2008; BenzoniGavage 2010b]. We shall actually use Theorem 1 in [Benzoni-Gavage 2010b] when observing (see, e.g., [Benzoni-Gavage 2010a]) that the Euler-Korteweg system

$$
\left\{\begin{array}{l}
\partial_{t} \rho+2 \partial_{x}(\rho u)=0 \\
\partial_{t} u+2 u \partial_{x} u-\partial_{x}(f(\rho))-\partial_{x}\left(K(\rho) \partial_{x}^{2} \rho+\frac{1}{2} K^{\prime}(\rho)\left(\partial_{x} \rho\right)^{2}\right)=0
\end{array}\right.
$$

where $K:(0,+\infty) \rightarrow(0,+\infty)$ is the (smooth enough) capillarity, reduces to (2) (where, we recall, $\Psi=A \mathrm{e}^{i \phi}, \rho=A^{2}$ and $\left.u=\partial_{x} \phi\right)$; namely,

$$
\left\{\begin{array}{l}
\partial_{t} \rho+2 \partial_{x}(\rho u)=0 \\
\partial_{t} u+2 u \partial_{x} u-\partial_{x}(f(\rho))-\partial_{x}\left(\frac{\partial_{x}^{2}(\sqrt{\rho})}{\sqrt{\rho}}\right)=0
\end{array}\right.
$$

for the capillarity $K(\varrho)=1 /(2 \varrho)$, as can be shown by straightforward computations. The associated eigenvalue problem in the moving frame is

$$
\left\{\begin{array}{l}
\lambda \zeta-c_{*} \partial_{x} \zeta+2 \partial_{x}\left(\left(r_{0}^{2}+\eta_{*}\right) v+\zeta u_{*}\right)=0, \\
\lambda v-c_{*} \partial_{x} v+2 \partial_{x}\left(u_{*} v\right)-\partial_{x}\left(f^{\prime}\left(r_{0}^{2}+\eta_{*}\right) \zeta\right) \\
\quad-\partial_{x}\left\{\frac{1}{2 \sqrt{r_{0}^{2}+\eta_{*}}} \partial_{x}^{2}\left(\frac{\zeta}{\sqrt{r_{0}^{2}+\eta_{*}}}\right)-\frac{\zeta \partial_{x}^{2}\left(\sqrt{r_{0}^{2}+\eta_{*}}\right)}{2\left(r_{0}^{2}+\eta_{*}\right)^{3 / 2}}\right\}=0 .
\end{array}\right.
$$

The link with the original eigenvalue problem (6) is done through the formula

$$
w=U_{*}\left(\frac{\zeta}{2}+i \int_{-\infty}^{x} v\right)
$$

since this corresponds to

$$
\Psi=U_{c_{*}}+\psi=U_{c_{*}}+\mathrm{e}^{\lambda t} w(x)=\left(A_{c_{*}}+\mathrm{e}^{\lambda t} \zeta(x)\right) \exp \left(i \phi_{c_{*}}+i \mathrm{e}^{\lambda t} \int_{-\infty}^{x} v\right) .
$$

Notice indeed that the second equation in (27) gives $\int_{\mathbb{R}} v d x=0$. It then follows from Theorem 1 in [Benzoni-Gavage 2010b] that, under the assumption $\left(d P\left(U_{c}\right) / d c\right)_{\mid c=c_{*}}>0$, there exists at least one unstable eigenvalue $\gamma_{0} \in(0,+\infty)$.

Existence of at most one unstable eigenvalue. The fact that there exists at most one unstable eigenvalue follows from arguments as in [Benzoni-Gavage et al. 2005, Appendix B] and is a direct consequence of Theorem 3.1 in [Pego and Weinstein 1992], that we recall now.

Theorem 25 [Pego and Weinstein 1992]. Let $\mathscr{F}$ and $\mathscr{L}$ be two operators on a real Hilbert space $X$, with $\mathscr{L}$ self-adjoint and $\mathscr{S}$ skew-symmetric. Then, the number of eigenvalues, counting algebraic multiplicities, of $[\mathscr{L} \mathscr{L}]_{\mathbb{C}}$ in the right half-plane $\{\operatorname{Re}>0\}$ is less than or equal to the number of negative eigenvalues of $\mathscr{L}$, counting multiplicities. 
In order to apply this result to our problem, let us write the eigenvalue problem (27) under the form

$$
\lambda\left(\begin{array}{l}
\zeta \\
v
\end{array}\right)=-\partial_{x}\left(\begin{array}{ll}
0 & 1 \\
1 & 0
\end{array}\right) \mathscr{L}\left(\begin{array}{l}
\zeta \\
v
\end{array}\right)
$$

where $\mathcal{M}$ is the self-adjoint Sturm-Liouville operator

$$
\mu \equiv-f^{\prime}\left(r_{0}^{2}+\eta_{*}\right)-\frac{1}{2 \sqrt{r_{0}^{2}+\eta_{*}}} \partial_{x}^{2}\left(\frac{\cdot}{\sqrt{r_{0}^{2}+\eta_{*}}}\right)+\frac{\partial_{x}^{2}\left(\sqrt{r_{0}^{2}+\eta_{*}}\right)}{2\left(r_{0}^{2}+\eta_{*}\right)^{3 / 2}}
$$

(which is bounded from below) on $\mathscr{H} \equiv L^{2} \times L^{2}$ and with

$$
\mathscr{L} \equiv\left(\begin{array}{cc}
M & 2 u_{*}-c_{*} \\
2 u_{*}-c_{*} & 2\left(r_{0}^{2}+\eta_{*}\right)
\end{array}\right) .
$$

We are in the setting of Theorem 25 with $\mathscr{F}=-\partial_{x}\left(\begin{array}{ll}0 & 1 \\ 1 & 0\end{array}\right)$ skew-symmetric and $\mathscr{L}$ self-adjoint. We thus show that $\mathscr{L}$ has at most one negative eigenvalue. Since $r_{0}^{2}+\eta_{*}$ remains bounded away from zero, it is clear that, for $\sigma<0$ and $(\zeta, v)$ given, $\mathscr{L}(\zeta, v)^{t}=\sigma(\zeta, v)^{t}$ if and only if

$$
\mu^{\dagger} \zeta-\frac{\left(c_{*}-2 u_{*}\right)^{2}}{2\left(r_{0}^{2}+\eta_{*}\right)} \cdot \frac{\sigma}{2\left(r_{0}^{2}+\eta_{*}\right)-\sigma} \zeta=\sigma \zeta, \quad \text { with } \mu^{\dagger} \equiv \mathcal{M}-\frac{\left(c_{*}-2 u_{*}\right)^{2}}{2\left(r_{0}^{2}+\eta_{*}\right)},
$$

since we may express $v$ in terms of $\zeta$ with the second equation. We observe that the translation invariance shows that $\partial_{x}\left(\eta_{*}, u_{*}\right)^{t}$ belongs to the kernel of $\mathscr{L}$; that is, using once again the relation $2 u_{c}=2 \partial_{x} \phi_{c}=c \eta_{c} /\left(\eta_{c}+r_{0}^{2}\right), \mu^{\dagger} \partial_{x} \eta_{*}=0$. Furthermore, $\mu^{\dagger}$ has the same continuous spectrum as its constant coefficient limit as $x \rightarrow \pm \infty$, namely

$$
-\frac{1}{2 r_{0}^{2}} \partial_{x}^{2}+\frac{\mathfrak{c}_{s}^{2}-c_{*}^{2}}{2 r_{0}^{2}}
$$

that is, $\sigma_{\text {ess }}\left(\mathcal{M}^{\dagger}\right)=\left[\mathfrak{c}_{s}^{2}-c_{*}^{2},+\infty\right) \subset(0,+\infty)$, since $0<c_{*}<\mathfrak{c}_{s}$. Since $\partial_{x} \eta_{*}$ has exactly one zero (at $x=0$ ), it follows from standard Sturm-Liouville theory that $\mathcal{M}^{\dagger}$ has precisely one negative eigenvalue $\mu<0$ and that the second eigenvalue is 0 . Taking the scalar product with (29) yields

$$
\left\langle\mathcal{M}^{\dagger} \zeta, \zeta\right\rangle_{L^{2}}-\int_{\mathbb{R}} \frac{\sigma\left(c_{*}-2 u_{*}\right)^{2} \zeta^{2}}{2\left(r_{0}^{2}+\eta_{*}\right)\left[2\left(r_{0}^{2}+\eta_{*}\right)-\sigma\right]} d x=\sigma\|\zeta\|_{L^{2}}^{2}
$$

Now, for $s \leq 0$, we consider the self-adjoint operator

$$
\mathcal{M}_{s}^{\dagger} \equiv \mathcal{M}^{\dagger}-\frac{\left(c_{*}-2 u_{*}\right)^{2}}{2\left(r_{0}^{2}+\eta_{*}\right)} \cdot \frac{s}{2\left(r_{0}^{2}+\eta_{*}\right)-s} .
$$

Clearly, $\mu_{s=0}^{\dagger}=\mathcal{M}^{\dagger}, \sigma_{\mathrm{ess}}\left(\mathcal{M}_{s}^{\dagger}\right) \subset\left[\mathfrak{c}_{s}^{2}-c_{*}^{2},+\infty\right) \subset(0,+\infty)$, and $\mathbb{R}_{-} \ni s \mapsto \mathcal{M}_{s}^{\dagger}$ is decreasing. Let us assume now that the self-adjoint operator $\mathscr{L}$ has at least two negative eigenvalues. Then, we denote by $\sigma_{1}<\sigma_{2}<0$ the two smallest eigenvalues of $\mathscr{L}$ (necessarily simple), and $\zeta_{1}, \zeta_{2}$ two associated eigenvectors. Since $\mathscr{L}$ is self-adjoint, $\left\langle\zeta_{1}, \zeta_{2}\right\rangle_{L^{2}}=0$. Furthermore, $\left\langle\mathcal{M}_{s=\sigma_{1}}^{\dagger} \zeta_{1}, \zeta_{1}\right\rangle_{L^{2}}=\sigma_{1}\left\|\zeta_{2}\right\|_{L^{2}}^{2}<0$; hence, by monotonicity, $\left\langle\mu_{s}^{\dagger} \zeta_{2}, \zeta_{2}\right\rangle_{L^{2}}<0$ for any $\sigma_{1} \leq s \leq 0$. Therefore, $\mu_{s}^{\dagger}$ has at least one negative eigenvalue for $\sigma_{1} \leq s \leq 0$. We denote by $\lambda_{\min }(s)$ the smallest eigenvalue of $\mathcal{M}_{s}^{\dagger}$. Then, $\lambda_{\min }(s=0)=\mu<0$ 
and $\lambda_{\text {min }}$ decreases in $\left[\sigma_{1}, 0\right]$. Moreover, we may choose a positive eigenvector $\zeta_{s}$ for the eigenvalue $\lambda_{1}(s)$, with $\zeta_{1}=\zeta_{\sigma_{1}}$. Since $\sigma\left(\mathcal{M}^{\dagger}\right) \cap \mathbb{R}_{+}=\{\mu, 0\}$, it follows from the monotonicity that, for any $\sigma_{1} \leq s<0$, we have $\sigma\left(M_{s}^{\dagger}\right) \cap \mathbb{R}_{-}=\left\{\lambda_{\min }(s)\right\}$. When $s=\sigma_{2} \in\left(\sigma_{1}, 0\right)$, we then have $\sigma_{2} \in \sigma\left(M_{s=\sigma_{2}}^{\dagger} \cap \mathbb{R}_{-}\right.$, and thus $\sigma_{2}=\lambda_{\min }\left(\sigma_{2}\right)$, which implies that we may choose $\zeta_{2}>0$ without loss of generality. Similarly, if $s=\sigma_{2}$, we see that we may choose $\zeta_{2}>0$. We obtain a contradiction since then $\left\langle\zeta_{1}, \zeta_{2}\right\rangle_{L^{2}}>0$ and thus $\zeta_{1}$ and $\zeta_{2}$ cannot be orthogonal in $L^{2}$. We have thus shown that $\mathscr{L}$ has at most one negative eigenvalue, and then Theorem 25 shows that $\mathscr{L} \mathscr{L}$ has at most one eigenvalue in $\{\operatorname{Re}>0\}$, as wished.

5B. Resolvent and semigroup estimates (proof of Corollary 15). In this section, we drop the "*" for the traveling wave we are considering. When linearizing the NLS equation in the moving frame with speed $c$, we obtain

or

$$
i \frac{\partial \psi}{\partial t}-i c \partial_{x} \psi+\partial_{x}^{2} \psi+\psi f\left(|U|^{2}\right)+2\langle\psi, U\rangle f^{\prime}\left(|U|^{2}\right) U=0,
$$

$$
\begin{aligned}
\frac{\partial}{\partial t}\left(\begin{array}{l}
\psi_{1} \\
\psi_{2}
\end{array}\right) & =\left(\begin{array}{cc}
c \partial_{x}-2 f^{\prime}\left(|U|^{2}\right) U_{1} U_{2} & -\partial_{x}^{2}-f\left(|U|^{2}\right)-2 f^{\prime}\left(|U|^{2}\right) U_{2}^{2} \\
\partial_{x}^{2}+f\left(|U|^{2}\right)+2 f^{\prime}\left(|U|^{2}\right) U_{1}^{2} & c \partial_{x}+2 f^{\prime}\left(|U|^{2}\right) U_{1} U_{2}
\end{array}\right)\left(\begin{array}{l}
\psi_{1} \\
\psi_{2}
\end{array}\right) \\
& =\left(\begin{array}{cc}
0 & 1 \\
-1 & 0
\end{array}\right)\left(\begin{array}{cc}
-\partial_{x}^{2}-f\left(|U|^{2}\right)-2 f^{\prime}\left(|U|^{2}\right) U_{1}^{2} & -c \partial_{x}-2 f^{\prime}\left(|U|^{2}\right) U_{1} U_{2} \\
c \partial_{x}-2 f^{\prime}\left(|U|^{2}\right) U_{1} U_{2} & -\partial_{x}^{2}-f\left(|U|^{2}\right)-2 f^{\prime}\left(|U|^{2}\right) U_{2}^{2}
\end{array}\right)\left(\begin{array}{l}
\psi_{1} \\
\psi_{2}
\end{array}\right) .
\end{aligned}
$$

We wish to show that this linear equation can be solved using a continuous semigroup. In order to handle later the nonlinear terms, we work in $H^{1}\left(\mathbb{R}, \mathbb{C}^{2}\right)$ instead of $L^{2}\left(\mathbb{R}, \mathbb{C}^{2}\right)$. Therefore, we consider the unbounded operator $\mathscr{A}: D(\mathscr{A})=H^{3}\left(\mathbb{R}, \mathbb{C}^{2}\right) \subset H^{1}\left(\mathbb{R}, \mathbb{C}^{2}\right) \rightarrow H^{1}\left(\mathbb{R}, \mathbb{C}^{2}\right)$ on $H^{1}\left(\mathbb{R}, \mathbb{C}^{2}\right)$ defined by

$$
\mathscr{A} \equiv\left(\begin{array}{cc}
c \partial_{x}-2 f^{\prime}\left(|U|^{2}\right) U_{1} U_{2} & -\partial_{x}^{2}-f\left(|U|^{2}\right)-2 f^{\prime}\left(|U|^{2}\right) U_{2}^{2} \\
\partial_{x}^{2}+f\left(|U|^{2}\right)+2 f^{\prime}\left(|U|^{2}\right) U_{1}^{2} & c \partial_{x}+2 f^{\prime}\left(|U|^{2}\right) U_{1} U_{2}
\end{array}\right) .
$$

It follows easily that, for $\psi=\left(\begin{array}{l}\psi_{1} \\ \psi_{2}\end{array}\right) \in H^{1}\left(\mathbb{R}, \mathbb{C}^{2}\right)$,

$$
\begin{aligned}
& \operatorname{Re}\left(\langle\mathscr{A} \psi \mid \psi\rangle_{H^{1}\left(\mathbb{R}, \mathbb{C}^{2}\right)}\right) \\
& =\operatorname{Re}\left(\left\langle-2 f^{\prime}\left(|U|^{2}\right) U_{1} U_{2} \psi_{1}, \psi_{1}\right\rangle_{H^{1}(\mathbb{R}, \mathbb{C})}+\left\langle-2 f^{\prime}\left(|U|^{2}\right) U_{1} U_{2} \psi_{2}, \psi_{2}\right\rangle_{H^{1}(\mathbb{R}, \mathbb{C})}\right. \\
& \left.\quad+\left\langle\left[f\left(|U|^{2}\right)+2 f^{\prime}\left(|U|^{2}\right) U_{1}^{2}\right] \psi_{1}, \psi_{2}\right\rangle_{H^{1}(\mathbb{R}, \mathbb{C})}-\left\langle\left[f\left(|U|^{2}\right)+2 f^{\prime}\left(\mid U^{2}\right) U_{1}^{2}\right] \psi_{2}, \psi_{1}\right\rangle_{H^{1}(\mathbb{R}, \mathbb{C})}\right) \\
& \leq K\|\psi\|_{H^{1}\left(\mathbb{R}, \mathbb{C}^{2}\right)}^{2} .
\end{aligned}
$$

Moreover, the spectrum of $\mathscr{A}$ is included in the half-space $\left\{\operatorname{Re} \leq \sigma_{0}\right\}$; hence $\mathscr{A}$ generates a continuous semigroup $\mathrm{e}^{t \AA A}$ on $H^{1}\left(\mathbb{R}, \mathbb{C}^{2}\right)$.

In order to estimate the growth of the semigroup $e^{t \mathscr{A}}$ on $H^{1}\left(\mathbb{R}, \mathbb{C}^{2}\right)$, we could try to use the same approach as [Di Menza and Gallo 2007], which relies on the proof of the spectral mapping theorem in [Gesztesy et al. 2000]. However, our situation is slightly different since, in these studies, the reference solution is real-valued (it is a bound state in [Gesztesy et al. 2000] and the kink in [Di Menza and Gallo 2007]). Therefore, $U_{2}=0$ and $\mathscr{A}$ has no diagonal term, and the system is much more decoupled than in our situation. As a matter of fact, it is not very clear whether the arguments of [Gesztesy et al. 2000] carry over to our problem. We thus have chosen to use the general approach given in Appendix B. We 
thus verify the assumptions of Theorem B.5 (see also Corollary B.6) there, which are easy: $\mathscr{A}$ generates a semigroup in $H^{1}\left(\mathbb{R}, \mathbb{C}^{2}\right)$ and the spectrum of $\mathscr{A}$ is of the form $i \mathbb{R} \cup\left\{-\gamma_{0},+\gamma_{0}\right\}$, where $i \mathbb{R}$ is the essential spectrum and $\pm \gamma_{0}$ two simple eigenvalues. Moreover, the eigenvector associated with $\gamma_{0}$ belongs to $H^{3}\left(\mathbb{R}, \mathbb{C}^{2}\right)=D(J)$. Therefore, Theorem B.5 in Appendix B applies and the growth estimate for the linearized problem follows. For the nonlinear instability result, we argue as for Corollary B.6 in Appendix B, since the manifold $\mathfrak{M}=\left\{\left|U_{*}\right|(\cdot-y), y \in \mathbb{R}\right\}$ is transverse to the curve $\sigma \mapsto\left|U_{*}+\sigma w\right|$ in $r_{0}+H^{1}(\mathbb{R})$. Indeed, it follows from (28) that $\left|U_{*}+\sigma w\right|=A_{*}+\sigma \zeta+\mathscr{O}_{H^{1}}\left(\sigma^{2}\right)$. Assume that $\zeta=\alpha \partial_{x}\left|U_{*}\right|$, with $\alpha \in \mathbb{R}$. Then, integration of the first equation of (27) provides

$$
\lambda\left(\left|U_{*}\right|-r_{0}\right)-c_{*} \partial_{x}\left|U_{*}\right|+2\left(\left(r_{0}^{2}+\eta_{*}\right) v+u_{*} \zeta\right)=0
$$

hence, using that $\left|U_{*}\right|=\sqrt{r_{0}^{2}+\eta_{*}}$ and the equality $2 u_{*}=c \eta_{*} /\left(r_{0}^{2}+\eta_{*}\right)$, we infer

$$
v+\alpha\left\{\lambda \frac{\left|U_{*}\right|-r_{0}}{r_{0}^{2}+\eta_{*}}+\frac{c_{*} r_{0}^{2}}{4\left(r_{0}^{2}+\eta_{*}\right)^{3 / 2}} \partial_{x} \eta_{*}\right\}=0
$$

Since $\int_{\mathbb{R}} v=0$ and $\left|U_{*}\right|-r_{0}$ has constant sign in $\mathbb{R}$, integrating over $\mathbb{R}$ then implies $\alpha=0$, which in turn yields $\zeta=v=0$ and $w_{*}=0$, a contradiction. Consequently, $\zeta \notin \mathbb{R} \partial_{x}\left|U_{*}\right|$ and the manifold $\mathfrak{M}=\left\{\left|U_{*}\right|(\cdot-y), y \in \mathbb{R}\right\}$ is indeed transverse to the curve $\sigma \mapsto\left|U_{*}+\sigma w\right|$ in $r_{0}+H^{1}(\mathbb{R})$.

\section{Stability analysis for the kink $(c=0)$}

6A. Proof of Lemma 20. Let us recall that the momentum $P\left(U_{c}\right)$, for $c>0$, has the expression

$$
P\left(U_{c}\right)=c \int_{\xi_{c}}^{0} \frac{\xi^{2}}{r_{0}^{2}+\xi} \frac{d \xi}{\sqrt{-V_{c}(\xi)}}
$$

since $\operatorname{sgn}\left(\xi_{c}\right)=-1$. Therefore, we decompose $P\left(U_{c}\right)$ with two integrals:

$$
P\left(U_{c}\right)=c \int_{\xi_{c}}^{0} \frac{\xi^{2}}{r_{0}^{2}+\xi} \frac{d \xi}{\sqrt{-\mathscr{V}_{c}^{\prime}\left(\xi_{c}\right)\left(\xi-\xi_{c}\right)}}+c \int_{\xi_{c}}^{0} \frac{\xi^{2}}{r_{0}^{2}+\xi}\left(\frac{1}{\sqrt{-\mathscr{V}_{c}(\xi)}}-\frac{1}{\sqrt{-\mathscr{V}_{c}^{\prime}\left(\xi_{c}\right)\left(\xi-\xi_{c}\right)}}\right) d \xi
$$

Using the change of variables $\xi=t \xi_{c}$, the second integral in (31) is equal to

$$
\begin{aligned}
\xi_{c}^{3} \int_{1}^{0} \frac{t^{2}}{r_{0}^{2}+t \xi_{c}}\left(\frac{1}{\sqrt{-\mathscr{V}_{c}\left(t \xi_{c}\right)}}-\frac{1}{\sqrt{-\xi_{c} \mathscr{V}_{c}^{\prime}\left(\xi_{c}\right)(t-1)}}\right) d t \\
\quad=\left(-r_{0}^{2}\right)^{3} \int_{1}^{0} \frac{t^{2}}{r_{0}^{2}-t r_{0}^{2}}\left(\frac{1}{\sqrt{-\mathscr{V}_{0}\left(-t r_{0}^{2}\right)}}-\frac{1}{\sqrt{-4 r_{0}^{2} F(0)(t-1)}}\right) d t+o_{c \rightarrow 0}(1) \\
\quad=\int_{-r_{0}^{2}}^{0} \frac{\xi^{2}}{r_{0}^{2}+\xi}\left(\frac{1}{\sqrt{-\mathscr{V}_{0}(\xi)}}-\frac{1}{\sqrt{4 F(0)\left(\xi+r_{0}^{2}\right)}}\right) d \xi+o_{c \rightarrow 0}(1)
\end{aligned}
$$

The passage to the limit $c \rightarrow 0$ being justified by the dominated convergence theorem since the absolute value of the integrand is $\leq K t$ for $0 \leq t \leq 1 / 2$ for small $c$ and for $1 / 2 \leq t \leq 1$, since $\xi_{c}>-r_{0}^{2}$, 
$r_{0}^{2}+t \xi_{c} \geq r_{0}^{2}(1-t)$ and hence is equal to

$$
\begin{aligned}
& =\left|\frac{t^{2}}{r_{0}^{2}+t \xi_{c}} \cdot \frac{\mathscr{V}_{c}\left(t \xi_{c}\right)-\xi_{c} \mathscr{V}_{c}^{\prime}\left(\xi_{c}\right)(t-1)}{\sqrt{-\mathscr{V}_{c}\left(t \xi_{c}\right)} \sqrt{-\xi_{c} \mathscr{V}_{c}^{\prime}\left(\xi_{c}\right)(t-1)}\left[\sqrt{-\mathscr{V}_{c}\left(t \xi_{c}\right)}+\sqrt{-\xi_{c} \mathscr{V}_{c}^{\prime}\left(\xi_{c}\right)(t-1)}\right]}\right| \\
& \leq K \frac{(1-t)^{2}}{(1-t) \sqrt{1-t} \sqrt{1-t} \sqrt{1-t}}=\frac{K}{\sqrt{1-t}} \in L^{1}((1 / 2,1)) .
\end{aligned}
$$

Furthermore, letting $\xi=\xi_{c}+\left(r_{0}^{2}+\xi_{c}\right) t^{2}, t \geq 0$, the first integral in (31) is equal to

$$
\begin{aligned}
\frac{1}{\sqrt{r_{0}^{2}+\xi_{c}}} \int_{0}^{\sqrt{-\xi_{c} /\left(r_{0}^{2}+\xi_{c}\right)}} \frac{\left(\xi_{c}+\left(r_{0}^{2}+\xi_{c}\right) t^{2}\right)^{2}}{1+t^{2}} \cdot \frac{2 d t}{\sqrt{-\mathscr{V}_{c}^{\prime}\left(\xi_{c}\right)}} \\
=\frac{2}{\sqrt{r_{0}^{2}+\xi_{c}} \sqrt{-V_{c}^{\prime}\left(\xi_{c}\right)}}\left\{r_{0}^{4}\left[\frac{\pi}{2}-\arctan \sqrt{\frac{r_{0}^{2}+\xi_{c}}{-\xi_{c}}}\right]-2 r_{0}^{2}\left(r_{0}^{2}+\xi_{c}\right) \sqrt{\frac{-\xi_{c}}{r_{0}^{2}+\xi_{c}}}\right. \\
\left.\quad+\left(r_{0}^{2}+\xi_{c}\right)^{2}\left[\sqrt{\frac{-\xi_{c}}{r_{0}^{2}+\xi_{c}}}+\frac{1}{3} \sqrt{\frac{-\xi_{c}}{r_{0}^{2}+\xi_{c}}}\right]\right\},
\end{aligned}
$$

by direct computation. Since $\xi_{c} \simeq-r_{0}^{2}$ is a simple zero of $\mathscr{V}_{c}(\xi)=c^{2} \xi^{2}-4\left(r_{0}^{2}+\xi\right) F\left(r_{0}^{2}+\xi\right)$, we have

$$
\xi_{c}=-r_{0}^{2}+\frac{c^{2} r_{0}^{4}}{4 F(0)}+\frac{c^{4} r_{0}^{6}}{4 F(0)}\left(\frac{r_{0}^{2} f(0)}{F(0)}-2\right)+o_{c \rightarrow 0}\left(c^{4}\right)=-r_{0}^{2}+\frac{c^{2} r_{0}^{4}}{4 F(0)}+0_{c \rightarrow 0}\left(c^{4}\right)
$$

thus

$$
-\mathscr{V}_{c}^{\prime}\left(\xi_{c}\right)=4 F(0)+\mathrm{O}_{c \rightarrow 0}\left(c^{2}\right) \quad \text { and } \quad \frac{2}{\sqrt{r_{0}^{2}+\xi_{c}} \sqrt{-\mathscr{V}_{c}^{\prime}\left(\xi_{c}\right)}}=\frac{2}{r_{0}^{2} c}+\sigma_{c \rightarrow 0}(c) .
$$

As a consequence, the first integral in (31) is equal to

$$
\frac{r_{0}^{2} \pi}{c}+\left\{-\frac{r_{0}^{3}}{\sqrt{F(0)}}-\frac{2 r_{0}^{3}}{\sqrt{F(0)}}+\frac{r_{0}^{3}}{3 \sqrt{F(0)}}\right\}+\sigma_{c \rightarrow 0}(c)=\frac{r_{0}^{2} \pi}{c}-\frac{8 r_{0}^{3}}{3 \sqrt{F(0)}}+\sigma_{c \rightarrow 0}(c) .
$$

Gathering these two relations, we obtain

$$
P\left(U_{c}\right)=r_{0}^{2} \pi+c\left\{-\frac{8 r_{0}^{3}}{3 \sqrt{F(0)}}+\int_{-r_{0}^{2}}^{0} \frac{\xi^{2}}{r_{0}^{2}+\xi}\left(\frac{1}{\sqrt{-V_{0}(\xi)}}-\frac{1}{\sqrt{4 F(0)\left(\xi+r_{0}^{2}\right)}}\right) d \xi\right\}+o_{c \rightarrow 0}(c),
$$

as wished.

6B. Proof of Theorem 23. Since we have a kink solution $U_{0}$ for $c=0$, this implies that $\mathscr{V}_{0}(\xi)=$ $-4\left(r_{0}^{2}+\xi\right) F\left(r_{0}^{2}+\xi\right)$ is negative in $\left(-r_{0}^{2}, 0\right)$ and that $-r_{0}^{2}$ is a simple zero of $\mathscr{V}_{0}$; that is, $F(0)>0$. Then, $F>0$ in $\left[0, r_{0}^{2}\right)$ and

$$
F(\varrho) \simeq\left(\mathfrak{c}_{s}^{2} /\left(4 r_{0}^{2}\right)\right)\left(\varrho-r_{0}^{2}\right)^{2}
$$

for $\varrho \rightarrow r_{0}^{2}$, and it follows that there exists $K_{0}>0$ such that

$$
F(\varrho) \geq \frac{1}{K_{0}}\left(\varrho-r_{0}^{2}\right)^{2} .
$$


We consider for $\mu \geq 0$ the quantity

$$
\mathscr{K}_{\min }(\mu) \equiv \inf \left\{\mathscr{K}(u), u \in \mathscr{L}, \inf _{\mathbb{R}}|u|=\mu\right\} .
$$

The study of $\mathscr{K}_{\min }(0)$ is easy.

Proposition 6.1. We have

$$
\mathscr{K}_{\min }(0)=E\left(U_{0}\right)
$$

More precisely, for any $U \in \mathscr{L}$,

$$
E(U) \geq 4 \int_{\inf _{\mathbb{R}}|U|}^{r_{0}} \sqrt{F\left(s^{2}\right)} d s \quad \text { and } \quad E\left(U_{0}\right)=4 \int_{0}^{r_{0}} \sqrt{F\left(s^{2}\right)} d s .
$$

Finally, if $U \in \mathscr{L}, \inf _{\mathbb{R}}|U|=0$ and $\mathscr{K}(U)=E\left(U_{0}\right)$, then there exist $y \in \mathbb{R}$ and $\theta \in \mathbb{R}$ such that $U=\mathrm{e}^{i \theta} U_{0}(\cdot-y)$.

Proof. Taking $U_{0}$ as a comparison map, we see that $\mathscr{K}_{\min }(0) \leq E\left(U_{0}\right)$. Moreover, if $U \in \mathscr{Z}$ and $\inf _{\mathbb{R}}|U|=\mu \geq 0$, we may assume, up to a translation, that $\mu=|U|(0)$. Then, defining

$$
G(r) \equiv 2 \int_{r_{0}}^{r} \sqrt{F\left(s^{2}\right)} d s
$$

we have the inequalities

$$
\begin{aligned}
\int_{0}^{+\infty}\left|\partial_{x} U\right|^{2}+F\left(|U|^{2}\right) d x & \geq \int_{0}^{+\infty}\left|\partial_{x}\right| U||^{2}+F\left(|U|^{2}\right) d x \geq 2 \int_{0}^{+\infty}\left|\sqrt{F\left(|U|^{2}\right.} \partial_{x}\right| U|| d x \\
& =\int_{0}^{+\infty}\left|\partial_{x}[G(|U|)]\right| d x \geq\left|\int_{0}^{+\infty} \partial_{x}[G(|U|)] d x\right| \\
& =|G(|U|(+\infty))-G(|U|(0))|=\left|G\left(r_{0}\right)-G(\mu)\right|=2 \int_{\mu}^{r_{0}} \sqrt{F\left(s^{2}\right)} d s
\end{aligned}
$$

Arguing similarly in $(-\infty, 0)$, we get

$$
E(U) \geq 4 \int_{\mu}^{r_{0}} \sqrt{F\left(s^{2}\right)} d s .
$$

For the kink $U_{0}$, which is real-valued, we have the first integral $\left|\partial_{x} U_{0}\right|^{2}=F\left(U_{0}^{2}\right)$; hence, using the change of variables $s=U_{0}(x)$,

$$
E\left(U_{0}\right)=4 \int_{0}^{+\infty} F\left(U_{0}^{2}\right) d x=4 \int_{0}^{r_{0}} \sqrt{F\left(s^{2}\right)} d s .
$$

If $\mu=0$, we have then $E(U) \geq E\left(U_{0}\right)$; hence $\mathscr{K}(U) \geq E(U) \geq E\left(U_{0}\right)$ as wished.

Assume finally that $U \in \mathscr{E}$ satisfies $\inf _{\mathbb{R}}|U|=0$ and $\mathscr{K}(U)=E\left(U_{0}\right)$. Then $\mu=0$ and all the above inequalities are equalities. In particular, we must have $\left|\partial_{x} U\right|=\left|\partial_{x}\right| U||$ and equality in $\left|\partial_{x}\right| U||^{2}+F\left(|U|^{2}\right) \geq$ $2\left|\sqrt{F\left(|U|^{2}\right)} \partial_{x}\right| U||$, which means that $\left|\partial_{x}\right| U||=\sqrt{F\left(|U|^{2}\right)}$. Combining this ODE with the condition $|U|(0)=0$, we see that $|U|=\left|U_{0}\right|$, since $\left|U_{0}\right|$ solves $\partial_{x} U_{0}=\sqrt{F\left(U_{0}^{2}\right)}$. Finally, the fact that 
$\left|\partial_{x} U\right|=\left|\partial_{x}\right| U||$ implies that the phase is constant in $(-\infty, 0)$ and in $(0,+\infty)$ : there exist two constants $\theta_{ \pm} \in \mathbb{R}$ satisfying $U(x)=\mathrm{e}^{i \theta_{ \pm}}\left|U_{0}\right|(x)$ for $\pm x \geq 0$. Therefore, $\mathfrak{P}(U)=r_{0}^{2}\left(\theta_{+}-\theta_{-}\right) \bmod 2 \pi r_{0}^{2}$, and then

$$
E\left(U_{0}\right)=\mathscr{K}(u)=E\left(U_{0}\right)+2 M r_{0}^{4} \sin ^{2} \frac{\theta_{+}-\theta_{-}-\pi}{2}
$$

implies $\theta_{+}-\theta_{-}=\pi \bmod 2 \pi$; that is, $U=\mathrm{e}^{i \theta_{+}} U_{0}$ in $\mathbb{R}$, which is the desired result.

We recall the expansion $P\left(U_{s}\right)=r_{0}^{2} \pi+s \dot{P}_{0}+o(s)$ as $s \rightarrow 0$, where $\dot{P}_{0} \equiv\left(d P\left(U_{s}\right) / d s\right)_{\mid s=0}$. From the Hamilton group relation $d E\left(U_{s}\right) / d s=s d P\left(U_{s}\right) / d s$, we also infer by integration $E\left(U_{s}\right)=$ $E\left(U_{0}\right)+\frac{1}{2} s^{2} \dot{P}_{0}+o\left(s^{2}\right)$. As a first step, we define the small parameter $\mu_{*}>0$. The key point is to prove the following result.

Proposition 6.2. There exist some constant $K>0$ and a small $\mu_{*}>0$ such that, for any $0<\mu \leq \mu_{*}$,

$$
\mathscr{K}_{\text {min }}(\mu)=\inf \left\{\mathscr{K}(U), U \in \mathscr{L}, \inf _{\mathbb{R}}|u|=\mu\right\} \geq E\left(U_{0}\right)+\frac{\mu^{2}}{K} .
$$

Proof. Notice first that, for $c>0$ small, there exists $U_{c}$ traveling wave of speed $c$, and that $\inf _{\mathbb{R}}\left|U_{c}\right|=$ $\sqrt{r_{0}^{2}+\xi_{c}}$ with $\xi_{c}$ a smooth function in $c$ such that $\xi_{c}=-r_{0}^{2}+c^{2} r_{0}^{4} /(4 F(0))+\mathcal{O}\left(c^{4}\right)$; hence $\inf _{\mathbb{R}}\left|U_{c}\right|=$ $c r_{0}^{2} /(2 \sqrt{F(0)})+\mathcal{O}\left(c^{2}\right)$ and is smooth. Therefore, there exists, for $0 \leq \mu \leq \mu_{*}$ small, a unique $\sigma_{\mu}$, with $\sigma_{\mu}=2 \mu \sqrt{F(0)} / r_{0}^{2}+\mathcal{O}\left(\mu^{2}\right)$, such that $\mu=\inf _{\mathbb{R}}\left|U_{\sigma_{\mu}}\right|$. In particular, taking $U_{\sigma_{\mu}}$ as a comparison map in $K_{\min }(\mu)$, we have

$$
\begin{aligned}
\mathscr{K}_{\min }(\mu) & \leq \mathscr{K}\left(U_{\sigma_{\mu}}\right)=E\left(U_{\sigma_{\mu}}\right)+2 M r_{0}^{4} \sin ^{2} \frac{P\left(U_{\sigma_{\mu}}\right)-r_{0}^{2} \pi}{2 r_{0}^{2}} \\
& =E\left(U_{0}\right)+\frac{\sigma_{\mu}^{2}}{2} \dot{P}_{0}+o\left(\sigma_{\mu}^{2}\right)+2 M r_{0}^{4} \sin ^{2} \frac{\sigma_{\mu} \dot{P}_{0}+o\left(\sigma_{\mu}\right)}{2 r_{0}^{2}} \\
& =E\left(U_{0}\right)+\frac{\sigma_{\mu}^{2}}{2}\left(\dot{P}_{0}+M \dot{P}_{0}^{2}\right)+o\left(\sigma_{\mu}^{2}\right) .
\end{aligned}
$$

In particular, it follows that, for some positive constant $K$ and for $\mu_{*}$ small enough,

$$
\mathscr{K}_{\min }(\mu) \leq E\left(U_{0}\right)+K \mu^{2} \leq \frac{11}{10} E\left(U_{0}\right) .
$$

Consider now $c$ small, a bounded open interval $\left(x_{-}, x_{+}\right)$and $\eta$ a solution to the Newton equation

$$
2 \partial_{x}^{2} \eta+\mathscr{V}_{c}^{\prime}(\eta)=0
$$

in $\left(x_{-}, x_{+}\right)$, with $\partial_{x} \eta\left(x_{+}\right) \leq 0 \leq \partial_{x} \eta\left(x_{-}\right), \eta\left(x_{+}\right) \leq-r_{0}^{2}+\mu_{*}^{2}$ and $\eta\left(x_{-}\right) \leq-r_{0}^{2}+\mu_{*}^{2}$. As $c \rightarrow 0, \mathscr{V}_{c}$ converges to $\mathscr{V}_{0}$ in $\mathscr{C}^{1}\left(\left[-r_{0}^{2}, 0\right]\right)$. Moreover, $\mathscr{V}_{0}$ is negative in $\left(-r_{0}^{2}, 0\right)$ and has a simple zero at $-r_{0}^{2}$. Therefore, if $c$ and $\mu_{*}>0$ are sufficiently small, we must have $\int_{x_{-}}^{x_{+}} F\left(r_{0}^{2}+\eta\right) d x \geq \frac{1}{2} \int_{\mathbb{R}} F\left(U_{0}^{2}\right) d x$. Consequently, if $v=A \mathrm{e}^{i \varphi}$ solves $\left(\mathrm{TW}_{c}\right)$ on a bounded interval $\left(x_{-}, x_{+}\right)$, satisfies $2 \partial_{x} \varphi=c \eta /\left(r_{0}^{2}+\eta\right)$ $\left(\eta \equiv A^{2}-r_{0}^{2}\right)$ and if $|v|$ is $\leq \mu_{*}$ at $x_{+}$and at $x_{-}$, with $\partial_{x}|v|\left(x_{+}\right) \leq 0 \leq \partial_{x}|v|\left(x_{-}\right)$, then

$$
\int_{x_{-}}^{x_{+}}\left|\partial_{x} v\right|^{2}+F\left(|v|^{2}\right) d x \geq \frac{1}{2} E\left(U_{0}\right)
$$


Here, we use that the Newton equation on the modulus $|V|$ actually holds true in $\left(x_{-}, x_{+}\right)$. Since $F>0$ in $\left[0, r_{0}^{2}\right)$ and $F(\varrho) \simeq r_{0}^{2}\left(\varrho-r_{0}^{2}\right)^{2}$ when $\varrho \rightarrow r_{0}^{2}$, there exist $K>0$ and $\kappa>0$ such that $F(\varrho) \geq\left(\varrho-r_{0}^{2}\right)^{2} / K$ for $0 \leq \varrho \leq r_{0}^{2}(1+\kappa)^{2}$. Hence, if $\inf _{\mathbb{R}}|v| \geq \mu>0$, then

$$
|P(v)| \leq \frac{K}{\mu} E(v)
$$

Moreover, arguing as in the proof of Proposition 6.1, we show that there exists $\varkappa>0$ such that, if $U \in \mathscr{L}$ and $|U|$ takes values $\leq \mu_{*}$ and $\geq r_{0}(1+\kappa)$, then

$$
E(U) \geq E\left(U_{0}\right)(1+\varkappa) .
$$

In particular, since $\mathscr{K}_{\min }(\mu) \leq E\left(U_{0}\right)+\mathcal{O}\left(\mu^{2}\right)$, we may choose $\mu_{*}$ sufficiently small so that, if $U \in \mathscr{L}$ and $\mathscr{K}(U) \leq \mathscr{K}_{\min }(\mu)+\mu_{*}$, then $|U| \leq r_{0}(1+\kappa)$. This means that, for the mappings we are considering, $F(\varrho) \geq\left(\varrho-r_{0}^{2}\right)^{2} / K$.

Step 1: Construction of a suitable minimizing sequence. There exists a sequence $\left(V_{n}\right)_{n \geq 0}$ in $\mathscr{E}$ such that $\inf _{\mathbb{R}}\left|V_{n}\right|=\mu=\left|V_{n}\right|(0), V_{n}=A_{n} \mathrm{e}^{i \phi_{n}}, P\left(V_{n}\right) \in\left[0, \pi r_{0}^{2}\right]$,

$$
2 A_{n}^{2} \partial_{x} \phi_{n}=c_{n}\left(A_{n}^{2}-r_{0}^{2}\right), \quad c_{n} \equiv M r_{0}^{2} \sin \frac{r_{0}^{2} \pi-P\left(V_{n}\right)}{2 r_{0}^{2}} \geq 0 \quad \text { and } \quad \lim _{n \rightarrow+\infty} \mathscr{K}\left(V_{n}\right)=\mathcal{K}_{\min }(\mu) \text {. }
$$

Since $\mu>0$, the maps $V$ we consider may be lifted to $V=A \mathrm{e}^{i \phi}$. Therefore (with $u=\partial_{x} \phi$ ),

$$
\begin{array}{r}
\mathscr{K}_{\min }(\mu)=\inf \left\{\int_{\mathbb{R}}\left(\partial_{x} A\right)^{2}+F\left(A^{2}\right) d x+\inf \left\{\int_{\mathbb{R}} A^{2} u^{2} d x+2 M r_{0}^{4} \sin ^{2} \frac{\int_{\mathbb{R}}\left(A^{2}-r_{0}^{2}\right) u d x-r_{0}^{2} \pi}{2 r_{0}^{2}},\right.\right. \\
\left.\left.u \in L^{2}(\mathbb{R}, \mathbb{R})\right\}, A \in r_{0}+H^{1}(\mathbb{R}, \mathbb{R}), \inf _{\mathbb{R}} A=\mu\right\} .
\end{array}
$$

The infimum in $u$ may be written

$$
\inf _{p \in \mathbb{R}} \inf \left\{\int_{\mathbb{R}} A^{2} u^{2} d x+2 M r_{0}^{4} \sin ^{2} \frac{p-r_{0}^{2} \pi}{2 r_{0}^{2}}, u \in L^{2}(\mathbb{R}, \mathbb{R}) \text { s.t. } \int_{\mathbb{R}}\left(A^{2}-r_{0}^{2}\right) u d x=p\right\} .
$$

For each $p \in \mathbb{R}$, we minimize in $u$ a quadratic functional on an affine hyperplane, with minimizer given by

$$
u_{p}=p\left(\int_{\mathbb{R}} \frac{\left(A^{2}-r_{0}^{2}\right)^{2}}{A^{2}} d x\right)^{-1} \frac{A^{2}-r_{0}^{2}}{A^{2}}
$$

As a consequence, the infimum in $u$ in (35) is

$$
\inf _{p \in \mathbb{R}}\left[\int_{\mathbb{R}} A^{2} u_{p}^{2} d x+2 M r_{0}^{4} v \sin ^{2} \frac{p-r_{0}^{2} \pi}{2 r_{0}^{2}}\right]=\inf _{p \in \mathbb{R}}\left[p^{2}\left(\int_{\mathbb{R}} \frac{\left(A^{2}-r_{0}^{2}\right)^{2}}{A^{2}} d x\right)^{-1}+2 M r_{0}^{4} \sin ^{2} \frac{p-\pi r_{0}^{2}}{2 r_{0}^{2}}\right] .
$$

It is clear that this last infimum is achieved only for $p$ inside $\left[-\pi r_{0}^{2},+\pi r_{0}^{2}\right]$. Indeed, the second term is $2 \pi r_{0}^{2}$-periodic, and, if $p>\pi r_{0}^{2}$, then $p-2 \pi r_{0}^{2}$ is a better competitor. Moreover, the function $p \mapsto \sin ^{2}\left(\left(p-\pi r_{0}^{2}\right) /\left(2 r_{0}^{2}\right)\right)$ is continuous and even; hence we may consider some $p \in\left[0, \pi r_{0}^{2}\right]$ (depending 
on $A$ ), which is a minimizer for this last infimum. The corresponding $u_{p}$ is then a minimizer for the infimum in $u$ in (35). Writing that

$$
\begin{aligned}
\frac{d}{d p}\left[p^{2}\left(\int_{\mathbb{R}} \frac{\left(A^{2}-r_{0}^{2}\right)^{2}}{A^{2}} d x\right)^{-1}+2 M r_{0}^{4} \sin ^{2} \frac{p-\pi r_{0}^{2}}{2 r_{0}^{2}}\right] & =2 p\left(\int_{\mathbb{R}} \frac{\left(A^{2}-r_{0}^{2}\right)^{2}}{A^{2}} d x\right)^{-1}+2 M r_{0}^{2} \sin \frac{p-\pi r_{0}^{2}}{2 r_{0}^{2}}=0
\end{aligned}
$$

we deduce the relations

$$
2 A^{2} u=c\left(A^{2}-r_{0}^{2}\right), \quad c \equiv M r_{0}^{2} \sin \frac{p-\pi r_{0}^{2}}{2 r_{0}^{2}} .
$$

We conclude by considering a minimizing sequence $\left(A_{n}\right)$ in (35), and translating in space so that $\inf _{\mathbb{R}} A_{n}=\mu=\left|A_{n}\right|(0)$.

Since $F \geq 0$ in $\mathbb{R}_{+}$, we have

$$
\int_{\mathbb{R}}\left|\partial_{x} V_{n}\right|^{2} d x \leq \mathscr{K}\left(V_{n}\right) \leq \frac{12}{10} E\left(U_{0}\right)
$$

for $n$ large. Therefore, by the compact Sobolev embedding $H^{1}([-R,+R]) \hookrightarrow L^{\infty}([-R,+R])$, we may assume, up to a possible subsequence, that there exists $V \in H_{\text {loc }}^{1}(\mathbb{R})$ such that, for any $R>0, V_{n} \rightarrow V$ in $H^{1}([-R,+R])$ and $V_{n} \rightarrow V$ uniformly on $[-R,+R]$. Moreover, by lower semicontinuity and Fatou's lemma, $E(V) \leq \underline{\lim }_{n \rightarrow+\infty} E\left(V_{n}\right)$. Since $\left|V_{n}\right| \geq \mu>0$ in $\mathbb{R}$, we have $|V| \geq \mu>0$ in $\mathbb{R}$ and thus a lifting $V=A \mathrm{e}^{i \phi}$. Furthermore, $\inf _{\mathbb{R}} A_{n}=\mu=\left|V_{n}\right|(0)$; hence $\inf _{\mathbb{R}} A=\mu=|V|(0)$. We also know that $P\left(V_{n}\right) \in\left[0, r_{0}^{2} \pi\right]$ for all $n$; hence we may assume, up to another subsequence, that $P\left(V_{n}\right)$ converges to some $P_{\infty} \in\left[0, r_{0}^{2} \pi\right]$. We also set

$$
c \equiv \lim _{n \rightarrow+\infty} c_{n}=M r_{0}^{2} \sin \frac{P_{\infty}-\pi r_{0}^{2}}{2 r_{0}^{2}} .
$$

In view of Step 1 , and the convergence $A_{n} \rightarrow A$ uniformly on any compact interval $[-R,+R]$, it follows that

$$
2 A^{2} \partial_{x} \phi=c\left(A^{2}-r_{0}^{2}\right) \quad \text { and } \quad \partial_{x} \phi_{n} \rightarrow \partial_{x} \phi \quad \text { in } L_{\mathrm{loc}}^{\infty}(\mathbb{R})
$$

Note that

$$
\int_{\mathbb{R}}\left|\partial_{x} V\right|^{2}+\frac{1}{K}\left(|V|^{2}-r_{0}^{2}\right)^{2} d x \leq E(V)<+\infty ;
$$

hence $|V| \rightarrow r_{0}$ at $\pm \infty$. In particular, there exist $-\infty<R_{-} \leq 0 \leq R_{+}<+\infty$ such that $|V|>\mu$ in $\left(-\infty, R_{-}\right)$and in $\left(R_{+},+\infty\right)$ and $|V|\left(R_{ \pm}\right)=\mu$.

Step 2. There exist $-\infty<z_{-} \leq 0 \leq z_{+}<+\infty$ such that

$$
A(x)=A_{c}\left(x-R_{+}+z_{+}\right) \quad \text { for } x \geq R_{+} \quad \text { and } \quad A(x)=A_{c}\left(x-R_{-}+z_{-}\right) \quad \text { for } x \leq R_{-} .
$$

We work for $x \geq R_{+}$, the other case being similar. We consider $\chi \in \mathscr{C}_{c}^{1}\left(\left(R_{+},+\infty\right), \mathbb{C}\right), t \in \mathbb{R}$ small such that $V_{n}^{t} \equiv v_{n}+t \chi$ satisfies $\left|V_{n}^{t}\right|>\mu$ in $\left(R_{+},+\infty\right)$. This is possible since $\inf _{\operatorname{Supp}(\chi)}\left|V_{n}\right|>\mu$. Then, 
$\left|V_{n}^{t}\right| \geq \mu$ in $\mathbb{R}$ and $\left|V_{n}^{t}\right|(0)=\mu$; hence $V_{n}^{t}$ is then a comparison map for $\mathscr{K}_{\text {min }}(\mu)$, and, in view of the equality $P\left(V_{n}^{t}\right)=P\left(V_{n}\right)+2 t \int_{R_{+}}^{+\infty}\left\langle i \partial_{x} V_{n} \mid \chi\right\rangle d x+O\left(t^{2}\right)$, it follows that

$$
\begin{aligned}
\mathscr{K}_{\min }(\mu) \leq & \mathcal{K}\left(V_{n}^{t}\right) \\
= & \mathcal{K}_{\min }(\mu)+o_{n \rightarrow+\infty}(1)+2 t \int_{R_{+}}^{+\infty}\left\langle\partial_{x} V_{n}, \partial_{x} \chi\right\rangle d x+t^{2} \int_{R_{+}}^{+\infty}\left|\partial_{x} \chi\right|^{2} d x \\
& -2 t \int_{R_{+}}^{+\infty} f\left(\left|V_{n}\right|^{2}\right)\left\langle V_{n}, \chi\right\rangle d x+M t \sin \frac{P\left(V_{n}\right)-\pi r_{0}^{2}}{r_{0}^{2}} \int_{R_{+}}^{+\infty}\left\langle i \partial_{x} V_{n}, \chi\right\rangle d x+\bigcirc_{t \rightarrow 0}\left(t^{2}\right) .
\end{aligned}
$$

Letting $n \rightarrow+\infty$ and using the weak and strong convergences for $V_{n}$, we infer

$$
\begin{aligned}
0 \leq 2 t \int_{R_{+}}^{+\infty}\left\langle\partial_{x} V, \partial_{x} \chi\right\rangle d x-2 t \int_{R_{+}}^{+\infty} f\left(|V|^{2}\right)\langle V, \chi\rangle d x & -M t \sin \frac{r_{0}^{2} \pi-P_{\infty}}{2 r_{0}^{2}} \int_{R_{+}}^{+\infty}\left\langle i \partial_{x} V, \chi\right\rangle d x+O_{t \rightarrow 0}\left(t^{2}\right)
\end{aligned}
$$

Dividing by $t \neq 0$ and letting $t \rightarrow 0^{+}$and then $t \rightarrow 0^{-}$, we deduce that $V$ solves $\left(\mathrm{TW}_{c}\right)$ in $\left(R_{+},+\infty\right)$ and $V$ has finite energy. Moreover, $|V|\left(R_{+}\right)=\mu$ is small; thus $V=\mathrm{e}^{i \theta_{+}} U_{c}\left(\cdot-R_{+}+z_{+}\right)$in $\left(R_{+},+\infty\right)$ for some constants $z_{+}$and $\theta_{+}$, and the speed $c$ is such that $\inf _{\mathbb{R}} A_{c}=\sqrt{r_{0}^{2}+\xi_{c}} \leq \mu$; hence $c \leq \sigma(\mu) \leq K \mu$. Since $|V|$ has finite energy in $\mathbb{R}$ and solves $\left(\mathrm{TW}_{c}\right)$ in $\left(R_{+},+\infty\right), V$ is $\mathscr{C}^{1}$ in $\left[R_{+},+\infty\right)$. Moreover, $|V|$ reaches a minimum at $x=R_{+}$; thus we must have $\partial_{x}^{+}|V|\left(R_{+}\right) \geq 0$, which imposes $z_{+} \geq 0$. Note that, $A_{c}$ being even, it is possible to translate $V$ so that $R \equiv R_{+}=-R_{-}$and $z \equiv z_{+}=-z_{-}$. Observe that $\mu=A_{c}(z) \geq A_{0}(z)$; hence $z \leq K \mu$. This yields

$$
\int_{|x| \geq R}\left|\partial_{x} V\right|^{2}+F\left(|V|^{2}\right) d x=\int_{|x| \geq z}\left|\partial_{x} U_{c}\right|^{2}+F\left(\left|U_{c}\right|^{2}\right) d x \geq E\left(U_{0}\right)-K \mu .
$$

In particular, we deduce from (32)

$$
2 R F\left(\mu^{2}\right) \leq \int_{|x| \leq R}\left|\partial_{x} V\right|^{2}+F\left(|V|^{2}\right) d x \leq K \mu
$$

hence $R \leq K \mu$ for $\mu$ small $(F(0)>0)$.

Step 3. We prove that $A=\mu$ in $\left(R_{-}, R_{+}\right)=(-R,+R)$.

Indeed, if it is not the case, there exists a bounded interval $\left(x_{-}, x_{+}\right)$such that $A=|V|>\mu$ in $\left(x_{-}, x_{+}\right)$ and $|V|\left(x_{ \pm}\right)=\mu$, with $\partial_{x}|V|\left(x_{+}\right) \leq 0 \leq \partial_{x}|V|\left(x_{-}\right)$. Therefore, we can make perturbations of the amplitude $A_{n}$ localized in $\left(x_{-}, x_{+}\right)$. Hence, arguing as in Step 2, we see that, then, $V$ solves $\left(\mathrm{TW}_{c}\right)$ in $\left(x_{-}, x_{+}\right)$, with $2 A^{2} \partial_{x} \phi=c\left(A^{2}-r_{0}^{2}\right)$ and $|V|\left(x_{ \pm}\right)=\mu, \partial_{x}|V|\left(x_{+}\right) \leq 0 \leq \partial_{x}|V|\left(x_{-}\right)$. We then are in position to apply (33), yielding

$$
\int_{x_{-}}^{x_{+}}\left|\partial_{x} V\right|^{2}+F\left(|V|^{2}\right) d x \geq \frac{1}{2} E\left(U_{0}\right)
$$


but the combination with (37) provides

$$
\begin{aligned}
\frac{11}{10} E\left(U_{0}\right) \geq \mathcal{K}_{\min }(\mu) & \geq \int_{x_{-}}^{x_{+}}\left|\partial_{x} V\right|^{2}+F\left(|V|^{2}\right) d x+\int_{|x| \geq R}\left|\partial_{x} V\right|^{2}+F\left(|V|^{2}\right) d x \\
& \geq \frac{1}{2} E\left(U_{0}\right)+E\left(U_{0}\right)-K \mu_{*}=\frac{3}{2} E\left(U_{0}\right)-K \mu_{*},
\end{aligned}
$$

which is not possible if $\mu_{*}$ is sufficiently small.

Step 4. We have $R=0$ or $\left(z=0\right.$ and $\left.c=\sigma_{\mu}\right)$.

Indeed, assume $R>0$, and consider $\zeta \in \mathscr{C}_{c}^{1}((0,+\infty), \mathbb{R}), \zeta \geq 0, t \geq 0$ and $V_{n}^{t} \equiv\left(A_{n}+t \zeta\right) \mathrm{e}^{i \phi_{n}}$, so that $\left|V_{n}^{t}\right|=A_{n}+t \zeta \geq \mu$ in $\mathbb{R}$. Since $R>0$, we actually have $\inf _{\mathbb{R}}\left|V_{n}^{t}\right|=\mu$ and $V_{n}^{t}$ is a comparison map for $\mathcal{K}_{\min }(\mu)$. Arguing as before, we thus have

$$
\begin{aligned}
\mathscr{K}_{\min }(\mu) \leq \mathscr{K}\left(V_{n}^{t}\right)= & \mathcal{K}_{\min }(\mu)+o_{n \rightarrow+\infty}(1)+2 t \int_{0}^{+\infty} \partial_{x} A_{n} \partial_{x} \zeta d x+t^{2} \int_{0}^{+\infty}\left(\partial_{x} \zeta\right)^{2} d x \\
& +2 t \int_{0}^{+\infty} A_{n} \zeta\left(\partial_{x} \phi_{n}\right)^{2} d x+t^{2} \int_{0}^{+\infty} \zeta^{2}\left(\partial_{x} \phi_{n}\right)^{2} d x-2 t \int_{0}^{+\infty} f\left(A_{n}^{2}\right) A_{n} \zeta d x \\
& +M r_{0}^{2} t \sin \frac{P\left(V_{n}\right)-r_{0}^{2} \pi}{r_{0}^{2}} \int_{0}^{R} 2 A_{n} \zeta \partial_{x} \phi_{n} d x+\sigma_{t \rightarrow 0}\left(t^{2}\right)
\end{aligned}
$$

By (36), we may pass to the limit as $n \rightarrow+\infty$ in all the terms and deduce

$0 \leq 2 t \int_{0}^{+\infty} \partial_{x} A \partial_{x} \zeta d x+2 t \int_{0}^{+\infty} A \zeta\left(\partial_{x} \phi\right)^{2} d x-2 t \int_{0}^{+\infty} f\left(A^{2}\right) A \zeta d x-2 c t \int_{0}^{+\infty} A \zeta \partial_{x} \phi d x+\widehat{O}_{t \rightarrow 0}\left(t^{2}\right)$.

At this stage, we see the relevance of taking a minimizing sequence as chosen in Step 1, since it allows us to pass to the limit in the nonlinear terms involving $\partial_{x} \phi_{n}$. As a consequence, using (36),

$$
-\partial_{x}^{2} A-A f\left(A^{2}\right)+\frac{c^{2}}{4} \frac{\left(A^{2}-r_{0}^{2}\right)^{2}}{A^{3}} \geq 0
$$

in the distributional sense in $(0,+\infty)$. The term $-A f\left(A^{2}\right)+\frac{1}{4} c^{2}\left(A^{2}-r_{0}^{2}\right)^{2} / A^{3}$ is continuous in $\mathbb{R}$. However, since $A(x)=\mu$ for $0 \leq x \leq R$ and $A(x)=A_{c}(x-R+z)$ for $x \geq R$, we infer $-\partial_{x}^{2} A=$ $-\partial_{x} A_{c}(z) \delta_{x=R}$ plus a piecewise continuous function in the distributional sense in $(0,+\infty)$. Since $\partial_{x} A_{c}(z) \geq 0$ (recall that $z \geq 0$ ), this forces $\partial_{x} A_{c}(z)=0$; that is, $z=0$. Consequently, $\mu=|V|(R)=$ $A(R)=A_{c}(z)=A_{c}(0)$ and then $c=\sigma_{\mu}$.

In the next step, we take into account the loss in the weak convergence $V_{n} \rightarrow V$.

Step 5. There exists $K>0$ such that

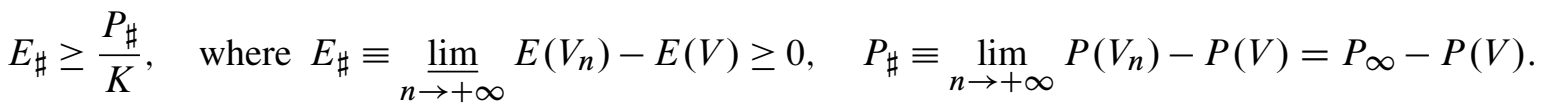

Let $\epsilon>0$ be fixed but small, and pick some $X>0$ large so that

$$
\left.\left|E(V)-\int_{|x| \leq X}\right| \partial_{x} V\right|^{2}+F\left(|V|^{2}\right) d x|\leq \epsilon, \quad| P(V)-\int_{|x| \leq X}\left(A^{2}-r_{0}^{2}\right) u d x \mid \leq \epsilon .
$$


We claim that there exists some small $\bar{\mu}>0$, independent of $\epsilon$, such that $\left|V_{n}\right| \geq \bar{\mu}$ for $|x| \geq X$ and $n$ large. Indeed, otherwise, we may argue as in Step 3 and show, as in the beginning of the proof there, that $\int_{|x| \geq X}\left|\partial_{x} V_{n}\right|^{2}+F\left(\left|V_{n}\right|^{2}\right) d x \geq \frac{1}{2} E\left(U_{0}\right)$. This is not possible since

$$
\frac{12}{10} E\left(U_{0}\right) \geq \lim _{n \rightarrow+\infty} E\left(V_{n}\right) \geq \frac{1}{2} E\left(U_{0}\right)+\int_{|x| \leq X}\left|\partial_{x} V\right|^{2}+F\left(|V|^{2}\right) d x \geq \frac{1}{2} E\left(U_{0}\right)+E(V)-\epsilon,
$$

and $E(V)$ is close to $E\left(U_{0}\right)$ as $\mu \rightarrow 0$. Therefore, as for (34),

$$
\left|\int_{|x| \geq X}\left(A_{n}^{2}-r_{0}^{2}\right) u_{n} d x\right| \leq \frac{K}{\bar{\mu}} \int_{|x| \geq X}\left|\partial_{x} V_{n}\right|^{2}+F\left(\left|V_{n}\right|^{2}\right) d x .
$$

Consequently,

$$
\begin{aligned}
& E\left(V_{n}\right)-E(V) \\
& \geq \int_{|x| \leq X}\left|\partial_{x} V_{n}\right|^{2}+F\left(\left|V_{n}\right|^{2}\right) d x-\int_{|x| \leq X}\left|\partial_{x} V\right|^{2}+F\left(|V|^{2}\right) d x+\int_{|x| \geq X}\left|\partial_{x} V_{n}\right|^{2}+F\left(\left|V_{n}\right|^{2}\right) d x-\epsilon \\
& \geq \int_{|x| \leq X}\left|\partial_{x} V_{n}\right|^{2}+F\left(\left|V_{n}\right|^{2}\right) d x-\int_{|x| \leq X}\left|\partial_{x} V\right|^{2}+F\left(|V|^{2}\right) d x+\frac{\bar{\mu}}{K}\left|\int_{|x| \geq X}\left(A_{n}^{2}-r_{0}^{2}\right) u_{n} d x\right|-\epsilon .
\end{aligned}
$$

Passing to the liminf and using the weak convergence in $[-X,+X]$, we infer

$$
\varliminf_{n \rightarrow+\infty} E\left(V_{n}\right)-E(V) \geq \frac{\bar{\mu}}{K} \varliminf_{n \rightarrow+\infty}\left|P\left(V_{n}\right)-\int_{|x| \leq X}\left(A_{n}^{2}-r_{0}^{2}\right) u_{n} d x\right|-\epsilon .
$$

However, (36) implies

so that

$$
\int_{|x| \leq X}\left(A_{n}^{2}-r_{0}^{2}\right) u_{n} d x \rightarrow \int_{|x| \leq X}\left(A^{2}-r_{0}^{2}\right) u d x
$$

$$
E_{\#} \geq \frac{\bar{\mu}}{K}\left|P_{\infty}-\int_{|x| \leq X}\left(A^{2}-r_{0}^{2}\right) u d x\right|-\epsilon \geq \frac{\bar{\mu}}{K}\left|P_{\infty}-P(V)\right|-\left(1+\frac{\bar{\mu}}{K}\right) \epsilon=\frac{\bar{\mu}}{K}\left|P_{\sharp}\right|-\left(1+\frac{\bar{\mu}}{K}\right) \epsilon .
$$

Letting $\epsilon \rightarrow 0$, the conclusion follows.

Step 6. There exists $K>0$ such that, if $R>0$, then

$$
\mathscr{K}_{\min }(\mu) \geq E\left(U_{0}\right)+\frac{\mu^{2}}{K} .
$$

We recall the expansion $P\left(U_{s}\right)=r_{0}^{2} \pi+s \dot{P}_{0}+o(s)$ as $s \rightarrow 0$, where $\dot{P}_{0} \equiv\left(d P\left(U_{s}\right) / d s\right)_{\mid s=0}$. From the Hamilton group relation $d E\left(U_{s}\right) / d s=s d P\left(U_{s}\right) / d s$, we also infer by integration $E\left(U_{s}\right)=$ $E\left(U_{0}\right)+\frac{1}{2} s^{2} \dot{P}_{0}+o\left(s^{2}\right)$. On the other hand, by definition of $c_{n}$,

$$
\begin{aligned}
2 M r_{0}^{4} \sin ^{2} \frac{P\left(V_{n}\right)-r_{0}^{2} \pi}{2 r_{0}^{2}} & =M r_{0}^{4}\left[1-\cos \frac{P\left(V_{n}\right)-r_{0}^{2} \pi}{r_{0}^{2}}\right] \\
& =M r_{0}^{4}\left[1-\sqrt{1-\sin ^{2} \frac{P\left(V_{n}\right)-r_{0}^{2} \pi}{r_{0}^{2}}}\right]=M r_{0}^{4}\left[1-\sqrt{1-\frac{c_{n}^{2}}{M^{2}}}\right]
\end{aligned}
$$


for $n$ large. Here, we have used that $M c_{n}=\sin \left(\left(r_{0}^{2} \pi-P\left(V_{n}\right)\right) / r_{0}^{2}\right) \rightarrow M c \in\left[0, K \mu_{*}\right]$ (cf. Step 2); thus $\cos \left(\left(r_{0}^{2} \pi-P\left(V_{n}\right)\right) / r_{0}^{2}\right) \geq 0$, for, otherwise, we would have, by Proposition 6.1,

$$
\begin{aligned}
\mathscr{K}\left(V_{n}\right)=E\left(V_{n}\right)+2 M r_{0}^{4} \sin ^{2} \frac{P\left(V_{n}\right)-r_{0}^{2} \pi}{2 r_{0}^{2}} & \geq E\left(U_{0}\right)-K \mu+M r_{0}^{4}\left[1+\sqrt{1-\frac{c_{n}^{2}}{M^{2}}}\right] \\
& \geq E\left(U_{0}\right)-K \mu_{*}+2 M r_{0}^{4}+\mathcal{O}\left(\mu_{*}^{2}\right),
\end{aligned}
$$

but this contradicts (32) if $\mu_{*}$ is sufficiently small.

We assume $R>0$, so that, by Step $4, z=0$ and $c=\sigma_{\mu}$. We recall $\sigma_{\mu}=2 \mu \sqrt{F(0)} / r_{0}^{2}+\mathcal{O}\left(\mu^{2}\right) \sim$ $2 \mu \sqrt{F(0)} / r_{0}^{2}$. By definition of $E_{\sharp}$, one has

$$
\begin{aligned}
E_{\sharp}+E(V)+M r_{0}^{4}\left[1-\sqrt{1-\frac{c^{2}}{M^{2}}}\right] & \leq \lim _{n \rightarrow+\infty} E\left(V_{n}\right)+\lim _{n \rightarrow+\infty} 2 M r_{0}^{4} \sin ^{2} \frac{P\left(V_{n}\right)-r_{0}^{2} \pi}{2 r_{0}^{2}} \\
& =\varliminf_{n \rightarrow+\infty} \mathscr{\operatorname { l i m } _ { n }} \mathscr{T}\left(V_{n}\right)=\mathcal{K}_{\min }(\mu)
\end{aligned}
$$

since $\left(V_{n}\right)$ is minimizing for $\mathscr{K}_{\min }(\mu)$. Moreover, from the expression of $V$, we have (for $R>0$ )

$$
E(V)=E\left(U_{\sigma_{\mu}}\right)+2 R\left[\frac{\sigma_{\mu}^{2}\left(r_{0}^{2}-\mu^{2}\right)^{2}}{4 \mu^{2}}+F\left(\mu^{2}\right)\right] \quad \text { and } \quad P(V)=P\left(U_{\sigma_{\mu}}\right)+R \sigma_{\mu} \frac{\left(r_{0}^{2}-\mu^{2}\right)^{2}}{\mu^{2}} .
$$

Furthermore, we have $P_{\#}=P_{\infty}-P(V)$ and $c=M r_{0}^{2} \sin \left(\left(r_{0}^{2} \pi-P_{\infty}\right) / r_{0}^{2}\right)$ with $P_{\infty} \in\left[0, r_{0}^{2} \pi\right]$ and $\cos \left(\left(r_{0}^{2} \pi-P_{\infty}\right) / r_{0}^{2}\right) \geq 0$; thus

$$
P_{\sharp}=P_{\infty}-P(V)=r_{0}^{2} \pi-r_{0}^{2} \arcsin \left(\frac{c}{M r_{0}^{2}}\right)-P\left(U_{\sigma_{\mu}}\right)-R \sigma_{\mu} \frac{\left(r_{0}^{2}-\mu^{2}\right)^{2}}{\mu^{2}} .
$$

Combining this with the expansions of $E\left(U_{\sigma}\right)$ and $P\left(U_{\sigma}\right)$ gives

$$
\begin{aligned}
\mathscr{K}_{\min }(\mu) \geq E\left(U_{0}\right)+E_{\sharp}+\frac{\sigma_{\mu}^{2}}{2} \dot{P}_{0}+o\left(\sigma_{\mu}^{2}\right)+M\left[1-\sqrt{1-\frac{\sigma_{\mu}^{2}}{M^{2}}}\right]+2 R\left[\frac{\sigma_{\mu}^{2}\left(r_{0}^{2}-\mu^{2}\right)^{2}}{4 \mu^{2}}+F\left(\mu^{2}\right)\right] \\
\geq E\left(U_{0}\right)+\frac{\left|P_{\sharp}\right|}{K}+\frac{\sigma_{\mu}^{2}}{2}\left[\dot{P}_{0}+\frac{1}{M}\right]+o\left(\mu^{2}\right)+4 R F(0) \\
\geq E\left(U_{0}\right)+\frac{1}{K}\left|r_{0}^{2} \arcsin \left(\sigma_{\mu} /\left(M r_{0}^{2}\right)\right)+\sigma_{\mu} \dot{P}_{0}+R \sigma \frac{\left(r_{0}^{2}-\mu^{2}\right)^{2}}{\mu^{2}}+o\left(\sigma_{\mu}\right)\right|+\frac{\sigma_{\mu}^{2}}{2}\left[\dot{P}_{0}+\frac{1}{M}\right] \\
+o\left(\mu^{2}\right)+4 R F(0) \\
\geq E\left(U_{0}\right)+\frac{1}{K}\left|\frac{\sigma_{\mu}}{M}+\sigma_{\mu} \dot{P}_{0}+R \sigma_{\mu} \frac{\left(r_{0}^{2}-\mu^{2}\right)^{2}}{\mu^{2}}+o\left(\sigma_{\mu}\right)\right|+\frac{\sigma_{\mu}^{2}}{2}\left[\dot{P}_{0}+\frac{1}{M}\right]+o\left(\mu^{2}\right)+4 R F(0) \\
\geq E\left(U_{0}\right)+\frac{\sigma_{\mu}}{K}\left|\dot{P}_{0}+\frac{1}{M}+R \frac{\left(r_{0}^{2}-\mu^{2}\right)^{2}}{\mu^{2}}\right|+\frac{\sigma_{\mu}^{2}}{2}\left[\dot{P}_{0}+\frac{1}{M}\right]+4 R F(0)+o\left(\mu^{2}\right) .
\end{aligned}
$$

The right-hand side is a continuous piecewise affine function of $R$ (the " $O$ " does not depend on $R$ ). Since $\sigma_{\mu}\left(r_{0}^{2}-\mu^{2}\right)^{2} /\left(K \mu^{2}\right) \simeq 1 / \mu \gg 4 F(0)$ and $\dot{P}_{0}+1 / M<0$ (since $M>-\dot{P}_{0}^{-1}$ by hypothesis), it 
follows that the right-hand side is a function of $R$ which is decreasing in $\left[0, R_{0}(\mu)\right]$ and increasing in $\left[R_{0}(\mu),+\infty\right)$, with

$$
R_{0}(\mu) \equiv-\left(\dot{P}_{0}+\frac{1}{M}\right) \frac{\mu^{2}}{\left(r_{0}^{2}-\mu^{2}\right)^{2}} \sim-\left(\dot{P}_{0}+\frac{1}{M}\right) \frac{\mu^{2}}{r_{0}^{4}}>0
$$

Therefore, using once again that $\sigma_{\mu}^{2} \sim 4 \mu^{2} F(0) / r_{0}^{4}$,

$$
\begin{aligned}
\mathscr{K}_{\min }(\mu) & \geq E\left(U_{0}\right)+\frac{\sigma_{\mu}^{2}}{2}\left[\dot{P}_{0}+\frac{1}{M}\right]+4 R_{0}(\mu) F(0)+o\left(\mu^{2}\right) \\
& =E\left(U_{0}\right)+\left[\dot{P}_{0}+\frac{1}{M}\right] \frac{2 \mu^{2} F(0)}{r_{0}^{4}}-\left[\dot{P}_{0}+\frac{1}{M}\right] \frac{4 \mu^{2} F(0)}{r_{0}^{4}}+o\left(\mu^{2}\right) \\
& =E\left(U_{0}\right)-\mu^{2} \frac{2 F(0)}{r_{0}^{4}}\left[\dot{P}_{0}+\frac{1}{M}\right]+o\left(\mu^{2}\right) .
\end{aligned}
$$

In view of our hypothesis $\dot{P}_{0}+1 / M<0$, we infer that

$$
\mathcal{K}_{\min }(\mu) \geq E\left(U_{0}\right)+\frac{\mu^{2}}{K}
$$

for $\mu_{*}$ sufficiently small and some positive constant $K$, as wished. If the assumption $\dot{P}_{0}+1 / M<0$ is not satisfied, but, if $\dot{P}_{0}+1 / M>0$ for instance, then the function of $R$ above is increasing in $[0,+\infty)$, with minimum value achieved at $R=0$ and equal to

$$
E\left(U_{0}\right)+\frac{\sigma_{\mu}^{2}}{2}\left[\dot{P}_{0}+\frac{1}{M}\right]+o\left(\mu^{2}\right)=E\left(U_{0}\right)+\frac{2 \mu^{2} F(0)}{r_{0}^{4}}\left[\dot{P}_{0}+\frac{1}{M}\right]+o\left(\mu^{2}\right) \geq E\left(U_{0}\right)+\frac{\mu}{K}
$$

We then would have concluded a stronger estimate, which is actually in contradiction with (32); hence we are necessarily in the case $R>0$. The assumption $\dot{P}_{0}+1 / M<0$ is however crucial for the last step.

Step 7. We assume $\dot{P}_{0}+1 / M<0$. Then, for $\mu_{*}$ sufficiently small, the case $R=0$ does not occur.

We argue in a similar way, but, since $R=0$, the expressions for $E(V)$ and $P(V)$ are given by

$$
E(V)=E\left(U_{c}\right)-4 \int_{0}^{z} F\left(\left|U_{c}\right|^{2}\right) d x \quad \text { and } \quad P(V)=P\left(U_{c}\right)-2 \int_{0}^{z} \frac{c}{2} \frac{\left(r_{0}^{2}-A_{c}^{2}\right)^{2}}{A_{c}^{2}} d x
$$

Here, we have used that $\left|\partial_{x} U_{c}\right|^{2}=F\left(\left|U_{c}\right|^{2}\right)$ since $U_{c}$ solves $\left(\mathrm{TW}_{c}\right)$. Combining this here again with the expansions of $E\left(U_{c}\right)$ and $P\left(U_{c}\right)$ gives, using that $0 \leq c \leq K \mu$,

$$
\begin{aligned}
\mathscr{K}_{\min }(\mu) & \geq E\left(U_{0}\right)+E_{\sharp}+\frac{c^{2}}{2} \dot{P}_{0}+o\left(c^{2}\right)+M\left[1-\sqrt{1-\frac{c^{2}}{M^{2}}}\right]-4 \int_{0}^{z} F\left(\left|U_{c}\right|^{2}\right) d x \\
& \geq E\left(U_{0}\right)+\frac{\left|P_{\sharp}\right|}{K}+\frac{c^{2}}{2}\left[\dot{P}_{0}+\frac{1}{M}\right]-4 z F(0)+o\left(\mu^{2}\right) \\
& \geq E\left(U_{0}\right)+\frac{1}{K}\left|\arcsin (c / M)+c \dot{P}_{0}-c \int_{0}^{z} \frac{\left(r_{0}^{2}-A_{c}^{2}\right)^{2}}{A_{c}^{2}} d x+o(c)\right|+\frac{c^{2}}{2}\left[\dot{P}_{0}+\frac{1}{M}\right]+o\left(\mu^{2}\right)-4 z F(0) .
\end{aligned}
$$


Following the lines of the proof of Lemma 20, we have

$$
c \int_{0}^{z} \frac{\left(r_{0}^{2}-A_{c}^{2}\right)^{2}}{A_{c}^{2}} d x=2 \arctan \sqrt{\frac{\mu^{2}}{r_{0}^{2}+\xi_{c}}}+O\left(\mu^{2}\right) .
$$

Indeed, noticing that $A_{c}=\mathcal{O}(\mu)$ in $[0, z]$ with $z \leq K \mu$, we write, expanding the square,

$$
\int_{0}^{z} \frac{\left(r_{0}^{2}-A_{c}^{2}\right)^{2}}{A_{c}^{2}} d x=\int_{0}^{z} \frac{r_{0}^{4}}{A_{c}^{2}}-2+A_{c}^{2} d x=\int_{0}^{z} \frac{r_{0}^{4}}{A_{c}^{2}} d x+\mathcal{O}(\mu) .
$$

Then, using the change of variable $\xi=\eta_{c}(x)$,

$$
\begin{aligned}
\int_{0}^{z} \frac{\left(r_{0}^{2}-A_{c}^{2}\right)^{2}}{A_{c}^{2}} d x= & \int_{\xi_{c}}^{\mu^{2}-r_{0}^{2}} \frac{r_{0}^{4}}{\left(r_{0}^{2}+\xi\right) \sqrt{-\mathscr{V}_{c}(\xi)}} d \xi+O(\mu) \\
= & \int_{\xi_{c}}^{\mu^{2}-r_{0}^{2}} \frac{r_{0}^{4}}{\left(r_{0}^{2}+\xi\right) \sqrt{-\mathscr{V}_{c}^{\prime}\left(\xi_{c}\right)\left(\xi-\xi_{c}\right)}} d \xi \\
& \quad+\int_{\xi_{c}}^{\mu^{2}-r_{0}^{2}} \frac{r_{0}^{4}}{\left(r_{0}^{2}+\xi\right)}\left(\frac{1}{\sqrt{-\mathscr{V}_{c}(\xi)}}-\frac{1}{\sqrt{-\mathscr{V}_{c}^{\prime}\left(\xi_{c}\right)\left(\xi-\xi_{c}\right)}}\right) d \xi+O(\mu) \\
= & \frac{2}{c} \arctan \sqrt{\frac{\mu^{2}}{r_{0}^{2}+\xi_{c}}-1}+\mathcal{O}(\mu)
\end{aligned}
$$

by computations similar to those for the proof of Lemma 20. This proves (38). Therefore,

$\mathcal{K}_{\min }(\mu)$

$\geq E\left(U_{0}\right)+\frac{1}{K}\left|c\left[\dot{P}_{0}+\frac{1}{M}\right]-2 \arctan \sqrt{\frac{\mu^{2}}{r_{0}^{2}+\xi_{c}}-1}+o(c)\right|+\frac{c^{2}}{2}\left[\dot{P}_{0}+\frac{1}{M}\right]+o\left(\mu^{2}\right)-4 z F(0)$.

By (32), the left-hand side is $\leq E\left(U_{0}\right)+K \mu^{2}$. Since $\dot{P}_{0}+1 / M<0, c \leq K \mu, z \leq K \mu$ and $F(0)>0$, this implies

$$
\left|c\left[\dot{P}_{0}+\frac{1}{M}\right]-2 \arctan \sqrt{\frac{\mu^{2}}{r_{0}^{2}+\xi_{c}}}+o(c)\right| \leq K \mu
$$

thus

$$
\arctan \sqrt{\frac{\mu^{2}}{r_{0}^{2}+\xi_{c}}-1} \leq K \mu,
$$

and, finally, for $\mu_{*}$ small enough,

$$
0 \leq \frac{\mu^{2}}{r_{0}^{2}+\xi_{c}}-1 \leq K \mu^{2} .
$$

Combining this with the equality $r_{0}^{2}+\xi_{c}=c^{2} r_{0}^{4} /(4 F(0))+\mathcal{O}\left(c^{4}\right)$ seen during the proof of Lemma 20, we infer

$$
c=\frac{2 \sqrt{F(0)}}{r_{0}^{2}} \mu+\mathcal{O}\left(\mu^{2}\right) .
$$


In particular, going back to (39) and since, for $0 \leq x \leq z$,

$$
r_{0}^{2}+\xi_{c}=A_{c}^{2}(0) \leq A_{c}^{2}(x) \leq A_{c}^{2}(z)=\mu^{2},
$$

this implies

$$
\frac{z r_{0}^{4}}{\mu^{2}} \leq \int_{0}^{z} \frac{r_{0}^{4}}{A_{c}^{2}} \leq \frac{2}{c} \arctan \sqrt{\frac{\mu^{2}}{r_{0}^{2}+\xi_{c}}-1}+\mathcal{O}(\mu) \leq \frac{K \mu}{c}+K \mu \leq K,
$$

which provides (since $c \approx \mu$ )

$$
z \leq K \mu^{2} .
$$

Inserting this into (40) and keeping in mind that the left-hand side is $\leq E\left(U_{0}\right)+K \mu^{2}$, we deduce

$$
c\left[\dot{P}_{0}+\frac{1}{M}\right]-2 \arctan \sqrt{\frac{\mu^{2}}{r_{0}^{2}+\xi_{c}}-1}=o(\mu) .
$$

However, since $\arctan \sqrt{\mu^{2} /\left(r_{0}^{2}+\xi_{c}\right)-1} \geq 0$, this gives

$$
o(\mu) \leq c\left[\dot{P}_{0}+\frac{1}{M}\right] \sim \frac{2 \mu \sqrt{F(0)}}{r_{0}^{2}}\left[\dot{P}_{0}+\frac{1}{M}\right],
$$

yielding a contradiction for small $\mu$ since we have $\dot{P}_{0}+1 / M<0$ by assumption. Therefore, the case $R=0$ does not occur for sufficiently small $\mu_{*}$. If we had $\dot{P}_{0}+1 / M>0$, we would not have been able to show that $\mathscr{K}_{\min }(\mu)$ gives a control on $\mu$.

The proof of Proposition 6.2 is complete.

Proof of Theorem 23. Let $U \in \mathscr{V}_{\mu_{*}}$. If $\mu \equiv \inf _{\mathbb{R}}|U|>0$, then Proposition 6.2 gives $\mathscr{K}(U) \geq$ $E\left(U_{0}\right)+\mu^{2} / K>E\left(U_{0}\right)=\mathscr{K}\left(U_{0}\right)$. If $\inf _{\mathbb{R}}|U|=0$, we deduce from Proposition 6.1 that $\mathscr{K}(U) \geq$ $E\left(U_{0}\right)+2 M r_{0}^{4} \sin ^{2}\left(\left(\mathfrak{P}(U)-\pi r_{0}^{2}\right) r_{0}^{2}\right)$. Hence $\mathscr{K}(U)>E\left(U_{0}\right)$ except if $\mathscr{K}(U)=E\left(U_{0}\right)$. From the study of the equality case in Proposition 6.1, it follows that $U \in\left\{\mathrm{e}^{i \theta} U_{0}(\cdot-y), y \in \mathbb{R}, \theta \in \mathbb{R}\right\}$, as claimed.

6C. Proof of Theorem 24. As a first step, we shall need a quantified version of Proposition 6.1.

Proposition 6.3. There exist $\epsilon_{0}>0$ and $K>0$, depending only on $f$, such that, for any $U \in \mathscr{L}$ verifying

$$
\mathscr{K}(U)-E\left(U_{0}\right) \leq \epsilon_{0} \quad \text { and } \quad \inf _{\mathbb{R}}|U| \leq \epsilon_{0},
$$

we have

$$
\inf _{\substack{y \in \mathbb{R} \\ \theta \in \mathbb{R}}} d_{\mathscr{E}}\left(U, \mathrm{e}^{i \theta} U_{0}(\cdot-y)\right) \leq K\left(\mathscr{K}(U)-E\left(U_{0}\right)+\inf _{\mathbb{R}}|U|\right)^{1 / 4} .
$$

Proof. First, we translate the problem in space so that $\mu \equiv \inf _{\mathbb{R}}|U|=|U|(0)$ and shall choose the phase factor later. We follow the lines of the proof of Proposition 6.1 and actually get (writing $U=A \mathrm{e}^{i \phi}$ locally 
in $\{|U|>0\})$

$$
\begin{aligned}
& \int_{0}^{+\infty}\left|\partial_{x} U\right|^{2}+F\left(|U|^{2}\right) d x \\
& \quad=\int_{0}^{+\infty} \mathbf{1}_{|U|>0} A^{2}\left(\partial_{x} \phi\right)^{2} d x+\int_{0}^{+\infty}\left|\partial_{x}\right| U||^{2}+F\left(|U|^{2}\right) d x \\
& \quad=\int_{0}^{+\infty} \mathbf{1}_{|U|>0} A^{2}\left(\partial_{x} \phi\right)^{2} d x+\int_{0}^{+\infty}\left[\sqrt{F\left(|U|^{2}\right)}-\left|\partial_{x}\right| U||\right]^{2} d x+2 \int_{0}^{+\infty}\left|\sqrt{F\left(|U|^{2}\right)} \partial_{x}\right| U|| d x \\
& \quad \geq \int_{0}^{+\infty} \mathbf{1}_{|U|>0} A^{2}\left(\partial_{x} \phi\right)^{2} d x+\int_{0}^{+\infty}\left[\sqrt{F\left(|U|^{2}\right)}-\left|\partial_{x}\right| U||\right]^{2} d x+2 \int_{\mu}^{r_{0}} \sqrt{F\left(s^{2}\right)} d s .
\end{aligned}
$$

Arguing similarly in $(-\infty, 0)$, we get

$$
E(U) \geq E\left(U_{0}\right)+\int_{\mathbb{R}} \mathbf{1}_{|U|>0} A^{2}\left(\partial_{x} \phi\right)^{2} d x+\int_{\mathbb{R}}\left[\sqrt{F\left(|U|^{2}\right)}-\left|\partial_{x}\right| U||\right]^{2} d x-4 \int_{0}^{\mu} \sqrt{F\left(s^{2}\right)} d s .
$$

The gradient of the phase is controlled using (41). We shall now estimate the modulus part. Let us define $A \equiv|U|$ and

$$
h \equiv \partial_{x} A-\sqrt{F\left(A^{2}\right)},
$$

for which we have, by (41),

$$
\|h\|_{L^{2}(\mathbb{R})}^{2} \leq E(U)-E\left(U_{0}\right)+4 \int_{0}^{\mu} \sqrt{F\left(s^{2}\right)} d s \leq E(U)-E\left(U_{0}\right)+K \mu .
$$

Recall that $U_{0}$ satisfies $\left(\partial_{x} U_{0}\right)^{2}=F\left(U_{0}^{2}\right)$ in $\mathbb{R}$; hence $\partial_{x} U_{0}=\sqrt{F\left(U_{0}^{2}\right)}$ in $\mathbb{R}_{+}$. Setting $\Theta \equiv A-\left|U_{0}\right|$, we infer

$$
\partial_{x} \Theta=\sqrt{F\left(A^{2}\right)}-\sqrt{F\left(U_{0}^{2}\right)}+h \text { in } \mathbb{R}_{+} .
$$

We set, for $x \geq 0$,

$$
G(x, \theta) \equiv \sqrt{F\left(\left(U_{0}(x)+\theta\right)^{2}\right)}-\sqrt{F\left(U_{0}^{2}(x)\right)}+\frac{U_{0}(x) f\left(U_{0}^{2}(x)\right) \theta}{\sqrt{F\left(U_{0}^{2}(x)\right)}} .
$$

Since $U_{0}$ satisfies $\partial_{x}^{2} U_{0}+U_{0}(x) f\left(U_{0}^{2}(x)\right)=0$ and $\partial_{x} U_{0}=\sqrt{F\left(U_{0}^{2}(x)\right)}$ in $\mathbb{R}_{+}$, it follows that

$$
G(x, \theta)=\sqrt{F\left(\left(U_{0}(x)+\theta\right)^{2}\right)}-\sqrt{F\left(U_{0}^{2}(x)\right)}-\frac{\partial_{x}^{2} U_{0}(x)}{\partial_{x} U_{0}(x)} \theta .
$$

Moreover, by the Taylor expansion, we infer the existence of $K>0$ and $\theta_{0}>0$ such that, for $|\theta| \leq \theta_{0}$, $x \in \mathbb{R}_{+}$,

$$
|G(x, \theta)| \leq K \theta^{2} .
$$

The estimate is clearly uniform in view of the exponential decay of $\partial_{x} U_{0}$ at infinity. Therefore,

$$
\partial_{x} \Theta=\frac{\partial_{x}^{2} U_{0}(x)}{\partial_{x} U_{0}(x)} \Theta+G(x, \Theta)+h(x) .
$$


We view this ODE as a linear ODE with source term $G(x, \Theta(x))+h(x)$. Since $\partial_{x} U_{0}$ solves the homogeneous equation, we infer, from Duhamel's formula and the fact that $\Theta(0)=A(0)-U_{0}(0)=$ $|U(0)|=\mu$, that, for $x \geq 0$,

$$
\Theta(x)=\mu+\partial_{x} U_{0}(x) \int_{0}^{x} \frac{G(z, \Theta(z))+h(z)}{\partial_{x} U_{0}(z)} d z .
$$

We shall prove that this equation implies that, if $\mu$ and $\|h\|_{L^{2}\left(\mathbb{R}_{+}\right)}$are sufficiently small, then

$$
\|\Theta\|_{L^{2}\left(\mathbb{R}_{+}\right)} \leq K\left(\|h\|_{L^{2}\left(\mathbb{R}_{+}\right)}+\mu\right) .
$$

We assume $\mu<\theta_{0} / 2$. Note that, since $U_{0}$ is a kink, we have the decays given in Proposition 2. Hence, there exist two positive constants $K_{1}$ and $K_{2}$ such that

$$
\frac{\mathrm{e}^{-\mathfrak{c}_{s} x}}{K_{1}} \leq \partial_{x} U_{0}(x) \leq K_{2} \mathrm{e}^{-\mathfrak{c}_{s} x} \quad \text { for all } x \in \mathbb{R}_{+} .
$$

In particular, if $|\Theta(x)| \leq \theta_{0}$ in the interval $[0, R]$, then (44) implies, for $x \in[0, R]$,

$$
\begin{aligned}
|\Theta(x)| & \leq \mu+K_{1} K_{2} \mathrm{e}^{-\mathfrak{c}_{s} x} \int_{0}^{x} \mathrm{e}^{\mathfrak{c}_{s} z}\left[K\|\Theta\|_{L^{\infty}([0, R])}|\Theta(z)|+|h|(z)\right] d z \\
& \leq \mu+\frac{K K_{1} K_{2}}{\mathfrak{c}_{s}}\|\Theta\|_{L^{\infty}([0, R])}^{2}+\frac{K_{1} K_{2}}{\sqrt{2 \mathfrak{c}_{s}}}\|h\|_{L^{2}\left(\mathbb{R}_{+}\right)}
\end{aligned}
$$

by the Cauchy-Schwarz inequality. We thus choose $\|h\|_{L^{2}\left(\mathbb{R}_{+}\right)}+\mu$ sufficiently small so that

$$
4\left(\mu+\frac{K_{1} K_{2}}{\sqrt{2 \mathfrak{c}_{s}}}\|h\|_{L^{2}(\mathbb{R})}\right) \leq \tilde{\theta}_{0} \equiv \min \left\{\theta_{0}, \frac{\mathfrak{c}_{s}}{2 K K_{1} K_{2}}\right\} .
$$

Then, we consider the set $\mathscr{R}$ of all $R>0$ such that $|\Theta(x)| \leq \tilde{\theta}_{0}$ in the interval $[0, R]$. Since $\Theta \in H^{1}(\mathbb{R}, \mathbb{C})$ is continuous by the Sobolev embedding and $|\Theta(0)|=\mu<\tilde{\theta}_{0}, \mathscr{R} \neq \varnothing$ and is closed in $\mathbb{R}_{+}^{*}$. Moreover, the above estimate shows that, for $R \in \mathscr{R}$,

$$
\|\Theta\|_{L^{\infty}([0, R])} \leq \mu+\frac{K K_{1} K_{2}}{\mathfrak{c}_{s}}\|\Theta\|_{L^{\infty}([0, R])}^{2}+\frac{K_{1} K_{2}}{\sqrt{2 \mathfrak{c}_{s}}}\|h\|_{L^{2}(\mathbb{R}+)}
$$

which gives

$$
\|\Theta\|_{L^{\infty}([0, R])}\left(1-\frac{K K_{1} K_{2}}{\mathfrak{c}_{S}}\|\Theta\|_{L^{\infty}([0, R])}\right) \leq \mu+\frac{K_{1} K_{2}}{\sqrt{2 \mathfrak{c}_{S}}}\|h\|_{L^{2}\left(\mathbb{R}_{+}\right)},
$$

and then

$$
\|\Theta\|_{L^{\infty}([0, R])} \leq 2\left[\mu+\frac{K_{1} K_{2}}{\sqrt{2 \mathfrak{c}_{s}}}\|h\|_{L^{2}(\mathbb{R})}\right] \leq \frac{\tilde{\theta}_{0}}{2}<\tilde{\theta}_{0} .
$$

Consequently, $\mathscr{R}$ is open in $\mathbb{R}_{+}^{*}$. By connexity, $\mathscr{R}=\mathbb{R}_{+}^{*}$, proving (45). In what follows, we assume $\|h\|_{L^{2}\left(\mathbb{R}_{+}\right)}+\mu$ is sufficiently small so that $\|\Theta\|_{L^{\infty}} \leq \tilde{\theta}_{0}$; thus $|G(x, \Theta)| \leq K \Theta^{2}$. In particular,

$$
|\Theta(x)| \leq \mu+K_{1} K_{2} \int_{0}^{x} \mathrm{e}^{-\mathfrak{c}_{s}(x-z)}\left[K\|\Theta\|_{L^{\infty}([0, R])}|\Theta(z)|+|h|(z)\right] d z .
$$


For $R>0$ to be determined later, we then deduce from classical convolution estimates that

$$
\|\Theta\|_{L^{2}([0, R])} \leq \mu \sqrt{R}+K_{3}\|\Theta\|_{L^{\infty}\left(\mathbb{R}_{+}\right)}\|\Theta\|_{L^{2}([0, R])}+K_{3}\|h\|_{L^{2}\left(\mathbb{R}_{+}\right)} .
$$

Imposing that $\|h\|_{L^{2}\left(\mathbb{R}_{+}\right)}+\mu$ be smaller if necessary, we may assume that

$$
K_{3}\|\Theta\|_{L^{\infty}\left(\mathbb{R}_{+}\right)} \leq K_{3} K\left(\|h\|_{L^{2}\left(\mathbb{R}_{+}\right)}+\mu\right) \leq \frac{1}{2}
$$

so that we get

$$
\|\Theta\|_{L^{2}([0, R])} \leq K_{4}\left(\mu \sqrt{R}+\|h\|_{L^{2}\left(\mathbb{R}_{+}\right)}\right) .
$$

Reporting this into (43) provides

$$
\left\|\partial_{x} \Theta\right\|_{L^{2}([0, R])}^{2} \leq K_{5}\left(\mu^{2} R+\|h\|_{L^{2}\left(\mathbb{R}_{+}\right)}^{2}\right) .
$$

Arguing similarly in $[-R, 0]$ and using (42), we obtain an $H^{1}$ estimate for $\Theta$ in $[-R, R]$ :

$$
\|\Theta\|_{H^{1}([-R, R])}^{2} \leq K_{6}\left(E(U)-E\left(U_{0}\right)+\mu^{2} R+\mu\right)
$$

We now turn to the estimate in $\{|x| \geq R\}$. For that purpose, we write

$$
\begin{aligned}
\int_{|x| \geq R}\left(\partial_{x}|U|\right)^{2}+\frac{1}{K}\left(|U|^{2}-r_{0}^{2}\right)^{2} d x & =E(U)-E\left(U_{0}\right)+\int_{|x| \geq R}\left(\partial_{x} U_{0}\right)^{2}+F\left(U_{0}^{2}\right) d x \\
& -\int_{|x| \leq R}\left(\partial_{x}|U| \mid\right)^{2}+F\left(|U|^{2}\right) d x+\int_{|x| \leq R}\left(\partial_{x} U_{0}\right)^{2}+F\left(U_{0}^{2}\right) d x .
\end{aligned}
$$

Since $U_{0}$ decays exponentially (see Proposition 2), it follows that

$$
\int_{|x| \geq R}\left|\partial_{x} U_{0}\right|^{2}+F\left(U_{0}^{2}\right) d x \leq K \mathrm{e}^{-\mathfrak{c}_{S} R} .
$$

Furthermore, by integration by parts,

$$
\begin{aligned}
& -\int_{|x| \leq R}\left(\partial_{x}|U|\right)^{2}+F\left(|U|^{2}\right) d x+\int_{|x| \leq R}\left(\partial_{x} U_{0}\right)^{2}+F\left(U_{0}^{2}\right) d x \\
& \quad=-\int_{|x| \leq R} 2 \partial_{x} U_{0} \partial_{x} \Theta-2 U_{0} f\left(U_{0}^{2}\right) \Theta d x-\int_{|x| \leq R}\left(\partial_{x} \Theta\right)^{2}+F\left(\left[U_{0}+\Theta\right]^{2}\right)-F\left(U_{0}^{2}\right)-2 U_{0} F^{\prime}\left(U_{0}^{2}\right) \Theta d x \\
& \leq \int_{|x| \leq R} 2 \Theta\left[\partial_{x}^{2} U_{0}+U_{0} f\left(U_{0}^{2}\right)\right] d x-2 \Theta(+R) \partial_{x} U_{0}(+R)+2 \Theta(-R) \partial_{x} U_{0}(-R)+K\|\Theta\|_{H^{1}([-R,+R])}^{2} \\
& \leq K \mathrm{e}^{-\mathfrak{c}_{S} R}+K\left(E(U)-E\left(U_{0}\right)+\mu^{2} R+\mu\right) .
\end{aligned}
$$

For the second-to-last line, we have used that $\theta \mapsto F\left(\left[U_{0}+\theta\right]^{2}\right)-F\left(U_{0}^{2}\right)-2 U_{0} F^{\prime}\left(U_{0}^{2}\right) \theta$ is $O\left(\theta^{2}\right)$ as $\theta \rightarrow 0$ and, for the last line, that $U_{0}$ solves $\partial_{x}^{2} U_{0}+U_{0} f\left(U_{0}^{2}\right)=0$, the exponential decay of $\partial_{x} U_{0}$ and 
the uniform bound on $\Theta$. Reporting these estimates into (48) provides

$$
\begin{aligned}
\|\Theta\|_{H^{1}(\{|x| \geq R\})}^{2} & =\int_{|x| \geq R}\left(\partial_{x}|U|-\partial_{x}\left|U_{0}\right|\right)^{2}+\left(|U|-\left|U_{0}\right|\right)^{2} d x \\
& \leq 2 \int_{|x| \geq R}\left(\partial_{x}|U|\right)^{2}+\left(\partial_{x}\left|U_{0}\right|\right)^{2}+\left(|U|-r_{0}\right)^{2}+\left(\left|U_{0}\right|-r_{0}\right)^{2} d x \\
& \leq K\left[E(U)-E\left(U_{0}\right)+\mathrm{e}^{-\mathfrak{c}_{s} R}+\mu^{2} R+\mu\right] .
\end{aligned}
$$

Combining this with (47), we deduce that, for any $R>0$, we have

$$
\|\Theta\|_{H^{1}(\mathbb{R})}^{2} \leq K\left[E(U)-E\left(U_{0}\right)+\mathrm{e}^{-\mathfrak{c}_{S} R}+\mu^{2} R+\mu\right] .
$$

We then choose $R=\mu^{-1}$ if $\mu>0$ or $R \rightarrow+\infty$ if $\mu=0$, and get

$$
\|\Theta\|_{H^{1}(\mathbb{R})} \leq K \sqrt{E(U)-E\left(U_{0}\right)+\mu} .
$$

Notice that, if $f^{\prime}<0$ everywhere, then we may give a quick proof of the above estimate, since, using here again integration by parts and that $\partial_{x}^{2} U_{0}+U_{O} f\left(U_{O}^{2}\right)=0$, we may deduce that

$$
E(U)-E\left(U_{0}\right) \geq-4 \mu \partial_{x} U_{0}(0)+\int_{0}^{+\infty}\left(\partial_{x} \Theta\right)^{2} d x+\int_{\mathbb{R}} F\left(\left(U_{0}+\Theta\right)^{2}\right)-F\left(U_{0}^{2}\right)-2 U_{0} \Theta F^{\prime}\left(U_{0}^{2}\right) d x
$$

and, since $f^{\prime}<0, F\left(\left(U_{0}+\theta\right)^{2}\right)-F\left(U_{0}^{2}\right)-2 U_{0} \theta F^{\prime}\left(U_{0}^{2}\right) \geq \theta^{2} / K$ by the Taylor expansion, providing the desired $H^{1}$ bound on $\Theta$.

Observe now that

hence

$$
\mathscr{K}(U)-E\left(U_{0}\right) \geq E(U)-E\left(U_{0}\right) \geq \int_{\mathbb{R}} \mathbf{1}_{|U|>0}\left[A \partial_{x} \phi\right]^{2} d x
$$

$$
\begin{aligned}
\left\|\partial_{x} U-\partial_{x} U_{0}\right\|_{L^{2}(\mathbb{R})} & =\left\|\partial_{x}\left(\left|U_{0}\right|+\Theta\right) \mathrm{e}^{i \phi} \mathbf{1}_{|U|>0}+i \mathbf{1}_{|U|>0} A \partial_{x} \phi \mathrm{e}^{i \phi}-\partial_{x} U_{0}\right\|_{L^{2}(\mathbb{R})} \\
& \leq\left\|\mathrm{e}^{i \phi} \mathbf{1}_{|U|>0} \partial_{x}\left|U_{0}\right|-\partial_{x} U_{0}\right\|_{L^{2}(\mathbb{R})}+\left\|\mathbf{1}_{|U|>0} A \partial_{x} \phi\right\|_{L^{2}(\mathbb{R})}+\|\Theta\|_{L^{2}(\mathbb{R})} \\
& \leq\left\|\mathrm{e}^{i \phi} \mathbf{1}_{|U|>0} \partial_{x}\left|U_{0}\right|-\partial_{x} U_{0}\right\|_{L^{2}(\mathbb{R})}+K\left[\mathscr{K}(U)-E\left(U_{0}\right)+\mu\right]^{1 / 2} .
\end{aligned}
$$

We distinguish now the cases $\mu=0$ and $\mu>0$, and begin with the assumption $\mu>0$. Then, we have a global lifting $U=A \mathrm{e}^{i \phi}$ and

$$
\begin{aligned}
d_{\mathscr{L}}\left(U, U_{0}\right) & =\left\|\partial_{x} U-\partial_{x} U_{0}\right\|_{L^{2}(\mathbb{R})}+\left\||U|-\left|U_{0}\right|\right\|_{L^{2}(\mathbb{R})}+\left|U(0)-U_{0}(0)\right| \\
& =\left\|\partial_{x} U-\partial_{x} U_{0}\right\|_{L^{2}(\mathbb{R})}+\|\Theta\|_{L^{2}(\mathbb{R})}+\mu \\
& \leq\left\|e^{i \phi} \partial_{x}\left|U_{0}\right|-\partial_{x} U_{0}\right\|_{L^{2}(\mathbb{R})}+K\left[\mathcal{K}(U)-E\left(U_{0}\right)+\mu\right]^{1 / 2}
\end{aligned}
$$

Now, we notice that

$$
\begin{aligned}
\left\|\mathrm{e}^{i \phi} \partial_{x}\left|U_{0}\right|-\partial_{x} U_{0}\right\|_{L^{2}(\mathbb{R})}^{2} & =2 \int_{\mathbb{R}}\left[\left(\partial_{x} U_{0}\right)^{2}-\partial_{x} U_{0} \partial_{x}\left|U_{0}\right| \cos \phi\right] d x \\
& =2 \int_{0}^{+\infty}\left(\partial_{x} U_{0}\right)^{2}(1-\cos \phi) d x+2 \int_{-\infty}^{0}\left(\partial_{x} U_{0}\right)^{2}(1+\cos \phi) d x
\end{aligned}
$$


and that

$$
\mathscr{K}(U)-E\left(U_{0}\right) \geq 2 M r_{0}^{4} \sin ^{2} \frac{\mathfrak{P}(U)-r_{0}^{2} \pi}{2 r_{0}^{2}} \geq \frac{1}{K}\left(P(U)-r_{0}^{2} \pi \bmod 2 \pi r_{0}^{2}\right)^{2}
$$

We define $\delta=\left(\mathscr{K}(U)-E\left(U_{0}\right)+\mu\right)^{1 / 4}$. By the Cauchy-Schwarz inequality, we have

$$
\begin{aligned}
\left|\int_{|x| \geq \delta}\left(A^{2}-r_{0}^{2}\right) \partial_{x} \phi d x\right| & \leq \frac{K}{\inf _{|x| \geq \delta} A}\left(\int_{|x| \geq \delta}\left(A^{2}-r_{0}^{2}\right)^{2} d x\right)^{1 / 2}\left(\int_{|x| \geq \delta}\left(A \partial_{x} \phi\right)^{2} d x\right)^{1 / 2} \\
& \leq \frac{K}{\inf _{|x| \geq \delta} A}\left(E(U)-E\left(U_{0}\right)+\mu\right)^{1 / 2} .
\end{aligned}
$$

Inserting this into (51) gives

$$
\begin{aligned}
\left|\int_{|x| \leq \delta}\left(A^{2}-r_{0}^{2}\right) \partial_{x} \phi d x-r_{0}^{2} \pi \bmod 2 \pi r_{0}^{2}\right| & \leq K\left[\left(\mathscr{K}(U)-E\left(U_{0}\right)\right)^{1 / 2}+\frac{1}{\inf _{|x| \geq \delta} A}\left(E(U)-E\left(U_{0}\right)+\mu\right)^{1 / 2}\right] \\
& \leq \frac{K}{\inf _{|x| \geq \delta} A}\left(\mathscr{K}(U)-E\left(U_{0}\right)+\mu\right)^{1 / 2} .
\end{aligned}
$$

In addition, by the Cauchy-Schwarz inequality,

$$
\left|\int_{|x| \leq \delta} A^{2} \partial_{x} \phi d x\right| \leq \sqrt{2 \delta}\left(\sup _{|x| \leq \delta} A\right)\left(\mathscr{K}(U)-E\left(U_{0}\right)+\mu\right)^{1 / 2} .
$$

Consequently,

$$
\begin{aligned}
r_{0}^{2} \mid \phi( & +\delta)-\phi(-\delta)-\pi \bmod 2 \pi \mid \\
& \leq\left|\int_{|x| \leq \delta}\left(A^{2}-r_{0}^{2}\right) \partial_{x} \phi d x-r_{0}^{2} \pi \bmod 2 \pi r_{0}^{2}\right|+\sqrt{2 \delta}\left(\sup _{|x| \leq \delta} A\right)\left(\mathscr{K}(U)-E\left(U_{0}\right)+\mu\right)^{1 / 2} \\
& \leq\left[\frac{K}{\inf _{|x| \geq \delta} A}+\sqrt{2 \delta}\left(\sup _{|x| \leq \delta} A\right)\right]\left(\mathscr{K}(U)-E\left(U_{0}\right)+\mu\right)^{1 / 2}
\end{aligned}
$$

From our choice $\delta=\left(\mathscr{K}(U)-E\left(U_{0}\right)+\mu\right)^{1 / 4} \ll 1$ and since $\|\Theta\|_{L^{\infty}(\mathbb{R})} \leq K\left(\mathscr{K}(U)-E\left(U_{0}\right)+\mu\right)^{1 / 2}=$ $\mathcal{O}\left(\delta^{2}\right)$, we infer $\inf _{|x| \geq \delta} A \geq \inf _{|x| \geq \delta}\left|U_{0}\right|-\|\Theta\|_{L^{\infty}(\mathbb{R})} \geq \delta / K$. Similarly, we have $\sup _{|x| \leq \delta} A \leq$ $\sup _{|x| \leq \delta}\left|U_{0}\right|+\|\Theta\|_{L^{\infty}(\mathbb{R})} \leq K \delta$. Reporting this into (52) yields

$$
|\phi(+\delta)-\phi(-\delta)-\pi \bmod 2 \pi| \leq K \delta .
$$

We now freeze the gauge invariance by imposing $\phi(+\delta)=0$. Note that then $\phi(-\delta)=\pi+O(\delta)$. Furthermore, since $\phi(+\delta)=0$,

$$
\int_{|x| \geq \delta}\left(\partial_{x} \phi\right)^{2} d x \leq \frac{K}{\left(\inf _{|x| \geq \delta} A\right)^{2}} \int_{|x| \geq \delta} A^{2}\left(\partial_{x} \phi\right)^{2} d x \leq \frac{K}{\delta^{2}} \delta^{4}=K \delta^{2},
$$

which implies, for $x \geq \delta$,

$$
|1-\cos \phi(x)| \leq|1-\cos \phi(0)|+\left|\int_{\delta}^{x} \partial_{x} \phi \sin \phi\right| \leq K \delta \sqrt{x}
$$


and, similarly, since $\cos \phi(-\delta)=\cos (\pi+\mathscr{O}(\delta))=-1+\mathcal{O}\left(\delta^{2}\right)$, for $x \leq-\delta$,

$$
|1+\cos \phi(x)| \leq K \delta \sqrt{|x|} .
$$

We turn back to (50) and infer

$$
\begin{aligned}
\left\|e^{i \phi} \partial_{x}\left|U_{0}\right|-\partial_{x} U_{0}\right\|_{L^{2}(\mathbb{R})}^{2} & \leq K \delta+2 \int_{\delta}^{+\infty}\left(\partial_{x} U_{0}\right)^{2}(1-\cos \phi) d x+2 \int_{-\infty}^{-\delta}\left(\partial_{x} U_{0}\right)^{2}(1+\cos \phi) d x \\
& \leq K \delta+K \delta \int_{\mathbb{R}}\left(\partial_{x} U_{0}\right)^{2} \sqrt{|x|} d x=K \delta .
\end{aligned}
$$

Inserting these estimates in (49), it follows that

$$
d_{\mathscr{Q}}\left(U, U_{0}\right) \leq K \delta .
$$

We now turn to the case $\mu=0$. Without loss of generality, we may assume that $|U|>0$ in $(-\infty, 0)$ (since $|U| \rightarrow r_{0}>0$ at $\pm \infty$ ), and let $\ell \geq 0$ be such that $|U|(\ell)=0$ and $|U|>0$ in $(\ell,+\infty)$. We first estimate $\ell$ by writing that

$$
\left|U_{0}\right|(\ell)=|U|(\ell)+\Theta(\ell)=\Theta(\ell) \leq\|\Theta\|_{L^{\infty}(\mathbb{R})} \leq K\left(\mathscr{K}(U)-E\left(U_{0}\right)+\mu\right)^{1 / 2}=K \delta^{2} ;
$$

thus $\ell \leq K \delta^{2}$. Moreover, we have two local liftings $U=A \mathrm{e}^{i \phi_{+}}$in $[\ell,+\infty)$ and $U=A \mathrm{e}^{i \phi_{-}}$in $(-\infty, 0)$. Going back to (49), we then deduce

$$
\begin{aligned}
& d_{\mathscr{L}}\left(U, U_{0}\right) \\
& \quad \leq\left\|\mathrm{e}^{i \phi_{-}} \partial_{x}\left|U_{0}\right|-\partial_{x} U_{0}\right\|_{L^{2}(-\infty, 0)}+\left\|\mathrm{e}^{i \phi_{+}} \partial_{x}\left|U_{0}\right|-\partial_{x} U_{0}\right\|_{L^{2}(\ell,+\infty)}+K \delta+K\left[\mathscr{K}(U)-E\left(U_{0}\right)\right]^{1 / 2} .
\end{aligned}
$$

Arguing as for the case $\mu>0$, we obtain $|U|=A \geq \delta / K$ in $[\ell+\delta,+\infty)$ and in $(-\infty,-\delta)$. By definition of $\mathfrak{P}$, we have

$\mathfrak{P}(U)=\int_{-\delta}^{\ell+\delta}\left\langle i U \mid \partial_{x} U\right\rangle+\int_{\ell+\delta}^{+\infty}\left(A^{2}-r_{0}^{2}\right) \partial_{x} \phi_{+} d x+r_{0}^{2} \phi_{+}(\ell+\delta)+\int_{-\infty}^{-\delta}\left(A^{2}-r_{0}^{2}\right) \partial_{x} \phi_{-} d x-r_{0}^{2} \phi_{+}(-\delta)$ in $\mathbb{R} /\left(2 \pi r_{0}^{2} \mathbb{Z}\right)$; hence the same arguments as in the case $\mu>0$ provide

$$
\left|\phi_{+}(\ell+\delta)-\phi_{+}(-\delta)-\pi \bmod 2 \pi\right| \leq K \delta,
$$

since the integral $\int_{-\delta}^{\ell+\delta}\left\langle i U \mid \partial_{x} U\right\rangle$ is bounded by $K \sqrt{\delta}$ by the Cauchy-Schwarz inequality. Imposing $\phi_{+}(\ell+\delta)$ for the gauge invariance, we infer $1-\cos \left(\phi_{+}(\ell+\delta)\right)=0$ and $\phi_{+}(-\delta)=\pi+\mathcal{O}(\sqrt{\delta}) \bmod 2 \pi$; hence $1+\cos \left(\phi_{-}(-\delta)\right)=\mathscr{O}(\delta)$. Therefore, we conclude as before that

$$
d_{\mathscr{L}}\left(U, U_{0}\right) \leq K \delta,
$$

which finishes the proof of the proposition.

In order to prove Theorem 24, we use Proposition 6.2, which provides

$$
\mathscr{K}(U) \geq E\left(U_{0}\right)+\frac{1}{K}\left(\inf _{\mathbb{R}}|U|\right)^{2}
$$


thus

$$
\mu=\inf _{\mathbb{R}}|U| \leq K \sqrt{\mathscr{\mathscr { C }}(U)-E\left(U_{0}\right)} .
$$

Inserting this bound in Proposition 6.3 then gives

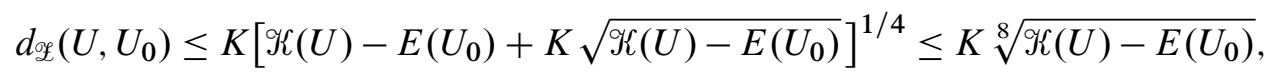

and the proof is complete.

\section{About the stability analysis for the sonic waves $\left(c=\mathfrak{c}_{s}\right)$}

We have left aside in our study the case of the sonic waves $\left(c=\mathfrak{c}_{s}\right)$, but would like to say a few words on the difficulties associated with this critical case.

We note that, if there exists a sonic nontrivial traveling wave, it does not vanish; hence we may use the hydrodynamical formulation (15) of (NLS) as in [Lin 2002]. The point is that the Sturm-Liouville operator (see [Lin 2002, Section 4])

$$
L \equiv-\frac{\partial}{\partial x}\left(\frac{1}{4\left(r_{0}^{2}-\eta\right)} \frac{\partial}{\partial x}\right)+q(x)
$$

with

$$
q(x) \equiv \frac{\left(\partial_{x} \eta\right)^{2}}{4\left(r_{0}^{2}-\eta\right)^{3}}-\frac{\partial}{\partial x}\left(\frac{\partial_{x} \eta}{4\left(r_{0}^{2}-\eta\right)^{2}}\right)-\frac{1}{2} f^{\prime}\left(r_{0}^{2}-\eta\right)-\frac{c^{2} r_{0}^{4}}{4\left(r_{0}^{2}-\eta\right)^{3}},
$$

has, by Weyl's theorem, essential spectrum $\sigma_{\text {ess }}(L)=[0,+\infty)$ when $c=\mathfrak{c}_{s}$. Indeed, we know from Proposition 2 that $\eta_{c_{s}}$ and its derivatives tend to zero at infinity; hence, as $x \rightarrow \pm \infty, q(x) \rightarrow$ $-\frac{1}{2} f^{\prime}\left(r_{0}^{2}\right)-c^{2} /\left(4 r_{0}^{2}\right)=0$ since $c^{2}=\mathfrak{c}_{s}^{2}=-2 r_{0}^{2} f^{\prime}\left(r_{0}^{2}\right)$. Therefore, there does not exist $\delta>0$ such that $\langle H p, p\rangle \geq \delta\|p\|^{2}$ for any $p$ orthogonal to the subspace spanned by the negative and the zero eigenvalue, and thus the Grillakis-Shatah-Strauss theory does not apply.

In the case $(d P / d c)_{\mid c=\mathfrak{c}_{s}}<0$, where it is natural to expect stability, a natural thing would be to try to work with the functional

$$
\mathscr{L}(\psi) \equiv E(\psi)-\mathfrak{c}_{s} P(\psi)+\frac{M}{2}\left(P(\psi)-P\left(U_{\mathfrak{c}_{s}}\right)\right)^{2}
$$

and to follow the lines of the proof of Theorem 23. Indeed, the spectral analysis shall not give positive definiteness of the Hessian due to presence of essential spectrum down to 0 . Therefore, we may study $\mathscr{L}$ at fixed $\mu=\inf _{\mathbb{R}}|\psi|$ close to $\inf _{\mathbb{R}}\left|U_{\mathfrak{c}_{s}}\right|$. When $0<c_{*}<\mathfrak{c}_{s}$ and $(d P / d c)_{\mid c=c_{*}} \neq 0$, the infimum of $\left|U_{c}\right|$ contains a neighborhood of $\inf _{\mathbb{R}}\left|U_{c_{*}}\right|$ for $c$ close to $c_{*}$. For $c_{*}=\mathfrak{c}_{s}$, this is no longer the case: we have only a one-sided neighborhood of $\inf _{\mathbb{R}}\left|U_{\mathfrak{c}_{S}}\right|$. It is plausible that the study for $\mu$ in this one-sided neighborhood of $\inf _{\mathbb{R}}\left|U_{\mathfrak{c}_{s}}\right|$ can be done as in the proof of Theorem 23, but, for the remaining values of $\mu$, we have to find a sharp ansatz, which is not very easy to find.

Furthermore, for the linear instability which is expected if $(d P / d c)_{\mid c=\mathfrak{c}_{s}}>0$, let us mention the following point. For the eigenvalue problem studied in [Benzoni-Gavage 2010b], the characteristic 
equation for the constant coefficient limit at infinity, namely

$$
r^{4}-\left(\mathfrak{c}_{s}^{2}-c_{*}^{2}\right) r^{2}-2 c_{*} \lambda r+\lambda^{2}=0
$$

becomes, when $c_{*}=\mathfrak{c}_{s}$,

$$
r^{4}-2 \mathfrak{c}_{s} \lambda r+\lambda^{2}=0
$$

The behavior of the roots for small $\lambda$ is then different from the case $0<c_{*}<\mathfrak{c}_{s}$. Indeed, there exists a root $\sim \lambda /\left(2 \mathfrak{c}_{s}\right)$ for $\lambda \rightarrow 0$, and, for the three other roots, we use the variable $r=\sqrt[3]{\lambda} z$, which transforms $r^{4}-2 \mathfrak{c}_{s} \lambda r+\lambda^{2}=0$ into $z^{4}-2 \mathfrak{c}_{s} z+\lambda^{2 / 3}=0$. This last equation has, for $\lambda \rightarrow 0$, three roots $\sim j k \sqrt[3]{2 \mathfrak{c}_{s}}$, where $j=\mathrm{e}^{2 i \pi / 3}$ and $k=0,1,2$. In particular, (53) has three roots $\sim j k \sqrt[3]{2 \mathfrak{c}_{s} \lambda}, k=0,1,2$. The value $\lambda=0$ is then a branching point, and we shall have a smooth problem not in $\lambda$ but in $\sqrt[3]{\lambda}$. Since analyticity is not necessary for our purpose, we may define an Evans function $\tilde{\mathbb{D}}$ in $\mathbb{R}_{+}$, smooth, and such that, for $\lambda>0, \tilde{\mathbb{D}}(\sqrt[3]{\lambda})=0$ if and only if $\lambda$ is an unstable eigenvalue for (27). Another difficulty comes from the fact that it will be difficult to find an analytic extension of the Evans function $\tilde{\mathbb{D}}$ near 0 since, by Proposition 2, for $c_{*}=\mathfrak{c}_{s}, u_{*}$ and $\eta_{*}$ decay only at an algebraic rate and not an exponential rate. Consequently, we can not use the gap lemma of [Gardner and Zumbrun 1998] and [Kapitula and Sandstede 1998]. Finally, as a straightforward computation shows, the stable and unstable subspaces for the eigenvalue problem are transverse for $\lambda>0$ but their continuous extensions at $\lambda=0$ have a nontrivial intersection. Therefore, both stability and instability require some further analysis, and the situation is then much more delicate than the one studied in Section 5A.

\section{Appendix A. Construction of a Liapounov functional in the stable case in the Grillakis-Shatah-Strauss framework}

We work with the notations of [Grillakis et al. 1987], and recall them briefly. We consider a Hamiltonian equation in a real Hilbert space $\mathscr{X}$, with scalar product $(\cdot, \cdot) \mathscr{X}$, under the form

$$
\frac{\partial u}{\partial t}=J E^{\prime}(u)
$$

where $J: \mathscr{X}^{*} \rightarrow \mathscr{X}$ is a closed linear operator with dense domain and skew-symmetric. Assume that $T$ is a $\mathscr{C}_{0}$-group of unitary operators in $\mathscr{X}$ generated by $T^{\prime}(0)$, which is skew-adjoint and with dense domain, and that $E$ is invariant by $T$; that is, $E(T(s) u)=E(u)$ for any $s \in \mathbb{R}, u \in \mathscr{X}$. Assume moreover that $T(s) J=J T(-s)^{*}$ for any $s \in \mathbb{R}$ and that there exists $B: \mathscr{X} \rightarrow \mathscr{X}^{*}$, linear and bounded, such that $B^{*}=B$ and $J B$ is an extension of $T^{\prime}(0)$. We then set

$$
Q(u) \equiv \frac{1}{2}\langle B u, u\rangle_{\mathscr{X}}, \mathscr{X} .
$$

The basic assumptions of [Grillakis et al. 1987] are the following ones.

Assumption 1 (existence of solutions). For any $r>0$ there exists $t_{*}>0$, depending only on $r$, such that, for any $u^{\text {in }} \in \mathscr{X}$, there exists a $u \in \mathscr{C}\left(\left(-t_{*}, t_{*}\right), \mathscr{X}\right)$ with $u(0)=u^{\text {in }}$ solution of $(\mathscr{H})$ in the sense that, for 
any $\varphi \in D(J) \subset \mathscr{L}^{*}$,

$$
\frac{d}{d t}\langle u(t), \varphi\rangle_{\mathscr{X}^{*}, \mathscr{X}}=-\left\langle E^{\prime}(u(t)), J \varphi\right\rangle_{\mathscr{X}^{*}, \mathscr{X}} \quad \text { in } \mathscr{D}^{\prime}\left(\left(-t_{*}, t_{*}\right)\right),
$$

and verifying $E(u(t))=E\left(u^{\mathrm{in}}\right)$ and $Q(u(t))=Q\left(u^{\mathrm{in}}\right)$ for $t \in\left(-t_{*}, t_{*}\right)$.

Assumption 2 (existence of "bound states"). There exists an interval $\Omega \subset \mathbb{R}$, not reduced to a singleton, and a mapping $\Omega \ni \omega \mapsto \phi_{\omega} \in \mathscr{L}$ of class $\mathscr{C}^{1}$ such that, for any $\omega \in \Omega$,

$$
E^{\prime}\left(\phi_{\omega}\right)=\omega Q^{\prime}\left(\phi_{\omega}\right), \quad \phi_{\omega} \in D\left(T^{\prime}(0)^{3}\right) \cap D\left(J I T^{\prime}(0)^{2}\right), \quad T^{\prime}(0) \phi_{\omega} \neq 0 .
$$

Assumption 3 (spectral decomposition). For each $\omega \in \Omega$, the operator $H_{\omega} \equiv E^{\prime \prime}\left(\phi_{\omega}\right)-\omega Q^{\prime \prime}\left(\phi_{\omega}\right): \mathscr{L} \rightarrow \mathscr{Q}^{*}$ has its kernel spanned by $T^{\prime}(0) \phi_{\omega}$, has one negative simple eigenvalue and the rest of its spectrum is positive and bounded away from zero.

Under Assumption 2, we consider some $\omega_{*} \in \Omega$ and the associated bound state $\phi_{\omega_{*}}$, and then define, for $M>0$, the functional

$$
\mathscr{L}_{\omega_{*}}(u) \equiv E(u)-\omega_{*} Q(u)+\frac{M}{2}\left(Q(u)-Q\left(\phi_{\omega_{*}}\right)\right)^{2} .
$$

It is clear that $\phi_{\omega_{*}}$ is a critical point of $\mathscr{L}_{\omega_{*}}: \mathscr{L}^{\prime}\left(\phi_{\omega_{*}}\right)=E^{\prime}\left(\phi_{\omega_{*}}\right)-\omega_{*} Q^{\prime}\left(\phi_{\omega_{*}}\right)=0$. We denote by

$$
\Lambda \equiv \mathscr{L}_{\omega_{*}}^{\prime \prime}\left(\phi_{\omega_{*}}\right)=H_{\omega_{*}}+M\left\langle Q^{\prime}\left(\phi_{\omega_{*}}\right), \cdot\right\rangle_{\mathscr{L} *, \mathscr{X}} Q^{\prime}\left(\phi_{\omega_{*}}\right)
$$

its second derivative, which is a self-adjoint operator. The main result of this appendix is the following. Theorem 26. We make Assumptions 2 and 3 and suppose that the operator $\left\langle Q^{\prime}\left(\phi_{\omega_{*}}\right), \cdot\right\rangle_{\mathscr{x}^{*}, \mathscr{X}} Q^{\prime}\left(\phi_{\omega_{*}}\right)$ is a compact perturbation of $H_{\omega_{*}}$. If $\left(d Q\left(\phi_{\omega}\right) / d \omega\right)_{\mid \omega=\omega_{*}}<0$ and

$$
M>\frac{1}{-\frac{d Q\left(\phi_{\omega}\right)}{d \omega} \mid \omega=\omega_{*}},
$$

there exists $\delta>0$ such that

$$
\langle\Lambda v, v\rangle \geq \delta\|v\|^{2} \quad \text { for all } v \in X \quad \text { s.t. }\left(v, T^{\prime}(0) \phi_{\omega_{*}}\right) \mathscr{X}=0 .
$$

In particular, for any $u \in X$ with $\inf _{s \in \mathbb{R}}\left\|u-T(s) \phi_{\omega_{*}}\right\|^{2} \leq \epsilon$, we have

$$
\inf _{s \in \mathbb{R}}\left\|u-T(s) \phi_{\omega_{*}}\right\|^{2} \leq \frac{2}{\delta}\left(\mathscr{L}(u)-\mathscr{L}\left(\phi_{\omega_{*}}\right)\right) .
$$

Therefore, when Assumption 1 is moreover satisfied, the (global) solution $u(t)$ to $(\mathscr{H})$ with initial datum $u^{\text {in }}$ satisfies

$$
\sup _{t \in \mathbb{R}} \inf _{s \in \mathbb{B}}\left\|u(t)-T(s) \phi_{\omega_{*}}\right\|^{2} \leq \frac{2}{\delta}\left(\mathscr{L}\left(u^{\mathrm{in}}\right)-\mathscr{L}\left(\phi_{\omega_{*}}\right)\right) \leq K\left\|u^{\mathrm{in}}-\phi_{\omega_{*}}\right\|^{2},
$$

provided the right-hand side is sufficiently small. 
We point out that the condition that the operator $\left\langle Q^{\prime}\left(\phi_{\omega_{*}}\right), \cdot\right\rangle_{\mathscr{X}^{*}, \mathscr{X}} Q^{\prime}\left(\phi_{\omega_{*}}\right)$ is a compact perturbation of $H_{\omega_{*}}$ is not very restrictive, since, in many cases coming from PDEs, it involves less derivatives than $H_{\omega_{*}}$ and $Q^{\prime}\left(\phi_{\omega_{*}}\right)$ tends to zero at spatial infinity.

This type of Liapounov functional has been used in [Barashenkov 1996] to prove that the traveling waves of (NLS) in dimension one are stable when $d P / d c<0$. The proof follows basically the one in [Barashenkov 1996], but some points have to be clarified. The interest of this type of Liapounov functional is that the saddle point $\phi_{\omega_{*}}$ is now a nondegenerate local minimum for $\mathscr{L}_{\omega_{*}}$. This is a great advantage for numerical simulation of the "bound states", since a gradient flow method on $\mathscr{L}_{\omega_{*}}$ can be used. This approach has been used, with a very similar functional, by N. Papanicolaou and P. Spathis [1999] for the numerical simulation of the traveling waves for a planar ferromagnets model. In the same spirit, in [Chiron and Scheid 2012], we also use a gradient flow method on this type of functional for the numerical simulation of the traveling waves for (NLS) in two dimensions.

Proof of Theorem 26. Recall that the spectrum of $H_{\omega_{*}}$ is, by Assumption 3, such that $-\lambda_{*}^{2} \in \sigma\left(H_{\omega_{*}}\right)$, $0 \in \sigma\left(H_{\omega_{*}}\right)$ and $\sigma\left(H_{\omega_{*}}\right) \backslash\left\{-\lambda_{*}^{2}, 0\right\} \subset[\delta,+\infty)$ for some $\delta>0$. Since we assume that $\left\langle Q^{\prime}\left(\phi_{\omega_{*}}\right), \cdot\right\rangle Q^{\prime}\left(\phi_{\omega_{*}}\right)$ is a compact perturbation of $H_{\omega_{*}}$, the essential spectrum of $\Lambda$ is the same as the one of $H_{\omega_{*}}$, and hence is included in $[\delta,+\infty)$. Furthermore, $0 \in \sigma\left(H_{\omega_{*}}\right)$ and $\operatorname{ker}\left(H_{\omega_{*}}\right)=\mathbb{R} T^{\prime}(0) \phi_{\omega_{*}}$ by Assumption 3. Since $Q^{\prime}\left(\phi_{\omega_{*}}\right)=B \phi_{\omega_{*}}$ and $J B$ is an extension of $T^{\prime}(0)$, we have that $\left\langle Q^{\prime}\left(\phi_{\omega_{*}}\right), T^{\prime}(0) \phi_{\omega_{*}}\right\rangle \mathscr{x}^{*}, \mathscr{X}=$ $\left\langle B \phi_{\omega_{*}}, J B \phi_{\omega_{*}}\right\rangle_{\mathscr{X}^{*}, \mathscr{L}}=0$; hence $\Lambda\left(T^{\prime}(0) \phi_{\omega_{*}}\right)=0$. Noticing that $\left\langle Q^{\prime}\left(\phi_{\omega_{*}}\right), \cdot\right\rangle_{\mathscr{X}^{*}, \mathscr{L}} Q^{\prime}\left(\phi_{\omega_{*}}\right)$ is a nonnegative operator, we infer that $\operatorname{ker}(\Lambda)=\operatorname{ker}\left(H_{\omega_{*}}\right)=\mathbb{R} T^{\prime}(0) \phi_{\omega_{*}}$ is one-dimensional. Therefore, it suffices to show that $\Lambda$ has no eigenvalues in $(-\infty, 0)$. As we have seen that $\left\langle Q^{\prime}\left(\phi_{\omega_{*}}\right), \cdot\right\rangle_{X^{*}, X} Q^{\prime}\left(\phi_{\omega_{*}}\right)$ is a nonnegative operator, we deduce that $\sigma(\Lambda) \subset\left[-\lambda_{*}^{2},+\infty\right)$. Let us first show that $-\lambda_{*}^{2} \notin \sigma(\Lambda)$ by contradiction. If $-\lambda_{*}^{2}$ is an eigenvalue of $\Lambda$, then there exists $v \in X, v \neq 0$, such that $0=$ $\left(\Lambda+\lambda_{*}^{2}\right) v=\left(H+\lambda_{*}^{2}\right) v+M\left\langle Q^{\prime}\left(\phi_{\omega_{*}}\right), v\right\rangle_{\mathscr{C}^{*}, \mathscr{X}} Q^{\prime}\left(\phi_{\omega_{*}}\right)$. Taking the duality product with $v$ yields $0=\left\langle\left(H_{\omega_{*}}+\lambda_{*}^{2}\right) v, v\right\rangle_{\mathscr{L}^{*}, \mathbb{B} X}+M\left\langle Q^{\prime}\left(\phi_{\omega_{*}}\right), v\right\rangle_{\mathscr{W}^{*}, \mathscr{X}}^{2}$. Since the two terms in the sum are nonnegative, this implies $\left\langle Q^{\prime}\left(\phi_{\omega_{*}}\right), v\right\rangle_{\mathscr{C}^{*}, \mathscr{X}}=0$ and $\left\langle\left(H_{\omega_{*}}+\lambda_{*}^{2}\right) v, v\right\rangle_{\mathscr{X}^{*}, \mathscr{X}}=0$, which in turn implies $v \in \operatorname{ker}\left(H_{\omega_{*}}+\lambda_{*}^{2}\right)=\mathbb{R} \chi$ (here, $\chi$ is a negative eigenvector of $H_{\omega_{*}}$ for the eigenvalue $-\lambda_{*}^{2}<0$ ). As a consequence, we must have $\left\langle Q^{\prime}\left(\phi_{\omega_{*}}\right), \chi\right\rangle_{\mathscr{X}^{*}, \mathscr{X}}=0$. On the other hand, differentiating the equality $E^{\prime}\left(\phi_{\omega}\right)-\omega Q^{\prime}\left(\phi_{\omega}\right)=0$ at $\omega=\omega_{*}$ yields $Q^{\prime}\left(\phi_{\omega_{*}}\right)=H_{\omega_{*}} \phi^{\prime}$, where $\phi^{\prime} \equiv(d \phi / d \omega)_{\mid \omega=\omega_{*}}$. Thus we must have $0=\left\langle H_{\omega_{*}} \phi^{\prime}, \chi\right\rangle_{\mathscr{X}^{*}, \mathscr{X}}=$ $\left\langle H_{\omega_{*}} \chi, \phi^{\prime}\right\rangle_{\mathscr{C}^{*}, \mathscr{X}}=-\lambda_{*}^{2}\left(\chi, \phi^{\prime}\right)$. Therefore, $\phi^{\prime}$ is orthogonal to $\chi$ and this gives $\left\langle H_{\omega_{*}} \phi^{\prime}, \phi^{\prime}\right\rangle_{\mathscr{C}^{*}, \mathscr{X}} \geq 0$. However, this is not possible if $\left(d Q\left(\phi_{\omega}\right) / d \omega\right)_{\mid \omega=\omega_{*}}<0$, since $\left(d Q\left(\phi_{\omega}\right) / d \omega\right)_{\mid \omega=\omega_{*}}=-\left\langle H_{\omega_{*}} \phi^{\prime}, \phi^{\prime}\right\rangle_{\mathscr{X}^{*}, \mathscr{X}}$. As a consequence, if $\lambda$ is a negative element of the spectrum of $\Lambda$, then $-\lambda_{*}^{2}<\lambda<0$ and $\lambda$ is an eigenvalue: there exists $v \in X$ such that $v \neq 0$ and

$$
\lambda v=\Lambda v=H_{\omega_{*}} v+M\left\langle Q^{\prime}\left(\phi_{\omega_{*}}\right), v\right\rangle_{\mathscr{X}^{*}, \mathscr{L}} Q^{\prime}\left(\phi_{\omega_{*}}\right) .
$$

Since $-\lambda_{*}^{2}<\lambda<0$, we then infer

$$
v=-M\left\langle Q^{\prime}\left(\phi_{\omega_{*}}\right), v\right\rangle_{\mathscr{Q} * \mathscr{Q}}\left(H_{\omega_{*}}-\lambda\right)^{-1} Q^{\prime}\left(\phi_{\omega_{*}}\right) .
$$


Since $v \neq 0$, we can not have $\left\langle Q^{\prime}\left(\phi_{\omega_{*}}\right), v\right\rangle_{\mathscr{L}^{*}, \mathscr{X}}=0$. Then, taking the scalar product of (A-1) with $\square^{-1} Q^{\prime}\left(\phi_{\omega_{*}}\right)$ (here, $\square: \mathscr{X} \rightarrow \mathscr{X}^{*}$ is the usual Riesz isomorphism) gives

$$
g(\lambda)=0, \quad \text { where } g(t) \equiv 1+M\left(\left(H_{\omega_{*}}-t\right)^{-1} Q^{\prime}\left(\phi_{\omega_{*}}\right), \square^{-1} Q^{\prime}\left(\phi_{\omega_{*}}\right)\right)_{\mathscr{Q}}, \quad-\lambda_{*}^{2}<t<0 .
$$

It is clear that $g$ is smooth in $\left(-\lambda_{*}^{2}, 0\right)$ and that

$$
g^{\prime}(t)=M\left(\left(H_{\omega_{*}}-t\right)^{-2} Q^{\prime}\left(\phi_{\omega_{*}}\right), \square^{-1} Q^{\prime}\left(\phi_{\omega_{*}}\right)\right)_{\mathscr{Q}}=M\left\|\left(H_{\omega_{*}}-t\right)^{-1} Q^{\prime}\left(\phi_{\omega_{*}}\right)\right\|_{\mathscr{X}}^{2}>0 .
$$

We now study the limit of $g$ at $0^{-}$. Let us recall that $H_{\omega_{*}} \phi^{\prime}=Q^{\prime}\left(\phi_{\omega_{*}}\right)$ and that we have already seen that $\left\langle Q^{\prime}\left(\phi_{\omega_{*}}\right), T^{\prime}(0) \phi_{\omega_{*}}\right\rangle_{\mathscr{X} *, \mathscr{X}}=0$; i.e., $\square^{-1} Q^{\prime}\left(\phi_{\omega_{*}}\right)$ is orthogonal to $\operatorname{ker}\left(H_{\omega_{*}}\right)$. Therefore, as $t \rightarrow 0^{-}$,

$$
\left(\left(H_{\omega_{*}}-t\right)^{-1} Q^{\prime}\left(\phi_{\omega_{*}}\right), \square^{-1} Q^{\prime}\left(\phi_{\omega_{*}}\right)\right) \rightarrow\left(\phi^{\prime}, \square^{-1} Q^{\prime}\left(\phi_{\omega_{*}}\right)\right)=\left\langle Q^{\prime}\left(\phi_{\omega_{*}}\right), \phi^{\prime}\right\rangle_{\mathscr{X}^{*}, \mathscr{X}}={\frac{d Q\left(\phi_{\omega}\right)}{d \omega}}_{\mid \omega=\omega_{*}}
$$

and thus

$$
g(t) \rightarrow 1+M \frac{d Q\left(\phi_{\omega}\right)}{d \omega} \mid \omega=\omega_{*} \quad \text { as } t \rightarrow 0^{-} .
$$

Since $\left(d Q\left(\phi_{\omega}\right) / d \omega\right)_{\mid \omega=\omega_{*}}<0$ by hypothesis, it follows that, if $M>-1 /\left(d Q\left(\phi_{\omega}\right) / d \omega\right)_{\mid \omega=\omega_{*}}>0$, the function $g$ increases in $\left(-\lambda_{*}^{2}, 0\right)$ and tends to some negative limit at $0^{-}$. In particular, $g$ is negative; hence we can not have $g(\lambda)=0$ with $\lambda \in\left(-\lambda_{*}^{2}, 0\right)$. We have therefore shown that the spectrum of $\Lambda$ consists in a simple eigenvalue 0 with eigenspace spanned by $T^{\prime}(0) \phi_{\omega_{*}}$ and the rest of the spectrum is positive and bounded away from 0 . This concludes the proof.

We would like to point out the fact that, in the proof of [Barashenkov 1996], $-\lambda_{*}^{2} \notin \sigma(\Lambda)$ was not shown, the kernel of $\Lambda$ was not studied and the essential spectrum was not considered. Moreover, the functional spaces are not given; hence we do not know for which perturbations stability holds.

\section{Appendix B. From linear to nonlinear instability}

We still consider in this appendix an abstract Hamiltonian equation in the framework of [Grillakis et al. 1987]

$$
\frac{\partial u}{\partial t}=J E^{\prime}(u)
$$

on the real Hilbert space $\mathscr{X}$, with scalar product $(\cdot, \cdot) \mathscr{X}$. Here $E: \mathscr{X} \rightarrow \mathbb{R}$ is of class $\mathscr{C}^{2}$ and $J: \mathscr{X}^{*} \rightarrow \mathscr{X}$ is a closed linear operator with dense domain and skew-symmetric in the sense that $(u, J w) \mathscr{L}=-\langle w, J u\rangle_{\mathscr{L}}$, $\mathscr{L}$ for $u \in \mathscr{X}, w \in \mathscr{X}^{*}$.

We assume that there exists a $\mathscr{C}_{0}$-group $T$ of unitary operators in $\mathscr{X}$ generated by $T^{\prime}(0)$, which is skew-adjoint and with dense domain, and that $E$ is invariant by $T$; that is, $E(T(\omega) u)=E(u)$ for any $\omega \in \mathbb{R}, u \in \mathscr{X}$. Assume moreover that $T(\omega) J=J T(-\omega)^{*}$ for any $\omega \in \mathbb{R}$ and that there exists $B: \mathscr{X} \rightarrow \mathscr{X}^{*}$, linear and bounded, such that $B^{*}=B$ and $J B$ is an extension of $T^{\prime}(0)$. We then set

$$
Q(u) \equiv \frac{1}{2}\langle B u, u\rangle_{\mathscr{X}^{*}, \mathscr{X}},
$$


which is invariant by the flow ( $\mathscr{H}$ ) (see [Grillakis et al. 1987]). By "bound state", we mean a particular solution $U$ of $(\mathscr{H})$ of the form $U(t)=T(\omega t) \phi$ for some $\omega \in \mathbb{R}$ and where $\phi \in \mathscr{X}, \phi \neq 0$. In other words, $E^{\prime}(\phi)=\omega Q^{\prime}(\phi)$.

There exist an open interval $\Omega \subset \mathbb{R}$, not reduced to a singleton, and a mapping $\Omega \ni \omega \mapsto \phi_{\omega} \in X$ of class $\mathscr{C}^{1}$ such that, for any $\omega \in \Omega$,

$$
E^{\prime}\left(\phi_{\omega}\right)=\omega Q^{\prime}\left(\phi_{\omega}\right), \quad \phi_{\omega} \in D\left(T^{\prime}(0)^{3}\right) \cap D\left(J I T^{\prime}(0)^{2}\right), \quad T^{\prime}(0) \phi_{\omega} \neq 0 .
$$

The solution $U(t)=T(\omega t) \phi$ is said to be stable in $\mathscr{X}$ if, for any $\varepsilon>0$, there exists $\delta>0$ such that

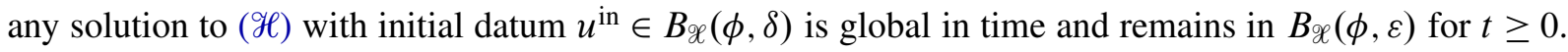
Otherwise, it is said to be unstable. This supposes some knowledge of the Cauchy problem for ( $\mathscr{H}$ ) (at least existence of solutions). If we are given some Banach space $\mathscr{Y} \supset \mathscr{X}$ with continuous imbedding $\mathscr{L} \hookrightarrow \mathscr{Y}$, we may also say that the solution $U(t)=T(\omega t) \phi$ is said to be stable from $\mathscr{X}$ to $\mathscr{Y}$ if, for any $\varepsilon>0$, there exists $\delta>0$ such that any solution to $(\mathscr{H})$ with initial datum $u^{\text {in }} \in B_{\mathscr{L}}(\phi, \delta)$ remains in $B_{\mathscr{y}}(\phi, \varepsilon)$ for $t \geq 0$. Clearly, a solution stable in $\mathscr{X}$ is precisely a solution stable from $\mathscr{X}$ to $\mathscr{X}$, and is also stable from $\mathscr{X}$ to $\mathscr{Y}$; hence instability from $\mathscr{X}$ to $\mathscr{Y}$ is a stronger statement that instability in $\mathscr{X}$.

In our framework, the notion of orbital stability is more relevant. Let us consider $\mathbb{G}$ a group and $\mathbb{T}: \mathbb{R} \times \mathbb{G} \rightarrow \mathscr{G}_{\mathcal{C}}(\mathscr{X})$ a unitary representation of $\mathbb{R} \times \mathbb{G}$ on $\mathscr{X}$, extending $T: \mathbb{R} \rightarrow \mathscr{X}$ and leaving $E$ and $Q$ invariant. Then, $U(t)=T(\omega t) \phi$ is said to be orbitally stable in $\mathscr{X}$ (for the group $\mathbb{G}$ ) if, for any $\varepsilon>0$, there exists $\delta>0$ such that any solution to $(\mathscr{H})$ with initial datum $u^{\text {in }} \in B(\phi, \delta)$ is global in time and remains in $\bigcup_{(\omega, g) \in \mathbb{R} \times \mathbb{G}} B(\mathbb{T}(\omega, g) \phi, \varepsilon)$ for $t \geq 0$. We may also define orbital instability from $\mathscr{X}$ to $\mathscr{Y} \supset \mathscr{X}$ in a natural way.

In [Grillakis et al. 1987; 1990], a general framework for the stability analysis for the "bound state" has been given. In particular, the nonlinear orbital instability is proved in [Grillakis et al. 1987] through the construction of a Liapounov-type functional. However, this method does not give a clear understanding neither of how we get farther from the "bound state", nor on which timescale it occurs.

The need for allowing an additional group of invariances $\mathbb{G}$ can be seen in the case of bound state solutions, that is $U(t)=\mathrm{e}^{i \omega t} \phi_{\omega}$, to the nonlinear Schrödinger equation

$$
i \partial_{t} \Psi+\Delta \Psi+\Psi f\left(|\Psi|^{2}\right)=0,
$$

or the nonlinear Klein-Gordon equation in $\mathbb{R}^{d}$

$$
\partial_{t}^{2} \Psi=\Delta \Psi+\Psi f\left(|\Psi|^{2}\right),
$$

since, then, the invariance by translation in space must be taken into account in the definition of orbital stability, and we are in a case where $\mathbb{G}=\mathbb{R}^{d}$ acts naturally by translation. The translations are taken into account in [Cazenave and Lions 1982]. In [Grillakis et al. 1987; 1990], the notion of orbital stability is for $\mathbb{G}$ trivial. It is clear from the definition that orbital stability for $\mathbb{G}=\{0\}$ implies orbital stability for arbitrary $\mathbb{G}$. For the instability in the nonlinear Schrödinger equation or the nonlinear Klein-Gordon equation, [Grillakis et al. 1987] and [Shatah and Strauss 1985] work with radial $H^{1}$ functions. The fact 
that this also implies the orbital instability with the action of $\mathbb{G}=\mathbb{R}^{d}$ by translations follows immediately from the fact that for any $\theta \in[0,2 \pi]$ the manifold $\mathfrak{M}_{\theta} \equiv\left\{\mathrm{e}^{i \theta} \phi(\cdot-y), y \in \mathbb{R}^{d}\right\}$ is orthogonal to $H_{\text {rad }}^{1}\left(\mathbb{R}^{d}\right)$.

For the stability analysis of a "bound state" $U(t)=T\left(\omega_{*} t\right)\left(\phi_{\omega_{*}}\right)$, it is natural to consider the linearization of ( $\mathscr{H}$ ) near $\phi$. More precisely, we linearize according to the ansatz $u(t)=T\left(\omega_{*} t\right)\left(\phi_{\omega_{*}}+v(t)\right)$, so that the "bound state" becomes stationary. The linearized problem then becomes

$$
\frac{\partial v}{\partial t}=J\left(E^{\prime \prime}(\phi)-\omega Q^{\prime \prime}(\phi)\right) v=\mathscr{L} \mathscr{L} v,
$$

where, $\mathbb{\square}: \mathscr{X} \rightarrow \mathscr{L}^{*}$ denoting the Riesz isomorphism, $\mathscr{E} \equiv J \square: \mathscr{X} \rightarrow \mathscr{X}$ is skew-adjoint.

The purpose of this appendix is to give a general result, for Hamiltonian equations, showing that linear instability implies nonlinear (orbital) instability. By linear instability, we mean that the complexification of $[\mathscr{E} \mathscr{L}]_{\mathbb{C}}$ has at least one eigenvalue in the right half-space $\{\operatorname{Re}>0\}$. The argument follows ideas from the works of F. Rousset and N. Tzvetkov [2008; 2009].

Showing the existence of an unstable eigenvalue can be done through various techniques: see [Grillakis et al. 1990] (in the framework of [Grillakis et al. 1987] when $J$ is onto), [Grillakis 1988] (assuming a special structure of the Hamiltonian equation); for uses of the Vakhitov-Kolokolov function, see [de Bouard 1995], [Di Menza and Gallo 2007] or [Pelinovsky and Kevrekidis 2008]. When $J$ is not onto, we quote [Lopes 2002]. For one-dimensional partial differential equations, one may also use the Evans function (see the survey [Sandstede 2002]) as in [Pego and Weinstein 1992; Gardner and Zumbrun 1998; Kapitula and Sandstede 1998; Zumbrun 2008]. The paper [Lin 2008] proposes another approach which allows treating pseudodifferential equations, such as the BBM equation, the Benjamin-Ono equation, regularized Boussinesq equations, the intermediate long wave equation, etc.

In order to pass from linear to nonlinear instability, the following result is standard. We refer to the paper by D. Henry, J. Perez and W. Wreszinski [Henry et al. 1982]. It can also be found in [Grillakis 1988; Shatah and Strauss 2000].

Theorem B.1 [Henry et al. 1982; Grillakis 1988; Shatah and Strauss 2000]. We assume that A generates a continuous semigroup on $X$ and that $\sigma(\mathscr{A})$ meets the right half-space $\{\operatorname{Re}>0\}$. We assume moreover that $F: X \rightarrow X$ is locally Lipschitz continuous and satisfies, for some $\alpha>0,\|F(v)\|_{X}=\mathcal{O}\left(\|v\|_{X}^{1+\alpha}\right)$ as

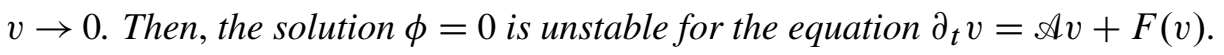

In [Shatah and Strauss 2000], it is claimed that an orbital instability result can also be established. Theorem B.1 shows nonlinear instability without assuming that the equation is Hamiltonian. However, if $\left(\mathscr{H}_{\text {lin }}\right)$ can be solved using a semigroup, it does not give the growth of its norm. Moreover, it does not say that, if the initial datum is in a most unstable direction, that is, an eigendirection of $\mathscr{A}$ corresponding to an eigenvalue of maximal positive real part (plus the complex conjugate if necessary), then one can track the exponential growth of the solution. In particular, it does not explain the mechanism of instability and does not give any information on the timescale on which one see the instability. For instance, some strong instability results are shown by proving blow-up in finite time (see [Berestycki and Cazenave 1981]), but the instability due to an exponentially growing mode holds on a much smaller timescale. 
We wish to provide here some results clarifying the instability mechanism by tracking the exponentially growing mode.

A spectral mapping theorem for linearized Hamiltonian equations. When we want to prove a nonlinear instability result from a linear instability one, we need some information on the growth of the semigroup $\mathscr{L} \mathscr{L}$, when such a semigroup $\mathrm{e}^{t \mathscr{L} \mathscr{L}}$ exists, which we shall assume in this appendix. The growth estimate on $e^{t \mathscr{L L}}$ relies classically on the following spectral mapping result due to J. Prüss [1984], which generalizes the work of L. Gearhart [1978].

Theorem B.2 [Prüss 1984]. Let $X$ be a complex Hilbert space and $\mathscr{A}$ an unbounded operator on $X$ which generates a continuous semigroup $\mathrm{e}^{t \mathfrak{A}}$ on $X$. For $t \in(0,+\infty)$, we have

$$
\sigma\left(\mathrm{e}^{t \not \mathcal{A}}\right) \backslash\{0\}=\left\{\mathrm{e}^{\lambda t} \text {, either }\left(\lambda+\frac{2 i \pi}{t} \mathbb{Z}\right) \cap \sigma(\mathscr{A}) \neq \varnothing, \text { or } \sup _{k \in \mathbb{Z}}\left\|\left(\mathscr{A}-\lambda-\frac{2 i \pi k}{t}\right)^{-1}\right\|_{\mathscr{L}_{C}(X)}=+\infty\right\} .
$$

The following result is an immediate corollary.

Corollary B.3. Let $X$ be a complex Hilbert space and $A$ an unbounded operator on $X$ which generates a continuous semigroup $\mathrm{e}^{t \mathfrak{A}}$ on $X$. Assume that, for any $\gamma \in \mathbb{R}^{*}$, we have

$$
\limsup _{|\tau| \rightarrow+\infty}\left\|(\mathscr{A}-\gamma-i \tau)^{-1}\right\|_{\mathscr{L}_{c}(X)}<+\infty,
$$

and that there exists $\vartheta_{0} \in[0,+\infty)$ such that $\sigma_{\mathrm{ess}}(\mathscr{A})=\left\{i \vartheta, \vartheta \in \mathbb{R},|\vartheta| \geq \vartheta_{0}\right\}$. Then, for any $t \in(0,+\infty)$, the spectral mapping holds: $\sigma\left(\mathrm{e}^{t \mathscr{A}}\right) \backslash\{0\}=\mathrm{e}^{t \sigma(\mathscr{A})}$.

Proof. Since $\sigma_{\text {ess }}(\mathscr{A})=\left\{i \vartheta, \vartheta \in \mathbb{R},|\vartheta| \geq \vartheta_{0}\right\}$, we have $\mathbb{S}^{1} \subset \mathrm{e}^{t \sigma(\mathscr{A})} \subset \sigma\left(\mathrm{e}^{t \mathscr{A}}\right)$. If $\lambda \in \mathbb{C}$ does not have modulus one, then note that, when $(\lambda+(2 i \pi / t) \mathbb{Z}) \cap \sigma(\mathscr{A})=\varnothing$, the supremum for $k \in \mathbb{Z}$ in Theorem B.2 can be $+\infty$ only when $|k| \rightarrow+\infty$, and we conclude with our hypothesis.

The fact that we exclude 0 in the spectral mapping theorem just comes from the fact that we consider a semigroup and not a group. However, in most Hamiltonian PDEs, we have time reversibility and we have actually a continuous group and not only a semigroup. In most cases, we work with $A: D(A) \subset Y \rightarrow Y$ where $Y$ is a real Hilbert space, thus for applying Theorem B.2 or Corollary B.3 we have to consider, as usual, the complexified operator $A_{\mathbb{C}}: D\left(A_{\mathbb{C}}\right) \equiv D(A) \oplus i D(A) \subset Y_{\mathbb{C}} \equiv Y \oplus i Y \rightarrow Y_{\mathbb{C}}$ defined by $A_{\mathbb{C}}(u+i v)=A u+i A v$.

It seems that the first time Theorem B.2 is used to prove a growth estimate on a semigroup was by T. Kapitula and B. Sandstede [1998]. Later, F. Gesztesy et al. [2000] also used this result for bound states for (NLS). The bounds on the resolvent in [Kapitula and Sandstede 1998] were proved using the particular structure of the linearized operator. In [Gesztesy et al. 2000], the computations are more involved and rely on suitable kernel estimates of some Hilbert-Schmidt operators. The same type of estimates have also been used in [Di Menza and Gallo 2007].

The main objective of this appendix is to provide a generalization of these results to a wide class of Hamiltonian equations. Indeed, the approaches in [Kapitula and Sandstede 1998; Gesztesy et al. 2000] seem specific to the problem. In addition, it is not clear whether the computations in [Gesztesy et al. 
2000; Di Menza and Gallo 2007] can be extended to other types of equations. In particular, in [Chiron 2012] and in the present paper, we have a situation similar to the one studied in [Di Menza and Gallo 2007], namely traveling wave solutions to a nonlinear Schrödinger equation with nonzero condition at infinity, but, for nonzero propagation speeds, the traveling wave is not real-valued (as it is in [Di Menza and Gallo 2007] for stationary waves or for bound state solutions), and the block diagonal structure of the linearized Hamiltonian disappears. An additional difficulty is that, in [Chiron 2012] and the present work, the limits of the traveling waves at $+\infty$ and $-\infty$ differ.

The proof we give is based on ideas from [Rousset and Tzvetkov 2008; 2009] and makes very few spectral assumptions on $\mathscr{L}$.

Assumption A. The spectrum of $\mathscr{L}$ consists in a finite number (possibly zero) of nonpositive eigenvalues $-\mu_{1}, \ldots,-\mu_{q}$ in $(-\infty, 0]$, each one with finite multiplicity, and the rest of the spectrum is positive and bounded away from 0 . Furthermore, for any $1 \leq k \leq q$, we have $\operatorname{ker}\left(\mathscr{L}+\mu_{k}\right) \subset D(\mathscr{F})$ and $\mathscr{L}\left[\operatorname{ker}\left(\mathscr{L}+\mu_{k}\right)\right] \subset D(\mathscr{L})$. Finally, there exists $\vartheta_{0} \in[0,+\infty)$ such that $\sigma_{\text {ess }}\left(\mathscr{L} \mathscr{L}=\left\{i \vartheta, \vartheta \in \mathbb{R},|\vartheta| \geq \vartheta_{0}\right\}\right.$.

The first hypothesis on the location of the spectrum of $\mathscr{L}$ is quite weak, since it is satisfied when $\mathscr{L}$ is bounded from below and has essential spectrum positive and bounded away from zero. Indeed, if $\delta>0$ is such that $\sigma_{\text {ess }}(\mathscr{L}) \subset[2 \delta,+\infty)$, then the eigenvalues of $\mathscr{L}$ in $(-\infty, \delta]$ are isolated, of finite multiplicity, and are bounded from below by assumption. The second hypothesis $\operatorname{ker}\left(\mathscr{L}+\mu_{k}\right) \subset D(\mathscr{L} \mathscr{\mathscr { S }})$ is a regularity assumption on the eigenvectors.

Let us recall that Theorem 25 ensures that the number of eigenvalues (with algebraic multiplicities) of $\mathscr{L} \mathscr{L}$ in the right half-space $\{\operatorname{Re}>0\}$ is less than or equal to the number of negative eigenvalues of $\mathscr{L}$, and hence is finite under Assumption A. Let us now state our main result, the proof of which is given starting on page 1413 .

Theorem B.4. We make Assumption A and suppose that $\mathscr{L} \mathscr{L}$ generates a continuous semigroup. Then, for any $t \in(0,+\infty)$, the spectral mapping holds: $\sigma\left(\mathrm{e}^{t[\mathscr{\mathscr { L }}]_{\mathbb{C}}}\right) \backslash\{0\}=\mathrm{e}^{t \sigma\left([\mathscr{\mathscr { L }}]_{\mathbb{C}}\right)}$. Furthermore, defining

$$
\gamma_{0} \equiv \sup \left\{\operatorname{Re}(\lambda), \lambda \in \sigma\left([\mathscr{L} \mathscr{L}]_{\mathbb{C}}\right) \cap\{\operatorname{Re} \geq 0\}\right\} \in[0,+\infty),
$$

for any $\beta>0$, there exists $M(\beta)>0$ such that, for any $t \geq 0$, we have

$$
\left\|\mathrm{e}^{t \mathscr{\mathscr { L }}}\right\|_{\mathscr{L}_{c}(\mathscr{X})} \leq M(\beta) \mathrm{e}^{\left(\gamma_{0}+\beta\right) t} .
$$

Assume in addition $\gamma_{0}>0$ and define

$$
m \equiv \max \left\{\text { algebraic multiplicity of } \lambda, \lambda \in \sigma\left([\mathscr{L}]_{\mathbb{C}}\right) \text { s.t. } \operatorname{Re} \lambda=\gamma_{0}\right\} \in \mathbb{N}^{*} \text {. }
$$

Then, there exists $M_{0}>0$ such that, for any $t \geq 0$, we have

$$
\left\|\mathrm{e}^{t \mathscr{\mathscr { L }}}\right\|_{\mathscr{L}_{c}(\mathscr{X})} \leq M_{0}(1+t)^{m-1} \mathrm{e}^{\gamma_{0} t}
$$

In particular, Theorem B.4 provides a very simple proof of the spectral mapping theorem used in [Gesztesy et al. 2000; Di Menza and Gallo 2007]. Indeed, the self-adjoint operator $\mathscr{L}$ involved in these 
papers is block diagonal:

$$
\mathscr{L}=\left(\begin{array}{cc}
\mathscr{L}_{1} & 0 \\
0 & \mathscr{L}_{2}
\end{array}\right)
$$

and both $\mathscr{L}_{1}, \mathscr{L}_{2}$ have at most two nonnegative eigenvalues. More generally, if $\mathscr{L}_{1}$ and $\mathscr{L}_{2}$ are closed self-adjoint operators on $X$ verifying Assumption A and if $\mathcal{N}: X \rightarrow X$ is a linear bounded operator which is compact with respect to $\mathscr{L}_{1}$ and $\mathscr{L}_{2}$, then the self-adjoint operator

$$
\mathscr{L}=\left(\begin{array}{ll}
\mathscr{L}_{1} & \mathcal{N} \\
\mathcal{N}^{*} & \mathscr{L}_{2}
\end{array}\right)
$$

also satisfies Assumption A. Indeed, $\mathscr{L}$ is bounded from below (since $\mathcal{N}$ is bounded) and its essential spectrum is $\sigma_{\text {ess }}\left(\mathscr{L}_{1}\right) \cup \sigma_{\text {ess }}\left(\mathscr{L}_{2}\right) \subset[\delta,+\infty)$ for some positive $\delta$, since $\mathcal{N}$ is compact with respect to $\mathscr{L}_{1}$ and $\mathscr{L}_{2}$. In [Kapitula and Sandstede 1998, Section 7.1; Georgiev and Ohta 2012, Proposition 10], a spectral mapping theorem is used for such an operator. In [Kapitula and Sandstede 1998], the specific algebra of the problem was used, and for [Georgiev and Ohta 2012], the proof relies on the arguments in [Gesztesy et al. 2000], but here again, in both cases, we may use Theorem B.4 to show the same result.

\section{Passing from linear to nonlinear instability.}

Semilinear type models. We start with a classical result for "semilinear" equations, proved on page 1416.

Theorem B.5. Let $X$ be a real Hilbert space, and consider an evolution equation of the form

$$
\frac{d v}{d t}=A d v+\Phi(v)
$$

where $\Phi: X \rightarrow X$ is a locally Lipschitz mapping satisfying $\Phi(v)=\mathscr{O}\left(\|v\|_{X}^{2}\right)$ as $v \rightarrow 0$ and $\mathscr{A}$ is a linear operator which generates a semigroup. We assume that $\mathscr{A}_{\mathbb{C}}: D\left(\mathscr{A}_{\mathbb{C}}\right) \subset X_{\mathbb{C}} \rightarrow X_{\mathbb{C}}$ has an unstable eigenvalue in the right half-plane $\{\operatorname{Re}>0\}$ and a finite number of eigenvalues in $\{\operatorname{Re}>0\}$. We define

$$
\gamma_{0} \equiv \sup \left\{\operatorname{Re}(\mu), \mu \in \sigma\left([\mathscr{\mathscr { L }}]_{\mathbb{C}}\right) \cap\{\operatorname{Re}>0\}\right\} \in(0,+\infty)
$$

and fix $\lambda \in \sigma\left(\mathscr{A}_{\mathbb{C}}\right)$ with $\operatorname{Re}(\lambda)=\gamma_{0}$ and an associated eigenvector $w_{\mathbb{C}} \in D\left(\mathscr{A}_{\mathbb{C}}\right)$ such that $\left\|\operatorname{Re}\left(w_{\mathbb{C}}\right)\right\|_{X}=1$. Assume furthermore that there exist $0 \leq \beta<\gamma_{0}$ and $M_{0}>0$ such that

$$
\left\|\mathrm{e}^{t \mathscr{A}}\right\|_{\mathscr{L}_{C}(X)} \leq M_{0} \mathrm{e}^{\left(\gamma_{0}+\beta\right) t} .
$$

Then, 0 is an unstable solution. More precisely, there exist $K>0, \varepsilon_{0}>0$ and $\delta_{0}>0$ such that, for any $0<\delta<\delta_{0}$, the solution $v$ with initial datum $v^{\text {in }}=\delta \operatorname{Re}\left(w_{\mathbb{C}}\right) \in D(\mathscr{A})$ exists at least on $\left[0, \ln \left(2 \varepsilon_{0} / \delta\right) / \gamma_{0}\right]$ and satisfies, for $0 \leq t \leq \ln \left(2 \varepsilon_{0} / \delta\right) / \gamma_{0}$,

$$
\left\|v(t)-\delta \operatorname{Re}\left(\mathrm{e}^{t \lambda} w_{\mathbb{C}}\right)\right\|_{X} \leq K \delta^{2} \mathrm{e}^{2 t \gamma_{0}} \quad \text { and } \quad\|v(t)\|_{X} \geq \delta \mathrm{e}^{t \gamma_{0}}-K \delta^{2} \mathrm{e}^{2 t \gamma_{0}} .
$$

In particular, for $0<\varepsilon<\varepsilon_{0}$, we see the instability for $t=\left(1 / \gamma_{0}\right) \ln (2 \varepsilon / \delta)$. If $Y$ is a Banach space containing $X$ and with continuous imbedding $X \hookrightarrow Y$, the trivial solution 0 is also unstable from $X$ to $Y$. 
Let us observe that it is always possible to choose the (complex) eigenvector $w$ so that $\operatorname{Re}\left(w_{\mathbb{C}}\right) \neq 0$ since, for any $\theta \in \mathbb{R}, \mathrm{e}^{i \theta} w$ is also an eigenvector. The following corollary deals with the orbital instability. We recall that, under Assumption $A,[\mathscr{L} \mathscr{L}]_{\mathbb{C}}$ has a finite number of eigenvalues in $\{\operatorname{Re}>0\}$.

Corollary B.6. We make Assumption A and suppose that $\mathscr{L} \mathscr{L}$ generates a continuous semigroup. Let 9 be a Banach space containing $\mathscr{X}$ and with continuous imbedding $\mathscr{X} \hookrightarrow$ Y. Assume moreover that $[\mathscr{L} \mathscr{L}]_{\mathbb{C}}$ has at least one eigenvalue in $\{\operatorname{Re}>0\}$ and choose $\lambda \in \mathbb{C}$ with

$$
\operatorname{Re}(\lambda)=\gamma_{0} \equiv \max \left\{\operatorname{Re}(\mu), \mu \in \sigma\left([\mathscr{\mathscr { L }}]_{\mathbb{C}}\right) \cap\{\operatorname{Re}>0\}\right\} \in(0,+\infty)
$$

and $w_{\mathbb{C}} \in D\left(\mathscr{A}_{\mathbb{C}}\right)$ an associated eigenvector such that $\left\|\operatorname{Re}\left(w_{\mathbb{C}}\right)\right\|_{\mathscr{X}}=1$. We assume moreover that

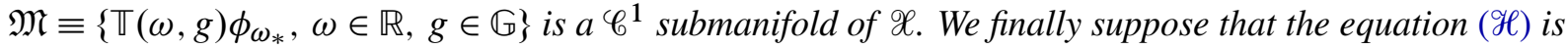
semilinear in the sense that there exists $\Phi: \mathscr{X} \rightarrow \mathscr{X}$ locally Lipschitz continuous such that $\Phi(v)=\mathscr{O}\left(\|v\|_{\mathscr{X}}^{2}\right)$ as $v \rightarrow 0$ and

$$
J\left(E^{\prime}-\omega_{*} Q^{\prime}\right)\left(\phi_{\omega_{*}}+v\right)=J\left(E^{\prime \prime}-\omega_{*} Q^{\prime \prime}\right)\left(\phi_{\omega_{*}}\right)[v]+\Phi(v) .
$$

Then, there exist $K>0, \varepsilon_{0}>0$ and $\delta_{0}>0$, depending only on $\operatorname{Re}\left(w_{\mathbb{C}}\right)$ and $\mathfrak{M}$, with the following properties. For any $0<\delta<\delta_{0}$, the solution $u$ to $(\mathscr{H})$ with initial datum $u^{\text {in }}=\phi_{\omega_{*}}+\delta \operatorname{Re}\left(w_{\mathbb{C}}\right) \in D(\mathscr{A})$ exists at least on $\left[0, \ln \left(2 \varepsilon_{0} / \delta\right) / \gamma_{0}\right]$ and satisfies, for $0 \leq t \leq \ln \left(2 \varepsilon_{0} / \delta\right) / \gamma_{0}$,

$$
\operatorname{dist}_{g}(u(t), \mathfrak{M}) \geq \frac{\delta}{K} \mathrm{e}^{t \gamma_{0}}-K \delta^{2} \mathrm{e}^{2 t \gamma_{0}} .
$$

In particular, the "bound state" solution $T\left(\omega_{*} t\right) \phi_{\omega_{*}}$ is nonlinearly orbitally unstable from $\mathscr{X}$ to $\mathscr{Y}$ and, for $0<\varepsilon<\varepsilon_{0} / K$, we see the instability for $t=\left(1 / \gamma_{0}\right) \ln (2 K \varepsilon / \delta)$.

In [Henry et al. 1982], a similar assertion is made for the orbital instability in the remark after Theorem 2 there, but with $\mathscr{Y}=\mathscr{X}$. For applications to PDEs, the space $\mathscr{X}$ may be a Sobolev space $H^{s}$, and $\mathscr{Y}$ a space like $L^{2}$ or $L^{\infty}$ for instance. The framework of [Grillakis et al. 1987] is the single energy space (for instance $H^{1}$ ), but an instability result established by tracking exponentially growing modes allows proving instability from the regular space $\mathscr{X}\left(H^{1}\right)$ to the nonregular space $\mathscr{y}\left(L^{2}\right.$ or $\left.L^{\infty}\right)$. Here, we may obtain instability in $L^{2}$.

Remark B.7. In the framework of [ibid.], where a Liapounov-type functional is used, it follows that the instability is seen for a time at most equal to $K \varepsilon / \delta^{2}$, where $K$ is some positive constant. This timescale is much larger than the natural one $\left(1 / \gamma_{0}\right) \ln (2 K \varepsilon / \delta)$.

Some applications. We may apply our result to the nonlinear Schrödinger equation

$$
i \partial_{t} \Psi+\Delta \Psi+\Psi f\left(|\Psi|^{2}\right)=0,
$$

or the nonlinear Klein-Gordon equation

$$
\partial_{t}^{2} \Psi=\Delta \Psi+\Psi f\left(|\Psi|^{2}\right)
$$

in $\mathbb{R}^{d}$. We shall consider a nonlinearity $f$ at least $\mathscr{C}^{1}$, so that we are in the framework of [ibid.]. 
- A bound state solution for these two equations is a particular solution of the form $U(t)=e^{i \omega t} \phi_{\omega}$. The instability is in general linked to the fact that

$$
\frac{d}{d \omega} \int_{\mathbb{R}^{d}}\left|\phi_{\omega}\right|^{2} d x<0 \text { for (NLS), } \quad \text { resp. } \quad \frac{d}{d \omega}\left(\omega \int_{\mathbb{R}^{d}}\left|\phi_{\omega}\right|^{2} d x\right)<0 \text { for (NLKG). }
$$

The existence of at least one unstable eigenvalue has been shown under this assumption by [Grillakis 1988] for radial bound states with an arbitrary number of nodes and in [Grillakis et al. 1990] for radial ground states. Corollary B.6 may be applied with $\mathscr{X}=H^{s}\left(\mathbb{R}^{d}\right)$, where $s \in \mathbb{N}, s>d / 2$ and assuming that the nonlinearity satisfies $f \in \mathscr{C}^{s+2}$, and $9=L^{2}\left(\mathbb{R}^{d}\right)$ or $L^{\infty}\left(\mathbb{R}^{d}\right)$. The result in [Mizumachi 2006] shows the instability of linearly unstable bound states for (NLS) (in dimension $d=2$ ) with $f(\varrho)=\varrho^{(p-1) / 2}$ by showing the exponential growth of an unstable eigenmode. Our result gives a simple proof of this result, but restricted to the sufficiently smooth cases, namely $p$ an odd integer or $p>5+2 s>5+d$. For nonsmooth nonlinearities, the situation is more delicate (see [Mizumachi 2006]). An alternative approach is to combine Strichartz estimates with the growth estimate on the semigroup $e^{t \mathscr{L} \mathscr{L}}$ given in Theorem B.4, as in [Georgiev and Ohta 2012].

- Corollary B.6 also applies to the discrete nonlinear Schrödinger equation

$$
i \partial_{t} \Psi_{n}+\varepsilon\left(\Psi_{n+1}-2 \Psi_{n}+\Psi_{n-1}\right)+\Psi_{n} f\left(\left|\Psi_{n}\right|^{2}\right)=0 \text { for all } n \in \mathbb{Z},
$$

as studied in [Melvin et al. 2008] with the saturated nonlinearity $f(\varrho)=\beta /(1+\varrho), \beta>0$ (existence of traveling wave solution) and in [Fitrakis et al. 2007] (defocusing cubic DNLS, i.e., $f(\varrho)=-\beta \varrho$ for some $\beta>0$ ). The numerical analysis in [Fitrakis et al. 2007] shows the existence of linearly unstable bound state solutions. The traveling wave solutions numerically obtained in [Melvin et al. 2008] are linearly stable, but it may happen that, for other nonlinearities $f$, some are linearly unstable.

Quasilinear PDEs. For quasilinear problems, we shall not make restrictions on the smoothness of the nonlinearity. The result relies on the strategy of E. Grenier [2000] and [Rousset and Tzvetkov 2008; 2009]. We consider the evolution equation

$$
\frac{d u}{d t}=J\left(L_{0} u+\nabla F(u)\right)
$$

for $u: \mathbb{R}^{d} \rightarrow \mathbb{R}^{v}$, where $F \in \mathscr{C}^{\infty}\left(\mathbb{R}^{v}, \mathbb{R}\right)$, with the following hypotheses. The operator $J$ is a Fourier multiplier, skew-symmetric on $L^{2}$, into and with domain containing $H^{1}$. There exists $\sigma>0$ such that the operator $L_{0}$ is a Fourier multiplier with domain containing $H^{2 \sigma}$, symmetric and having a self-adjoint realization on $L^{2}\left(\mathbb{R}^{d}, \mathbb{R}^{v}\right)$. Moreover, for some $C>0$, the operator $L_{0}$ satisfies

$$
\frac{1}{C}\|u\|_{H^{\sigma}}^{2} \leq\left(L_{0} u, u\right)_{L^{2}} \leq C\|u\|_{H^{\sigma}}^{2}
$$

The framework proposed in [Rousset and Tzvetkov 2008] was for $L_{0}$ coercive in $H^{1}$; that is, $\sigma=1$. For the examples below, we shall have $\sigma=1 / 2$ or $\sigma=2$, which requires very few modifications to the proof of [ibid.]. We still assume that, for some group $\mathbb{G}$, there exists a unitary representation of $\mathbb{G}$ on $\mathscr{X}$, $\mathbb{T}: \mathbb{G} \rightarrow \mathscr{G}_{c}(\mathscr{X})$, leaving the equation $(\mathrm{E})$ invariant. 
We consider a stationary solution of the evolution equation $(\mathrm{E})$, that is, some $Q \in H^{\infty}\left(\mathbb{R}^{d}, \mathbb{R}^{v}\right)$ such that $L_{0} Q+\nabla F(Q)=0$. We are interested in the stability of this solution. We assume that the commutator $\left[J, \nabla^{2} F(Q)\right]$ is bounded in $L^{2}$, which is the case when $J$ is bounded in $L^{2}$ or when $d=1$ and $J=\partial_{x}$. We suppose that, for the problem

$$
\frac{\partial u}{\partial t}=J\left(L_{0} u+\nabla F\left(u^{a}+u\right)-\nabla F\left(u^{a}\right)+G\right),
$$

where $u^{a}$ is smooth, bounded as well as its derivatives and $G \in \mathscr{C}\left(\mathbb{R}, H^{s}\right)$ for every $s$, we have local well-posedness for $s$ large enough: there exists a time $T>0$ and a unique solution in $\mathscr{C}\left([0, T], H^{s}\right)$. We moreover assume that, for some continuous nondecreasing function $\kappa: \mathbb{R}_{+} \rightarrow \mathbb{R}_{+}$with $\kappa(0)=0$, the tame estimate

$$
\left|\left(\partial_{x}^{\alpha} J\left\{\nabla^{2} F(w+v)[v]\right\}, \partial_{x}^{\alpha} v\right)_{L^{2}}\right| \leq \kappa\left(\|w\|_{W^{s+1, \infty}}+\|v\|_{H^{s}}\right)\|v\|_{H^{s}}^{2},
$$

with $|\alpha| \leq s$, holds true. In order to control high-order derivatives, we finally require that, for $s$ large enough, there exist a self-adjoint operator $\mathbb{M}_{S}$ and $C_{S}$ such that

$$
\left|\left(\mathbb{M}_{s} u, v\right)_{L^{2}}\right| \leq C_{s}\|u\|_{H^{s}}\|v\|_{H^{s}}, \quad\left(\mathbb{M}_{s} u, u\right)_{L^{2}} \geq\|u\|_{H^{s}}^{2}-C_{s}\|u\|_{H^{s-\min (\sigma, 1)}}^{2}
$$

and

$$
\operatorname{Re}\left(J L u, \mathbb{M}_{s} u\right)_{L^{2}} \leq C_{s}\|u\|_{H^{s}}\|u\|_{H^{s-\min (\sigma, 1)}}
$$

(for a criterion which ensures the existence of such a multiplier, see Lemma 5.1 in [ibid.]).

Adapting the strategy of [Rousset and Tzvetkov 2008; 2009], we may deduce the following result. Since the proof is very similar, we omit it.

Theorem B.8. We make the above assumptions and assume moreover that $L_{0}+\nabla^{2} F(Q)$ satisfies Assumption $A$ in $L^{2}$. We assume furthermore that $\left[J\left(L_{0}+\nabla^{2} F(Q)\right)\right]_{\mathbb{C}}$ has an unstable eigenvalue in the right half-plane $\{\operatorname{Re}>0\}$, define

$$
\gamma_{0} \equiv \sup \left\{\operatorname{Re}(\lambda), \lambda \in \sigma\left(\left[J\left(L_{0}+\nabla^{2} F(Q)\right)\right]_{\mathbb{C}}\right) \cap\{\operatorname{Re}>0\}\right\} \in(0,+\infty)
$$

and fix $\lambda \in \sigma\left(\left[J\left(L_{0}+\nabla^{2} F(Q)\right)\right]_{\mathbb{C}}\right)$ satisfying $\operatorname{Re}(\lambda)=\gamma_{0}$ and an associated eigenvector $w_{\mathbb{C}} \in$ $D\left(\left[J\left(L_{0}+\nabla^{2} F(Q)\right)\right]_{\mathbb{C}}\right)$ such that $\left\|\operatorname{Re}\left(w_{\mathbb{C}}\right)\right\|_{H^{s}}=1$. There exists $s_{0} \in \mathbb{N}$ such that, if $s \geq s_{0}, Q$ is nonlinearly unstable from $H^{s}$ to $L^{2}$ and to $L^{\infty}$ : there exist $K>0, \varepsilon_{0}>0$ and $\delta_{0}>0$ such that, for any $0<\delta<\delta_{0}$, the $H^{s}$ solution $u$ to $(\mathrm{E})$ with initial datum $u^{\mathrm{in}}=Q+\delta \operatorname{Re}\left(w_{\mathbb{C}}\right) \in H^{s}$ exists at least on $\left[0, \ln \left(2 \varepsilon_{0} / \delta\right) / \gamma_{0}\right]$ and satisfies, for $0 \leq t \leq \ln \left(2 \varepsilon_{0} / \delta\right) / \gamma_{0}$,

$$
\left\|u(t)-Q-\delta \operatorname{Re}\left(\mathrm{e}^{t \lambda} w_{\mathbb{C}}\right)\right\|_{H^{s}} \leq K \delta^{2} \mathrm{e}^{2 t \gamma_{0}}
$$

hence

$$
\|u(t)-Q\|_{L^{2}} \geq \delta \mathrm{e}^{t \gamma_{0}}-K \delta^{2} \mathrm{e}^{2 t \gamma_{0}} \quad \text { and } \quad\|u(t)-Q\|_{L^{\infty}} \geq \delta \mathrm{e}^{t \gamma_{0}}-K \delta^{2} \mathrm{e}^{2 t \gamma_{0}} .
$$

If , in addition, $\mathfrak{M} \equiv\{\mathbb{T}(g) Q, g \in \mathbb{G}\}$ is a $\mathscr{C}^{1}$ submanifold of $H^{s}$, then we also have

$$
\operatorname{dist}_{L^{2}}(u(t), \mathfrak{M}) \geq K \delta \mathrm{e}^{t \gamma_{0}}-K \delta^{2} \mathrm{e}^{2 t \gamma_{0}} \quad \text { and } \quad \operatorname{dist}_{L} \infty(u(t), \mathfrak{M}) \geq K \delta \mathrm{e}^{t \gamma_{0}}-K \delta^{2} \mathrm{e}^{2 t \gamma_{0}}
$$


In particular, for $0<\varepsilon<\varepsilon_{0} / K$, we see the nonlinear orbital instability for $t=\left(1 / \gamma_{0}\right) \ln (2 K \varepsilon / \delta)$.

Some applications to nonlinear dispersive wave equations. Some model quasilinear equations are given by wave equations (in one space dimension) such as the generalized Korteweg-de Vries equation

$$
\partial_{t} u+\partial_{x}(f(u))+\partial_{x}^{3} u=0
$$

the generalized regularized Korteweg-de Vries equation, also called Benjamin-Bona-Mahony equation or Peregrine equation when $f(u)=u^{2} / 2$,

$$
\partial_{t} u+\partial_{x} u+\partial_{x}(f(u))-\partial_{t} \partial_{x}^{2} u=0,
$$

the generalized regularized Boussinesq equation

$$
\partial_{t}^{2} u-\partial_{x}^{2} u-\partial_{x}^{2}(f(u))-\partial_{t}^{2} \partial_{x}^{2} u=0 .
$$

Each of these equations admits a nontrivial solitary wave solution $u(t, x)=U_{c}(x-c t)$ for $c$ in $(0,+\infty)$, $(1,+\infty)$ and $(-\infty,-1) \cup(1,+\infty)$, respectively. For these solitary wave solutions, the momentum is, respectively,

$$
P\left(U_{c}\right)=\int_{\mathbb{R}} U_{c}^{2} d x=\left\|U_{c}\right\|_{L^{2}}^{2}, \quad P\left(U_{c}\right)=\int_{\mathbb{R}} U_{c}^{2}+\left(\partial_{x} U_{c}\right)^{2} d x, \quad P\left(U_{c}\right)=c \int_{\mathbb{R}} U_{c}^{2}+\left(\partial_{x} U_{c}\right)^{2} d x .
$$

The existence of exactly one unstable eigenvalue has been shown with the use of an Evans function by R. Pego and M. Weinstein [1992] for these three equations under the condition $d P\left(U_{c}\right) / d c<0$. Lopes [2002] also gives a linear instability result. Equations (gBBM) and (grBsq) turn out to be semilinear due to the regularization effect. Indeed, they may be written

$$
\partial_{t} u+\left(1-\partial_{x}^{2}\right)^{-1} \partial_{x} u+\left(1-\partial_{x}^{2}\right)^{-1} \partial_{x}(f(u))=0, \quad \partial_{t}^{2} u-\left(1-\partial_{x}^{2}\right)^{-1} \partial_{x}^{2} u-\left(1-\partial_{x}^{2}\right)^{-1} \partial_{x}^{2}(f(u))=0 .
$$

Therefore, Corollary B.6 applies to these two models and this shows the nonlinear instability when linear instability holds.

In [Lin 2008], some generalizations of the equations (gKdV), (gBBM) and (grBsq) have been proposed that take into account pseudodifferential operators. These are, respectively,

$$
\begin{aligned}
\partial_{t} u+\partial_{x}(f(u))-\partial_{x} \mu u & =0, \\
\partial_{t} u+\partial_{x} u+\partial_{x}(f(u))+\partial_{t} \mu u & =0
\end{aligned}
$$

and

$$
\partial_{t}^{2} u-\partial_{x}^{2} u-\partial_{x}^{2}(f(u))+\partial_{t}^{2} \mu u=0 .
$$

Here, $M$ is a Fourier multiplier of symbol $\hat{M}: \widehat{M w}=\hat{M} \hat{w}$ (here, $\hat{\cdot}$ denotes the Fourier transform). We assume $\hat{M} \geq 0$ (otherwise, see [ibid.]). When $\mathcal{M}=-\partial_{x}^{2}$, these equations reduce to (gKdV), $(\mathrm{gBBM})$ and (grBsq), respectively. The Benjamin-Ono equation $(\hat{\mathcal{M}}=|\xi|)$, the Smith equation $\left(\hat{\mathcal{M}}=\sqrt{1+\xi^{2}}-1\right)$ and the intermediate long wave (or Whitham) equation $(\hat{M}=\xi / \tanh (\xi H)-1 / H$, for some constant $H>0$ ) 
are common models of dispersive wave equations that are of type (I). We refer to [ibid.] for references on these models and the existence of solitary waves. The associated momentum is

$$
P_{\mathrm{I}}\left(U_{c}\right)=\int_{\mathbb{R}} U_{c}^{2} d x=\left\|U_{c}\right\|_{L^{2}}^{2}, \quad P_{\mathrm{II}}\left(U_{c}\right)=\left\|(1+M)^{1 / 2} U_{c}\right\|_{L^{2}}^{2}, \quad P_{\mathrm{III}}\left(U_{c}\right)=c\left\|(1+M)^{1 / 2} U_{c}\right\|_{L^{2}}^{2} .
$$

For these models, Evans function type arguments do not work since we no longer have a differential equation (it is nonlocal). The paper [ibid.] proposes a different approach than the Evans function technique for establishing the existence of unstable eigenvalues. However, it is not completely clear whether this method extends easily to the case of systems such as the Euler-Korteweg system (EK) (given at the beginning of Section $5 \mathrm{~A})$.

Theorem B.9 [Lin 2008]. We consider one of the equations (I), (II) or (III) with $f$ of class $\mathscr{C}^{1}$ satisfying $f(0)=f^{\prime}(0)=0$ and $|f(u)| \gg|u|$ for $|u| \rightarrow+\infty$. We assume moreover that $\hat{M}$ is even, nonnegative, and satisfies, for some $m \geq 1,0<\underline{\lim }_{+\infty} \hat{\mathcal{M}}(\xi) / \xi^{m} \leq \varlimsup_{+\infty} \hat{\mathcal{M}}(\xi) / \xi^{m}<\infty$. Assume that $c \mapsto \phi_{c}=U_{c}(x-c t)$ is a $\mathscr{C}^{1}$ branch of traveling wave solution to (I), (II) or (III) with $U_{c} \in H^{m / 2}(\mathbb{R})$ defined near $c_{*}$ and suppose that the linearized operator $\mathscr{L}$ has exactly one negative eigenvalue, that ker $\mathscr{L}$ is spanned by $\partial_{x} U_{c_{*}}$ and that $\left(d P\left(U_{c}\right) / d c\right)_{\mid c=c_{*}}<0$. Then, $U_{c_{*}}$ is linearly unstable.

It is not easy to determine whether the hypotheses of Theorem B.9 hold true when $M$ is not a (differential) Sturm-Liouville operator. See however [Albert 1992] on this question. It is clear that, if the assumptions of Theorem B.9 are satisfied, then Assumption A is also satisfied. As for the (gBBM) and the (grBsq) equations, the equations (II) and (III) turn out to be semilinear; thus we may prove nonlinear orbital instability by applying Corollary B.6.

The Kawahara equation (or fifth-order KdV equation)

$$
\partial_{t} u+\partial_{x}(f(u))+\alpha \partial_{x}^{3} u+\beta \partial_{x}^{5} u=0
$$

with $\alpha, \beta \neq 0$ two real constants, is another relevant dispersive model. For this equation, it may happen that the linearized equation around the solitary wave has more than one negative eigenvalue, in which case [Grillakis et al. 1987; 1990; Lopes 2002; Lin 2008] do not give a clear necessary and sufficient condition for stability. T. Bridges and G. Derks [2002] give a sufficient condition for linear instability for solitary wave solutions, but also for other types of traveling solutions. This condition is probably not necessary since it may happen that there exist at least two unstable eigenvalues, or two complex conjugate eigenvalues.

Instead of stating a general result for nonlinear orbital instability, we shall consider several model cases on which we will verify the hypotheses of Theorem B.8, in particular the question of the existence of the multiplier $\mathbb{M}_{s}$.

Proposition B.10. We consider the equation (I), namely

$$
\partial_{t} u+\partial_{x}(f(u))-\partial_{x} M u=0,
$$


with $f$ of class $\mathscr{C}^{1}$ satisfying $f(0)=f^{\prime}(0)=0$ and $|f(u)| \gg|u|$ for $|u| \rightarrow+\infty$. We assume that $\hat{\mathcal{M}}$ is one of the following functions:

$$
\begin{aligned}
& -\xi^{2} \quad(K d V) ; \quad \xi^{4}+\alpha \xi^{2} \quad(\text { Kawahara }) ; \quad|\xi| \quad \text { (Benjamin-Ono); } \\
& \frac{\xi}{\tanh (\xi H)}-\frac{1}{H} \quad \text { (intermediate long wave); } \sqrt{1+\xi^{2}}-1 \quad \text { (Smith). }
\end{aligned}
$$

There exists $s_{0}>0$ such that, if there exists $c \in \mathbb{R}$ such that (I) has a nontrivial solitary wave $U_{c} \in L^{2}$ which is linearly unstable, then, for any $s \geq s_{0}$, it is also nonlinearly unstable from $H^{s}$ to $H^{s}$, to $L^{2}$ and to $L^{\infty}$.

By application of Theorem B.8, we are thus able to show the nonlinear instability from $H^{s}$ to $L^{2}$ or $L^{\infty}$ by tracking the exponentially growing mode (this question was left open in [Lin 2008] and also in [Lopes 2002]). In particular, we obtain the $L^{2}$ nonlinear instability of the linearly unstable solitary waves for these models.

Proof. All the assumptions for Theorem B. 8 for these types of models are satisfied in Section 8.1 in [Rousset and Tzvetkov 2008], except the existence of the multiplier $\mathbb{M}_{s}$.

For the $\mathrm{KdV}$ equation, where $\sigma=1$, we shall take (for $s \geq 2$ an integer)

$$
\mathbb{M}_{s} \equiv(-1)^{s} \partial_{x}^{2 s}+\frac{1+2 s}{3}(-1)^{s-1} \partial_{x}^{s-1}\left\{f^{\prime}(Q) \partial_{x}^{s-1} \cdot\right\}
$$

as the computations from [ibid., Section 8.1] show. For the Kawahara equation, with $\sigma=2$, we take (for $s \geq 4$ an integer)

$$
\mathbb{M}_{s} \equiv(-1)^{s} \partial_{x}^{2 s}+\frac{1+2 s}{5}(-1)^{s-1} \partial_{x}^{s-2}\left\{f^{\prime}(Q) \partial_{x}^{s-2} \cdot\right\}
$$

and, since the computations are very similar, we omit them. For the Benjamin-Ono equation, we have $\hat{\mathcal{M}}(\xi)=|\xi|$ and $\sigma=1 / 2$, and we will then have to deal with pseudodifferential operators which are Fourier multipliers with homogeneous symbol. For this type of operator, we shall need some commutator estimates. We denote by $\mathscr{F}(w)$ or $\hat{w}$ the Fourier transform of $w$, and $\mathscr{H}$ the Fourier multiplier with symbol $-i \operatorname{sgn}(\xi)$ (this is the Hilbert transform).

Lemma B.11. (i) Let $h \in L^{\infty}(\mathbb{R})$ with $\mathscr{F}\left(M^{1 / 2} h\right) \in L^{1}(\mathbb{R})$ (for instance, $h \in H^{\sigma}(\mathbb{R})$ for some $\left.\sigma>1\right)$. Then, there exists $C>0$ such that, for any $v \in H^{1 / 2}(\mathbb{R})$,

$$
\left\|M^{\frac{1}{2}}(h v)-h M^{\frac{1}{2}} v\right\|_{L^{2}(\mathbb{R})} \leq C\|v\|_{L^{2}(\mathbb{R})} .
$$

(ii) Let $h \in L^{\infty}(\mathbb{R})$ with $\mathscr{F}\left(M^{3 / 2} h\right) \in L^{1}(\mathbb{R})$ (for instance, $h \in H^{\sigma}(\mathbb{R})$ for some $\left.\sigma>2\right)$. Then, there exists $C>0$ such that, for any $v \in H^{3 / 2}(\mathbb{R})$,

$$
\left\|\mathcal{M}^{\frac{3}{2}}\{h v\}-h \mathcal{M}^{\frac{3}{2}} v-\frac{3}{2}\left[\partial_{x} h\right] \mathcal{M}^{\frac{1}{2}} \mathscr{H} v\right\|_{L^{2}(\mathbb{R})} \leq C\|v\|_{L^{2}(\mathbb{R})} .
$$

(iii) Let $h \in L^{\infty}(\mathbb{R})$ with $\mathscr{F}\left(\partial_{x} M^{1 / 2} h\right) \in L^{1}(\mathbb{R})$ (for instance, $h \in H^{\sigma}(\mathbb{R})$ for some $\left.\sigma>2\right)$. Then, there exists $C>0$ such that, for any $v \in H^{3 / 2}(\mathbb{R})$,

$$
\left\|\partial_{x} M^{\frac{1}{2}}\{h v\}-h \partial_{x} M^{\frac{1}{2}} v-\frac{3}{2}\left[\partial_{x} h\right] M^{\frac{1}{2}} v\right\|_{L^{2}(\mathbb{R})} \leq C\|v\|_{L^{2}(\mathbb{R})} .
$$


Proof. We have

$$
\mathscr{F}\left(M^{\frac{1}{2}}(h v)-h \mu^{\frac{1}{2}} v\right)(\xi)=\int_{\mathbb{R}}|\xi|^{\frac{1}{2}} \hat{h}(\xi-\zeta) \hat{v}(\zeta) d \zeta-\int_{\mathbb{R}}|\zeta|^{\frac{1}{2}} \hat{h}(\xi-\zeta) \hat{v}(\zeta) d \zeta .
$$

Using the inequality $\left.|| \xi\right|^{1 / 2}-|\zeta|^{1 / 2}|\leq C| \xi-\left.\zeta\right|^{1 / 2}$, we thus obtain

$$
\left|\mathscr{F}\left(M^{\frac{1}{2}}(h v)-h \mu^{\frac{1}{2}} v\right)(\xi)\right| \leq C \int_{\mathbb{R}}|\xi-\zeta|^{\frac{1}{2}}|\hat{h}(\xi-\zeta)| \cdot|\hat{v}(\zeta)| d \zeta=C\left\{\left|\mathscr{F}\left(M^{\frac{1}{2}} h\right)\right| *|\hat{v}|\right\}(\xi)
$$

and we conclude with the classical convolution estimate $L^{1} * L^{2} \subset L^{2}$. This argument does not provide the sharpest bound in $h$, since it involves $\left\|\mathscr{F}_{(}\left(M^{1 / 2} h\right)\right\|_{L^{1}}$, whereas the use of paradifferential calculus will use only $\|h\|_{\mathscr{C}_{1 / 2}}$. However, we shall to use this refinement here.

The starting point for the second inequality is

$$
\left.|| \xi\right|^{\frac{3}{2}}-|\zeta|^{\frac{3}{2}}-\frac{3}{2}|\zeta|^{\frac{1}{2}} \operatorname{sgn}(\zeta)(\xi-\zeta)|\leq C| \xi-\left.\zeta\right|^{\frac{3}{2}}
$$

Using the homogeneity $\xi=\theta \zeta$, this is a direct consequence of the easy inequality

$$
\left.|| \theta\right|^{\frac{3}{2}}-1-\frac{3}{2}(\theta-1)|\leq C| \theta-\left.1\right|^{\frac{3}{2}}
$$

Therefore,

$$
\begin{aligned}
\mid \mathscr{F}\left(M^{\frac{3}{2}}\right. & \left.\{h v\}-h \mu^{\frac{3}{2}} v-\frac{3}{2}\left[\partial_{x} h\right] \mu^{\frac{1}{2}} \mathscr{H} v\right)(\xi) \mid \\
& =\left.\left|\int_{\mathbb{R}}\right| \xi\right|^{\frac{3}{2}} \hat{h}(\xi-\zeta) \hat{v}(\zeta) d \zeta-\int_{\mathbb{R}}|\zeta|^{\frac{3}{2}} \hat{h}(\xi-\zeta) \hat{v}(\zeta) d \zeta-\int_{\mathbb{R}} \frac{3}{2}|\zeta|^{\frac{1}{2}} \operatorname{sgn}(\zeta)(\xi-\zeta) \hat{h}(\xi-\zeta) \hat{v}(\zeta) d \zeta \mid \\
& \leq C \int_{\mathbb{R}}|\xi-\zeta|^{\frac{3}{2}}|\hat{h}(\xi-\zeta)| \cdot|\hat{v}(\zeta)| d \zeta \\
& =C\left|\mathscr{F}\left(M^{\frac{3}{2}} h\right)\right| *|\hat{v}|,
\end{aligned}
$$

and we conclude as before. For the third inequality, we argue in a similar way with the estimate

$$
\left.|i \xi| \xi\right|^{\frac{1}{2}}-i \zeta|\zeta|^{\frac{1}{2}}-i \frac{3}{2}|\zeta|^{\frac{1}{2}}(\xi-\zeta)|\leq C| \xi-\left.\zeta\right|^{\frac{3}{2}} .
$$

The proof is complete.

For the Benjamin-Ono equation, $\hat{\mathcal{M}}(\xi)=|\xi|, \sigma=1 / 2$ and the index $s$ will be half an integer: $s \in \mathbb{N} / 2$. Therefore, we set $s=[s]+\{s\}$, with $[s]$ integer and $\{s\} \in\{0 ; 1 / 2\}$. Let us define, for $s \in \mathbb{N} / 2, s \geq 1$,

$$
\mathbb{M}_{s} \equiv \begin{cases}(-1)^{s} \partial_{x}^{2 s}+\gamma_{s} \mathcal{M}^{\frac{1}{2}} \partial_{x}^{s-1}\left\{f^{\prime}(Q) \partial_{x}^{s-1} \mathcal{M}^{\frac{1}{2}} \cdot\right\} & \text { if }\{s\}=0 \\ (-1)^{[s]} \partial_{x}^{2[s]} \mathcal{M}+\gamma_{s} \partial_{x}^{[s]}\left\{f^{\prime}(Q) \partial_{x}^{[s]} \cdot\right\} & \text { if }\{s\}=\frac{1}{2}\end{cases}
$$

for some real constant $\gamma_{s}$ to be determined later. It is clear that $\mathbb{M}_{S}$ is self-adjoint on $L^{2}$ and that there exists $C_{s}>0$ such that

$$
\left|\left(\mathbb{M}_{s} u, v\right)_{L^{2}}\right| \leq C_{s}\|u\|_{H^{s}}\|v\|_{H^{s}} \quad \text { and } \quad\left(\mathbb{M}_{s} u, u\right)_{L^{2}} \geq\|u\|_{H^{s}}^{2}-C_{s}\|u\|_{H^{s-\frac{1}{2}}}^{2}
$$


To verify the assumptions for the multiplier $\mathbb{M}_{s}$, it remains to study $\operatorname{Re}\left(J\left(L_{0}+\nabla^{2} F(Q)\right) u, \mathbb{M}_{s} u\right)_{L^{2}}$. When $\{s\}=0$, i.e., $s \in \mathbb{N}$, this quantity is

$$
\begin{aligned}
& \operatorname{Re}\left(\partial_{x}\left(M+c+f^{\prime}(Q)\right) u, \mathbb{M}_{s} u\right)_{L^{2}} \\
& =\operatorname{Re}\left(\partial_{x} M u,(-1)^{s} \partial_{x}^{2 s} u\right)_{L^{2}}+\gamma_{s} \operatorname{Re}\left(\partial_{x} M u, \mu^{\frac{1}{2}} \partial_{x}^{s-1}\left\{f^{\prime}(Q) \partial_{x}^{s-1} \mu^{\frac{1}{2}} u\right\}\right)_{L^{2}} \\
& \quad+\operatorname{Re}\left(\partial_{x}\left[f^{\prime}(Q) u\right],(-1)^{s} \partial_{x}^{2 s} u\right)_{L^{2}}+\gamma_{s} \operatorname{Re}\left(\partial_{x}\left[f^{\prime}(Q) u\right], \mu^{\frac{1}{2}} \partial_{x}^{s-1}\left\{f^{\prime}(Q) \partial_{x}^{s-1} \mu^{\frac{1}{2}} u\right\}\right)_{L^{2}} \\
& \quad+c \operatorname{Re}\left(\partial_{x} u, \mathbb{M}_{s} u\right)_{L^{2}} .
\end{aligned}
$$

By skew-adjointness, the first and last scalar products are zero. By integration by parts and the Leibniz formula, we deduce, since $Q \in H^{\infty}$,

$$
\begin{aligned}
\operatorname{Re}\left(\partial_{x}\left[f^{\prime}(Q) u\right],\right. & \left.(-1)^{s} \partial_{x}^{2 s} u\right)_{L^{2}} \\
& =\operatorname{Re}\left(\partial_{x}^{s+1}\left[f^{\prime}(Q) u\right], \partial_{x}^{s} u\right)_{L^{2}} \\
& \leq \operatorname{Re}\left(f^{\prime}(Q) \partial_{x}^{s+1} u, \partial_{x}^{s} u\right)_{L^{2}}+(s+1) \operatorname{Re}\left(\partial_{x}\left[f^{\prime}(Q)\right] \partial_{x}^{s} u, \partial_{x}^{s} u\right)_{L^{2}}+C_{s}\|u\|_{H^{s}}\|u\|_{H^{s-1}} \\
& \leq\left(s+\frac{1}{2}\right) \operatorname{Re}\left(\partial_{x}\left[f^{\prime}(Q)\right] \partial_{x}^{s} u, \partial_{x}^{s} u\right)_{L^{2}}+C_{s}\|u\|_{H^{s}}\|u\|_{H^{s-1}} .
\end{aligned}
$$

Similarly, using the easy estimates $\left\|\mathcal{M}^{1 / 2} v\right\|_{L^{2}} \leq K\|v\|_{H^{1 / 2}}$ and $\|h v\|_{H^{1 / 2}} \leq C(h)\|v\|_{H^{1 / 2}}$ for $h \in L^{\infty}$ with $\mathscr{F}\left(\mathcal{M}^{1 / 2} h\right) \in L^{1}$ (this is an immediate consequence of Lemma B.11),

$\gamma_{s} \operatorname{Re}\left(\partial_{x}\left[f^{\prime}(Q) u\right], \mu^{\frac{1}{2}} \partial_{x}^{s-1}\left\{f^{\prime}(Q) \partial_{x}^{s-1} \mu^{\frac{1}{2}} u\right\}\right)_{L^{2}}$

$$
\begin{aligned}
& =\gamma_{s}(-1)^{s-1} \operatorname{Re}\left(M^{\frac{1}{2}} \partial_{x}^{s}\left[f^{\prime}(Q) u\right], f^{\prime}(Q) \partial_{x}^{s-1} \mu^{\frac{1}{2}} u\right)_{L^{2}} \\
& \leq \gamma_{s}(-1)^{s-1} \operatorname{Re}\left(\mathcal{M}^{\frac{1}{2}}\left[f^{\prime}(Q) \partial_{x}^{s} u\right], f^{\prime}(Q) \partial_{x}^{s-1} \mu^{\frac{1}{2}} u\right)_{L^{2}}+C\|u\|_{H^{s-\frac{1}{2}}}^{2}
\end{aligned}
$$

Using Lemma B.11, we deduce $\left\|\mathcal{M}^{1 / 2}\left[f^{\prime}(Q) \partial_{x}^{s} u\right]-f^{\prime}(Q) M^{1 / 2} \partial_{x}^{s} u\right\|_{L^{2}} \leq C(Q)\|u\|_{H^{s}}$; thus $\gamma_{s} \operatorname{Re}\left(\partial_{x}\left[f^{\prime}(Q) u\right], \mu^{\frac{1}{2}} \partial_{x}^{s-1}\left\{f^{\prime}(Q) \partial_{x}^{s-1} \mu^{\frac{1}{2}} u\right\}\right)_{L^{2}}$

$$
\begin{aligned}
& \leq \gamma_{s}(-1)^{s-1} \operatorname{Re}\left(f^{\prime}(Q) \partial_{x}^{s} \mathcal{M}^{\frac{1}{2}} u, f^{\prime}(Q) \partial_{x}^{s-1} \mathcal{M}^{\frac{1}{2}} u\right)_{L^{2}}+C\|u\|_{H^{s}}\|u\|_{H^{s-\frac{1}{2}}} \\
& =\frac{\gamma_{s}}{2}(-1)^{s} \operatorname{Re}\left(\partial_{x}\left[f^{\prime}(Q)\right] \partial_{x}^{s-1} \mathcal{M}^{\frac{1}{2}} u, f^{\prime}(Q) \partial_{x}^{s-1} \mathcal{M}^{\frac{1}{2}} u\right)_{L^{2}}+C\|u\|_{H^{s}}\|u\|_{H^{s-\frac{1}{2}}} \\
& \leq C\|u\|_{H^{s-\frac{1}{2}}}^{2}+C\|u\|_{H^{s}}\|u\|_{H^{s-\frac{1}{2}}} \leq C\|u\|_{H^{s}}\|u\|_{H^{s-\frac{1}{2}}} .
\end{aligned}
$$

We now turn to the term

$$
\gamma_{s} \operatorname{Re}\left(\partial_{x} \mu u, \mu^{\frac{1}{2}} \partial_{x}^{s-1}\left\{f^{\prime}(Q) \partial_{x}^{s-1} \mu^{\frac{1}{2}} u\right\}\right)_{L^{2}}=\gamma_{s}(-1)^{s-1} \operatorname{Re}\left(\partial_{x}^{s} u, \mu^{\frac{3}{2}}\left\{f^{\prime}(Q) \partial_{x}^{s-1} \mu^{\frac{1}{2}} u\right\}\right)_{L^{2}} .
$$

Using Lemma B.11, we write

$$
\begin{aligned}
\left\|\mathcal{M}^{\frac{3}{2}}\left\{f^{\prime}(Q) \partial_{x}^{s-1} \mathcal{M}^{\frac{1}{2}} u\right\}-f^{\prime}(Q) \partial_{x}^{s-1} \mathcal{M}^{2} u-\frac{3}{2} \partial_{x}\left[f^{\prime}(Q)\right] \mathcal{M}^{\frac{1}{2}} \mathscr{H}\left\{\partial_{x}^{s-1} \mathcal{M}^{\frac{1}{2}} u\right\}\right\|_{L^{2}} & \leq C(Q)\left\|\partial_{x}^{s-1} \mathcal{M}^{\frac{1}{2}} u\right\|_{L^{2}} \\
& \leq C(Q)\|u\|_{H^{s-\frac{1}{2}}}
\end{aligned}
$$


which implies

$$
\begin{aligned}
& \gamma_{s} \operatorname{Re}\left(\partial_{x} M u, M^{\frac{1}{2}} \partial_{x}^{s-1}\left\{f^{\prime}(Q) \partial_{x}^{s-1} \mu^{\frac{1}{2}} u\right\}\right)_{L^{2}} \\
& \quad \leq \gamma_{s}(-1)^{s-1} \operatorname{Re}\left(\partial_{x}^{s} u, f^{\prime}(Q) \partial_{x}^{s-1} M^{2} u\right)_{L^{2}}+\frac{3}{2} \gamma_{s}(-1)^{s-1} \operatorname{Re}\left(\partial_{x}^{s} u, \partial_{x}\left[f^{\prime}(Q)\right] \mathcal{M}^{\frac{1}{2}} \mathscr{H}\left\{\partial_{x}^{s-1} \mathcal{M}^{\frac{1}{2}} u\right\}\right)_{L^{2}} \\
& +C\|u\|_{H^{s}}\|u\|_{H^{s-\frac{1}{2}}}
\end{aligned}
$$

Noticing that $\mathcal{M}^{2}=-\partial_{x}^{2}$ and $\mathcal{M}^{1 / 2} \mathscr{H} \partial_{x}^{s-1} \mathcal{M}^{1 / 2}=\partial_{x}^{s-1} \mathcal{M} \mathscr{H}=-\partial_{x}^{s}$ (since $\mathcal{M} \mathscr{H}$ has symbol equal to $-i \xi$ ), we infer

$$
\begin{aligned}
& \gamma_{s} \operatorname{Re}\left(\partial_{x} M u, \mu^{\frac{1}{2}} \partial_{x}^{s-1}\left\{f^{\prime}(Q) \partial_{x}^{s-1} \mathcal{M}^{\frac{1}{2}} u\right\}\right)_{L^{2}} \\
& \quad \leq \gamma_{s}(-1)^{s} \operatorname{Re}\left(\partial_{x}^{s} u, f^{\prime}(Q) \partial_{x}^{s+1} u\right)_{L^{2}}+\frac{3}{2} \gamma_{s}(-1)^{s} \operatorname{Re}\left(\partial_{x}^{s} u, \partial_{x}\left[f^{\prime}(Q)\right] \partial_{x}^{s} u\right)_{L^{2}}+C\|u\|_{H^{s}}\|u\|_{H^{s-\frac{1}{2}}} \\
& \quad=\gamma_{s}(-1)^{s} \operatorname{Re}\left(\partial_{x}^{s} u, \partial_{x}\left[f^{\prime}(Q)\right] \partial_{x}^{s} u\right)_{L^{2}}+C\|u\|_{H^{s}}\|u\|_{H^{s-\frac{1}{2}}}
\end{aligned}
$$

by integration by parts.

Reporting these estimates into (B-1), we infer

$$
\begin{aligned}
& \operatorname{Re}\left(\partial_{x}\left(M+c+f^{\prime}(Q)\right) u, \mathbb{M}_{s} u\right)_{L^{2}} \\
& \quad \leq\left(s+\frac{1}{2}\right) \operatorname{Re}\left(\partial_{x}\left[f^{\prime}(Q)\right] \partial_{x}^{s} u, \partial_{x}^{s} u\right)_{L^{2}}+\gamma_{s}(-1)^{s} \operatorname{Re}\left(\partial_{x}^{s} u, \partial_{x}\left[f^{\prime}(Q)\right] \partial_{x}^{s} u\right)_{L^{2}}+C\|u\|_{H^{s}}\|u\|_{H^{s-\frac{1}{2}}}
\end{aligned}
$$

Therefore, the choice

$$
\gamma_{s} \equiv(-1)^{s-1}\left(s+\frac{1}{2}\right)
$$

provides the desired control

$$
\operatorname{Re}\left(\partial_{x}\left(M+c+f^{\prime}(Q)\right) u, \mathbb{M}_{s} u\right)_{L^{2}} \leq C\|u\|_{H^{s}}\|u\|_{H^{s-\frac{1}{2}}}
$$

When $\{s\}=1 / 2$, the computations are similar: (B-1) becomes now

$$
\begin{aligned}
& \operatorname{Re}\left(\partial_{x}\left(M+c+f^{\prime}(Q)\right) u, \mathbb{M}_{s} u\right)_{L^{2}} \\
& =\operatorname{Re}\left(\partial_{x} M u,(-1)^{[s]} \partial_{x}^{2[s]} \mathcal{M}\right)_{L^{2}}+\gamma_{s} \operatorname{Re}\left(\partial_{x} M u, \partial_{x}^{[s]}\left\{f^{\prime}(Q) \partial_{x}^{[s]} u\right\}\right)_{L^{2}} \\
& \quad+\operatorname{Re}\left(\partial_{x}\left[f^{\prime}(Q) u\right],(-1)^{[s]} \partial_{x}^{2[s]} M u\right)_{L^{2}}+\gamma_{s} \operatorname{Re}\left(\partial_{x}\left[f^{\prime}(Q) u\right], \partial_{x}^{[s]}\left\{f^{\prime}(Q) \partial_{x}^{[s]} u\right\}\right)_{L^{2}} \\
& \quad+c \operatorname{Re}\left(\partial_{x} u, \mathbb{M}_{s} u\right)_{L^{2}},
\end{aligned}
$$

and the first and last scalar products still vanish. Moreover, by integration by parts and the Leibniz formula, we deduce, since $Q \in H^{\infty}$,

$$
\begin{aligned}
\gamma_{s} \operatorname{Re}\left(\partial_{x}\left[f^{\prime}(Q) u\right], \partial_{x}^{[s]}\left\{f^{\prime}(Q) \partial_{x}^{[s]} u\right\}\right)_{L^{2}} & =\gamma_{s}(-1)^{[s]} \operatorname{Re}\left(\partial_{x}^{[s]+1}\left[f^{\prime}(Q) u\right], f^{\prime}(Q) \partial_{x}^{[s]} u\right)_{L^{2}} \\
& \leq \gamma_{s}(-1)^{[s]} \operatorname{Re}\left(f^{\prime}(Q) \partial_{x}^{[s]+1} u, f^{\prime}(Q) \partial_{x}^{[s]} u\right)_{L^{2}}+C\|u\|_{H^{[s]}}^{2} \\
& \leq \gamma_{s}(-1)^{[s]-1} \operatorname{Re}\left(\partial_{x}\left[f^{\prime}(Q)\right] \partial_{x}^{[s]} u, f^{\prime}(Q) \partial_{x}^{[s]} u\right)_{L^{2}}+C\|u\|_{H^{[s]}}^{2} \\
& \leq C\|u\|_{H^{[s]}}^{2}=C\|u\|_{H^{s-\frac{1}{2}}}^{2}
\end{aligned}
$$


Furthermore,

$$
\begin{aligned}
& \operatorname{Re}\left(\partial_{x}\left[f^{\prime}(Q) u\right],(-1)^{[s]} \partial_{x}^{2[s]} M u\right)_{L^{2}} \\
& =\operatorname{Re}\left(M^{\frac{1}{2}} \partial_{x}^{[s]+1}\left[f^{\prime}(Q) u\right], \partial_{x}^{[s]} M^{\frac{1}{2}} u\right)_{L^{2}} \\
& \leq \operatorname{Re}\left(\partial_{x} M^{\frac{1}{2}}\left\{f^{\prime}(Q) \partial_{x}^{[s]} u\right\}, \partial_{x}^{[s]} M^{\frac{1}{2}} u\right)_{L^{2}} \\
& +[s] \operatorname{Re}\left(\partial_{x} M^{\frac{1}{2}}\left\{\partial_{x}\left[f^{\prime}(Q)\right] \partial_{x}^{[s]-1} u\right\}, \partial_{x}^{[s]} M^{\frac{1}{2}} u\right)_{L^{2}}+C\|u\|_{H^{[s]-\frac{1}{2}}}\|u\|_{H^{[s]+\frac{1}{2}}} \cdot
\end{aligned}
$$

For the second scalar product, we write, by Lemma B.11,

$$
\begin{aligned}
\operatorname{Re}\left(\partial _ { x } \mathcal { M } ^ { \frac { 1 } { 2 } } \left\{\partial_{x}\right.\right. & {\left.\left.\left[f^{\prime}(Q)\right] \partial_{x}^{[s]-1} u\right\}, \partial_{x}^{[s]} \mathcal{M}^{\frac{1}{2}} u\right)_{L^{2}} } \\
& =\operatorname{Re}\left(\mathcal{M}^{\frac{1}{2}}\left\{\partial_{x}^{2}\left[f^{\prime}(Q)\right] \partial_{x}^{[s]-1} u\right\}, \partial_{x}^{[s]} \mathcal{M}^{\frac{1}{2}} u\right)_{L^{2}}+\operatorname{Re}\left(\mathcal{M}^{\frac{1}{2}}\left\{\partial_{x}\left[f^{\prime}(Q)\right] \partial_{x}^{[s]} u\right\}, \partial_{x}^{[s]} \mathcal{M}^{\frac{1}{2}} u\right)_{L^{2}} \\
& \leq C\|u\|_{H^{[s]-\frac{1}{2}}}\|u\|_{H^{[s]+\frac{1}{2}}}+\operatorname{Re}\left(\partial_{x}\left[f^{\prime}(Q)\right] \partial_{x}^{[s]} \mathcal{M}^{\frac{1}{2}} u, \partial_{x}^{[s]} \mathcal{M}^{\frac{1}{2}} u\right)_{L^{2}}+C\|u\|_{H}\left[s u \|_{H^{[s]+\frac{1}{2}}}\right. \\
& \leq \operatorname{Re}\left(\partial_{x}\left[f^{\prime}(Q)\right] \partial_{x}^{[s]} \mathcal{M}^{\frac{1}{2}} u, \partial_{x}^{[s]} \mathcal{M}^{\frac{1}{2}} u\right)_{L^{2}}+C\|u\|_{H^{s-\frac{1}{2}}}\|u\|_{H^{s}} .
\end{aligned}
$$

For the first scalar product, we use Lemma B.11 once again:

$$
\begin{aligned}
& \operatorname{Re}\left(\partial_{x} M^{\frac{1}{2}}\left\{f^{\prime}(Q) \partial_{x}^{[s]} u\right\}, \partial_{x}^{[s]} \mu^{\frac{1}{2}} u\right)_{L^{2}} \\
& \quad \leq \operatorname{Re}\left(f^{\prime}(Q) \partial_{x} M^{\frac{1}{2}} \partial_{x}^{[s]} u, \partial_{x}^{[s]} M^{\frac{1}{2}} u\right)_{L^{2}}+\frac{3}{2} \operatorname{Re}\left(\partial_{x}\left[f^{\prime}(Q)\right] \partial_{x}^{[s]} M^{\frac{1}{2}} u, \partial_{x}^{[s]} M^{\frac{1}{2}} u\right)_{L^{2}}+C\|u\|_{H^{s-\frac{1}{2}}}\|u\|_{H^{s}} \\
& \quad \leq \operatorname{Re}\left(\partial_{x}\left[f^{\prime}(Q)\right] \partial_{x}^{[s]} \mu^{\frac{1}{2}} u, \partial_{x}^{[s]} \mathcal{M}^{\frac{1}{2}} u\right)_{L^{2}}+C\|u\|_{H^{s-\frac{1}{2}}}\|u\|_{H^{s}} .
\end{aligned}
$$

As a consequence, since $[s]=s-\frac{1}{2}$,

$\operatorname{Re}\left(\partial_{x}\left[f^{\prime}(Q) u\right],(-1)^{[s]} \partial_{x}^{2[s]} \mu u\right)_{L^{2}} \leq\left(s+\frac{1}{2}\right) \operatorname{Re}\left(\partial_{x}\left[f^{\prime}(Q)\right] \partial_{x}^{[s]} \mu^{\frac{1}{2}} u, \partial_{x}^{[s]} \mu^{\frac{1}{2}} u\right)_{L^{2}}+C\|u\|_{H^{s-\frac{1}{2}}}\|u\|_{H^{s}}$

We turn finally to the term

$$
\gamma_{s} \operatorname{Re}\left(\partial_{x} M u, \partial_{x}^{[s]}\left\{f^{\prime}(Q) \partial_{x}^{[s]} u\right\}\right)_{L^{2}}=\gamma_{s}(-1)^{[s]} \operatorname{Re}\left(\partial_{x}^{[s]} M^{\frac{1}{2}} u, \partial_{x} M^{\frac{1}{2}}\left\{f^{\prime}(Q) \partial_{x}^{[s]} u\right\}\right)_{L^{2}},
$$

and infer, by Lemma B.11,

$$
\begin{aligned}
\gamma_{s} \operatorname{Re}\left(\partial_{x} M u, \partial_{x}^{[s]}\left\{f^{\prime}(Q) \partial_{x}^{[s]} u\right\}\right)_{L^{2}} & \leq \gamma_{s}(-1)^{[s]} \operatorname{Re}\left(\partial_{x}^{[s]} \mathcal{M}^{\frac{1}{2}} u, f^{\prime}(Q) \partial_{x} \mathcal{M}^{\frac{1}{2}} \partial_{x}^{[s]} u\right)_{L^{2}} \\
& +\frac{3}{2} \gamma_{s}(-1)^{[s]} \operatorname{Re}\left(\partial_{x}^{[s]} \mathcal{M}^{\frac{1}{2}} u, \partial_{x}\left[f^{\prime}(Q)\right] \mu^{\frac{1}{2}} \partial_{x}^{[s]} u\right)_{L^{2}}+C\|u\|_{H^{s-\frac{1}{2}}}\|u\|_{H^{s}} \\
& =\gamma_{s}(-1)^{[s]} \operatorname{Re}\left(\partial_{x}^{[s]} \mathcal{M}^{\frac{1}{2}} u, \partial_{x}\left[f^{\prime}(Q)\right] \mu^{\frac{1}{2}} \partial_{x}^{[s]} u\right)_{L^{2}}+C\|u\|_{H^{s-\frac{1}{2}}}\|u\|_{H^{s}}
\end{aligned}
$$

Therefore,

$$
\begin{aligned}
\operatorname{Re}\left(\partial_{x}\left(M+c+f^{\prime}(Q)\right)\right. & \left.u, \mathbb{M}_{s} u\right)_{L^{2}} \\
& \leq\left(s+\frac{1}{2}+\gamma_{s}(-1)^{[s]}\right) \operatorname{Re}\left(\partial_{x}^{[s]} \mathcal{M}^{\frac{1}{2}} u, \partial_{x}\left[f^{\prime}(Q)\right] \mathcal{M}^{\frac{1}{2}} \partial_{x}^{[s]} u\right)_{L^{2}}+C\|u\|_{H^{s-\frac{1}{2}}}\|u\|_{H^{s}}
\end{aligned}
$$

hence choosing $\gamma_{s} \equiv(-1)^{[s]-1}\left(s+\frac{1}{2}\right)$ gives the result. 
It remains to study the cases of the intermediate long wave equation and the Smith equation, for which $\hat{M}$ is, respectively,

$$
\frac{\xi}{\tanh (\xi H)}-\frac{1}{H}, \quad \sqrt{1+\xi^{2}}-1
$$

We denote by $M_{0}$ the operator with symbol $|\xi|$ (the one of the Benjamin-Ono equation), and define $\mathbb{M}_{S}$ as for the Benjamin-Ono case (hence with " $\mathcal{M} "=\mathcal{M}_{0}$ ). We observe that, in both cases, $\tilde{\mathcal{M}} \equiv \mathcal{M}-\mathcal{M}_{0}$ is bounded on $L^{2}$. Indeed, its symbol is continuous in $\mathbb{R}$ and, for $\xi \rightarrow \pm \infty$,

$$
\hat{M}(\xi)=\frac{\xi}{\tanh (\xi H)}-\frac{1}{H}=\frac{\xi}{\operatorname{sgn}(\xi)+O\left(e^{-2|\xi| H}\right)}-\frac{1}{H}=|\xi|-\frac{1}{H}+O\left(|\xi| \mathrm{e}^{-2|\xi| H}\right)
$$

and

$$
\hat{M}(\xi)=\sqrt{1+\xi^{2}}-1=|\xi| \sqrt{1+\xi^{-2}}-1=|\xi|-1+O\left(|\xi|^{-1}\right),
$$

respectively. In the quantity $\operatorname{Re}\left(\partial_{x}\left(\mu+c+f^{\prime}(Q)\right) u, \mathbb{M}_{s} u\right)_{L^{2}}$, we then have to bound from above the extra term $\operatorname{Re}\left(\partial_{x}(\tilde{M} u), \mathbb{M}_{s} u\right)_{L^{2}}$; that is (using the skew-adjointness for the higher-order derivatives in $\mathbb{M}_{S}$ ),

$$
\begin{aligned}
& \operatorname{Re}\left(\partial_{x}(\tilde{M} u), \gamma_{s} \mathcal{M}_{0}^{\frac{1}{2}} \partial_{x}^{s-1}\left\{f^{\prime}(Q) \partial_{x}^{s-1} \mathcal{M}_{0}^{\frac{1}{2}} u\right\}\right)_{L^{2}} \\
& \quad=\gamma_{s}(-1)^{s-1} \operatorname{Re}\left(\partial_{x}^{s} M_{0}^{\frac{1}{2}}(\tilde{M} u), f^{\prime}(Q) \partial_{x}^{s-1} \mu_{0}^{\frac{1}{2}} u\right)_{L^{2}} \quad \text { if }\{s\}=0 \\
& \operatorname{Re}\left(\partial_{x}(\tilde{M} u), \gamma_{s} \partial_{x}^{[s]}\left\{f^{\prime}(Q) \partial_{x}^{[s]} u\right\}\right)_{L^{2}}=\gamma_{s}(-1)^{[s]} \operatorname{Re}\left(\partial_{x}^{[s]+1}(\tilde{M} u), f^{\prime}(Q) \partial_{x}^{[s]} u\right)_{L^{2}} \quad \text { if }\{s\}=\frac{1}{2} .
\end{aligned}
$$

We then note that, in both cases, one may actually split $\tilde{M}=\mathcal{M}-\mathcal{M}_{0}=\tilde{M}_{c}+\tilde{M}_{h}$, where $\tilde{M}_{c}$ is the multiplication by $-1 / H$ (respectively, -1$)$ and $\tilde{M}_{h}$ has a symbol which is continuous in $\mathbb{R}$ and $O\left(|\xi|^{-1}\right.$ ) at infinity, so that $\tilde{\mathcal{M}}_{h}$ is bounded from $H^{\sigma}$ to $H^{\sigma+1}$ if $\sigma \geq 0$. Therefore, when $\{s\}=0$, we easily get

$$
\begin{aligned}
& \operatorname{Re}\left(\partial_{x}(\tilde{M} u), \gamma_{s} M_{0}^{\frac{1}{2}} \partial_{x}^{s-1}\left\{f^{\prime}(Q) \partial_{x}^{s-1} M_{0}^{\frac{1}{2}} u\right\}\right)_{L^{2}} \\
& =\gamma_{s}(-1)^{s-1} \operatorname{Re}\left(\partial_{x}^{s} \mu_{0}^{\frac{1}{2}}\left(\tilde{M}_{c} u\right), f^{\prime}(Q) \partial_{x}^{s-1} \mu_{0}^{\frac{1}{2}} u\right)_{L^{2}}+\gamma_{s}(-1)^{s-1} \operatorname{Re}\left(\partial_{x}^{s} \mu_{0}^{\frac{1}{2}}\left(\tilde{M}_{h} u\right), f^{\prime}(Q) \partial_{x}^{s-1} M_{0}^{\frac{1}{2}} u\right)_{L^{2}} \\
& \leq \frac{1}{2} \gamma_{s}(-1)^{s} \operatorname{Re}\left(\partial_{x}^{s-1} \mu_{0}^{\frac{1}{2}} u, \tilde{M}_{c} \partial_{x}\left[f^{\prime}(Q)\right] \partial_{x}^{s-1} \mu_{0}^{\frac{1}{2}} u\right)_{L^{2}}+C\|u\|_{H^{s-\frac{1}{2}}}^{2} \leq C\|u\|_{H^{s-\frac{1}{2}}}^{2}
\end{aligned}
$$

and similarly when $\{s\}=1 / 2$. Therefore, the estimate

$$
\operatorname{Re}\left(\partial_{x}\left(M+c+f^{\prime}(Q)\right) u, \mathbb{M}_{s} u\right)_{L^{2}} \leq C\|u\|_{H^{s-\frac{1}{2}}}\|u\|_{H^{s}}
$$

remains true for the intermediate long wave equation and the Smith equation. The proof of Proposition B.10 is thus completed by applying Theorem B.9.

We now turn to the deferred proofs of Theorem B.4, Theorem B.5, and Corollary B.6.

Proof of Theorem B.4. We shall prove the resolvent estimate required in Corollary B.3. Let us consider $\lambda=\gamma+i \tau \in \mathbb{C}$ with $\gamma \neq 0$ and the resolvent equation $(\mathscr{L} \mathscr{L}-\lambda) v=\Sigma$, or

$$
(\gamma+i \tau) v=\mathscr{L} \mathscr{L}(v)-\Sigma \text {. }
$$


By hypothesis, the essential spectrum of $\mathscr{L} \mathscr{L}$ is of the form $i\left[\mathbb{R} \backslash\left(-\vartheta_{0},+\vartheta_{0}\right)\right]$. Moreover, we have seen that $\mathscr{L} \mathscr{L}$ has a finite number of eigenvalues in the half-space $\{\operatorname{Re}>0\}$; hence, for $|\tau| \geq \tau_{0}$ sufficiently large, we know that there exists a unique solution $v$ to (B-3). By taking the scalar product with $\mathscr{L}(v)$, we deduce the conservation law

$$
\gamma(v, \mathscr{L}(v))_{\mathscr{L}}=-\operatorname{Re}(\Sigma, \mathscr{L}(v))_{\mathscr{X}} .
$$

By our assumption, there exist a finite (possibly empty) number of eigenvalues in $(-\infty, 0],\left(-\mu_{1}, \ldots,-\mu_{q}\right)$, each one of finite multiplicity. For any $1 \leq k \leq q$, we fix an orthonormal basis $\left(\chi_{k, \ell}\right)_{1 \leq \ell \leq n_{k}}$ of the eigenspace $\operatorname{ker}\left(\mathscr{L}+\mu_{k}\right)$. By Assumption A, any eigenvector $\chi_{k, \ell}$ is smooth in the sense that $\chi_{k, \ell} \in D(\mathscr{F})$ and $\mathscr{E} \chi_{k, \ell} \in D(\mathscr{L})$.

We then make a spectral orthogonal decomposition

$$
v=\sum_{k=1}^{q} \sum_{\ell=1}^{n_{k}} \alpha_{k, \ell} \chi_{k, \ell}+v_{+},
$$

where $\mathscr{L}\left(\chi_{k, \ell}\right)=\mu_{k} \chi_{k, \ell}$ and $\left(v_{+}, \mathscr{L}\left(v_{+}\right)\right)_{\mathscr{L}} \geq \delta\left\|v_{+}\right\|_{\mathscr{C}}^{2}$ for some positive $\delta$. In the double sum, we have a finite number (independent of $v$ ) of terms. Inserting this into (B-4) yields $|\gamma| \delta\left\|v_{+}\right\|_{\mathscr{X}}^{2} \leq|\gamma| \delta\left(v_{+}, \mathscr{L}\left(v_{+}\right)\right)_{\mathscr{X}} \leq \delta\left[\left|\operatorname{Re}(\Sigma, \mathscr{L}(v))_{\mathscr{X}}\right|+\sum_{k, \ell} \mu_{k}\left|\alpha_{k, \ell}\right|^{2}\right] \leq K\|\Sigma\|_{\mathscr{X}}\|v\|_{\mathscr{C}}+K \sum_{k, \ell}\left|\alpha_{k, \ell}\right|^{2}$.

Using the inequality $a b \leq \varepsilon a^{2}+b^{2} /(4 \varepsilon)$ with $a=\|v\|_{\mathscr{X}}, b=K\|\Sigma\|_{\mathscr{P}}$ and $\varepsilon=|\gamma| \delta / 2$, the equality $\|v\|_{\mathscr{L}}^{2}=\left\|v_{+}\right\|_{\mathscr{L}}^{2}+\sum_{k, \ell}\left|\alpha_{k, \ell}\right|^{2}$ and incorporating the term $|\gamma| \delta\left\|v_{+}\right\|_{\mathscr{L}}^{2} / 2$ in the left-hand side, we infer

$$
\frac{|\gamma| \delta}{2}\left\|v_{+}\right\|_{\mathscr{C}}^{2} \leq K^{\prime} \sum_{k, \ell}\left|\alpha_{k, \ell}\right|^{2}+K^{\prime \prime}\|\Sigma\|_{\mathscr{Q}}^{2} .
$$

On the other hand, since $\chi_{k, \ell} \in D(\mathscr{F})$ and $\mathscr{\Psi} \chi_{k, \ell} \in D(\mathscr{L})$ by Assumption A, taking the scalar product of (B-1) with $\chi_{k, \ell}$ provides

$$
(\gamma+i \tau) \alpha_{k, \ell}=-\left(v, \mathscr{L} \mathscr{\mathscr { L }} \chi_{k, \ell}\right) \mathscr{x}-\left(\Sigma, \chi_{k, \ell}\right) \mathscr{x} .
$$

Consequently,

$$
(|\gamma|+|\tau|)\left|\alpha_{k, \ell}\right| \leq K_{k, \ell}\|v\|_{\mathscr{L}}+K\|\Sigma\|_{\mathscr{L}}
$$

thus

$$
(|\gamma|+|\tau|)^{2} \sum_{k, \ell}\left|\alpha_{k, \ell}\right|^{2} \leq K_{0}\|v\|_{\mathscr{C}}^{2}+K\|\Sigma\|_{\mathscr{P}}^{2}=K_{0} \sum_{k, \ell}\left|\alpha_{k, \ell}\right|^{2}+K\left\|v_{+}\right\|_{\mathscr{C}}^{2}+K\|\Sigma\|_{\mathscr{X}}^{2}
$$

which implies, if $|\tau| \geq 1+\sqrt{K_{0}}-|\gamma|$,

$$
\sum_{k, \ell}\left|\alpha_{k, \ell}\right|^{2} \leq K \frac{\left\|v_{+}\right\|_{\mathscr{L}}^{2}+\|\Sigma\|_{\mathscr{L}}^{2}}{(|\gamma|+|\tau|)^{2}-K_{0}} .
$$


Reporting this into (B-5) gives

$$
\frac{|\gamma| \delta}{2}\left\|v_{+}\right\|_{\mathscr{C}}^{2} \leq K^{\prime} K \frac{\left\|v_{+}\right\|_{\mathscr{P}}^{2}+\|\Sigma\|_{\mathscr{P}}^{2}}{(|\gamma|+|\tau|)^{2}-K_{0}}+K^{\prime \prime}\|\Sigma\|_{\mathscr{C}}^{2} .
$$

If $|\tau| \geq 1+\sqrt{K_{0}+4 K K^{\prime} /|\gamma| \delta}-|\gamma|$, we deduce

$$
\frac{|\gamma| \delta}{4}\left\|v_{+}\right\|_{\mathscr{Q}}^{2} \leq\left(K^{\prime \prime}+\frac{K^{\prime} K}{(|\gamma|+|\tau|)^{2}-K_{0}}\right)\|\Sigma\|_{\mathscr{C}}^{2} \leq K_{1}\|\Sigma\|_{\mathscr{C}}^{2}
$$

and it follows that

$$
\|v\|_{\mathscr{Q}}^{2}=\left\|v_{+}\right\|_{\mathscr{Q}}^{2}+\sum_{k, \ell}\left|\alpha_{k, \ell}\right|^{2} \leq K_{2}\|\Sigma\|_{\mathscr{X}}^{2}
$$

where $K_{2}$ does not depend on $|\tau|$ (large enough), as wished.

The proof of the first semigroup estimate then follows easily; see, for instance, Proposition 2 in [Prüss 1984].

Proof of the semigroup estimate when $\gamma_{0}>\mathbf{0}$. Here, we assume $\gamma_{0}>0$. As a consequence, the spectrum of $[\mathscr{\mathscr { L }}]_{\mathbb{C}}$ is of the form $\sigma_{\mathrm{s}} \cup \sigma_{\mathrm{u}}$, where $\sigma_{\mathrm{ess}}\left([\mathscr{\mathscr { L }}]_{\mathbb{C}}\right) \subset \sigma_{\mathrm{s}} \subset\{\operatorname{Re} \leq 0\}$ and $\varnothing \neq \sigma_{\mathrm{u}} \subset\{\operatorname{Re}>0\}$ consists in a finite number of eigenvalues of finite algebraic multiplicities. Therefore, we may define (see, e.g., [Kato 1976; Hislop and Sigal 1996]) the spectral Riesz projection

$$
\mathbb{P} \equiv \frac{1}{2 i \pi} \int_{\Gamma}\left([\mathscr{L}]_{\mathbb{C}}-z\right)^{-1} d z
$$

where $\Gamma$ is any simple (positively oriented) closed curve enclosing $\sigma_{\mathrm{u}}$. As a consequence, $\mathbb{P}$ is bounded, commutes with $[\mathscr{\mathscr { L }}]_{\mathbb{C}}$ on $D\left([\mathscr{L}]_{\mathbb{C}}\right)$ and satisfies $\sigma\left([\mathscr{\mathscr { L }}]_{\mathbb{C}} \mathbb{P}\right)=\sigma_{\mathrm{u}}, \sigma\left([\mathscr{\mathscr { L }}]_{\mathbb{C}}(\mathrm{Id}-\mathbb{P})\right)=\sigma_{\mathrm{s}}$. Moreover, $[\mathscr{L}]_{\mathbb{C}} \mathbb{P}$ is bounded, and hence generates a continuous semigroup, $e^{t[\mathscr{\mathscr { L }}]_{\mathbb{C}} \mathbb{P}}$, given by the exponential series

$$
\mathrm{e}^{t[\mathscr{\mathscr { L }}]_{\mathbb{C}} \mathbb{P}}=\sum_{n=0}^{+\infty} \frac{t^{n}\left([\mathscr{\mathscr { L }}]_{\mathbb{C}} \mathbb{P}\right)^{n}}{n !} .
$$

In addition, $[\mathscr{F} \mathscr{L}]_{\mathbb{C}}(\mathrm{Id}-\mathbb{P})=[\mathscr{E} \mathscr{L}]_{\mathbb{C}}-[\mathscr{\mathscr { L }}]_{\mathbb{C}} \mathbb{P}$ also generates a continuous semigroup and we have $e^{t[\mathscr{F} L]_{\mathbb{C}}}=\mathrm{e}^{t \mathscr{L} \mathscr{P} \mathbb{P}} \mathrm{e}^{t[\mathscr{Y} \mathscr{L}]_{\mathbb{C}}(\mathrm{Id}-\mathbb{P})}$.

The semigroup generated by the bounded operator $[\mathscr{F} \mathscr{L}]_{\mathbb{C}} \mathbb{P}$ is easily analyzed. We shall now apply the spectral mapping theorem of J. Prüss (Theorem B.2) to $[\mathscr{F} \mathscr{L}]_{\mathbb{C}}(\mathrm{Id}-\mathbb{P})$ in order to control the growth of its norm. By Corollary B.3, it suffices to estimate its resolvent $\left[[\mathscr{\mathscr { L }}]_{\mathbb{C}}(\mathrm{Id}-\mathbb{P})-(\gamma+i \tau)\right]^{-1}$ for large $|\tau|$ (note that $\left.\sigma\left([\mathscr{\mathscr { L }}]_{\mathbb{C}}(\mathrm{Id}-\mathbb{P})\right)=\sigma_{\mathrm{s}} \subset\{\operatorname{Re} \leq 0\}\right)$. If $\Sigma \in \mathscr{X}_{\mathbb{C}}$ and $|\tau|$ is large, it is clear that the solution $u \in \mathscr{X}_{\mathbb{C}}$ to $\left[[\mathscr{\mathscr { L }}]_{\mathbb{C}}(\mathrm{Id}-\mathbb{P})-(\gamma+i \tau)\right] u=\Sigma$ is given by

$$
u=\left[[\mathscr{L} \mathscr{L}]_{\mathbb{C}}-(\gamma+i \tau)\right]^{-1}(\mathrm{Id}-\mathbb{P}) \Sigma-\frac{1}{\gamma+i \tau} \mathbb{P} \Sigma
$$

thus, for $|\tau|$ large,

$$
\left\|\left[[\mathscr{\mathscr { L }}]_{\mathbb{C}}(\mathrm{Id}-\mathbb{P})-(\gamma+i \tau)\right]^{-1}\right\|_{\mathscr{L}_{C}(\mathscr{L})} \leq\left\|\left[[\mathscr{\mathscr { L }}]_{\mathbb{C}}-(\gamma+i \tau)\right]^{-1}\right\|_{\mathscr{L}_{C}(\mathscr{L} C)}\|\mathrm{Id}-\mathbb{P}\|_{\mathscr{L}_{C}(\mathscr{X})}+\frac{1}{|\gamma+i \tau|}\|\mathbb{P}\|_{\mathscr{L}_{\mathcal{C}}(\mathscr{L})}
$$


is bounded. Consequently, by Theorem B.2 and since $\sigma_{\mathrm{s}} \subset\{\operatorname{Re} \leq 0\}, \sigma\left(\mathrm{e}^{t[\mathscr{F} \mathscr{L}]_{\subset}(\mathrm{Id}-\mathbb{P})}\right)=\mathrm{e}^{t \sigma\left([\mathscr{\mathscr { L }}]_{\mathbb{C}}(\mathrm{Id}-\mathbb{P})\right)}=$ $\mathrm{e}^{t \sigma_{\mathrm{s}}} \subset \bar{D}(0,1)$. It follows that, for any $\epsilon>0$, there exists $K_{\epsilon}>0$ such that

$$
\left\|\mathrm{e}^{t[\mathscr{F}]_{\mathbb{C}}(\mathrm{Id}-\mathbb{P})}\right\|_{\mathscr{L}_{\mathcal{C}}(\mathscr{L} C)} \leq K_{\epsilon} \mathrm{e}^{\epsilon t} \text { for all } t \geq 0 .
$$

Since $e^{t[\mathscr{L}]_{\mathbb{C}} \mathbb{P}}$ is given by the exponential series, we also have the optimal estimate

$$
\left\|\mathrm{e}^{t[\mathscr{\mathscr { L }}]_{\mathbb{C}} \mathbb{P}}\right\|_{\mathscr{L}_{C}(\mathscr{L} \mathbb{C})} \leq K_{0}(1+t)^{m-1} \mathrm{e}^{\gamma_{0} t} \text { for all } t \geq 0
$$

by definition of $m$. We conclude by taking $\epsilon=\gamma_{0} / 2$ for instance.

Proof of Theorem B.5. Since $\mathscr{A}$ generates a continuous semigroup, $v$ is a solution to $\partial_{t} v=\mathscr{A} v+\Phi(v)$ if and only if it is a mild solution:

$$
v(t)=\mathrm{e}^{t \mathscr{A}} v^{\mathrm{in}}+\int_{0}^{t} \mathrm{e}^{(t-\tau) \mathscr{A}} \Phi(v(\tau)) d \tau .
$$

There exists $r_{0}>0$ such that $\|\Phi(v)\|_{X} \leq M\|v\|_{X}^{2}$ if $\|v\|_{X} \leq r_{0}$. We choose $v^{\text {in }}=\delta \operatorname{Re} w$, where $\|\operatorname{Re} w\|_{X}=1$ and $w$ is an eigenvector for the eigenvalue $\lambda$, and write the solution under the form $v=\mathrm{e}^{t \not} v^{\text {in }}+\tilde{v}=\operatorname{Re}\left(\mathrm{e}^{t \lambda} w\right)+\tilde{v}$. If $\lambda \in \mathbb{R}$, we can choose $w \in D(\mathscr{A}) \subset D\left(\mathscr{A}_{\mathbb{C}}\right)$. Then,

$$
\tilde{v}(t)=\int_{0}^{t} \mathrm{e}^{(t-\tau) \mathscr{A}} \Phi\left(\delta \operatorname{Re}\left(\mathrm{e}^{t \lambda} w\right)+\tilde{v}(\tau)\right) d \tau .
$$

Let us define $r_{1} \equiv \min \left(r_{0},\left(\gamma_{0}-\beta\right) /\left(2 M M_{0}\right)\right)$ and let $T>0$ be the maximal time such that $T<$ $\ln (r /(2 \delta)) / \gamma_{0}$ and $\|\tilde{u}(\tau)\|_{X}<r_{1} / 2$ in $[0, T)$, where $0<r<r_{1}$ will be determined later. We shall work for $0 \leq t<T$, so that $\left\|\delta \operatorname{Re}\left(\mathrm{e}^{t \lambda} w\right)+\tilde{v}(\tau)\right\|_{X}<\delta \mathrm{e}^{t \gamma_{0}}+r_{1} / 2 \leq r_{1} \leq r_{0}$. Then,

$$
\begin{aligned}
\|\tilde{v}(t)\| & \leq \int_{0}^{t}\left\|\mathrm{e}^{(t-\tau) \mathscr{A}}\right\|_{\mathscr{L}_{C}(X)} M\left\|\delta \operatorname{Re}\left(\mathrm{e}^{\tau \lambda} w\right)+\tilde{v}(\tau)\right\|^{2} d \tau \\
& \leq 2 M_{0} M \int_{0}^{t} \mathrm{e}^{\left(\gamma_{0}+\beta\right)(t-\tau)}\left(\delta^{2} \mathrm{e}^{2 \tau \gamma_{0}}+\|\tilde{v}(\tau)\|^{2}\right) d \tau \\
& \leq \frac{2 M_{0} M}{\gamma_{0}-\beta} \delta^{2} \mathrm{e}^{2 t \gamma_{0}}+r_{1} M_{0} M \int_{0}^{t} \mathrm{e}^{\left(\gamma_{0}+\beta\right)(t-\tau)}\|\tilde{v}(\tau)\|_{X} d \tau,
\end{aligned}
$$

since $\beta<\gamma_{0}$. Applying now the Gronwall inequality to $\mathrm{e}^{-\left(\gamma_{0}+\beta\right) t}\|\tilde{v}(t)\|_{X}$ then gives, since $M_{0} M r_{1}<$ $\gamma_{0}-\beta$,

$$
\|\tilde{u}(t)\|_{X} \leq\left[\frac{2 M_{0} M}{\gamma_{0}-\beta}+\frac{2 r_{1} M_{0}^{2} M^{2}}{\left(\gamma_{0}-\beta\right)\left(\gamma_{0}-\beta-r_{1} M_{0} M\right)}\right] \delta^{2} \mathrm{e}^{2 t \gamma_{0}}=K \delta^{2} \mathrm{e}^{2 t \gamma_{0}} .
$$

We now choose $r \equiv \sqrt{r_{1} / K}$, so that the right-hand side is $\leq K r^{2} / 4<r_{1} / 2$, and this implies that $u$ exists at least on $\left[0, \ln (r /(2 \delta)) / \gamma_{0}\right]$. In addition, for $0 \leq t<T$,

$$
\|u(t)\|_{X} \geq \delta \mathrm{e}^{t \gamma_{0}}-\|\tilde{u}(t)\|_{X} \geq \delta \mathrm{e}^{t \gamma_{0}}-K \delta^{2} \mathrm{e}^{2 t \gamma_{0}},
$$

as desired. We conclude choosing $\varepsilon_{0}>0$ so small that $2 \varepsilon_{0}-K \varepsilon_{0}^{2} \geq \varepsilon_{0}$. 
Proof of Corollary B.6. We pick some $0<\beta<\gamma_{0}$ (for instance $\beta=\gamma_{0} / 2$ ) in order to have the semigroup estimate required in Theorem B.5. The solution $u(t)=T\left(\omega_{*} t\right)\left(\phi_{\omega_{*}}+v(t)\right)$ satisfies, for $0 \leq t \leq$ $\gamma_{0}^{-1} \ln \left(2 \varepsilon_{0} / \delta\right)$

$$
\|v(t)\|_{\mathscr{X}}=\left\|T\left(-\omega_{*} t\right) u(t)-\left(\phi_{\omega_{*}}+\delta \operatorname{Re}\left(\mathrm{e}^{t \lambda} w\right)\right)\right\|_{\mathscr{X}} \leq K \delta^{2} \mathrm{e}^{2 t \gamma_{0}} .
$$

Hence, $T\left(-\omega_{*} t\right) u(t)$ remains at distance $\leq K \varepsilon_{0}$ from $\phi_{\omega_{*}} \in \mathfrak{M}$ and therefore

$$
\operatorname{dist} \mathscr{X}(u(t), \mathfrak{M}) \geq \operatorname{dist} \mathscr{L}\left(\delta \operatorname{Re}\left(\mathrm{e}^{t \lambda} w\right), \mathfrak{M}-\phi\right)-K \delta^{2} \mathrm{e}^{2 t \gamma_{0}} .
$$

Assume $\lambda \in \mathbb{R}$. Then, we observe that the straight line $\mathbb{R} \ni \theta \mapsto \theta w$ is transverse to the tangent space $\mathrm{T}_{\phi} \mathfrak{M}$ of the manifold $\mathfrak{M}$, since $w$ is an eigenvector of $\mathscr{L} \mathscr{L}$ for $\lambda \neq 0$, and hence does not belong to the kernel of $\mathscr{L}$. Therefore, $\operatorname{dist} \mathscr{x}(\theta w, \mathfrak{M}-\phi) \geq|\theta| / K_{1}$ for small $|\theta|$. Thus,

$$
\operatorname{dist}_{\mathscr{X}}(u(t), \mathfrak{M}) \geq \frac{1}{K_{1}} \delta \mathrm{e}^{t \lambda}-K \delta^{2} \mathrm{e}^{2 t \gamma_{0}} .
$$

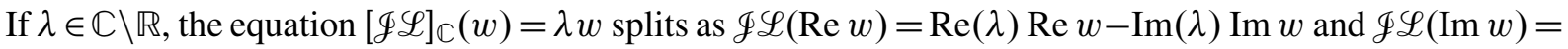
$\operatorname{Im}(\lambda) \operatorname{Re} w+\operatorname{Re}(\lambda) \operatorname{Im} w$. Therefore, $\operatorname{Re} w$ and $\operatorname{Im} w$ do not belong to $\operatorname{ker}(\mathscr{L})$. Consequently, the surface $\mathbb{C} \ni \theta \mapsto \operatorname{Re}(\theta w)$ is transverse to the tangent space $\mathrm{T}_{\phi} \mathfrak{M}$ of the manifold $\mathfrak{M}$, and we conclude as before that

$$
\operatorname{dist}_{\mathscr{L}}(u(t), \mathfrak{M}) \geq \frac{1}{K_{1}} \delta \mathrm{e}^{t \gamma_{0}}-K \delta^{2} \mathrm{e}^{2 t \gamma_{0}}
$$

\section{Acknowledgement}

We acknowledge the support of the French ANR (Agence Nationale de la Recherche) under Grant ANR JC ArDyPitEq.

\section{References}

[Abid et al. 2003] M. Abid, C. Huepe, S. Metens, C. Nore, C. T. Pham, L. S. Tuckerman, and M. E. Brachet, "GrossPitaevskii dynamics of Bose-Einstein condensates and superfluid turbulence”, Fluid Dynam. Res. 33:5-6 (2003), 509-544. MR 2004k:82115 Zbl 1060.76528

[Albert 1992] J. P. Albert, "Positivity properties and stability of solitary-wave solutions of model equations for long waves", Comm. Partial Differential Equations 17:1-2 (1992), 1-22. MR 93d:35140 Zbl 0782.35064

[Barashenkov 1996] I. V. Barashenkov, "Stability criterion for dark solitons”, Phys. Rev. Lett. 77:7 (1996), 1193-1197.

[Barashenkov and Panova 1993] I. V. Barashenkov and E. Panova, "Stability and evolution of the quiescent and travelling solitonic bubbles", Physica D 69:1-2 (1993), 114-134. Zbl 0791.35126

[Benzoni-Gavage 2010a] S. Benzoni-Gavage, "Propagating phase boundaries and capillary fluids", Lecture notes, Centro Internazionale per la Ricerca Matematica, 2010, Available at http://www.science.unitn.it/cirm/Benzoni-course.pdf.

[Benzoni-Gavage 2010b] S. Benzoni-Gavage, "Spectral transverse instability of solitary waves in Korteweg fluids", J. Math. Anal. Appl. 361:2 (2010), 338-357. MR 2011f:35267 Zbl 1180.35451

[Benzoni-Gavage et al. 2005] S. Benzoni-Gavage, R. Danchin, S. Descombes, and D. Jamet, "Structure of Korteweg models and stability of diffuse interfaces", Interfaces Free Bound. 7:4 (2005), 371-414. MR 2006h:35227 Zbl 1106.35056

[Berestycki and Cazenave 1981] H. Berestycki and T. Cazenave, "Instabilité des états stationnaires dans les équations de Schrödinger et de Klein-Gordon non linéaires”, C. R. Acad. Sci. Paris Sér. I Math. 293:9 (1981), 489-492. MR 84f:35120 Zbl 0492.35010 
[Béthuel et al. 2008a] F. Béthuel, P. Gravejat, and J.-C. Saut, "Existence and properties of travelling waves for the GrossPitaevskii equation", pp. 55-103 in Stationary and time dependent Gross-Pitaevskii equations (Vienna, 2006), edited by A. Farina and J.-C. Saut, Contemp. Math. 473, Amer. Math. Soc., Providence, RI, 2008. MR 2011a:35484 Zbl 1216.35132

[Béthuel et al. 2008b] F. Béthuel, P. Gravejat, J.-C. Saut, and D. Smets, "Orbital stability of the black soliton for the GrossPitaevskii equation”, Indiana Univ. Math. J. 57:6 (2008), 2611-2642. MR 2010d:35333 Zbl 1171.35012

[Béthuel et al. 2010] F. Béthuel, R. Danchin, and D. Smets, "On the linear wave regime of the Gross-Pitaevskii equation", $J$. Anal. Math. 110 (2010), 297-338. MR 2012c:35409 Zbl 1202.35296

[Bogdan et al. 1989] M. Bogdan, A. Kovalev, and A. Kosevich, "Stability criterion in imperfect Bose gas", Fiz. Nizk. Temp. 15:5 (1989), 511-513. In Russian.

[Bona et al. 1987] J. L. Bona, P. E. Souganidis, and W. A. Strauss, "Stability and instability of solitary waves of Korteweg-de Vries type", Proc. Roy. Soc. London Ser. A 411:1841 (1987), 395-412. MR 88m:35128 Zbl 0648.76005

[de Bouard 1995] A. de Bouard, "Instability of stationary bubbles", SIAM J. Math. Anal. 26:3 (1995), 566-582. MR 96i:35120 Zbl 0823.35017

[Bridges and Derks 2002] T. J. Bridges and G. Derks, "Linear instability of solitary wave solutions of the Kawahara equation and its generalizations", SIAM J. Math. Anal. 33:6 (2002), 1356-1378. MR 2003h:35220 Zbl 1011.35117

[Cazenave and Lions 1982] T. Cazenave and P.-L. Lions, "Orbital stability of standing waves for some nonlinear Schrödinger equations", Comm. Math. Phys. 85:4 (1982), 549-561. MR 84i:81015 Zbl 0513.35007

[Chiron 2012] D. Chiron, "Travelling waves for the nonlinear Schrödinger equation with general nonlinearity in dimension one", Nonlinearity 25:3 (2012), 813-850. MR 2887994 Zbl 06030687

[Chiron and Scheid 2012] D. Chiron and C. Scheid, "Travelling waves for the nonlinear Schrödinger equation with general nonlinearity in dimension two", preprint, 2012, Available at http://math.unice.fr/ chiron/fichierspdf/Endim2.pdf. Zbl 06030687

[Comech and Pelinovsky 2003] A. Comech and D. Pelinovsky, "Purely nonlinear instability of standing waves with minimal energy”, Comm. Pure Appl. Math. 56:11 (2003), 1565-1607. MR 2005h:37176 Zbl 1072.35165

[Comech et al. 2007] A. Comech, S. Cuccagna, and D. E. Pelinovsky, "Nonlinear instability of a critical traveling wave in the generalized Korteweg-de Vries equation”, SIAM J. Math. Anal. 39:1 (2007), 1-33. MR 2008f:35336 Zbl 1136.35077

[Di Menza and Gallo 2007] L. Di Menza and C. Gallo, "The black solitons of one-dimensional NLS equations", Nonlinearity 20:2 (2007), 461-496. MR 2007k:35455 Zbl 1128.35095

[Fakau and Karval'u 2009] M. Fakau and M. I. Karval'u, "Stability of dark screening solitons in photorefractive media", Teoret. Mat. Fiz. 160:1 (2009), 49-58. In Russian; translated in Theoret. and Math. Phys. 160:1 (2009), 917-924. MR 2011c:35534 Zbl 1179.78093

[Fitrakis et al. 2007] E. P. Fitrakis, P. G. Kevrekidis, H. Susanto, and D. J. Frantzeskakis, "Dark solitons in discrete lattices: saturable versus cubic nonlinearities", Phys. Rev. E (3) 75:6 (2007), 066608, 12. MR 2008i:37174

[Gallo 2004] C. Gallo, "Schrödinger group on Zhidkov spaces", Adv. Differential Equations 9:5-6 (2004), 509-538. MR 2006d. 35255 Zbl 1103.35093

[Gallo 2008] C. Gallo, "The Cauchy problem for defocusing nonlinear Schrödinger equations with non-vanishing initial data at infinity”, Comm. Partial Differential Equations 33:4-6 (2008), 729-771. MR 2009c:35438 Zbl 1156.35086

[Gardner and Zumbrun 1998] R. A. Gardner and K. Zumbrun, "The gap lemma and geometric criteria for instability of viscous shock profiles”, Comm. Pure Appl. Math. 51:7 (1998), 797-855. MR 99c:35152 Zbl 0933.35136

[Gearhart 1978] L. Gearhart, "Spectral theory for contraction semigroups on Hilbert space", Trans. Amer. Math. Soc. 236 (1978), 385-394. MR 57 \#1191 Zbl 0326.47038

[Georgiev and Ohta 2012] V. Georgiev and M. Ohta, "Nonlinear instability of linearly unstable standing waves for nonlinear Schrödinger equations”, J. Math. Soc. Japan 64:2 (2012), 533-548. MR 2916078 Zbl 1253.35158

[Gérard 2008] P. Gérard, "The Gross-Pitaevskii equation in the energy space", pp. 129-148 in Stationary and time dependent Gross-Pitaevskii equations (Vienna, 2006), edited by A. Farina and J.-C. Saut, Contemp. Math. 473, Amer. Math. Soc., Providence, RI, 2008. MR 2010e:35259 Zbl 1166.35373

[Gérard and Zhang 2009] P. Gérard and Z. Zhang, "Orbital stability of traveling waves for the one-dimensional Gross-Pitaevskii equation”, J. Math. Pures Appl. (9) 91:2 (2009), 178-210. MR 2011f:35322 Zbl 1232.35152 
[Gesztesy et al. 2000] F. Gesztesy, C. K. R. T. Jones, Y. Latushkin, and M. Stanislavova, "A spectral mapping theorem and invariant manifolds for nonlinear Schrödinger equations”, Indiana Univ. Math. J. 49:1 (2000), 221-243. MR 2001g:37144 Zbl 0969.35123

[Ginzburg and Pitaevskii 1958] V. L. Ginzburg and L. P. Pitaevskiĭ, "On the theory of superfluidity", Soviet Physics. JETP 7:5 (1958), 858-861. MR 21 \#4663

[Grenier 2000] E. Grenier, "On the nonlinear instability of Euler and Prandtl equations", Comm. Pure Appl. Math. 53:9 (2000), 1067-1091. MR 2001i:76056 Zbl 1048.35081

[Grillakis 1988] M. Grillakis, "Linearized instability for nonlinear Schrödinger and Klein-Gordon equations", Comm. Pure Appl. Math. 41:6 (1988), 747-774. MR 89m:35192 Zbl 0632.70015

[Grillakis et al. 1987] M. Grillakis, J. Shatah, and W. Strauss, "Stability theory of solitary waves in the presence of symmetry, I", J. Funct. Anal. 74:1 (1987), 160-197. MR 88g:35169 Zbl 0656.35122

[Grillakis et al. 1990] M. Grillakis, J. Shatah, and W. Strauss, "Stability theory of solitary waves in the presence of symmetry, II", J. Funct. Anal. 94:2 (1990), 308-348. MR 92a:35135 Zbl 0711.58013

[Gross 1963] E. P. Gross, "Hydrodynamics of a superfluid condensate", J. Math. Phys. 4:2 (1963), 195-207.

[Henry et al. 1982] D. B. Henry, J. F. Perez, and W. F. Wreszinski, "Stability theory for solitary-wave solutions of scalar field equations", Comm. Math. Phys. 85:3 (1982), 351-361. MR 83m:35131 Zbl 0546.35062

[Hislop and Sigal 1996] P. D. Hislop and I. M. Sigal, Introduction to spectral theory, with applications to Schrödinger operators, Applied Mathematical Sciences 113, Springer, New York, 1996. MR 98h:47003 Zbl 0855.47002

[Kapitula and Sandstede 1998] T. Kapitula and B. Sandstede, "Stability of bright solitary-wave solutions to perturbed nonlinear Schrödinger equations”, Phys. D 124:1-3 (1998), 58-103. MR 99h:35199 Zbl 0935.35150

[Kato 1976] T. Kato, Perturbation theory for linear operators, 2nd ed., Grundlehren der Mathematischen Wissenschaften 132, Springer, Berlin, 1976. MR 53 \#11389 Zbl 0342.47009

[Kivshar and Krolikowski 1995] Y. S. Kivshar and W. Krolikowski, "Instabilities of dark solitons", Optics Letters 20:14 (1995), $1527-1529$.

[Kivshar and Luther-Davies 1998] Y. S. Kivshar and B. Luther-Davies, "Dark optical solitons: physics and applications", Physics Reports 298:2-3 (1998), 81-197.

[Kivshar and Yang 1994] Y. S. Kivshar and X. Yang, "Perturbation-induced dynamics of dark solitons", Phys. Rev. E (3) 49:2 (1994), 1657-1670. MR 96k:78007

[Kolomeisky et al. 2000] E. B. Kolomeisky, T. J. Newman, J. P. Straley, and X. Qi, "Low-dimensional Bose liquids: beyond the Gross-Pitaevskii approximation”, Phys. Rev. Lett. 85:8 (2000), 1146-1149.

[Lin 2002] Z. Lin, "Stability and instability of traveling solitonic bubbles", Adv. Differential Equations 7:8 (2002), 897-918. MR 2003d:35243 Zbl 1033.35117

[Lin 2008] Z. Lin, "Instability of nonlinear dispersive solitary waves", J. Funct. Anal. 255:5 (2008), 1191-1224. MR 2010m. 35456 Zbl 1157.35096

[Lopes 2002] O. Lopes, “A linearized instability result for solitary waves”, Discrete Contin. Dyn. Syst. 8:1 (2002), 115-119. MR 2002j:35264 Zbl 1001.35108

[Maeda 2012] M. Maeda, "Stability of bound states of Hamiltonian PDEs in the degenerate cases", J. Funct. Anal. 263:2 (2012), 511-528. MR 2923422 Zbl 1244.35008

[Melvin et al. 2008] T. R. O. Melvin, A. R. Champneys, P. G. Kevrekidis, and J. Cuevas, "Travelling solitary waves in the discrete Schrödinger equation with saturable nonlinearity: existence, stability and dynamics", Phys. D 237:4 (2008), 551-567. MR 2009h:35405 Zbl 1167.35448

[Mizumachi 2006] T. Mizumachi, "A remark on linearly unstable standing wave solutions to NLS”, Nonlinear Anal. 64:4 (2006), 657-676. MR 2007f:35273 Zbl 1091.35093

[Ohta 2011] M. Ohta, "Instability of bound states for abstract nonlinear Schrödinger equations", J. Funct. Anal. 261:1 (2011), 90-110. MR 2012g:37134 Zbl 1228.34092

[Papanicolaou and Spathis 1999] N. Papanicolaou and P. N. Spathis, "Semitopological solitons in planar ferromagnets", Nonlinearity 12:2 (1999), 285-302. MR 99k:82076 Zbl 0938.35183 
[Pego and Weinstein 1992] R. L. Pego and M. I. Weinstein, "Eigenvalues, and instabilities of solitary waves", Philos. Trans. Roy. Soc. London Ser. A 340:1656 (1992), 47-94. MR 93g:35115 Zbl 0776.35065

[Pelinovsky and Kevrekidis 2008] D. E. Pelinovsky and P. G. Kevrekidis, "Dark solitons in external potentials", Z. Angew. Math. Phys. 59:4 (2008), 559-599. MR 2009i:35306 Zbl 1157.35089

[Prüss 1984] J. Prüss, "On the spectrum of $C_{0}$-semigroups", Trans. Amer. Math. Soc. 284:2 (1984), 847-857. MR 85f:47044 Zbl 0572.47030

[Roberts and Berloff 2001] P. Roberts and N. Berloff, "The nonlinear Schrödinger equation as a model of superfluid helium", pp. 235-257 in Quantized vortex dynamics and superfluid turbulence, edited by C. F. Barenghi et al., Lecture Notes in Physics 571, Springer, Berlin, 2001. Zbl 0994.82105

[Rousset and Tzvetkov 2008] F. Rousset and N. Tzvetkov, "Transverse nonlinear instability of solitary waves for some Hamiltonian PDE's”, J. Math. Pures Appl. (9) 90:6 (2008), 550-590. MR 2011a:35473 Zbl 1159.35063

[Rousset and Tzvetkov 2009] F. Rousset and N. Tzvetkov, "Transverse nonlinear instability for two-dimensional dispersive models", Ann. Inst. H. Poincaré Anal. Non Linéaire 26:2 (2009), 477-496. MR 2010j:35475 Zbl 1169.35374

[Sandstede 2002] B. Sandstede, "Stability of travelling waves", pp. 983-1055 in Handbook of dynamical systems, II, edited by B. Fiedler, North-Holland, Amsterdam, 2002. MR 2004e:37121 Zbl 1056.35022

[Shatah and Strauss 1985] J. Shatah and W. Strauss, "Instability of nonlinear bound states", Comm. Math. Phys. 100:2 (1985), 173-190. MR 87b:35159 Zbl 0603.35007

[Shatah and Strauss 2000] J. Shatah and W. Strauss, "Spectral condition for instability", pp. 189-198 in Nonlinear PDE's, dynamics and continuum physics (South Hadley, MA, 1998), edited by J. Bona et al., Contemp. Math. 255, Amer. Math. Soc., Providence, RI, 2000. MR 2001c:34127 Zbl 0960.47033

[Souganidis and Strauss 1990] P. E. Souganidis and W. A. Strauss, "Instability of a class of dispersive solitary waves", Proc. Roy. Soc. Edinburgh Sect. A 114:3-4 (1990), 195-212. MR 92a:35143 Zbl 0713.35108

[Tsuzuki 1971] T. Tsuzuki, "Nonlinear waves in the Pitaevskii-Gross equation", J. Low Temp. Phys. 4:4 (1971), 441-457.

[Weinstein 1986] M. I. Weinstein, "Lyapunov stability of ground states of nonlinear dispersive evolution equations", Comm. Pure Appl. Math. 39:1 (1986), 51-67. MR 87f:35023 Zbl 0594.35005

[Zhidkov 2001] P. E. Zhidkov, Korteweg-de Vries and nonlinear Schrödinger equations: qualitative theory, Lecture Notes in Mathematics 1756, Springer, Berlin, 2001. MR 2002h:35285 Zbl 0987.35001

[Zumbrun 2008] K. Zumbrun, "A sharp stability criterion for soliton-type propagating phase boundaries in Korteweg's model”, Z. Anal. Anwend. 27:1 (2008), 11-30. MR 2009h:35391 Zbl 1187.35229

Received 25 Jun 2012. Revised 4 Sep 2012. Accepted 28 Feb 2013.

DAVID CHIRON: chiron@unice.fr

Laboratoire J.A. Dieudonné, Université de Nice-Sophia Antipolis, Parc Valrose, 06108 Nice Cedex 02, France 


\title{
Analysis \& PDE
}

\author{
msp.org/apde
}

\section{EDITORS}

EDITOR-IN-CHIEF

\author{
Maciej Zworski \\ zworski@math.berkeley.edu \\ University of California \\ Berkeley, USA
}

BOARD OF EDITORS

Nicolas Burq Université Paris-Sud 11, France

nicolas.burq@math.u-psud.fr

Sun-Yung Alice Chang Princeton University, USA

chang@math.princeton.edu

Michael Christ University of California, Berkeley, USA

mchrist@math.berkeley.edu

Charles Fefferman Princeton University, USA

cf@math.princeton.edu

Ursula Hamenstaedt Universität Bonn, Germany

ursula@math.uni-bonn.de

Vaughan Jones U.C. Berkeley \& Vanderbilt University vaughan.f.jones@vanderbilt.edu

Herbert Koch Universität Bonn, Germany koch@math.uni-bonn.de

Izabella Laba University of British Columbia, Canada ilaba@math.ubc.ca

Gilles Lebeau Université de Nice Sophia Antipolis, France lebeau@unice.fr

László Lempert Purdue University, USA lempert@math.purdue.edu

Richard B. Melrose Massachussets Institute of Technology, USA rbm@math.mit.edu

Frank Merle Université de Cergy-Pontoise, France Frank.Merle@u-cergy.fr

William Minicozzi II Johns Hopkins University, USA minicozz@math.jhu.edu

Werner Müller Universität Bonn, Germany mueller@math.uni-bonn.de
Yuval Peres

Gilles Pisier

Tristan Rivière

Igor Rodnianski

Wilhelm Schlag

Sylvia Serfaty

Yum-Tong Siu

Terence Tao

Michael E. Taylor

Gunther Uhlmann

András Vasy

Dan Virgil Voiculescu

Steven Zelditch
University of California, Berkeley, USA

peres@stat.berkeley.edu

Texas A\&M University, and Paris 6

pisier@math.tamu.edu

ETH, Switzerland

riviere@math.ethz.ch

Princeton University, USA

irod@math.princeton.edu

University of Chicago, USA

schlag@math.uchicago.edu

New York University, USA

serfaty@cims.nyu.edu

Harvard University, USA

siu@math.harvard.edu

University of California, Los Angeles, USA

tao@math.ucla.edu

Univ. of North Carolina, Chapel Hill, USA

met@math.unc.edu

University of Washington, USA

gunther@math.washington.edu

Stanford University, USA

andras@math.stanford.edu

University of California, Berkeley, USA

dvv@math.berkeley.edu

Northwestern University, USA

zelditch@math.northwestern.edu

\section{PRODUCTION}

production@msp.org

Silvio Levy, Scientific Editor

See inside back cover or msp.org/apde for submission instructions.

The subscription price for 2013 is US \$160/year for the electronic version, and \$310/year ( $\$ 35$, if shipping outside the US) for print and electronic. Subscriptions, requests for back issues from the last three years and changes of subscribers address should be sent to MSP.

Analysis \& PDE (ISSN 1948-206X electronic, 2157-5045 printed) at Mathematical Sciences Publishers, 798 Evans Hall \#3840, c/o University of California, Berkeley, CA 94720-3840, is published continuously online. Periodical rate postage paid at Berkeley, CA 94704, and additional mailing offices.

APDE peer review and production are managed by EditFLOW ${ }^{\circledR}$ from Mathematical Sciences Publishers.

PUBLISHED BY

- mathematical sciences publishers

nonprofit scientific publishing

http://msp.org/

(C) 2013 Mathematical Sciences Publishers 


\section{ANALYSIS \& PDE Volume $6 \quad$ No. $6 \quad 2013$}

A Nekhoroshev-type theorem for the nonlinear Schrödinger equation on the torus

ERWAN FAOU and BENOÎT GRÉBERT

$L^{q}$ bounds on restrictions of spectral clusters to submanifolds for low regularity metrics MATTHEW D. BLAIR

From the Laplacian with variable magnetic field to the electric Laplacian in the semiclassical 1289 limit

NICOLAS RAYMOND

Stability and instability for subsonic traveling waves of the nonlinear Schrödinger equation in 1327 dimension one

DAVID CHIRON

Semiclassical measures for inhomogeneous Schrödinger equations on tori

NiCOLAS BURQ

Decay of viscous surface waves without surface tension in horizontally infinite domains

YAN GUO and IAN TICE 